\title{
Struktur und Dynamik kleinskaliger Magnetfelder der Sonnenatmosphäre
}

Ergebnisse hochaufgelöster Polarimetrie und Bildrekonstruktion

\author{
Dissertation \\ zur Erlangung des Doktorgrades \\ der Mathematisch-Naturwissenschaftlichen Fakultäten \\ der Georg-August-Universität zu Göttingen
}

\author{
vorgelegt von \\ KATJA JANSSEN \\ aus \\ Bremerhaven
}

Göttingen, 2003 
D7

Referent: Prof. Dr. F. Kneer

Koreferent: Prof. Dr. K. Beuermann

Tag der mündlichen Prüfung: 2. Juli 2003 
Es ist besser ein einziges kleines Licht anzuzünden, als die Dunkelheit zu verfluchen. Konfuzius 



\section{Inhaltsverzeichnis}

\begin{tabular}{lll}
\hline & Einleitung & 9
\end{tabular}

1.1 Aktivität der Sonne . . . . . . . . . . . . . . . . . . . . . . . . . 10

1.2 Kleinskalige Magnetfelder $\ldots \ldots \ldots \ldots \ldots \ldots \ldots$. . . . . . . . . . . . . . 12

$1.2 .1 \quad$ Entstehung und Lebensdauer . . . . . . . . . . . . . . . . . . . . . 12

1.2 .2 Kontrast und Temperatur . . . . . . . . . . . . . . . . . . . . . . . . . . . . . 13

$1.2 .3 \quad$ Magnetfeld und Inklination . . . . . . . . . . . . . . . . . . . . . 14

1.2 .4 Geschwindigkeiten . . . . . . . . . . . . . . . . . . . . . . . . . . . . . . . . . 15

1.2 .5 Heizung höherer Schichten . . . . . . . . . . . . . . . . . . . . . . . . . . . . . . 16

1.2 .6 Schwingungen und Wellen $\ldots \ldots \ldots \ldots$. . . . . . . . . . . . . . . . . . . . .

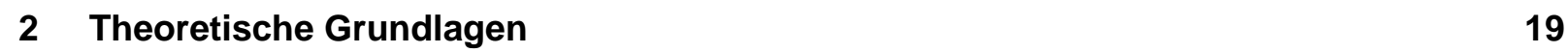

2.1 Zeeman-Effekt . . . . . . . . . . . . . . . . . . . . . . . . . . . . . . . 19

2.1 .1 Aufspaltung der Zeeman-Komponenten . . . . . . . . . . . . . . . . 19

2.1 .2 Polarisation der Zeeman-Komponenten . . . . . . . . . . . . . . 21

2.2 Dopplerbreite von Spektrallinien . . . . . . . . . . . . . . . . . . 22

2.3 Der Stokes-Vektor . . . . . . . . . . . . . . . . . . . . . . . . . . . 23

2.4 Strahlungstransport im Magnetfeld $\ldots \ldots \ldots \ldots$. . . . . . . . . . . . . . 24

$\begin{array}{lll}3 & \text { Beobachtungen } & 27\end{array}$

3.1 Vakuum-Turm-Teleskop . . . . . . . . . . . . . . . . . . 27

3.2 Göttinger Spektropolarimeter . . . . . . . . . . . . . . . . . . . . . . . . 28

$3.2 .1 \quad$ Fabry-Perot-Interferometer . . . . . . . . . . . . . . . . . . . 29

3.2 .2 Stokes- $V$-Polarimeter . . . . . . . . . . . . . . . . . . 31

3.2 .3 Strahlengang . . . . . . . . . . . . . . . . . 32

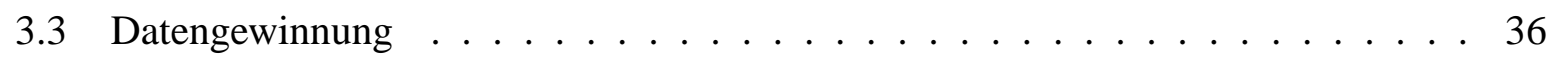

3.3 .1 Beobachtungspraxis . . . . . . . . . . . . . 36

3.3 .2 Großjustage . . . . . . . . . . . . . . . . . . . . . . 39

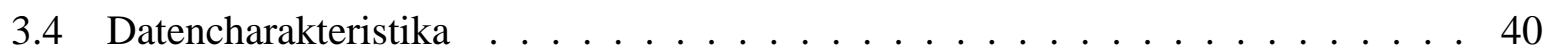

3.4.1 Organisation der Aufnahmen . . . . . . . . . . . . . . . . . . 41

3.4 .2 Wellenlängenverschiebung . . . . . . . . . . . . . . . . . 43 


\section{Inhaltsverzeichnis}

4 Datenreduktion $\quad 45$

4.1 Speckle-Rekonstruktion . . . . . . . . . . . . . . . . . . . . . . . 46

4.2 Rekonstruktion der Schmalbandbilder . . . . . . . . . . . . . . . . . . . . 48

4.3 Neuer CCD2-Programmcode . . . . . . . . . . . . . . . . . . . . . . . 50

4.3 .1 Vorbereitung der Daten . . . . . . . . . . . . . . . 51

4.3 .2 Flatfield und Dark . . . . . . . . . . . . . . . . . . . 52

4.3 .3 Tracking . . . . . . . . . . . . . . . . . . 53

4.3 .4 Rekonstruktion . . . . . . . . . . . . . . . . . . . 53

4.3 .5 Optimumfilter . . . . . . . . . . . . . . . . . . 54

$4.3 .6 \quad$ Wellenlängenverschiebung . . . . . . . . . . . . . . . . 55

$4.3 .7 \quad$ Unverbreitertes Flatfieldprofil $\ldots \ldots \ldots$. . . . . . . . . . . . . . . . . . . . . . . . . . . . 55

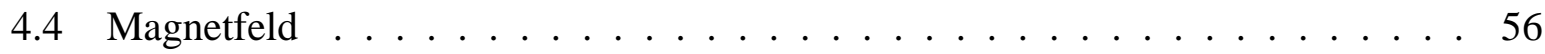

$4.4 .1 \quad$ Crosstalk $\ldots \ldots \ldots \ldots \ldots \ldots \ldots$

4.4 .2 Flatfieldkorrektur zweiter Ordnung . . . . . . . . . . . . . . 58

$4.4 .3 \quad$ Magnetfeld aus der Stokes- $V$-Amplitude . . . . . . . . . . . . . . . . 59

4.4.4 Magnetfeld aus Linienschwerpunkten . . . . . . . . . . . . . . . . . . 60

$4.4 .5 \quad$ Korrektur der Intensitätsabhängigkeit . . . . . . . . . . . . . . . . . . . . 61

4.5 Geschwindigkeit . . . . . . . . . . . . . . . . . 62

4.5 .1 Geschwindigkeit aus dem Stokes- $I$-Schwerpunkt . . . . . . . . . . . 62

4.5 .2 Geschwindigkeit aus dem Stokes- $V$-Nulldurchgang . . . . . . . . . . 63

$\begin{array}{lll}5 & \text { Ergebnisse } & 65\end{array}$

$5.1 \quad$ Die Hausdorff-Dimension magnetischer Strukturen . . . . . . . . . . . . . . . 65

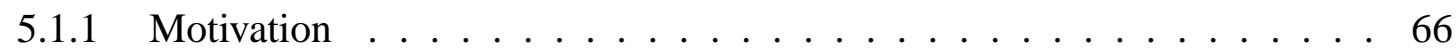

5.1 .2 Mathematische Definition . . . . . . . . . . . . . 67

5.1 .3 Reale Strukturen . . . . . . . . . . . . . . . . . . . . . 68

5.1 .4 Praktische Umsetzung . . . . . . . . . . . . . . . . . . . 69

5.1 .5 Mitte-Rand-Variation . . . . . . . . . . . . . . . . . . 71

5.1 .6 Beobachtete Magnetfelder . . . . . . . . . . . . . . . . . . . . 73

5.1 .7 Simulierte Magnetfelder . . . . . . . . . . . . . . . . . . . . . . . 74

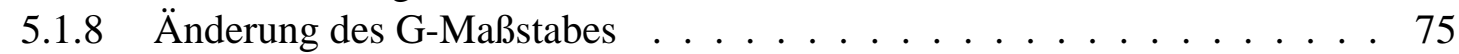

5.1 .9 Auflösungsreduktion der Simulation . . . . . . . . . . . . . . . . . 78

5.1 .10 Schwellwert der Magnetfeldstärke . . . . . . . . . . . . . . . . . . 80

5.1 .11 Rauschen . . . . . . . . . . . . . . . . . . . . 84

5.1 .12 Seeingparameter . . . . . . . . . . . . . . . . 85

5.1 .13 Behandlung des Bildrandes . . . . . . . . . . . . . . . . . . . 88

5.1 .14 Große Strukturen . . . . . . . . . . . . . . . . . . . . . . . . . . . 89

5.1 .15 Kreise $\ldots \ldots \ldots \ldots \ldots$. . . . . . . . . . . . . . . . . . . . . . . . . . . . . .

5.1 .16 Zusammenfassung . . . . . . . . . . . . . . . . . . 91

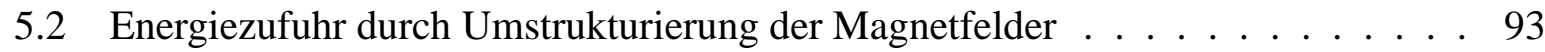

5.2 .1 Motivation . . . . . . . . . . . . . . . . . . 93

5.2 .2 Änderung der Querschnittsfläche . . . . . . . . . . . . . . . . . . . . . . . . . . . . . . . . . . 94

5.2 .3 Horizontale Geschwindigkeiten . . . . . . . . . . . . . . . . . . . . 95 
Inhaltsverzeichnis

$5.2 .4 \quad$ Energiedichte . . . . . . . . . . . . . . . . . . . . . . . . . . . . . 97

5.2 .5 Magnetischer Energiestrom . . . . . . . . . . . . . . . . . . . 99

5.3 Magnetfelder und Zero-Crossing-Geschwindigkeiten $\ldots \ldots \ldots$. . . . . . 100

$5.3 .1 \quad$ Vergleich der Magnetfeldkarten $\ldots \ldots \ldots$. . . . . . . . . . 100

5.3 .2 Zero-Crossing Geschwindigkeit . . . . . . . . . . . . . . . . 102

5.4 Morphologie und Dynamik: "Bright Points" und strukturierte Gebiete . . . . . 105

6 Zusammenfassung und Ausblick 115

$\begin{array}{ll}\text { Literaturverzeichnis } & 119\end{array}$ 
Inhaltsverzeichnis 


\section{Einleitung}

Schon immer war die Sonne aus Sicht der Menschheit da, und doch ist sie so wenig nur erforscht. Erst 1543 erkannte Kopernikus, dass die Sonne das Zentrum unseres Planetensystems ist. Mit Hilfe eines Fernrohres sah Galileo Galilei 1610, dass es sich bei den dunklen Punkten auf der Sonnenscheibe nicht um vorbeiziehende Planeten, sondern um Sonnenflecken handelt und Christoph Scheiner folgerte aus der Bewegung der Sonnenflecken, dass die Sonne rotiert. Er bestimmte ihre Rotationsperiode zu 27 Tagen.

Die mittlere Entfernung der Erde von der Sonne, Astronomische Einheit genannt, konnte erstmals 1769 von Edmund Halley durch gleichzeitige Parallaxenmessungen der Sonne von verschiedenen Punkten der Erde abgeschätzt werden. Erst durch diese Entfernungsbestimmungen bekam der Mensch eine Vorstellung von den Weiten des Weltalls. Aus modernen, auf RadarEchos von der Venus basierenden Messungen und den Kepler'schen Gesetzen berechnet sich die Astronomische Einheit zu $A E=149,6 \cdot 10^{6} \mathrm{~km}$. Da die Erde eine leicht elliptische Bahn um die Sonne beschreibt, variiert die Entfernung um 2,5 $\cdot 10^{6} \mathrm{~km}$, die Astronomische Einheit beschreibt daher einen Mittelwert. Die Entfernungsbestimmung liefert den Schlüssel zu vielen anderen Fragen der Astronomie. So lässt sich z.B. über ihre Entfernung und das dritte Kepler'sche Gesetz die Masse der Sonne bestimmen, sie beträgt $M_{\odot}=1,989 \cdot 10^{30} \mathrm{~kg}$. Ebenfalls aus der Entfernung der Sonne und ihrem Winkeldurchmesser von 1919,26" ergibt sich ihr Radius zu $R_{\odot}=6,960 \cdot 10^{8} \mathrm{~m}$.

Eine wichtige Größe zur Beschreibung eines Sternes ist seine Leuchtkraft $L$, die Gesamtenergie, die er pro Zeiteinheit ausstrahlt. Die Leuchtkraft der Sonne beträgt $L=3,844 \cdot 10^{26} \mathrm{~W}$. Für das Leben auf der Erde jedoch ist vielleicht eine andere Größe, die direkt mit der Leuchtkraft zusammenhängt, von noch größerem Interesse: Die Energie, die die Sonne täglich in Form von Strahlung an die Erde abgibt. Diese für eine mittlere Entfernung der Erde bestimmte Strahlung beträgt $S=1367 \mathrm{~W} / \mathrm{m}^{2}$ und wird Solarkonstante genannt. Sowohl die Leuchtkraft $L$ als auch die Strahlung $S$ werden durch Integralbildung über das gesamte elektromagnetische Spektrum bestimmt. Das Spektrum, die Zerlegung des Lichtes in seine einzelnen Wellenlängenanteile, ist die wichtigste Informationsquelle der Astrophysik.

Schon 1806 wurden von Wollaston dunkle Absorptionslinien in dem spektral aufgespaltenen Licht der Sonne entdeckt, nicht jedoch der Sonne zugeordnet. Josepf Fraunhofer war es, der 1814 die Absorptionlinien als Eigenschaft der Sonne erkannte. 45 Jahre später wurden sie von Gustav Kirchhoff und Robert Bunsen als "Fingerabdrücke" der Elemente in der Sonnenatmosphäre gedeutet. Die Spektrallinien geben durch ihre Breite und Verschiebung Auskunft über Geschwindigkeit und Temperatur der Elemente an ihrem Entstehungsort auf der Sonne, die Tiefe einer Linie besagt etwas über die Häufigkeit des verursachenden Elementes und die Aufspaltung einer Linie in mehrere Teillinien deutet auf ein Magnetfeld hin, das eine solche Aufspaltung be- 


\section{Einleitung}

wirkt. Die Untersuchung dieser magnetischen Strukturen in der Photosphäre der Sonne wird das Thema dieser Arbeit sein.

\subsection{Aktivität der Sonne}

Die Sonne zeigt eine Vielzahl an Aktivität: Sonnenflecken, Plages, Protuberanzen, Ausbrüche der Korona, aber auch viel kleinere Strukturen wie einzelne Poren oder Fackelpunkte. All diese Phänomene besitzen die gleiche Ursache, das dynamische Magnetfeld der Sonne. In den tiefsten Schichten der Konvektionszone, etwa $200.000 \mathrm{~km}$ unter der Photosphäre, wird kinetische Energie aus der Konvektion und differentiellen Rotation der Sonne in magnetische Energie umgewandelt. Durch diesen als Dynamo bezeichneten Prozess werden Schläuche von Magnetfeld in der unteren Konvektionszone gebildet. Der Auftrieb in der Konvektionszone führt zu dem Aufsteigen von Flussröhren, die in ihren Durchstoßpunkten der Photosphäre als solare Aktivität beobachtet werden.

Sonnenflecken Zuerst bemerkt wurde das Magnetfeld der Sonne von Hale (1908), der die Polarisierung der aufgespaltenen Spektrallinien in Sonnenflecken untersuchte. Sonnenflecken besitzen mit bis zu 4000 Gauss (entsprechend 0,4 Tesla) die stärksten Magnetfelder auf der Sonne. Sie bestehen aus einem dunklen Kern, der wegen unterbundener Konvektion $2.000 \mathrm{~K}$ kälteren Umbra, umgeben von einem Ring aus radial verlaufenden magnetischen Strukturen, den penumbralen Filamenten. Der Durchmesser der Umbra kann bis zu $50.000 \mathrm{~km}$ betragen. Sonnenflecken treten zwischen $\pm 40^{\circ}$ heliographischer Breite auf, wobei sich innerhalb eines 11-jährigen Aktivitätszyklus der Sonne die Regionen der Entstehung neuer Sonnenflecken zu geringeren Breiten verschieben. Nach Ablauf eines solchen Zyklus vollzieht sich eine Umpolung aller solaren Magnetfelder, so dass der komplette, magnetische Aktivitätszyklus der Sonne 22 Jahre dauert. Die Lebensdauer eines einzelnen Sonnenfleckes liegt zwischen einigen Tagen und einem Monat, die Dauer des Zerfalls beträgt oft nur wenige Tage (Howard 1996).

Bipolare Gruppe Sonnenflecken treten häufig in Gruppen auf, die zwei Hauptflecken mit entgegengesetzter Polarität beinhalten, wobei in einer Hemisphäre fast immer die gleiche Polarität die anführende ist. Solche Gruppen werden bipolare Gruppen genannt. Die anführenden Polaritäten sind auf der Nord- und Südhälfte der Sonne entgegengesetzt. Mit der Umpolung der Magnetfelder nach Ablauf des 11-jährigen Zyklus werden die bipolaren Gruppen der Nordhälfte von der ehemalig führenden Polarität der Südhälfte angeführt und vice versa.

Poren Poren sind die kleinere Version der Sonnenflecken, sie unterscheiden sich jedoch dadurch, dass sie keine Penumbra besitzen. Die Magnetfelder der Poren sind immer noch stark genug, um die Konvektion zu unterbinden, so dass auch sie durch verstärkte radiative Abstrahlung kühler und dunkler als die Photosphäre erscheinen. Poren weisen typische Größen von 1000 
bis $4000 \mathrm{~km}$, gelegentlich auch $6000 \mathrm{~km}$ auf und beherbergen magnetische Feldstärken von etwa 2000 G (Sütterlin|1996).

Fackeln Fackeln sind Gebiete, die sich im Vergleich zu der ruhigen Sonne durch eine um etwa 30 \% erhöhte Intensität auszeichnen (Auffret \& Muller 1991) und hauptsächlich in der Nähe von Sonnenflecken, gelegentlich jedoch auch unabhängig von ihnen auftreten. Fackelgebiete werden hauptsächlich am Sonnenrand gefunden, da hier aufgrund des schrägen Blickwinkels zur Sonnenoberfläche in bis zu $200 \mathrm{~km}$ höheren Schichten beobachtet wird. Die Helligkeit der Fackeln entsteht dann durch eine im Gegensatz zu der umgebenden Granulation erhöhte Temperatur der einzelnen Fackelpunkte in diesen höheren Schichten der Sonne. Fackelpunkte sind der Sitz starker Magnetfelder im kG Bereich. Sie besitzen einen Durchmesser von etwa 100 bis $200 \mathrm{~km}$ und es wird vermutet, dass sie aus einzelnen, noch dünneren Flussröhren zusammengesetzt sind (de Boer \& Kneer 1992). Auch die Granulation eines Fackelgebietes unterscheidet sich von normaler Granulation. Die Granulen sind kleiner und zeigen eine längere Lebensdauer.

Plages Plages werden von Stellmacher \& Wiehr (2001) als die Gegenstücke der Fackeln in Scheibenmitte beschrieben. Sie treten ebenfalls als Anhäufungen einzelner, sehr kleiner magnetischer Elemente auf, von denen jedes eine mittlere Feldstärke von mehr als $1000 \mathrm{G}$ in der Photosphäre besitzt (Martínez Pillet et al. 1997). Diese kleinen Elemente, die sich in der Intensität im Gegensatz zu Fackelpunkten nicht gegen die Granulation absetzen, werden Filigree genannt. Der Übergang von Filigree in der Scheibenmitte zu Fackelpunkten am Sonnenrand ist noch ungeklärt. Plage-Gebiete treten genau wie Fackeln begleitend zu Sonnenflecken auf und enthalten anomale Granulation, die weniger Kontrast und weniger zeitliche Variation zeigt als normale. Der Zerfall von Plage-Regionen geschieht nach Howard (1996) langsam, er erstreckt sich über Wochen oder sogar Monate. Der ebenfalls 11-jährige Aktivitätszyklus von Plage-Regionen ist gegen den Zyklus der Sonnenflecken leicht phasenverschoben (Foukal 1993).

Azimutale Zentren Azimutale Zentren wurden zuerst von Martínez Pillet et al. (1997) entdeckt. Es handelt sich hierbei um Plage-Gebiete mit Strukturen, die genau wie Poren eine kontinuierliche Verteilung von Feldlinien aller Richtungen zeigen. Diese Strukturen verhalten sich ähnlich wie Poren oder Sonnenflecken, zeigen jedoch nicht die typische Verdunkelung im Kontinuum. Die azimutalen Zentren besitzen konzentriertere, größere Magnetfelder als normale PlageGebiete und weisen im allgemeinen auch höhere Füllfaktoren auf.

Flussröhren Flussröhren sind die kleinsten angenommenen magnetischen Elemente. Sie beschreiben einzelne, abgeschlossene Magnetfeldstränge. Ob Fackelpunkte, Filigree oder auch die penumbralen Filamente bereits diese gesuchten Flussröhren darstellen oder noch jeweils aus mehreren Flussröhren zusammengefasst bestehen, ist immer noch ungeklärt. 


\section{Einleitung}

Chromosphärisches Magnetfeld Die photosphärisch gemessenen Magnetfelder reichen weit in die Chromosphäre hinein und dehnen sich wegen des mit der Höhe abnehmenden Gasdruckes aus, so dass es zu der Ausbildung eines Baldachin (engl. Canopy) kommt, der sich auch mit über die ruhige Photosphäre ausbreitet Rüedi et al. (1995). Es wird vermutet, dass die gesamte Chromosphäre mit Magnetfeld ausgefüllt ist.

Koronales Magnetfeld In der noch höheren Schicht der Korona, die sich bis zu zehn Sonnenradien und mehr über die Photosphäre erstreckt, dominiert das Magnetfeld aufgrund des geringen Gasdruckes die Materie derart, dass diese sich ausschliesslich entlang der Feldlinien bewegt.

Protuberanzen Am Sonnenrand können koronale Magnetfelder, die bogenförmig über den aktiven Gebieten liegen, beobachtet werden. Diese starken Magnetfelder heben sich auf $200.000 \mathrm{~km}$ Länge bis zu $40.000 \mathrm{~km}$ hoch über die Photosphäre hinaus und beherbergen abströmende Materie, die von kondensierender Materie aus der Korona ersetzt wird. In Senken der Magnetfeldbögen sammelt sich Materie an, die im Licht der Ho-Linie in Emission beobachtet werden kann. Dieses Phänomen wird Protuberanz genannt. Die Lebensdauern von Protuberanzen reichen von einigen Monaten bis zu einem Jahr. Über der Sonnenscheibe sind Protuberanzen in Absorption zu sehen und werden Filamente genannt.

\subsection{Kleinskalige Magnetfelder}

Durch den Einsatz immer größerer Teleskope wurde es möglich, immer hochaufgelöstere Bilder der Sonne auszuwerten und immer kleinskaligere Strukturen zu untersuchen. Doch je besser die Auflösung, desto kleinere Strukturen wurden entdeckt, so dass auch heute noch die kleinsten Strukturen von größtem Interesse sind. Da das Ziel dieser Arbeit ein Beitrag zu der Erforschung der kleinskaligen Magnetfelder sein soll, werden diese nun etwas ausführlicher beschrieben.

\subsubsection{Entstehung und Lebensdauer}

Weder die Entstehung noch die Lebensdauer kleinskaliger magnetischer Strukturen sind derzeit mit Sicherheit bekannt. Fest steht, dass die Lebensdauer magnetischer Konstellationen mit dem magnetischen Fluss, den sie enthalten, abnimmt. Für eine einzelne Flussröhre konnte sie noch nicht bestimmt werden, wird jedoch von Solanki (1993) auf mindestens eine Stunde abgeschätzt.

Kleinskalige magnetische Strukturen finden sich vor allem in aktiven Gebieten, jedoch auch in der ruhigen Granulation in den Rändern und Ecken der Supergranulationszellen bzw. des chromosphärischen Netzwerkes. Die größeren aktiven Gebiete entstehen durch das Aufsteigen von Flussschläuchen aus der unteren Konvektionszone in die Photosphäre, wo sie sich zunächst als bipolare Gruppen manifestieren. Zu der Entstehung neuer Flussröhren gibt es verschiedene Theorien. 
Tarbell et al. (1990) beschreiben, dass hierbei zunächst kurzzeitige Aufströmungen von 0,6 km/s gemessen werden. Danach bilden sich helle Strukturen und eine starke Abströmung von $1 \mathrm{~km} / \mathrm{s}$ setzt ein, begleitet von der Entstehung der Flussröhre. Abströmung und auch Zuwachs an magnetischen Fluss halten für 30 bis 60 Minuten an. Durch die Konvektion werden die Flussröhren an den Kanten der Granulen und Supergranulen im Intergranulum zusammengeschoben.

Kleinskalige Magnetfelder werden aber nicht nur in neu entstehenden, aktiven Gebieten beobachtet. Auch alte, zerfallende Gebiete können einzelne, abgespaltene Flussröhren hinterlassen.

\subsubsection{Kontrast und Temperatur}

Am Sonnenrand zeigen Fackeln auch bei mittlerer Auflösung eine deutlich erhöhte Intensität, während die ihnen entsprechenden Filigree in der Scheibenmitte nur mit hochauflösenden Instrumenten zu erkennen sind. Da durch den schrägen Blickwinkel auf die Sonnenoberfläche am Sonnenrand die Sichtlinie eine längere Wegstrecke durch die Atmosphäre der Sonne zurücklegt als bei senkrechtem Blickwinkel, wird die optische Tiefe $\tau=1$ bereits in einer größeren Höhe der Sonnenatmosphäre erreicht.

Hochaufgelöste Beobachtungen zeigen nun einen Anstieg der relativen Fackelintensität von der Scheibenmitte zum Rand 1 , die bei etwa $\cos \theta=0,3$ ein Maximum mit Intensitäten von 1,2 bis 1,4 $I_{c}$ erreicht. ${ }^{2}$ Auffret \& Muller (1991) untersuchen kleinskalige magnetische Strukturen und finden für die hellsten, etwas größeren Strukturen von $0,4^{\prime \prime}$ bis $0,7^{\prime \prime}$, die auch am Sonnenrand noch detektiert werden können, einen Kontrastanstieg von $22 \%$ in Scheibenmitte zu $40 \%$ bei $\cos \theta=0,4$. Weiter zum Sonnenrand hin wurde bis $\cos \theta=0,15$ ein Abfall des Kontrastes festgestellt. Auch andere Autoren, z.B. Adjabshirizadeh \& Koutchmy (2002), erhalten ähnliche Ergebnisse, die Positionen des maximalen Kontrastes variieren hierbei zwischen 0,15 und 0,3. Sütterlin et al. (1999) stellen jedoch einen weiteren Kontrastanstieg bis zu $\cos \theta=0,1$ fest.

Warum die kleinsten magnetischen Strukturen im Kontrast zu der umgebenden Granulation am Sonnenrand sehr deutlich und in Scheibenmitte unauffällig sind, ist noch nicht eindeutig geklärt, und es ist noch kein Modell gefunden worden, das beide Verhaltensweisen gleichermaßen gut erklärt. Eine mögliche Erklärung wäre, dass die kleinen magnetischen Flusselemente bei der unzureichenden Auflösung heutiger Sonnenteleskope im Kontinuum der intergranularen Gebiete in Scheibenmitte unauffällig sind (Berger \& Title 1996), während man am Sonnenrand ausgedehntere, höher gelegene Strukturen sieht.

Einige Modelle, die versuchen den Übergang von Filigree zu Fackelpunkten zu beschreiben, sollen hier kurz genannt werden:

\footnotetext{
${ }^{1}$ Auch Poren zeigen eine Mitte-Rand-Variation ihres Kontrastes zu der umgebenden Granulation, der jedoch zum Sonnenrand hin abnimmt und sich möglicherweise sogar umkehrt (Sütterlin 1996). Poren sollten demnach in der Nähe des Sonnenrandes nur noch durch ihr Magnetfeld erkennbar sein, ähnlich den von Martínez Pillet et al. (1997) beschriebenen Azimuth Centers.

${ }^{2}$ Hier ist $\theta$ der Winkel zwischen der Normalen zur Sonnenoberfläche und der Strahlrichtung. Es ist also $\sin \theta=\frac{r}{R_{\odot}}$, wobei $R_{\odot}$ den Sonnenradius bezeichnet und $r$ den Abstand des Beobachtungsortes von der Scheibenmitte.
} 


\section{Einleitung}

Das Hot Wall Modell, das Spruit (1976) vorstellt, basiert auf stationären Flussröhren, die wegen eines geringeren internen Gasdruckes auch für Fackelpunkte eine Iso- $\tau$ Depression von etwa $100 \mathrm{~km}$ zeigen. Die Wände dieser Depression, durch die die Strahlung von der umgebenden Photosphäre nach innen dringt, um das Energiegleichgewicht zu erhalten, werden für den erhöhten Kontrast der Fackelpunkte am Sonnenrand verantwortlich gemacht. Das Hot Wall Modell sagt einen geringen Kontrast für die Scheibenmitte und einen leichten Kontrastanstieg zum Sonnenrand vorher, quantitativ kann es jedoch die starke Kontrastdifferenz zwischen Sonnenmitte und Sonnenrand für Fackeln nicht beschreiben.

Das Hot Cloud Modell von Deinzer et al.(1984) führt einen zusätzlichen Heizungsprozess in den höheren Schichten ein, der eine Wolke heisser Materie über der Flussröhre entstehen lässt. So ergeben sich signifikant höhere Kontraste bis zu 22\% am Sonnenrand, die allerdings einhergehen mit ebenfalls erhöhten Kontrasten von $10 \%$ in der Scheibenmitte.

Das Hillock Modell von Schatten et al. (1986) beschreibt das Aufsteigen von Materie und Energie (die als neutraler Wasserstoff in Sonnenflecken bis zur unteren Konvektionszone abgesunken sind) in Form von ionisiertem Wasserstoff in Fackeln, der durch Rekombination einen Temperaturanstieg direkt unter der Oberfläche der Sonne bewirkt. Durch den Temperaturanstieg expandiert das Gas und bildet durch Auftrieb kleine Hügelchen (engl. hillocks) in der Iso- $\tau$ Linie aus. Diese Vergrößerung der Sonnenoberfläche erlaubt so den Ausgleich des Strahlungsdefizites in Sonnenflecken. Der Temperaturanstieg unter der Oberfläche bewirkt eine Erhöhung des Kontrastes bis zum extremen Sonnenrand. In diesem Modell ist die Druckskalenhöhe in der Atmosphäre von Fackeln größer, was zu den Messungen von Stellmacher \& Wiehr (2001) passt. Der Kontrast der Fackelpunkte würde hier bis zum extremen Sonnenrand stetig zunehmen.

Eine Untersuchung des Hillock Modells von Sütterlin et al. (1999) ergab, dass der Kontrast der Fackelpunkte mit zunehmender Nähe des Sonnenrandes bis $\cos \theta=0,1$ ansteigt. Ob es jedoch auf dem extremen Rand mit $\cos \theta<0,1$ im Widerspruch zu dem Hillock Modell noch einmal zu einer Kontrastabnahme kommt, konnte nicht eindeutig geklärt werden.

Der hohe Kontrast, der bei Fackeln am Sonnenrand beobachtet wird, weist darauf hin, dass Fackeln in höheren Schichten heißer sind als ihre Umgebung. In einer Region bei $\cos \theta=0,55$ bestimmen Sütterlin et al. (1999) den Kontrast von 250 Fackelpunkten bei einer Wellenlänge von $550 \mathrm{~nm}$ zu 1.32 bis 1.43. Durch Messungen in drei Farbbändern, blau, rot und infrarot, werden höhenabhängige Intensitäten gewonnen, aus deren Differenzen Rückschlüsse auf die herrschenden Temperaturen möglich sind. So wurden die Temperaturen der Fackelpunkte zu 150 bis $450 \mathrm{~K}$ über dem Mittelwert der ungestörten Umgebung bestimmt. Für einen einzelnen Fackelpunkt wurde eine Extremtemperatur von $700 \mathrm{~K}$ über dem Mittelwert gefunden.

\subsubsection{Magnetfeld und Inklination}

Das Standardmodell kleinskaliger magnetischer Elemente, von denen jedes eine mittlere Feldstärke von mehr als 1000 Gauss in der Photosphäre besitzt, besagt, dass sich die Feldlinien schnell mit der Höhe auffächern und ein Canopy von deutlich geringerer Feldstärke über der 
Photosphäre ausbilden (Martínez Pillet et al. 1997). Obwohl dieses Modell in vielen Bereichen korrekt zu sein scheint, enthält es doch noch Unklarheiten. Flussröhren können selbst mit den besten Beobachtungen noch nicht räumlich aufgelöst werden. Es ist sehr wenig über die VektorEigenschaften des Magnetfeldes und seine Dynamik bekannt, und Plages, die nicht in Sonnenmitte liegen, sind bisher wenig erforscht.

Der Kontrast und die Intensität einer Struktur können nicht direkt als Indikatoren für das Magnetfeld angesehen werden (Berger \& Title 1996). Doch nicht nur der Kontrast, auch das Magnetfeld zeigt eine Mitte-Rand-Variation über die Sonnenscheibe. Es existiert eine kleine Komponente schwächerer Magnetfelder mit Stärken von weniger als $1000 \mathrm{G}$ in Scheibenmitte, die häufig bogenartig die Fusspunkte verschiedener Polaritäten verbinden. Die am häufigsten vertretene Magnetfeldstärke in kleinskaligen magnetischen Elementen der Scheibenmitte beträgt jedoch 1400 G mit einem mittleren Füllfaktor von $15 \%$ (Martínez Pillet et al. 1997). Von der Scheibenmitte zum Sonnenrand nimmt diese Magnetfeldstärke von $1400 \mathrm{G}$ auf $800 \mathrm{G}$ ab. Da das Licht des Sonnenrandes aus einer etwa 200 km höher gelegenen Schicht der Atmosphäre stammt, leiten Martínez Pillet et al. (1997) hieraus einen mittleren Gradient von minus $3 \mathrm{G} / \mathrm{km} \mathrm{ab}^{3}$

Sie zeigen, dass die magnetischen Feldlinien über die gesamte Höhendifferenz von $200 \mathrm{~km}$ größtenteils vertikal verlaufen. Es werden Inklinationen von weniger als $10^{\circ}$ und keine bevorzugte azimutale Orientierung festgestellt. Stellmacher \& Wiehr (2001) untersuchen die Inklination der Feldlinien von Fackelpunkten in Spektrallinien unterschiedlicher Entstehungshöhe und erhalten eine örtliche Verschiebung der erhöhten Intensität in Linien, die höher entstehen, nicht jedoch die Beteiligung einer größeren Anzahl von Ortspunkten als im Kontinuum. Hieraus und aus dem Fehlen von Stokes- $V$-Signalen (vgl. Kap. 2.3), die nur die Sichtlinienkomponente des Magnetfeldes zeigen, die bei vertikalen Magnetfeldern am Sonnenrand nicht vorhanden ist, schließen sie, dass Fackeln sich bis zu einer Höhe von mehr als $300 \mathrm{~km}$ über der Photosphäre nicht auffächern. In größeren Höhen, über dem Temperaturminimum, werden Stokes- $V$-Signale gemessen, folglich existiert hier eine Aufweitung der Flussröhren.

\subsubsection{Geschwindigkeiten}

Horizontale Geschwindigkeiten auf großen Skalen sind leicht zu bestimmen. So bewegen sich Magnetfelder unterschiedlicher Polarität mit einer Geschwindigkeit von 0,5 bis $1 \mathrm{~km} / \mathrm{s}$ aufeinander zu (Solanki 1993), kleinskalige magnetische Strukturen in der Nähe eines gleich gepolten Sonnenfleckes bewegen sich mit bis zu $1,5 \mathrm{~km} / \mathrm{s}$ von ihm weg. Selbst die kleinsten, hochaufgelösten magnetischen Strukturen befinden sich ständig in horizontaler Bewegung (de Boer \& Kneer 1992).

Betrachtet man die zeitliche Entwicklung eines aktiven Gebietes, so beobachtet man bei der Entstehung kleinskaliger, magnetischer Strukturen eine Aufströmung, gefolgt von einer Abströmung mit bis zu $2 \mathrm{~km} / \mathrm{s}$. Eine mögliche Erklärung dieser Abströmung wäre der Abfluss der Materie

\footnotetext{
${ }^{3}$ Der Feldstärkegradient über Poren beträgt laut Sütterlin (1996) etwa 4 G/km. In der Chromosphäre nimmt der Gradient ab, über Sonnenfleckenumbren messen Rüedi et al. (1995) noch ca. 0,5 G/km.
} 


\section{Einleitung}

durch die gerade entstandene Flussröhre. Beobachtungen zeigen jedoch, dass Abströmungen nicht im Stokes- $V$-Signal, sondern ausschliesslich in Stokes- $I$ zu sehen sind. Daraus läßt sich folgern, dass die Materie-Abströmungen vermutlich außerhalb der magnetischen Strukturen stattfinden.

In frühen Beobachtungen (1971 bis 1985) wurden häufig abwärtsgerichtete Geschwindigkeiten in magnetischen Elementen detektiert, neuere Messungen zeigen im Mittel jedoch gar keine Geschwindigkeiten im Magnetfeld. Solanki (1993) erklärt dieses dadurch, dass in den früheren Messungen das zeitliche Mittel oder die Stichprobe der Strukturen zu gering gewählt wurde, oder die spektrale Auflösung der Daten nicht gut genug war. Es wird gefolgert, dass es keine Anzeichen für stationäre Strömungen in der Mehrheit der kleinskaligen magnetischen Strukturen gibt. Trotzdem wird das Vorhandensein stationärer Strömungen von Solanki (1993) erwartet. Die Breite der Stokes- $V$-Profile zeigt, dass in den erzeugenden magnetischen Elementen Geschwindigkeiten existieren. Diese Geschwindigkeiten bestehen somit entweder in Form von Schwingungen oder Wellen, die im zeitlichen oder räumlichen Mittel nicht sichtbar wären, oder in der Form stationärer Strömungen in kleineren Elementen, die bisher nicht aufgelöst werden.

\subsubsection{Heizung höherer Schichten}

Kleine magnetische Strukturen erhöhen den Energieausfluss aus der Sonnenatmosphäre der unteren Photosphäre, weil sie Energie von der umgebenden Granulation in Form von Strahlung absaugen. Dieses geschieht folgendermaßen (Solanki 1993): Die geringere Dichte des Gases in der Flussröhre führt zu einer Wilson Depression, d.h. die $\tau=1$ Linie liegt in der Flussröhre tiefer als in der umgebenden Granulation. Photonen, die über der $\tau=1$ Linie der Flussröhre, aber unterhalb der $\tau=1$ Linie der Granulation durch eine Seitenwand in die Flussröhre eindringen, entkommen mit einer größeren Wahrscheinlichkeit als Photonen eines vergleichbaren feldfreien Mediums. Dies bedeutet, dass Flussröhren die Oberfläche vergrößern, durch die die Photonen der Photosphäre entkommen können. Flussröhren verändern so die Richtung der Strahlung und kühlen die sie direkt umgebenden Granulen ab.

Der horizontale Energiefluss findet in der unteren Photosphäre vor allem von der Granulation durch die Flussröhrenwand statt. Innerhalb der Flussröhre ist die Konvektion zum größten Teil unterdrückt, so dass der vertikale Energietransport hauptsächlich durch Strahlung geschieht. In höheren Schichten, in denen die Flussröhre im Vergleich zur Umgebung heißer ist, dreht sich dieser Fluss um. Die Strahlung erfolgt hier von der Flussröhre in die Umgebung.

Auf diese Weise heizen kleinskalige magnetische Strukturen die höheren Schichten und erhöhen so auch die Strahlung aus diesen Schichten. Diese Heizung wird durch die Absorption des erhöhten Strahlungsflusses, aber auch durch die Fortpflanzung von Wellen oder die Wiedervereinigung (engl. reconnection) von Feldlinien angetrieben. 


\subsubsection{Schwingungen und Wellen}

Ein kleiner Teil der von der Granulation an die Flussröhre abgegebenen Energie ist auch kinetische Energie, denn die Flussröhren werden durch die sich ändernden Strömungsrichtungen in der Granulation, eventuell auch durch Schockwellen angestoßen. Diese Stöße können longitudinale, akustische Wellen oder transversale Kink-Wellen anregen, während Wirbelströmungen um einzelne Flussröhren herum rotierende Alfvén Wellen bewirken. Hierbei geht die kinetische Energie von der Granulation auf die Flussröhre über, die weiter geleitet wird in die höheren Schichten der Atmosphäre und hier eine Heizung verursacht.

Die Mode der Kink-Wellen ist für den Energietransport in die äußere Atmosphäre vermutlich wichtiger als Alfvén oder longitudinale Wellen, da Kink Wellen durch die Bewegungen der Granulation leichter angeregt werden können (Solanki 1993).

Solare Schwingungen werden nicht in allen Frequenzen und allen Strukturen gleich stark beobachtet. So treten nach Solanki (1993) Schwingungen mit kleinen Frequenzen in Plage-Gebieten und dem Netzwerk schwächer auf als Schwingungen großer Frequenzen. Dieses ist für die Untersuchung kleinskaliger, magnetischer Strukturen, wie sie vor allem in Plage-Gebieten vorkommen, von besonderem Interesse, denn es bedeutet, dass die globale 5-Minuten-Oszillation der Sonne in Plages deutlich schwächer zu sehen ist als in der ruhigen Sonne. 
1 Einleitung 


\section{Theoretische Grundlagen}

Für Messung und Verständnis der Auswirkungen eines Magnetfeldes auf die Lichtausbreitung ist eine theoretische Beschreibung derselben unerlässlich. Die Grundlagen der Entstehung, Ausbreitung und Messung polarisierten Lichtes sollen daher Inhalt des folgenden Kapitels sein.

Zuvor soll noch eine kurze, aber wichtige Anmerkung eingeschoben werden: In der Astrophysik hat es sich eingebürgert, die magnetische Flussdichte $\vec{B}$ fälschlich mit magnetischer Feldstärke zu benennen. Die tatsächliche magnetische Feldstärke $\vec{H}$, die mit der Flussdichte $\vec{B}$ über

$$
\vec{B}=\mu \mu_{0} \vec{H}
$$

zusammenhängt, wird nicht direkt gemessen und häufig, wie auch hier, wird bei der Behandlung solarer Magnetfelder das Gauss'sche Maßsystem verwendet. In den Einheiten des Gauss'schen Systems besitzen die Induktionskonstante $\mu_{0}$ und auch die Permeabilität $\mu$ in sehr guter Näherung Werte von eins, so dass sich Feldstärke und Flussdichte hier nur durch die Einheit unterscheiden. Man möge daher die laxe Benennung der magnetischen Flussdichte als Feldstärke nachsehen. Die Einheiten aller angegebenen Flussdichten $\vec{B}_{\|}$stehen korrekt in Tesla oder Gauss (mit $10^{4} \mathrm{G}$ $=1 \mathrm{~T}$ ).

\subsection{Zeeman-Effekt}

Liegt an dem Entstehungsort einer Spektrallinie ein Magnetfeld vor, so wirkt sich dieses, sofern es sich um eine magnetisch sensitive Linie handelt, in einer Aufspaltung der zu Grunde liegenden Energieniveaus aus. Aus der Zeeman-Aufspaltung der Spektrallinie lassen sich daher Informationen über das Magnetfeld am Entstehungsort zurückgewinnen. Stärkere Magnetfelder von mehr als 0,15 T können direkt anhand der Aufspaltung durch den Zeeman-Effekt bestimmt werden, bei schwächeren Feldern muss auf die mit der Aufspaltung einhergehende Polarisation der einzelnen Komponenten der Linie zurückgegriffen werden. Der Zeeman-Effekt wird beschrieben in Haken \& Wolf (1993), Solanki (1993) und Stix (1989).

\subsubsection{Aufspaltung der Zeeman-Komponenten}

Jedes zu einem Atom gehörende Elektron besitzt einen Spin $s$ und einen Bahndrehimpuls $l$. In Abwesenheit eines Magnetfeldes besitzt das Atom Energieniveaus, die sich in Abhängigkeit von dem Gesamtdrehimpuls $J$, der sich aus allen $s$ und $l$ zusammensetzt, bilden. Wenn das betrachtete 


\section{Theoretische Grundlagen}

Atom jedoch einem Magnetfeld ausgesetzt wird, spalten die Energieniveaus auf. Diese Aufspaltung wird nach ihrem Entdecker Zeeman-Effekt genannt.

In einem schwachen Magnetfeld koppeln die Bahndrehimpulse $l$ aller Elektronen zum Gesamtbahndrehimpuls $L$ und die Spins $s$ zum Gesamtspin $S, L S$-Kopplung genannt. Alternativ zu der $L S$-Kopplung existiert auch eine $j j-$ Kopplung, bei der die Spins und Bahndrehimpulse je eines Elektrons $j=s+l$ koppeln. Reine $j j-$ Kopplung tritt jedoch nur bei schwereren Atomen und starken Magnetfeldern auf. Selbst die stärkeren auf der Sonne vorkommenden Magnetfelder von etwa 0,3 Tesla sind für die meisten Linien noch schwach im Sinne der Kopplung, so dass im Folgenden nur die hierfür geltende $L S$-Kopplung beschrieben werden soll.

Die Energie $E_{J, M}$ der Unterniveaus läßt sich aus der Energie des unaufgespaltenen Niveaus $E_{J}$ bei einem Magnetfeld der Stärke $B$ berechnen durch

$$
E_{J, M}=E_{J}+\mu_{0} g M_{J} B
$$

wobei die magnetische Quantenzahl $M_{J}$ die zum Magnetfeld $B$ parallele Komponente des Gesamtdrehimpulses $J$ darstellt $\left(-J \leq M_{J} \leq J\right)$ und $\mu_{0}=\frac{e h}{4 \pi m_{e} c}$ das Bohr'sche Magneton mit der Elementarladung $e=1,6021 \cdot 10^{-19} \mathrm{C}$, der Elektronruhemasse $m_{e}=9,1091 \cdot 10^{-31} \mathrm{~kg}$, der Lichtgeschwindigkeit $c=2,99792 \cdot 10^{8} \mathrm{~m} / \mathrm{s}$ und der Planck-Konstanten $h=6,626 \cdot 10^{-34} \mathrm{~J} / \mathrm{s} . g$ bezeichnet den Landé-Faktor, ein Maß für die Weite der Aufspaltung, der sich aus

$$
g=1+\frac{J(J+1)+S(S+1)-L(L+1)}{2 J(J+1)}
$$

ergibt.

Durch die Aufspaltung der atomaren Energieniveaus werden neue Übergänge möglich, so dass auch die Spektrallinie in Teilkomponenten aufgespalten wird. Da diese Aufspaltung direkt proportional zu der magnetischen Feldstärke $B$ ist, kann sie zur Messung des am Entstehungsort der Linie anliegenden Feldes benutzt werden. Der Zusammenhang zwischen der Wellenlängenaufspaltung $\Delta \lambda_{H}$ und der magnetischen Feldstärke $B$ ist gegeben durch

$$
\Delta \lambda_{H}=\frac{e}{4 \pi m_{e} c} \lambda^{2} g^{*} B
$$

Der Faktor $g^{*}=g M_{J}-g^{\prime} M_{J}^{\prime}$ berechnet sich aus den Landé-Faktoren $g$ und $g^{\prime}$, die die Aufspaltung der beteiligten Energieniveaus beschreiben, und den zugehörigen magnetischen Quantenzahlen $M_{J}$, wobei $\Delta M=M_{J}-M_{J}^{\prime}=0$ oder \pm 1 gelten muss.

\section{Normaler und anomaler Zeeman-Effekt}

Die einfachste Möglichkeit, die Aufspaltung einer Spektrallinie in genau drei Teillinien, wird normaler Zeeman-Effekt genannt. Der normale Zeeman-Effekt tritt jedoch nur unter bestimmten Umständen auf: Wenn es sich um Übergänge zwischen $J=0$ und $J=1$ handelt, werden genau 
drei Teillinien, Zeeman-Triplett genannt, gebildet, da das $J=0$-Energieniveau nicht aufspalten kann. Eine zweite Möglichkeit ist die Beteiligung zweier Energieniveaus mit gleichem LandéFaktor, denn dann gilt $g^{*}=g$ und es existiert nur ein Landé-Faktor für die gesamte Linie.

Im allgemeineren Fall des anomalen Zeeman-Effektes spaltet die Spektrallinie in viele Teilkomponenten auf, in ein Zeeman-Multiplett. Man definiert in diesem Fall für den Schwerpunkt der verschobenen Komponenten einen effektiven Landé-Faktor $g_{\text {eff }}$, der dem Landé-Faktor eines Zeeman-Tripletts entspricht, dessen Komponenten die gleiche Aufspaltung zeigen wie die Schwerpunkte des Zeeman-Multipletts. Für die Berechnung der Magnetfeldstärke kann in diesem Fall $g^{*}=g_{\text {eff }}$ benutzt werden.

Eine umfassende Tabelle wird von Charlotte Moore (1966) gestellt, die jeder solaren Spektrallinie eine Multiplettnummer zuteilt. Für die hier benutzte neutrale Eisenlinie bei 6302,5 die Multiplettnummer 816 angegeben, die Fe II Linie bei 6149,2 $\AA$ besitzt die Nummer 74. In einer zweiten Tabelle von Moore (1945) finden sich die atomaren Übergänge zu den Multiplettnummern, aus denen sich nach Formel 2.2 die Landé-Faktoren berechnen lassen.

Bei der Fe I Linie bei $6302,5 \AA$ handelt es sich um einen ${ }^{5} P_{1}-{ }^{5} D_{0}$ Übergang, und da das energetisch höhere Niveau mit $J=0$ nicht aufspaltet, um ein reines Zeeman-Triplett mit dem Landé-Faktor $g=2,5$. Die 6149, $2 \AA$ Linie besitzt einen ${ }^{4} P_{\frac{1}{2}}-{ }^{4} D_{\frac{1}{2}}$ Übergang mit $g_{\text {eff }}=\frac{4}{3}$. Eine umfangreiche Tabelle effektiver Landé-Faktoren findet sich bei Beckers (1969).

\subsubsection{Polarisation der Zeeman-Komponenten}

Jedes Zeeman-Multiplett besteht aus mehreren verschobenen und unverschobenen Komponenten. Die positiv und negativ verschobenen Komponenten mit $\Delta M= \pm 1$ werden $\sigma^{ \pm}$-Komponenten, die Komponenten mit $\Delta M=0$ werden $\pi$-Komponenten genannt.

Die $\sigma$-Komponenten zeichnen sich jedoch nicht nur durch ihre in der Regel größere Abspaltung von den $\pi$-Komponenten aus, abhängig von der Orientierung des Magnetfeldes werden diese Komponenten auch unterschiedlich polarisiert. Je nach Blickwinkel zum Magnetfeld unterscheidet man hier den longitudinalen und den transversalen Fall des Zeeman-Effektes.

Von longitudinalem Fall spricht man, wenn das Magnetfeld genau parallel zur Sichtlinie liegt. In diesem Fall ist das Licht der $\sigma^{ \pm}$-Komponenten entgegengesetzt zueinander zirkular polarisiert. Die $\pi$-Komponenten sind parallel zur Ausbreitungsrichtung linear polarisiert, so dass in der beobachteten Richtung keine Strahlung detektiert wird.

Der transversale Fall beschreibt das andere Extrem, ein Magnetfeld senkrecht zur Sichtlinie. Hier werden sowohl $\sigma$ - als auch $\pi$-Komponenten linear polarisiert empfangen, wobei in Absorptionslinien die $\pi$-Komponenten senkrecht zum Magnetfeld und die $\sigma^{ \pm}$-Komponenten parallel zum Magnetfeld polarisiert sind. Für Emissionslinien sind diese Polarisationsrichtungen um $90^{\circ}$ gedreht.

In dem allgemeineren Fall eines schrägen Blickwickels ergeben sich elliptisch polarisierte $\sigma^{ \pm}$ Komponenten und linear polarisierte $\pi$-Komponenten. 


\section{Theoretische Grundlagen}

Die Bedeutung der Polarisation der Zeeman-Komponenten liegt darin, dass sie sich auch dann noch messen lässt, wenn die Aufspaltung der Komponenten zu gering ist, als dass sie detektiert werden könnte. So können schwache Magnetfelder, die keine sichtbare Linienaufspaltung bewirken, durch die Beobachtung z.B. der zirkular polarisierten Komponenten des Lichtes untersucht werden, vgl. Kap. 2.3 .

\subsection{Dopplerbreite von Spektrallinien}

Um ein Magnetfeld mit Hilfe der Zeeman-Aufspaltung messen zu können, ist es notwendig, auch die Dopplerbreite der untersuchten Spektrallinien zu kennen. Denn auch eine starke ZeemanAufspaltung kann nicht für die Bestimmung des Magnetfeldes herangezogen werden, wenn die natürliche Breite der Linie in der gleichen Größenordnung liegt.

Die Dopplerverbreiterung von Absorptionslinien wird beschrieben nach Voigt (1991) und Stix (1989). Die Verminderung oder Vergrößerung der emittierten Wellenlänge eines sich bewegenden Atomes wird durch den Doppler-Effekt beschrieben. So emittiert ein sich auf den Beobachter zubewegendes Atom eine kürzere, blauverschobene Wellenlänge, ein sich wegbewegendes Atom eine rotverschobene. In einem heißen Gas, in dem sich alle Atome mit unterschiedlichen Geschwindigkeiten bewegen, entsteht daher abhängig von der Temperatur $T$ eine Verbreiterung der Spektrallinie. Diese Breite der Spektrallinie ergibt sich aus der mittleren thermischen Geschwindigkeit $v_{t}$

$$
v_{t}=\sqrt{\frac{2 R T}{\mu}}
$$

der Gasatome mit Atom- oder Molekülmasse $\mu . R=8,31 \mathrm{~J} / \mathrm{Kmol}$ bezeichnet die Gaskonstante. Somit erklärt sich z.B. die enorme Breite der Balmerlinien aus der geringen Atommasse des Wasserstoffs.

Die Dopplerverbreiterung $\Delta \lambda_{D}$ der Spektrallinie ist nun mit der Lichtgeschwindigkeit $c$ in Abhängigkeit von der thermischen Geschwindigkeit $v_{t}$ gegeben durch

$$
\Delta \lambda_{D}=\frac{\lambda}{c} \sqrt{\frac{2 R T}{\mu}} .
$$

Neben der Geschwindigkeitsabhängigkeit zeigt die Dopplerverbreiterung noch eine Abhängigkeit von der Wellenlänge $\lambda$ der Spektrallinie.

\section{Weak Field}

Ein Vergleich der Zeeman-Aufspaltung (Formel 2.3) mit der Dopplerverbreiterung (Formel 2.5) einer Absorptionslinie zeigt, dass die Dopplerverbreiterung $\Delta \lambda_{D}$ proportional zu $\lambda$ ist, während 
die Zeeman-Aufspaltung $\Delta \lambda_{H}$ quadratisch von der Wellenlänge abhängt. Die Zeeman-Aufspaltung ist daher bei schwachen Magnetfeldern im Infraroten deutlicher zu sehen als im sichtbaren Licht.

Das Verhältnis der Zeeman-Aufspaltung zur Dopplerbreite einer Linie bei einem gegebenen Magnetfeld wird benutzt, um Magnetfelder zu klassifizieren. So wird der Fall des schwachen Feldes (Weak Field) benannt, wenn $\Delta \lambda_{H} \ll \Delta \lambda_{D}$. Stark heisst das Magnetfeld, sofern $\Delta \lambda_{H} \gg$ $\Delta \lambda_{D}$ zutrifft. Diese Einteilung ist für einige Magnetfeldmessungen von größter Bedeutung, vgl. Kap. 4.4.3.

\subsection{Der Stokes-Vektor}

Für die Beschreibung polarisierten Lichtes wird in der Astrophysik allgemein der 1852 von G.G. Stokes eingeführte Formalismus benutzt, hier nach Solanki (1993) und Stix (1989) beschrieben. Die Vorteile des von Stokes eingeführten Vektors liegen zum einen in der Trennung der linear und zirkular polarisierten Anteile der Strahlung, zum anderen in seiner direkten Messbarkeit. Der sogenannte Stokes-Vektor besteht aus den vier Komponenten $I, Q, U$ und $V$, die definiert sind durch

$$
\vec{I}=\left[\begin{array}{c}
I \\
Q \\
U \\
V
\end{array}\right]:=\left[\begin{array}{c}
I_{\mathrm{u}}+I_{\mathrm{p}} \\
I_{\operatorname{lin}}\left(\chi=0^{\circ}\right)-I_{\operatorname{lin}}\left(\chi=90^{\circ}\right) \\
I_{\operatorname{lin}}\left(\chi=45^{\circ}\right)-I_{\text {lin }}\left(\chi=135^{\circ}\right) \\
I_{\text {zirk }}(\text { rechts })-I_{\text {zirk }}(\text { links })
\end{array}\right],
$$

wobei $I$ die Gesamtintensität sowohl der polarisierten Strahlung $I_{\mathrm{p}}$ als auch der unpolarisierten Strahlung $I_{\mathrm{u}}$ beschreibt. $Q$ und $U$ stellen die Anteile der linear polarisierten Strahlung dar, wobei die Winkel $\chi$ relativ zu einer von dem Beobachter definierten Referenzrichtung gemessen werden, und $V$ beschreibt den zirkular polarisierten Anteil des Lichtes. Diese anschauliche Definition ist gut für die Beobachtung geeignet, da sie einer praktischen Messanleitung für $I, Q, U$ und $V$ gleicht. Für die mathematische Beschreibung jedoch existiert eine alternative Definition, die dem Wellencharakter des elektromagnetischen Feldes Rechnung trägt.

\section{Mathematische Beschreibung}

Für einen einzelnen, monochromatischen Wellenzug elektromagnetischer Strahlung mit Ausbreitungsrichtung $z$ liegt der elektrische Feldvektor $\vec{E}$ in der $x, y$-Fläche und lässt sich schreiben als

$$
\vec{E}=\left[\begin{array}{c}
\xi_{x} \cos \phi \\
\xi_{y} \cos (\phi+\varepsilon)
\end{array}\right] \text {. }
$$

Hierbei ist die $x$-Komponente des Feldvektors durch ihre Amplitude $\xi_{x}$ in $x$-Richtung und eine Schwingung mit $\phi=\omega t-k z$ beschrieben, die $y$-Komponente analog durch ihre Amplitude $\xi_{y}$ und eine Schwingung gleicher Frequenz mit Phasenverschiebung $\varepsilon$. 


\section{Theoretische Grundlagen}

Da natürliches Licht nie perfekt monochromatisch ist, werden in der Definition von $I, Q, U$ und $V$ die zeitlichen Mittel der Größen verwendet. Die Stokes-Parameter lassen sich so beschreiben durch

$$
\begin{aligned}
I & =\left\langle\xi_{x}^{2}+\xi_{y}^{2}\right\rangle \\
Q & =\left\langle\xi_{x}^{2}-\xi_{y}^{2}\right\rangle \\
U & =\left\langle 2 \xi_{x} \xi_{y} \cos \varepsilon\right\rangle \\
V & =\left\langle 2 \xi_{x} \xi_{y} \sin \varepsilon\right\rangle
\end{aligned}
$$

In natürlichem, unpolarisiertem Licht besitzt der elektromagnetische Feldvektor $\vec{E}$ keine Vorzugsrichtung, so dass die Amplituden $\xi_{x}^{2}$ und $\xi_{y}^{2}$ im Mittel gleich groß sind. Auch sind alle Phasenverschiebungen $\varepsilon$ gleich wahrscheinlich, so dass $Q=U=V=0$ folgt. Das Licht wird in diesem Fall allein durch die Intensität $I$ charakterisiert.

Ein vollständig polarisiertes Wellenpaket dagegen würde sich durch eine feste Phasenverschiebung $\varepsilon$ und ein festes Verhältnis von $\xi_{x}$ zu $\xi_{y}$ auszeichnen. Für vollständig polarisiertes Licht gilt daher

$$
I^{2}=Q^{2}+U^{2}+V^{2}
$$

Im allgemeinen Fall ist das beobachtete Licht jedoch teilweise polarisiert. Es gilt

$$
I^{2} \geq Q^{2}+U^{2}+V^{2}
$$

und durch

$$
P=\sqrt{\frac{Q^{2}+U^{2}+V^{2}}{I^{2}}}
$$

lässt sich der Polarisationsgrad $P$ des Lichtes angeben.

Da die Stokes-Parameter wellenlängenabhängig sind, müssen für ihre Analyse die kompletten Wellenlängen-Profile $I(\lambda), Q(\lambda), U(\lambda), V(\lambda)$ verwendet werden.

Für eine vollständige Beschreibung des Magnetfeldes in Betrag und Richtung müssen alle vier Stokes-Parameter ausgewertet werden. Die Auswertung von Stokes- $V$-Signalen ausschließlich, wie sie in dieser Arbeit benutzt wird, liefert anstelle des vollen Magnetfeldvektors seine Sichtlinienkomponente. Alle Magnetfeldstärken, die im Folgenden angegeben sind, beziehen sich daher auch ohne den ausdrücklichen Verweis auf die Sichtlinienkomponente des Magnetfeldes.

\subsection{Strahlungstransport im Magnetfeld}

Um die Ausbreitung elektromagnetischer Strahlung in einem Medium zu beschreiben, das ein Magnetfeld enthält, reicht es nicht, die Absorptions- bzw. Emissionsprozesse der Gesamtintensität der Strahlung zu untersuchen. Die verschiedenen Polarisationszustände des Lichtes und 
damit die Parameter des Stokes-Vektors erfahren unterschiedliche Emissions- und Absorptionsprozesse während ihrer Ausbreitung entlang der Sichtlinie. Diese werden zusammengestellt nach Solanki (1993), Sütterlin (1996) und Landi Degl'Innocenti (1992).

Die Strahlungstransportgleichung für den Stokes-Vektor $\vec{I}_{v}$ des polarisierten Lichtes der Frequenz $v$ im lokalen thermodynamischen Gleichgewicht (LTE) unter Anwesenheit eines Magnetfeldes schreibt sich

$$
\frac{d \vec{I}_{v}}{d \tau}=\left(\mathbf{1}+\Omega_{v}\right)\left(\vec{I}_{v}-\vec{B}_{v}\right)
$$

Hierbei beschreibt $\frac{d \vec{I}_{V}}{d \tau}$ die Änderung der Stokes-Parameter mit der optischen Tiefe $\tau$. Die optische Tiefe ist definiert als

$$
\tau(z)=\int_{z}^{\infty} \kappa_{v}^{c}\left(z^{\prime}\right) d z^{\prime} \Leftrightarrow d \tau=-\kappa_{v}^{c} d z
$$

mit dem Kontinuums-Absorptionskoeffizienten $\kappa_{v}^{c}$ und der geometrischen Höhe $z$.

$\vec{I}_{v}$ beschreibt den Stokes-Vektor und $\vec{B}_{v}=\left(B_{v}, 0,0,0\right)^{\mathrm{T}}$ mit der Planckfunktion $B_{v}(T)$ die Quellfunktion. ()$^{\mathrm{T}}$ bezeichnet die Transponierte des Vektors. 1 beschreibt die Einheitsmatrix und $\Omega_{v}$ die Absorptionsmatrix mit

$$
\Omega_{v}=\left(\begin{array}{cccc}
\eta_{I} & \eta_{Q} & \eta_{U} & \eta_{V} \\
\eta_{Q} & \eta_{I} & \rho_{V} & -\rho_{U} \\
\eta_{U} & -\rho_{V} & \eta_{I} & \rho_{Q} \\
\eta_{V} & \rho_{U} & -\rho_{Q} & \eta_{I}
\end{array}\right)
$$

Hierbei bezeichnen $\eta_{i}, i \in[I, Q, U, V]$, die Absorptionskoeffizienten $\eta_{i}=\frac{\kappa_{i}^{l}}{\kappa^{c}}$ des Kontinuums $c$ und der Linie $l$ abhängig von dem Winkel $\gamma$ des Magnetfeldes zur Sichtlinie und dem azimutalen Winkel $\phi$.

$$
\begin{aligned}
& \eta_{I}=\frac{\eta_{o}}{2} \cdot \sin ^{2} \gamma+\frac{\eta_{+}+\eta_{-}}{4} \cdot\left(1+\cos ^{2} \gamma\right) \\
& \eta_{Q}=\left(\frac{\eta_{o}}{2}-\frac{\eta_{+}+\eta_{-}}{4}\right) \cdot \sin ^{2} \gamma \cdot \cos 2 \phi \\
& \eta_{U}=\left(\frac{\eta_{o}}{2}-\frac{\eta_{+}+\eta_{-}}{4}\right) \cdot \sin ^{2} \gamma \cdot \sin 2 \phi \\
& \eta_{V}=\frac{\eta-+\eta_{+}}{2} \cdot \cos \gamma
\end{aligned}
$$

Die Indizes $o, \pm$ beschreiben die Koeffizienten der $\pi$ und $\sigma^{ \pm}$Komponenten des Zeeman-Effektes, respektive $\eta_{ \pm}=\eta_{o}\left(\lambda \pm \Delta \lambda_{H}\right)$.

Die $\rho_{i}, i \in[Q, U, V]$ in der Absorptionsmatrix 2.14 beschreiben magnetooptische Effekte, die eine Rotation der Polarisationsebene des linear polarisierten Lichtes (Faraday-Rotation) und eine Phasenverzögerung (Voigt-Effekt) bewirken. Elektrodynamische Berechnung liefert 


\section{Theoretische Grundlagen}

$$
\begin{aligned}
& \rho_{Q}=\left(\frac{\rho_{o}}{2}-\frac{\rho_{+}+\rho_{-}}{4}\right) \cdot \sin ^{2} \gamma \cdot \cos 2 \phi \\
& \rho_{U}=\left(\frac{\rho_{o}}{2}-\frac{\rho_{+}+\rho_{-}}{4}\right) \cdot \sin ^{2} \gamma \cdot \sin 2 \phi \\
& \rho_{V}=c \frac{\rho_{-}-\rho_{+}}{2} \cdot \cos \gamma
\end{aligned}
$$

wobei analog zu $\eta$ gilt $\rho_{ \pm}=\rho_{o}\left(\lambda \pm \Delta \lambda_{H}\right)$.

Für den einfachsten Fall, in dem die Absorptionsmatrix $\Omega_{v}$ als unabhängig von der Höhe angenommen wird, und die Planckfunktion die Form $B_{v}=B_{v_{o}}\left(1+\beta_{o} \tau\right)$ besitzt, gibt es eine analytische Lösung der Strahlungstransportgleichung 2.12

$$
\begin{array}{ll}
I= & B_{v_{o}}\left(1+\beta_{o} \mu \frac{1+\eta_{I}}{\left(1+\eta_{I}\right)^{2}-\eta_{Q}^{2}-\eta_{U}^{2}-\eta_{V}^{2}}\right) \\
Q= & -B_{v_{o}} \beta_{o} \mu \frac{\eta_{Q}}{\left(1+\eta_{I}\right)^{2}-\eta_{Q}^{2}-\eta_{U}^{2}-\eta_{V}^{2}} \\
U= & -B_{v_{o}} \beta_{o} \mu \frac{\eta_{U}}{\left(1+\eta_{I}\right)^{2}-\eta_{Q}^{2}-\eta_{U}^{2}-\eta_{V}^{2}} \\
V= & -B_{v_{o}} \beta_{o} \mu \frac{\eta_{V}}{\left(1+\eta_{I}\right)^{2}-\eta_{Q}^{2}-\eta_{U}^{2}-\eta_{V}^{2}}
\end{array}
$$

mit $\mu=\cos \theta$, wobei $\theta$ den Winkel zwischen der Sichtlinie und der Normalen zur Sonnenoberfläche beschreibt ${ }^{1}$. Der Nenner dieser Gleichungen ist verantwortlich für die Sättigung der Linie, ein anliegendes Magnetfeld vermindert die Sättigung. Für ein longitudinales Magnetfeld beispielsweise sind $\eta_{Q}=\eta_{U}=0$ und $\eta_{V}^{2}$ schwankt je nach Feldstärke und Linienaufspaltung zwischen $\eta_{V}^{2}=0$ für $\Delta \lambda_{H}=0$ und $\eta_{V}^{2}=\eta_{I}^{2}$ für komplette Aufspaltung. Dementsprechend verringert sich der die Liniensättigung beschreibende Nenner mit zunehmendem Magnetfeld von $\left(1+\eta_{I}\right)^{2} \mathrm{zu}\left(1+2 \eta_{I}\right)$.

\footnotetext{
${ }^{1}$ Die explizite Wellenlängenabhängigkeit steckt in den $\eta$ und $\rho$ und wird durch die Voigt-Funktion und die Faraday-
} Funktion beschrieben, deren Parameter sich für die jeweilige Spektrallinie aus den atomaren Parametern ergeben. 


\section{Beobachtungen}

Grundlage jeder experimentellen Sonnenphysikarbeit ist und bleibt “das Experiment", d.h. die Gewinnung qualitativ hochwertiger Rohdaten, aus denen nach der Verarbeitung neue Erkenntnisse hervorgehen können.

Für die Gewinnung der für diese Arbeit verwendeten Daten wird das Göttinger Fabry-PerotSpektropolarimeter am Vakuum-Turm-Teleskop (VTT) in Izaña, Teneriffa, genutzt. Das VTT ist eines der beiden deutschen Teleskope für Sonnenphysik am Observatorio del Teide.

Im Rahmen meiner Tätigkeit am VTT wurden auch einzelne Kampagnen in Parallelbeobachtung mit dem Gregory-Coudé-Teleskop (GCT), dem zweiten deutschen Sonnenteleskop am Observatorio del Teide, durchgeführt. Diese Daten finden in der vorliegenden Dissertation allerdings keine Verwendung. Im Winter 2001/2002 wurde das GCT demontiert, um dem neuen 1,5 m Teleskop GREGOR Platz zu bieten.

\subsection{Vakuum-Turm-Teleskop}

Nach ausführlichen Sitetests wurde das Vakuum-Turm-Teleskop 1987 in Izaña auf 2400 m Höhe in Sichtweite des Pico del Teide aufgebaut. Genau dieser Standort wurde ausgewählt, weil er den folgenden Ansprüchen an gute Beobachtungsbedingungen gerecht wird:

1. Der wichtigste Aspekt für günstige Konditionen ist gutes "Seeing", welches bei gleichmäßiger und stabiler Luftschichtung der Erdatmosphäre gegeben ist. Starke Gradienten und Turbulenzen in der Atmosphäre bewirken unterschiedliche und wechselhafte Brechungsindizes für einzelne Lichtstrahlen, so dass das beobachtete Objekt verzerrt erscheint und flimmert. Da sich die Temperatur des Meeres durch die Sonneneinstrahlung über den Tag weniger ändert als die des Landes, ist die Luftschichtung über dem Meer stabiler. Demnach befindet sich die optimale Lage eines Teleskopes auf einer Insel.

2. Eine Alternative, den Luftturbulenzen der Erdatmosphäre zu entgehen, besteht darin, das Teleskop in einer gewissen Höhe zu montieren, so dass nur ein kleinerer Teil der Atmosphäre vom Licht durchlaufen wird. Um dieses zu erreichen, wurde der Rand der Cañadas auf der Vulkaninsel Teneriffa in einer Höhe von $2400 \mathrm{~m}$ als Standort des Teleskopes ausgewählt. 


\section{Beobachtungen}

3. Ein weiterer Vorzug des Standortes Teneriffa ist die ausgezeichnete geographische Lage der Insel. Teneriffa liegt auf $16^{\circ} 30^{\prime} 35^{\prime \prime}$ geographischer Länge und $28^{\circ} 18^{\prime} 00^{\prime \prime}$ geographischer Breite im Bereich des Passatwindes, der feuchte Luft gegen die Insel schiebt, so dass sich in $2000 \mathrm{~m}$ Höhe eine relativ stabile Wolkendecke und Inversionsschicht bildet: Am Fuße des Berges liegt eine kühle Luftschicht. Angewärmte Luft steigt hoch, bis sie auf $2000 \mathrm{~m}$ Höhe von den Wolken aufgehalten wird, über denen die Temperatur wieder abnimmt. Der Vorteil der Inversionswetterlage liegt darin, dass sich oberhalb der Wolken eine stabile Luftschichtung einstellen kann.

Das Vakuum-Turm-Teleskop ist ein Schiefspiegler mit Coelostatensystem und einer Brennweite von $46 \mathrm{~m}$. Bei diesem Teleskoptyp wird das Licht der Sonne durch zwei plane, drehbare Coelostatenspiegel vertikal auf den Hauptspiegel reflektiert. Die Rotationsachse des ersten Coelostaten, die Stundenachse, ist parallel zur Erdachse montiert, so dass durch Drehung um nur eine Achse die Erdrotation kompensiert werden kann. Der erste Spiegel reflektiert das Sonnenlicht auf den zweiten Spiegel, der es senkrecht durch das Haupteintrittsfenster in den Vakuumtank und auf den Hauptspiegel leitet.

Der Hauptspiegel des VTT ist ein leicht asphärischer Spiegel von $70 \mathrm{~cm}$ Durchmesser, der um einige Grad gegen die Senkrechte geneigt ist, so dass der Strahlengang seitlich gefaltet werden kann. Zu den Einrichtungen des Teleskopes gehört ein Correlation Tracker, der kurz vor dem Primärfokus installiert ist und durch Einfahren zweier Spiegel in Betrieb genommen werden kann. Aufgrund der Störanfälligkeit dieses Instrumentes wird auf die Benutzung jedoch verzichtet. Direkt einen Meter vor dem Primärfokus, im Hauptbeobachtungsraum (HBR, vgl. Abb. 3.5), befindet sich der um die Vertikale rotierbare $45^{\circ}$-Umlenkspiegel, der das Licht je nach Einstellung in eines der Optiklabore der verschiedenen Forschungsgruppen reflektiert.

\subsection{Göttinger Spektropolarimeter}

Die vorwiegende Arbeitsstätte der Göttinger Sonnephysikgruppe im VTT ist das Optiklabor 2 (OL 2) auf der Ostseite des Gebäudes. Hier befindet sich das 1990 von Cornelia Bendlin (1993) und Reiner Volkmer (1995) installierte Spektropolarimeter, das mit Weiterentwicklungen und Änderungen, vgl. Koschinsky (2001), im Kern noch besteht.

Die Aufgabe dieses Instrumentes ist die Gewinnung zweidimensionaler, räumlich sehr hochaufgelöster Spektren. Hierfür werden sehr schmalbandige Filtergramme in unterschiedlichen Wellenlängen benötigt, die mit Hilfe eines Fabry-Perot-Interferometers, s. Kap. 3.2.1, mit einer Halbwertsbreite von $44 \mathrm{~m} \AA$ (bei einem Plattenabstand von 1,5mm, einer Finesse von 30 und einer Wellenlänge von 6302,5 $\AA$ ) erstellt werden. Die Untersuchung von Magnetfeldern erfordert die Benutzung eines Polarimeters. Das in diesem Aufbau für die Trennung von rechtsund linkszirkular polarisiertem Licht benutzte Stokes- $V$-Polarimeter wird in Kap. 3.2.2 erläutert. Abschnitt 3.2.3 widmet sich schließlich der Beschreibung des aufwendigen Strahlenganges des Instrumentes. 


\subsubsection{Fabry-Perot-Interferometer}

Die wichtigste Eigenschaft von Fabry-Perot-Interferometern (FPIs) ist ihre wellenlängenabhängige Transmission von Lichtstrahlen.

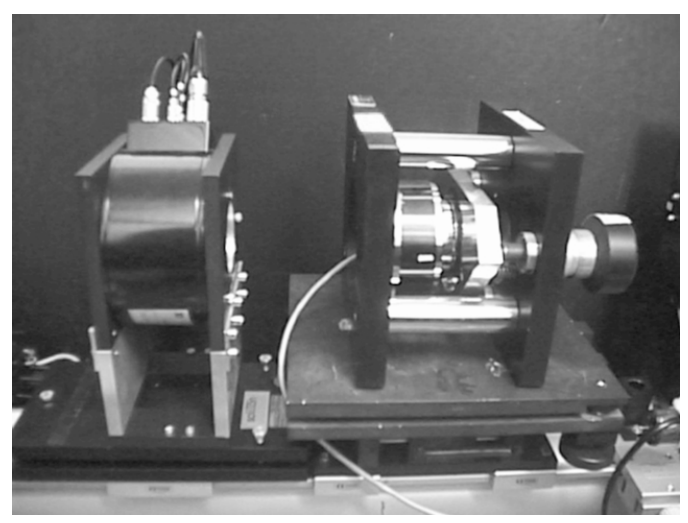

Abbildung 3.1: Photo der beiden Fabry-Perot-Interferometer des Göttinger Aufbaus.

Ein FPI, s. Abb. 3.1, besteht aus zwei parallelen, teilverspiegelten, leicht keilförmigen (Winkel zwischen 10 und 30 Bogenminuten) Glasplatten, die entgegengesetzt zueinander montiert sind. Die Glasplatten schließen eine planparallele Luftschicht der Dicke $d$ ein, in der die Lichtstrahlen, wie in Abb. 3.2 gezeigt, von den teilverspiegelten Glasplatten immer wieder reflektiert werden. Bei jeder Reflexion wird auch ein Teil des Lichtes durch die zweite Glasplatte entlassen, der größte Anteil aber wird reflektiert. So teilt sich der Lichtstrahl durch das FPI in viele Einzelstrahlen auf, die unterschiedlich oft zwischen den Glasplatten reflektiert werden. Abhängig von dem Abstand der Glasplatten werden sich die Einzelstrahlen beim Verlassen des FPIs durch konstruktive oder destruktive Interferenz auslöschen oder verstärken. Die optimale Transmission erfolgt nur für die Strahlen, für die die Weglänge zwischen den Glasplatten, abhängig von deren Abstand, gerade ganzzahligen Vielfachen ihrer Wellenlänge entspricht.
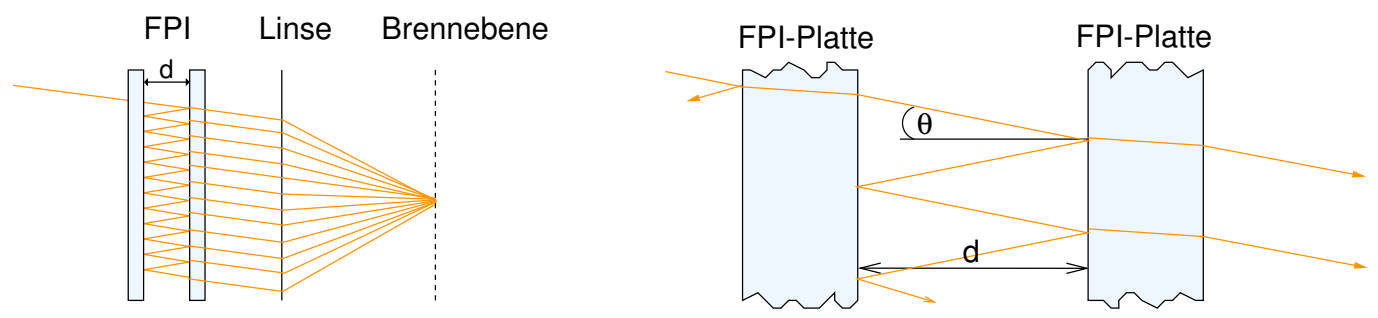

Abbildung 3.2: Schematische Darstellung der Funktion eines Fabry-Perot-Interferometers.

Der Transmissionsgrad $\frac{I_{t}}{I_{i}}$ eines Strahles, das Verhältnis des transmittierten Lichtes $I_{t}$ zum einfallenden Licht $I_{i}$, hängt neben der Wellenlänge $\lambda$ auch von der Reflektivität $F$ der teilverspiegelten 


\section{Beobachtungen}

Glasplatten des FPIs ab und wird (unter Vernachlässigung der Absorption) beschrieben durch die Airyfunktion:

$$
\frac{I_{t}}{I_{i}}(\lambda)=\frac{1}{F+\sin ^{2}\left(\frac{\delta(\lambda)}{2}\right)},
$$

wobei $\delta(\lambda)$ die Phasendifferenz zweier interferierender Strahlen darstellt:

$$
\delta(\lambda)=\frac{4 \pi}{\lambda} \cdot n d \cos \theta
$$

mit dem Brechungsindex $n$ der Luft zwischen den FPI-Platten, dem Abstand der Platten $d$ und dem Einfallswinkel zur Normalen $\theta$. Trägt man die Transmission gegen die Wellenlänge auf, ergibt sich die in Abb. 3.3 gepunktet dargestellte Airyfunktion:

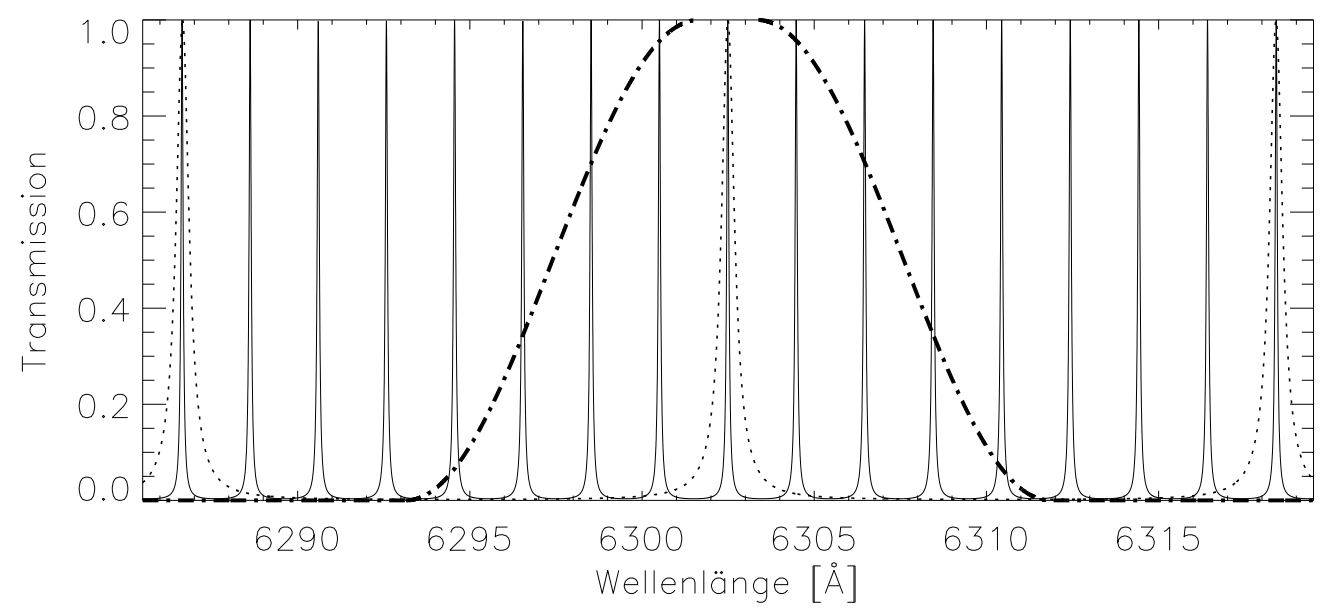

Abbildung 3.3: Airyfunktion der Fabry-Perot-Interferometer

durchgezogen: Airyfunktion des FPI2 (Finesse $\mathcal{F}_{\text {eff }, 2}=30, d=1 \mathrm{~mm}$ )

gepunktet: Airyfunktion des FPII (Finesse $\mathcal{F}_{\text {eff }, 1}=35, d=125 \mu \mathrm{m}$ )

strichpunktiert: Transmissionskurve des Interferenzfilters IF2 (schematisch, Halbwertsbreite $10 \AA$ )

In regelmäßigen Wellenlängenabständen zeigt die Abbildung ein Maximum der Transmission. Dieser Wellenlängenabstand wird der freie Spektralbereich (engl. free spectral range, FSR) genannt. Er hängt neben dem Plattenabstand $d$ des FPIs auch von der beobachteten Wellenlänge $\lambda$ ab:

$$
\mathrm{FSR}=\frac{\lambda^{2}}{2 d}
$$

Um sicher zu gehen, dass nur eine Ordnung des Lichtes das FPI passiert, muss ein Vorfilter eingefügt werden, dessen Breite so bemessen ist, dass sie nicht mehr als einen FSR durchlässt. Für diesen Zweck wird als "Vorfilter" ein zweites, breiteres Fabry-Perot-Interferometer benutzt. 
Dieses besitzt einen größeren FSR und wird kombiniert mit einem Interferenzfilter, das nur eine Ordnung des zweiten FPIs durchlässt. So entsteht ein Instrument, das in der Lage ist, sehr schmalbandige Filtergramme zu erzeugen.

Das spektrale Auflösungsvermögen des FPIs hängt von der Halbwertsbreite eines Durchlassbereiches $\Delta \lambda$ der Airyfunktion ab, weshalb als Maßstab für die Qualität des Auflösungsvermögens die Finesse $\mathcal{F}$ des FPIs als Quotient des FSR und der Halbwertsbreite defininiert wird:

$$
\mathcal{F}=\frac{\mathrm{FSR}}{\Delta \lambda}
$$

Die praktische Bestimmung der Finesse wird in Kap. 3.3.1 beschrieben.

\subsubsection{Stokes- $V$-Polarimeter}

Ein Stokes- $V$-Polarimeter besteht aus einer $\lambda / 4$-Phasenretardationsplatte und einem Polarimeter. Die $\lambda / 4$-Platte wandelt rechts- und links-zirkular polarisierte Lichtstrahlen in senkrecht zueinander linear polarisierte Lichtstrahlen um, während das Polarimeter, wie in Abb. 3.4 dargestellt, die polarisierten Strahlen räumlich trennt.

Kalkspatkristalle sind doppelbrechende Medien, in denen sich verschieden polarisierte Lichtstrahlen mit unterschiedlicher Lichtgeschwindigkeit ausbreiten, da sie andere Brechungsindizes erfahren. Diese Eigenschaft wird sowohl für die $\lambda / 4$-Platte als auch für das Polarimeter ausgenutzt.

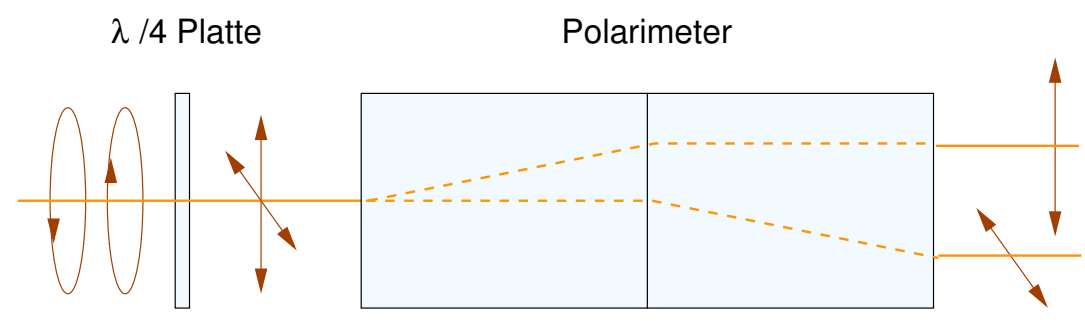

Abbildung 3.4: Strahlengang im Polarimeter.

Die $\lambda$ /4-Platte besteht aus einem sehr dünnen Kristall. Zirkular polarisiertes Licht verhält sich wie die Überlagerung zweier senkrecht zueinander stehender, linear polarisierter Wellen mit $90^{\circ}$ Phasenverschiebung, die wegen der unterschiedlichen Brechungsindizes für senkrecht zueinander stehende Strahlen verschieden lange optische Wege im Kristall zurücklegen. Die Dicke des Kristalles bzw. der Weglängenunterschied der beiden Strahlen ist so gewählt, dass die $90^{\circ}$ Phasenverschiebung der Komponenten des zirkular polarisierten Lichtes gerade ausgeglichen wird. Nach Verlassen des Kristalles schwingen beide Komponenten in Phase, das Licht ist linear polarisiert. Für zirkular polarisiertes Licht der anderen Richtung ergibt sich aus der Überlagerung der Komponenten linear polarisiertes Licht senkrecht zum ersteren. 


\section{Beobachtungen}

Auch das Polarimeter nutzt die doppelbrechenden Eigenschaften des Kalkspatkristalles. Zwei senkrecht zueinander linear polarisierte Lichtstrahlen erfahren im ersten Kristall durch die verschiedenen Brechungsindizes unterschiedliche Ablenkungen. Dieses wird beim Polarimeter ausgenutzt, um die beiden linear polarisierten Strahlen räumlich voneinander zu trennen. Die Länge des Kristalles wird hier so gewählt, dass die räumliche Distanz der Strahlen hinter dem Polarimeter der halben Breite der CCD-Detektorfläche entspricht, so dass die Bilder beider Strahlen nebeneinander auf dem Detektor abgebildet werden können. Da die optische Weglänge des ordentlichen Strahles kürzer ist als die des außerordentlichen Strahles, wird der Kristall halbiert und die zweite Hälfte um $90^{\circ}$ gedreht hinter die erste gesetzt. So setzt sich der Strahl, der den ersten Kristall als ordentlicher Strahl durchläuft, im zweiten Kristall als außerordentlicher Strahl fort und vice versa. Beide Strahlen durchlaufen so die gleiche optische Weglänge.

\subsubsection{Strahlengang}

Das Göttinger Spektro-Polarimeter ist ein komplexes Instrument, siehe Abb. 3.5 und 3.6, das fünf Teilstrahlengänge besitzt.

\section{Hauptstrahlengang}

Der $45^{\circ}$-Umlenkspiegel reflektiert das Sonnenlicht in die horizontale Ebene. Etwa einen Meter hinter dem Umlenkspiegel befindet sich im Primärfokus des Teleskopes ein Abbild der Sonne mit einem Durchmesser von $42 \mathrm{~cm}$. Eine runde Feldblende $F B 1$ wählt den Bildausschnitt aus und reduziert das Streulicht. Sie kann für die Justage durch einen Siemensstern oder ein feines Strichgitter ersetzt werden.

Der ausgewählte Bereich des Sonnenbildes wird durch zwei Transferlinsen TL1 und TL2 mit Brennweiten von $180 \mathrm{~cm}$ ins $O L 2$ transportiert und auf die Rechteck-Feldblende $F B 2$ abgebildet. Vor der FB2 befindet sich der erste Strahlteilerwürfel $B S 1$, der $5 \%$ des Lichtes für die BreitbandAufnahmen seitlich ablenkt (vgl. Breitbandstrahlengang).

Direkt hinter dem zweiten Fokus, in dem sich die Rechteckblende befindet, liegt das Stokes$V$-Polarimeter VP. Das Schmalband-Interferenzfilter IF2 mit einem Durchlassbereich von $10 \AA$ (für die Beobachtung in 6302,5 $\AA$ ) schließt sich an. Das Bild der Feldblende FB2 wird über die beiden Hauptlinsen $H L 1$ und $H L 2$ und, da das Ende des Raumes erreicht ist, einen Spiegel M3 auf die Kamera CCD2 abgebildet. Im parallelen Strahlengang zwischen HL1 und HL2 sind die beiden Fabry-Perot-Interferometer FPI1 und FPI2 montiert.

\section{Breitbandstrahlengang}

Für die Rekonstruktion der Schmalband-Daten ist es unerlässlich, exakt simultan mit den schmalbandigen auch breitbandige Bilder aufzunehmen. Aus diesem Grund wird vor der zweiten Feldblende FB2 mit Hilfe eines 5:95 Strahlteilerwürfels 5\% des Lichtes aus dem Hauptstrahlengang 


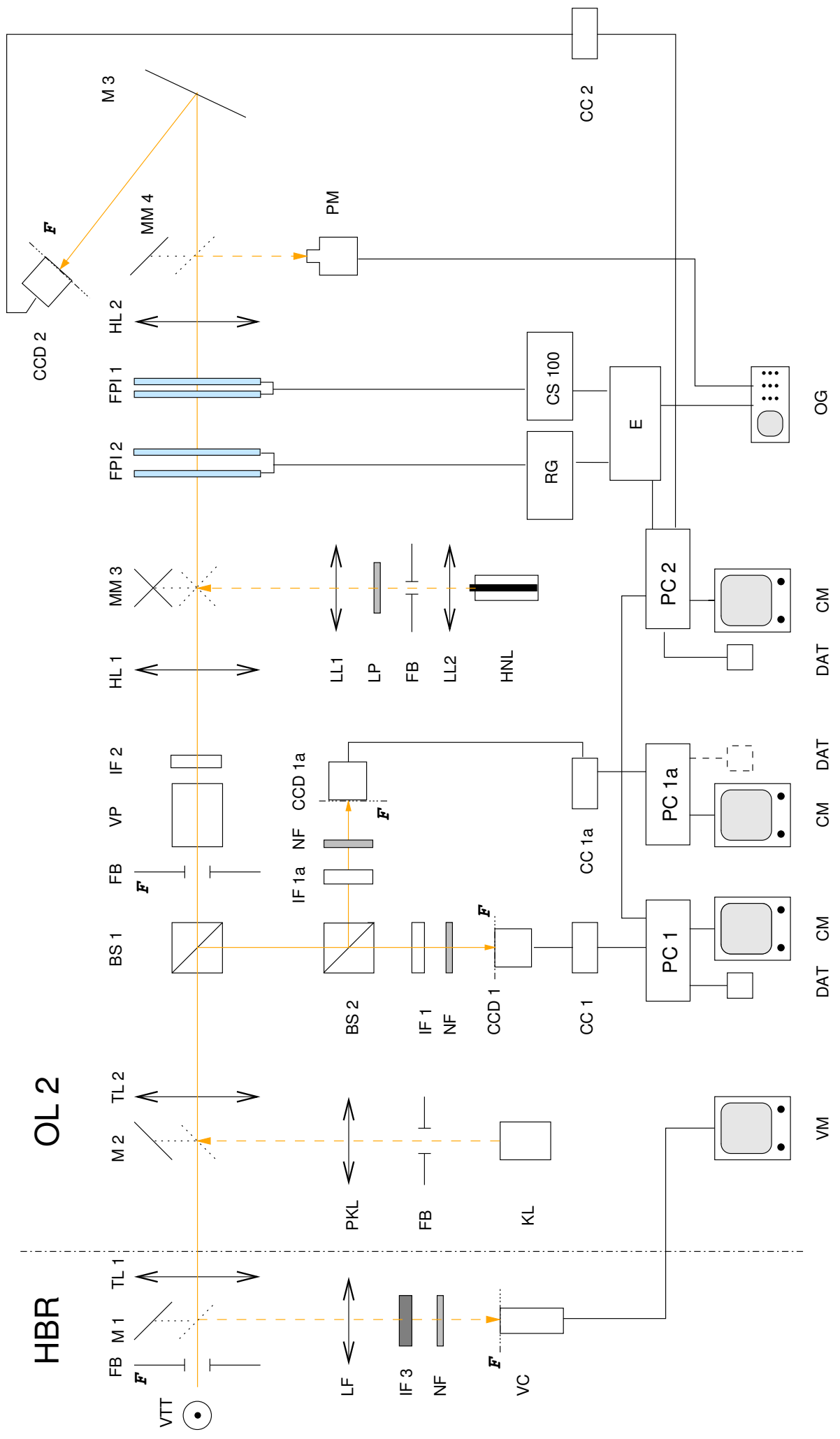

Abbildung 3.5: Schema des kompletten "Göttinger Aufbaus". Der Hauptstrahlengang verläuft von unten nach oben. 


\section{Beobachtungen}

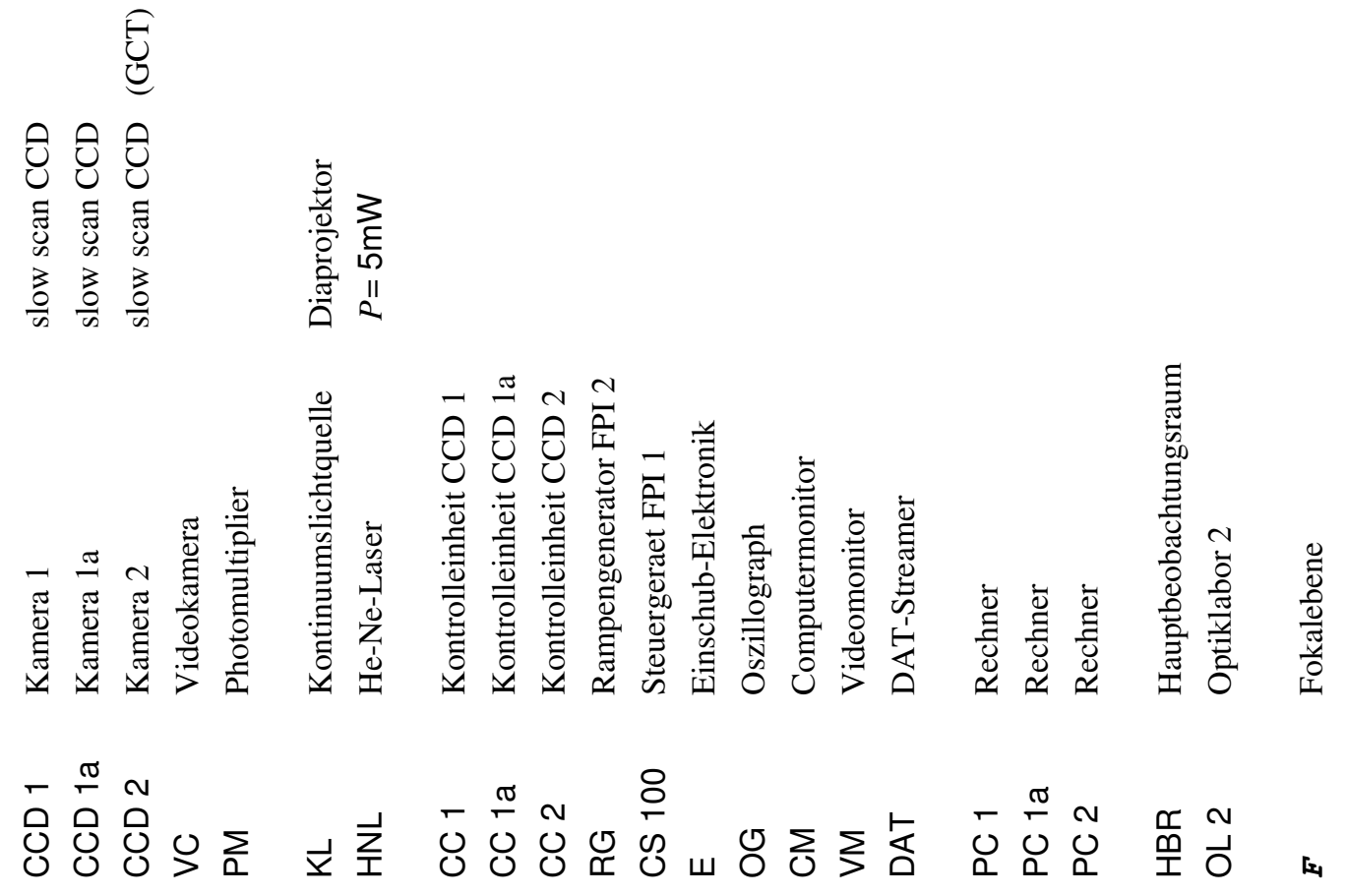

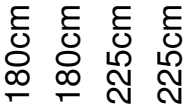

III II
है है

il

으으

ํㅛ ᄋ
हิ

?ำ

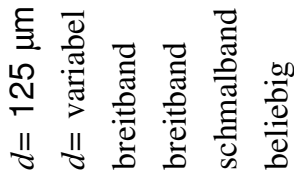

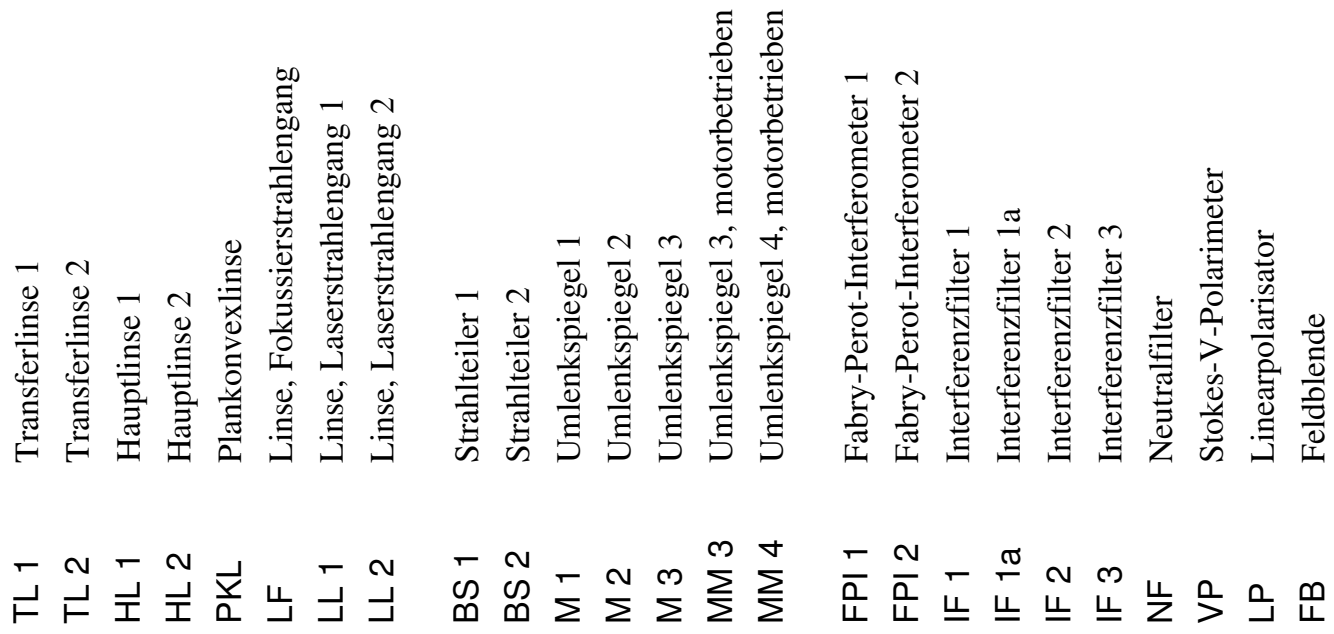

Abbildung 3.6: Legende zum Schema des "Göttinger Aufbaus" in Abb. 3.5. Die hier erläuterten Abkürzungen werden auch im weiteren Text ohne nochmalige Erklärung benutzt. 
ausgeblendet. Dieses Licht wird durch ein breitbandiges Interferenzfilter IF1 mit $100 \AA$ Halbwertsbreite und ein Neutralfilter $N F$ auf die Kamera $C C D 1$ fokussiert. Die beiden Steuercomputer $P C 1$ und $P C 2$ der Breitband- und Schmalbandkamera $C C D 1$ und $C C D 2$ sind miteinander verbunden, um sicherzustellen, dass die Belichtung der Kameras tatsächlich gleichzeitig geschieht.

Direkt hinter dem Strahlteiler BS1 kann optional ein weiterer Strahlteiler BS2 installiert werden, der $90 \%$ des Lichtes über ein Interferenzfilter IFI $a$ und ein Neutralfilter $N F$ auf der Kamera CCDla abbildet. Von dieser Möglichkeit wird Gebrauch gemacht, um G-Band Bilder der beobachteten Gebiete aufzuzeichnen. Da der für die Steuerung der CCDIa benutzte Computer PCla jedoch deutlich störanfälliger, langsamer und älter ist als $P C 1$ und $P C 2$, kann mit ihm nicht konsequent beobachtet werden.

\section{Kontinuumsstrahlengang}

Der Kontinuumsstrahlengang wird für die Korrektur von Intensitätsschwankungen beim Scan der FPIs aufgrund des Verlassens des optimalen Durchlassbereiches des IF2 benötigt. Hierfür befindet sich zwischen den Transferlinsen TL1 und TL2 ein Spiegel, der für diese Aufnahmen in den Strahlengang geschoben wird. Über diesen Spiegel wird anstelle des Sonnenlichtes das Licht einer Halogenlampe, die kontinuierliches Licht emittiert, in der Form eines Diaprojektors in den Strahlengang eingeführt. Die Fokussierung des Diaprojektors wird auf unendlich eingestellt, wobei diese Einstellung nicht wirklich paralleles Licht erzeugt, sondern vielmehr die Abbildung auf einen fernen Punkt verschiebt. Eine Plankonvexlinse $P K L$ erzeugt weit hinter dem Diaprojektor ein virtuelles Bild des gleichmäßig ausgeleuchteten Diaschachtes. Dieses extrem weit entfernte, virtuelle Bild wird durch TL2 auf die Fokalebene des Teleskopes abgebildet.

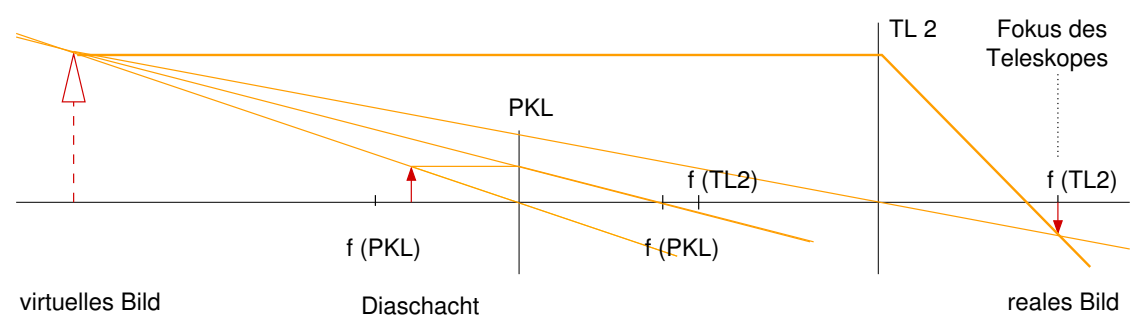

Abbildung 3.7: Schema des Lichtweges im Kontinuumsstrahlengang.

Die zweite Aufgabe der $P K L$ ist die Erzeugung eines virtuellen Bildes der Irisblende FB3, die zwischen Diaprojektor und $P K L$ installiert ist, im Brennpunkt der TL2. So bildet die TL2 die Irisblende, die in Größe und Form dem Pupillenbild des Teleskopes angepasst ist, auf den Ort des Pupillenbildes zwischen den FPIs ab. In der Fokalebene ist das Bild der Blende somit auf unendlich fokussiert.

Mit dem Einbau eines zweiten Fabry-Perot-Interferometers, das simultan mit dem ersten den beobachteten Spektralbereich abscannt, vgl. Kap. 3.2.1 und Kap. 3.3.2, ist die Benutzung des Kontinuumsstrahlenganges nicht mehr zwingend erforderlich. 


\section{Beobachtungen}

\section{Laser-Photomultiplier-Strahlengang}

Die exakte Parallelstellung der Glasplatten der FPIs, die Finesse (s. Kap. 3.3.1), kann unter Benutzung des Laser-Photomultiplier-Strahlenganges kontrolliert und korrigiert werden. Hierzu werden die zwei Spiegel MM3 und MM4 in den Hauptstrahlengang gefahren, die anstelle des Sonnenlichtes das Licht des Helium-Neon-Laser 11 HNL über die Linse $L L 1$ durch die beiden FPIs leiten. Nach Durchquerung der FPIs wird es von der HL2 auf den Photomultiplier PM fokussiert. Direkt hinter dem $H N L$ befindet sich eine Linse $L L 2$, die das Strahlenbündel des Lasers aufweitet, eine Feldblende $F B$ zur Streulichtreduzierung und ein Linearpolarisator $L P$ zur Intensitätsregulierung. Linse $L L 1$ erzeugt aus dem von $L L 2$ aufgeweiteten Lichtbündel wieder paralleles Licht.

\section{Video-Strahlengang}

Noch im Hauptbeobachtungsraum $H B R$, direkt hinter dem Primärfokus des Teleskopes, lässt sich ein weiterer Spiegel $M 1$ in den Strahlengang schieben, der das Sonnenlicht vom Teleskop auf eine Videokamera $V C$ umleitet. Mit einer Linse $L F$ wird das Bild der Fokalebene auf die Videokamera fokussiert. Zwischen $L F$ und $V C$ lassen sich Neutralfilter $N F$ und Interferenzfilter IF3 einsetzen.

Die Videokamera wird, da sie einen größeren Bildausschnitt als die CCDs zeigt, benutzt, um den beobachteten Bildbereich auszuwählen. Durch die Wahl eines G-Band-Interferenzfilters eignet sie sich besonders zum Auffinden von Fackelpunkten.

Das Bild der Videokamera wird direkt ins Optiklabor $O L 2$ übertragen und dient dort auch der Seeingkontrolle. Die Videokamera wird für die Zentrierung der Sonne und Fokussierung des Teleskopes benutzt. Sie muss daher unter Benutzung eines Siemenssternes gründlich fokussiert werden.

\subsection{Datengewinnung}

\subsubsection{Beobachtungspraxis}

\section{Vorbereitung}

Vor der Beobachtung muss das Herz des Teleskopes, der VME-Rechner, gebootet werden. Über das Terminal des VME-Rechners werden der zweite Coelostat und der Kreuztisch für die Nachführung in eine mittlere Position gebracht.

\footnotetext{
${ }^{1}$ Eine konstante Laserintensität ist für das Einstellen der Finesse von größtem Interesse. Die Intensität des Lasers springt direkt nach dem Einschalten um $\pm 20 \%$, nach einer längeren Laufzeit stabilisiert sie sich jedoch komplett. Der Helium-Neon-Laser wird daher während der kompletten Beobachtungskampagne ohne Unterbrechung betrieben.
} 
Die Stellung der beiden Coelostaten zueinander kann in gewissem Rahmen frei gewählt werden; so sind verschiedene Kombinationen von Winkeln der Coelostaten zueinander und der Säulenhöhe des zweiten Coelostaten möglich. Diese Einstellung ist aus mehreren Gründen wichtig:

- Die Reflexion des Lichtes an den Coelostaten zieht instrumentelle Polarisation nach sich, die gering gehalten werden soll.

- Die Säule des zweiten Coelostaten darf den ersten Coelostaten nicht abschatten.

- Bei Wind führt der zweite Coelostat in größerer Säulenhöhe beachtliche Schwingungen aus. In diesem Fall kann die nördliche Kuppelhälfte wieder halb geschlossen werden, um dem Coelostaten Windschatten zu bieten.

- Ein flacher Einfallswinkel des Lichtes auf den ersten Coelostaten bewirkt eine elliptische Apertur, die eine unterschiedliche Auflösung in den Bildachsen bewirkt; eine schlechte Voraussetzung für die Anwendung der Specklerekonstruktion.

Nach Wahl des Winkels und der Säulenhöhe wird der zweite Coelostat mit einem Handtaster in zwei Achsen so gekippt, dass der Lichtstrahl mittig auf den Hauptspiegel trifft.

\section{Finesse}

In Kap. 3.2.1 wurde die Funktionsweise eines FPIs erläutert und auch die Bedeutung der Finesse bereits erwähnt. Die Finesse beschreibt die Qualität des spektralen Auflösungsvermögens des FPIs, vgl. Formel 3.4, und hängt in der Praxis nicht nur von der Reflektivität der Spiegelplatten, sondern auch von eventuellen Unebenheiten oder einer möglichen Nichtparallelität der Platten ab. Die bei der Beobachtung gemessene Finesse wird daher effektive Finesse $\mathcal{F}_{\text {eff }}$ genannt.

Für ihre Einstellung wird ein Laserstrahl durch die FPIs gelenkt, wobei der Controller des zu korrigierenden FPIs eine Sägezahnspannung anlegt. Der Plattenabstand des FPIs ändert sich dadurch linear, so dass durch die Transmissionswellenlänge gescannt wird. Die Länge der Rampe wird so gewählt, dass gerade zwei Ordnungen des Laserlichtes zu sehen sind. Durch Änderung der Spannung der drei Piezoelemente der FPI-Platten wird nun die Parallelität der Platten korrigiert. Die Korrektur wird durch die Maximierung der Transmissionspeaks auf einem Oszilloskop kontrolliert. Beim neueren, breitbandigen FPI wird so eine effektive Finesse von 40 gemessen, die effektive Finesse des älteren, schmalbandigen FPI erreicht Werte um 35.

\section{Schrittweite des FPIs}

Die spektrale Schrittweite des FPIs während eines Scans sollte nach dem Abtast-Theorem kleiner gleich der halben Halbwertsbreite eines Durchlassbereiches $\frac{\Delta \lambda}{2}$ sein. Die Halbwertsbreite eines Durchlassbereiches ergibt sich nach Formel 3.4 aus der experimentell bestimmten, effektiven 


\section{Beobachtungen}

Finesse $\mathcal{F}_{\text {eff }}$ und dem freien Spektralbereich FSR. Der FSR wiederum ist mit Formel 3.3 gegeben durch die beobachtete Wellenlänge $\lambda$ und den Plattenabstand $d$ des schmalbandigen FPIs.

Hieraus ergibt sich für die benutzte Wellenlänge von 6302,5 $\AA$, siehe Kap. 3.4.1, bei einem Plattenabstand von 1,5 mm der freie Spektralbereich zu 1,324 A. Mit einer effektiven Finesse von 30 folgt hieraus eine theoretische Schrittweite von $22 \mathrm{~m} \AA$.

Die maximale Zahl der Aufnahmen pro Scan liegt technisch bedingt bei 150, so dass sich aus dieser Anzahl und der Gesamtbreite des zu scannenden Spektralbereiches zusammen mit der theoretischen Schrittweite von $\frac{\Delta \lambda}{2}$ die praktische Schrittweite ergibt. Hierbei muss abgewogen werden, wie viele Bilder pro Position und wie viele Positionen bei welcher Schrittweite möglich sind. Nach Festlegung von Anzahl der Wellenlängenpositionen und spektraler Schrittweite wird selbige für den Controller in AD-Schritte umgerechnet:

Eine Änderung der Transmissionswellenlänge um den FSR entspricht einer Änderung des Plattenabstandes von $\frac{\lambda}{2}$, für diese Plattenabstandsänderung wird gerade die nächste Ordnung durchgelassen. Ein AD-Schritt (ADS) der Steuerung bewirkt eine Änderung des Plattenabstandes um $2,52 \cdot 10^{-10} \mathrm{~m}$. Daher gilt

$$
\frac{\frac{\lambda}{2}}{2,52 \AA} \mathrm{ADS}=1 \mathrm{FSR}
$$

für den konkreten Fall mit $\lambda=6302,5 \AA$ und FSR $=1,324 \AA$ folgt hieraus $1 \mathrm{ADS}=1,05 \mathrm{~m} \AA$, für $\lambda=6149,2 \AA$ und FSR $=1,260 \AA$ ist $1 \mathrm{ADS}=1,03 \mathrm{~m} \AA$. Für die im Konkreten benutzten Einstellungen siehe Kap. 3.4 .1 .

\section{Kippung des Filters}

Die Wellenlänge, in der beobachtet wird, soll das Interferenzfilter im Schmalbandstrahlengang mit maximaler Transmission passieren. Um dieses zu gewährleisten, wird das Interferenzfilter leicht gegen die optische Achse gekippt.

Zunächst wird hierfür durch Scannen des Wellenlängenbereiches mit Rampe und Offset des FPII unter Zuhilfenahme eines Spektralatlanten die betreffende Linie identifiziert. Das Interferenzfilter muss sehr fein gekippt werden, bis die Intensität der Spektrallinie auf dem Oszilloskop ein Maximum erreicht. Hierfür ist es unerlässlich, dass die Finessen der beiden FPIs gut eingestellt worden sind.

Für das Interferenzfilter im breitbandigen Strahlengang ist diese Kippung nicht notwendig, da hier nicht die Transmission einer konkreten Wellenlänge sondern eines Wellenlängenbereiches im Vordergrund steht und für die Intensitäten eher umgekehrte Verhältnisse vorliegen: Obwohl für diese Bilder nur 5\% des Gesamtlichtes aus dem Hauptstrahlengang herausgespiegelt werden, benötigt man zur Reduktion der Intensität zusätzliche Graufilter, um den Höchstwert der Kamera von 4096 Counts nicht zu überschreiten. Diese Vorgabe sollte mit relativ langer Belichtungszeit in der Sonnenmitte überprüft werden, am frühen Vormittag unter Berücksichtigung eines zusätzlichen Sicherheitsfaktors von 4, damit die Aufzeichnung von Flatfields am Mittag nicht durch Überbelichtung verhindert wird. 


\section{Kippung des FPls}

Aus einem anderen Grund wird neben dem Interferenzfilter auch eines der beiden Fabry-PerotInterferometer gegen die optische Achse gekippt:

Zwischen FPI1 und FPI2 bilden sich Reflexionen, die nach Durchquerung des hinteren FPI auch auf die CCD2 abgebildet werden. Diese Reflexionen überlagern das Originalbild und verschmieren es, deshalb werden sie durch Schrägstellung des hinteren FPIs neben der CCD-Kamera abgebildet. Da eine Schrägstellung des FPIs mit Wellenlängenverschiebungen der Transmissionswellenlänge einhergeht, vgl. Kap. 3.4.2, sollte der Winkel des FPIs gegen die optische Achse so gering wie möglich gehalten werden. Im optimalen Fall liegt die erste Reflexion neben dem Hauptbild und berührt es gerade eben. Die Kippung des FPIs wird mit Hilfe des Monitors der CCD2 eingestellt, so dass die Reflexionen tatsächlich gerade außerhalb des Hauptbildes liegen.

\section{Fokussieren und Bildfeldabgleich}

Mit Hilfe eines Siemenssternes im Primärfokus des Teleskopes, eines scharfen Musters mit vom Zentrum ausgehenden hellen und dunklen Strahlen, werden CCD1 und CCD2, soweit für die Beobachtung eingeplant auch CCD1a, mit Kontrolle am Monitor fokussiert.

Nach erfolgreicher Fokussierung müssen die Bildfelder der Kameras aufeinander abgestimmt werden. Hierzu wird der Siemensstern durch ein Strichgitter ersetzt, das mit einer markanten Stelle auf die Mittellinien der Kameras abgebildet wird. Der Bildfeldabgleich wird dabei nicht nur durch horizontale und vertikale Verschiebung der Kameras, sondern auch durch Rotation um die optische Achse vorgenommen.

\section{Rechteckblende}

Da alle Aufnahmen der vorliegenden Arbeit unter Zuhilfenahme eines Polarimeters entstanden sind, besteht das Bild der Kamera CCD2 aus den zwei Teilbildern rechts- und links-zirkular polarisierten Lichtes. Bei der Einstellung der Rechteckblende, die den Bildausschnitt auf CCD2 festlegt und Streulicht zurückhält, werden somit nicht nur die Ränder des belichteten Bildfeldes und der Kamera übereinander gelegt, sondern auch die Grenzen der Teilbilder innerhalb des Feldes möglichst mittig festgelegt.

\subsubsection{Großjustage}

Im Frühjahr 2000 wurde eine Großjustage des gesamten Göttinger Aufbaus im Optiklabor 2 durchgeführt, die hier kurz erwähnt werden soll. 


\section{Beobachtungen}

\section{Linsenpositionen}

Nach Beschriftung der alten Positionen aller optischen Elemente wurden alle Linsen entfernt und ihre Brennweiten durch Reflexion an einem Spiegel ausgemessen. Die gemessenen Brennweiten wurden notiert und der Strahlengang entsprechend erneut aufgebaut. Es zeigte sich, dass die zuvor jahrelang nicht kontrollierten Positionen noch sehr gut stimmen.

Auch die Höhen der Linsen über der optischen Bank wurden gründlich justiert, so dass ein gerader Strahlengang vom $45^{\circ}$-Umlenkspiegel durch die Linsen bis zur CCD2 möglich ist. Die Linsen wurden mit dem Verlauf des Lichtes nacheinander eingesetzt und ausgerichtet, so dass der Lichtstrahl jede Linse mittig passiert.

Für die Fokussierung des Kontinuumsstrahlenganges steht ein Dia mit eingeritztem Fadenkreuz zur Verfügung, das durch die Plankonvex- und die Transferlinse scharf auf den bei der Rechteckblende liegenden Fokus abgebildet werden muss. Der Abstand zwischen der $P K L$ und der Irisblende, die das Pupillenbild zwischen den Transferlinsen TL1 und TL2 simuliert, wird ebenfalls kontrolliert.

\section{Gleichlauf der FPIs}

Auch der Gleichlauf der beiden Fabry-Perot-Interferometer wurde getestet. Hierzu benutzt man einen Spektralbereich ohne Absorptionslinien, so dass die Intensität über den gesamten Scanbereich gleich bleibt. Das schmalbandige FPI wird in der Flanke des breitbandigen FPIs positioniert und beide FPIs scannen in gleicher Geschwindigkeit die Wellenlänge des Kontinuumslichtes ab. Auf diese Weise ist schon eine kleine Wellenlängenabweichung der beiden FPIs als dramatische Erhöhung/Verringerung der Transmission auf der CCD2 oder dem Photomultiplier zu sehen. Es zeigt sich, dass FPI2 auf eine Distanz von 2,8 $\AA$ um $120 \mathrm{~m} \AA$ entsprechend $4 \%$ zu schnell fährt. Für einen Scan von $800 \mathrm{~m} \AA$ Gesamtscanbereich hätte dieses eine Gesamtabweichung der beiden FPIs von $34 \mathrm{~m} \AA$ bedeutet. Nach der Justierung des Gleichlaufes durch einen entsprechenden Korrekturfaktor in den Steuerungsprogrammen der Controller können die Geschwindigkeiten der FPIs so gut angepasst werden, dass auch für den größtmöglichen Scanbereich keine Abweichung mehr feststellbar ist. Der Fehler durch nicht exakten Gleichlauf der FPIs wird dadurch für die Beobachtungsscans praktisch auf Null reduziert.

\subsection{Datencharakteristika}

Mit dem frisch justierten Instrument konnten bereits im August 2000 und im Juni 2001 Daten besonders guter Qualität aufgezeichnet werden. Der teleskopabhängige Seeing-Parameter $\alpha$ der besten Scans reichte an Werte von 21 heran, entsprechend einem Fried-Parameter von $r_{0}=\alpha$. $D=15 \mathrm{~cm}\left(\right.$ mit $\left.D_{\mathrm{VTT}}=70 \mathrm{~cm}\right)$. 


\subsubsection{Organisation der Aufnahmen}

In insgesamt sieben Beobachtungskampagnen am VTT werden 69 Datenserien aufgezeichnet, wobei jede Serie aus 10 bis 20, selten auch 40 Scans besteht.

\section{Serien und Scans}

Ein Scan bezeichnet einen Datensatz von knapp 150 Schmalbandbildern, die sich auf verschiedene Wellenlängenpositionen aufteilen. Die Höchstgrenze von 150 Bildern pro Scan ist durch den begrenzten Speicherplatz (RAM) der benutzten Rechner bedingt, reicht jedoch aus, um eine Spektrallinie in 15 bis 30 Wellenlängenpositionen komplett abzuscannen. Die Anzahl der Wellenlängenpositionen hängt von der theoretischen Minimalschrittweite nach dem Abtast-Theorem und der Anzahl der gewünschten Bilder pro Position ab, vgl. Kap. 3.3. Letztere wiederum ist nicht ganz unabhängig von dem erwarteten Seeing, da für die erfolgreiche Rekonstruktion der Schmalbandbilder mindestens ein hochwertiges Bild pro Wellenlängenposition vorliegen sollte. Je nach Aufgabe werden verschiedene Kombinationen von Positionsanzahl, Bildern pro Position und Schrittweite benutzt. Für die Serie 11 mit dem besten Seeing, die im Folgenden noch häufiger zitiert werden soll, wurden 21 Positionen mit 7 Bildern pro Position benutzt. Die Gesamtbilderzahl liegt somit bei 147. Die Schrittweite zwischen zwei Positionen, vgl. Kap. 3.3. wird zu 28 ADS, entsprechend 29,4 mÅ gewählt, so dass der gesamte Scanbereich in der Wellenlänge 617,4 mÅ umfasst.

Die so aufgezeichneten Spektralscans sollen zu hochwertigeren Scans rekonstruiert werden, vgl. Kap. 4.2. Dazu ist es notwendig, dass exakt simultan zu den Schmalbandbildern eine gleiche Anzahl Breitbandbilder aufgezeichnet wird. Diese Breitbandbilder können in der Auswertung specklerekonstruiert werden, so dass die aus ihnen gewonnene Information über die Atmosphäre auf die Schmalbandbilder übertragen werden kann.

Für einige Serien werden zusätzlich zu den Breit- und Schmalbanddaten auch Scans im Licht des G-Bandes aufgezeichnet. Diese Scans dienen hauptsächlich als Orientierungshilfe und werden wegen technischer Schwierigkeiten mit dem die Kamera kontrollierenden Windows-Rechner nicht streng simultan zu den Schmalbanddaten gewonnen. Aufgrund einer längeren Abspeicherzeit liegt die maximale Bilderanzahl pro Scan hier bei 100 Bildern, zudem stürzt der Rechner regelmäßig nach drei bis fünf Scans ab, so dass keine kontinuierlichen Daten gewonnen werden können.

Alle Scans einer Serie zeigen die gleiche Spektrallinie in dem gleichen Bildausschnitt. Sie unterscheiden sich lediglich durch einen Zeitabstand von $49 \mathrm{~s}$ pro Scan und das sich ständig ändernde Seeing. Bei gutem Seeing können aus den Serien mit 10 bis 20 Scans daher kurze Zeitreihen von 8 bis 16 Minuten gewonnen werden, bei schlechtem Seeing werden 10 Scans pro Serie aufgezeichnet, um möglichst einen guten Scan zu erhalten. 


\section{Beobachtungen}

\section{Beobachtungsziele}

Da das ursprüngliche Ziel dieser Arbeit die Untersuchung der Mitte-Rand-Variation der Magnetfelder von Fackeln war, wurden hauptsächlich kürzere Serien mit verschiedenen $\cos \theta$ Positionen in der Nähe aktiver Gebiete der Sonne eingestellt. $\theta$ beschreibt hierbei den Winkelabstand des Sichtlinienstrahles zu der Senkrechten der Sonnenoberfläche, so dass durch $\cos \theta$ der Abstand zum Sonnenrand beschrieben werden kann. Die Bearbeitung dieses Themas gestaltete sich zunehmend schwierig, da auch oder gerade in den Serien höchster räumlicher Auflösung wenige bis gar keine magnetisch aktiven Fackelpunkte gefunden wurden.

Es entstanden auch drei Zeitreihen mit einer Länge von 40 Scans entsprechend 33 Minuten, da dieser Beobachtungspunkt allerdings nicht der obersten Priorität meiner Untersuchungen entsprach, wurden die Zeitreihen vorrangig in den Zeiten guten, jedoch nicht optimalen Seeings aufgezeichnet.

\section{Wellenlängen}

Der Schwerpunkt der Beobachtungen lag in der Wellenlänge der Linie des neutralen Eisens Fe I bei 6302,5 $\AA$, einige Beobachtungen wurden jedoch auch in der einfach ionisierten Fe II 6149,2 Linie durchgeführt. Beide Eisenlinien sind magnetisch sensitiv, die Fe I 6302,5 Å Linie mit einem Landé-Faktor von $\mathrm{g}=2,5$, die Linie bei $6149,2 \AA$ mit $\mathrm{g}=\frac{4}{3}$.

\section{Belichtungszeit}

Da für die korrekte Datenreduktion mit Specklerekonstruktion eine kurze Belichtungszeit der Einzelaufnahmen erforderlich ist, wird die Belichtungszeit so kurz wie möglich gewählt. Idealerweise sollte diese Zeit $20 \mathrm{~ms}$ nicht überschreiten. Leider ist die Einhaltung so kurzer Zeiten nicht immer möglich, da vor allem in den frühen Morgenstunden die Intensität der Sonne zu schwach ist. Zudem werden viele Beobachtungen am Rand der Sonne durchgeführt, so dass ein Kompromiss zwischen geringer Belichtungszeit und einer ausreichenden Anzahl an Counts für die schmalbandige CCD2 gefunden werden muss. Die gewählten Belichtungszeiten liegen somit in den meisten Fällen bei 30 ms oder sogar $40 \mathrm{~ms}$, womit 100 bis 300 Counts erreicht werden. Nur mit dem neuen Filter der Fe II 6149,2 Å Linie, das bei einer Halbwertsbreite von $7 \AA$ eine Transmission von $82 \%$ aufweist, werden auch mit 20 ms gute Ergebnisse erzielt.

\section{Bildgröße}

Alle Daten werden unter Benutzung des Stokes- $V$-Polarimeters gewonnen, so dass jedes Schmalbandbild aus zwei Teilbildern, respektive $\frac{1}{2}(I+V)$ und $\frac{1}{2}(I-V)$, besteht. Beide CCD Kameras besitzen $384 \times 286$ Pixel, wobei ein Pixel eine Breite von $0,105^{\prime \prime}$ entsprechend $75 \mathrm{~km}$ auf der Sonne abdeckt. Da die schmalbandigen Rohbilder demnach maximal $192 \times 286$ Pixel besitzen, müssen für die Rekonstruktion auch die größeren Breitbandbilder auf dieses Maß reduziert 
werden. Nach Abschluss der Rekonstruktion weisen die Bilder üblicherweise Maße von etwa $14^{\prime \prime} \times 24^{\prime \prime}$ oder $1.015 \times 1.740 \mathrm{~km}$ auf.

\subsubsection{Wellenlängenverschiebung}

Durch die Montierung der beiden Fabry-Perot-Interferometer im parallelen Strahlengang ergeben sich Wellenlängenverschiebungen, die in der Auswertung berücksichtigt werden müssen.

\section{Blauverschiebung über das Bildfeld}

Die Wellenlänge maximaler Transmission eines FPIs ändert sich mit dem Einfallswinkel des Lichtes. Wie in Abb. 3.8 illustriert ist, trifft das Licht jedes Bildpunktes unter einem anderen Winkel auf das FPI. Während das Licht des Bildpunktes, der auf der optischen Achse liegt (in Abb 3.8 der Einfachheit halber "Bildmittelpunkt" genannt), genau senkrecht in das FPI fällt, vergrößert sich dieser Winkel, je weiter der Bildpunkt sich von der optischen Achse entfernt.

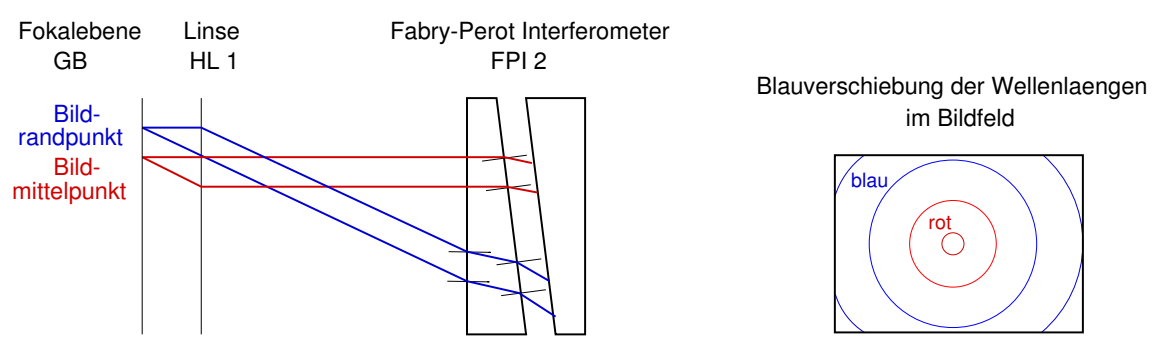

Abbildung 3.8: Blauverschiebung der Transmissionswellenlänge über das Bildfeld.

Betrachten wir zuerst den Bildpunkt auf der optischen Achse: Alle von diesem Punkt ausgehenden Strahlen verlaufen hinter der Linse parallel zur optischen Achse und treten senkrecht in die erste Glasplatte des FPIs ein. Beim Austritt dieser Lichtstrahlen aus der ersten FPI-Platte werden sie, da die Glasplatten des FPIs keilförmig sind, leicht gebrochen.

Vergleicht man den Weg, den diese Strahlen zwischen den FPI-Platten zurücklegen, mit dem Weg der Strahlen eines anderen Punktes, so sieht man gleich, dass der Weg für jeden anderen Bildpunkt ein längerer ist. Dies ist in Abb. 3.8 für den "Bildrandpunkt" genannten Punkt fern der optischen Achse verdeutlicht. Die Länge des Weges ist abhängig von dem Einfallswinkel des Strahles in die erste FPI-Platte. Da die maximale Transmissionswellenlänge gerade von der Weglänge der Strahlen zwischen den FPIs abhängt (vgl. Kap. 3.2.1), bedeutet dies, dass für jeden Bildpunkt eine andere Wellenlänge maximale Transmission besitzt. Die Abhängigkeit der Transmissionswellenlänge $\lambda_{t}$ von dem Einfallswinkel $\alpha$ des eintreffenden Strahles zur Normalen lässt sich ausdrücken durch

$$
\lambda_{t}=\lambda_{\perp} \cdot \cos \alpha
$$




\section{Beobachtungen}

Gegen die Transmissionswellenlänge des senkrecht auftreffenden Strahles treten also nur blauverschobene Wellenlängen auf, so dass dieses Phänomen "Blauverschiebung" über das Bildfeld genannt wird. Die Korrektur der Blauverschiebung wird in Kap. 4.3.6 beschrieben.

\section{Gradient über das Bildfeld}

Wie soeben erklärt, verändert der Winkel, unter dem ein Lichtstrahl auf das FPI trifft, die Wellenlänge der maximalen Transmission. Um die Überlagerung des bei der CCD2 aufgezeichneten Bildes mit den Reflexionen aus dem Zwischenraum der FPIs zu vermeiden, wird das FPI2 gegen die optische Achse gekippt (siehe Kap. 3.3.1). Diese Kippung zieht demnach, wie in Bild 3.9 erläutert, eine zweite Änderung der Transmissionswellenlänge nach sich.
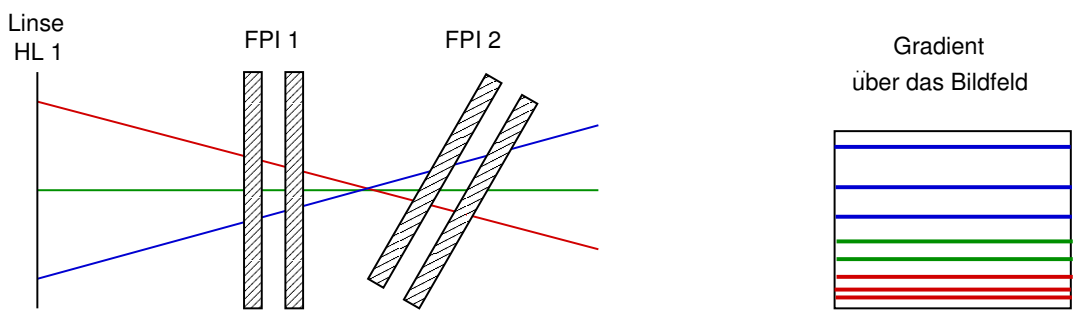

Abbildung 3.9: Entstehung eines eindimensionalen Gradienten über das Bildfeld.

Da die Kippung des FPIs um eine Achse senkrecht zur optischen Achse erfolgt, verändert sich die Wellenlänge für das Bildfeld nur in einer Raumrichtung. Eine Achse des Bildfeldes erfährt somit eine cosinusförmige Wellenlängenänderung, während die Konditionen der Punkte der anderen Achse sich gleichen. Diese Wellenlängenänderung wird in der Auswertung der Daten zusammen mit der Blauverschiebung korrigiert. 


\section{Datenreduktion}

Die am VTT mit Hilfe des Göttinger Spektropolarimeters gewonnenen Daten werden aufwendig aufbereitet, bevor die eigentliche Auswertung beginnt.

Zunächst wird aus den 147 Bildern des Breitbandkanales mit der Methode der Speckle-Rekonstruktion wie in Kap. 4.1 beschrieben ein hochaufgelöstes Breitbandbild erstellt. Die aus der Speckle-Rekonstruktion des Breitbandbildes gewonnenen Informationen über die Störungen der Atmosphäre werden benutzt, um auch die schmalbandigen Aufnahmen zu rekonstruieren, vgl. Kap. 4.2. Aus den 147 Bildern der verschiedenen Wellenlängenpositionen wird in den zwei Kanälen $\frac{1}{2}(I+V)$ und $\frac{1}{2}(I-V)$ pro Position und Kanal je ein Bild rekonstruiert. So ergibt sich für die Schmalbanddaten ein doppelter Datenkubus, der aus zwei Orts- und einer Wellenlängenachse besteht, siehe Abb.4.1.

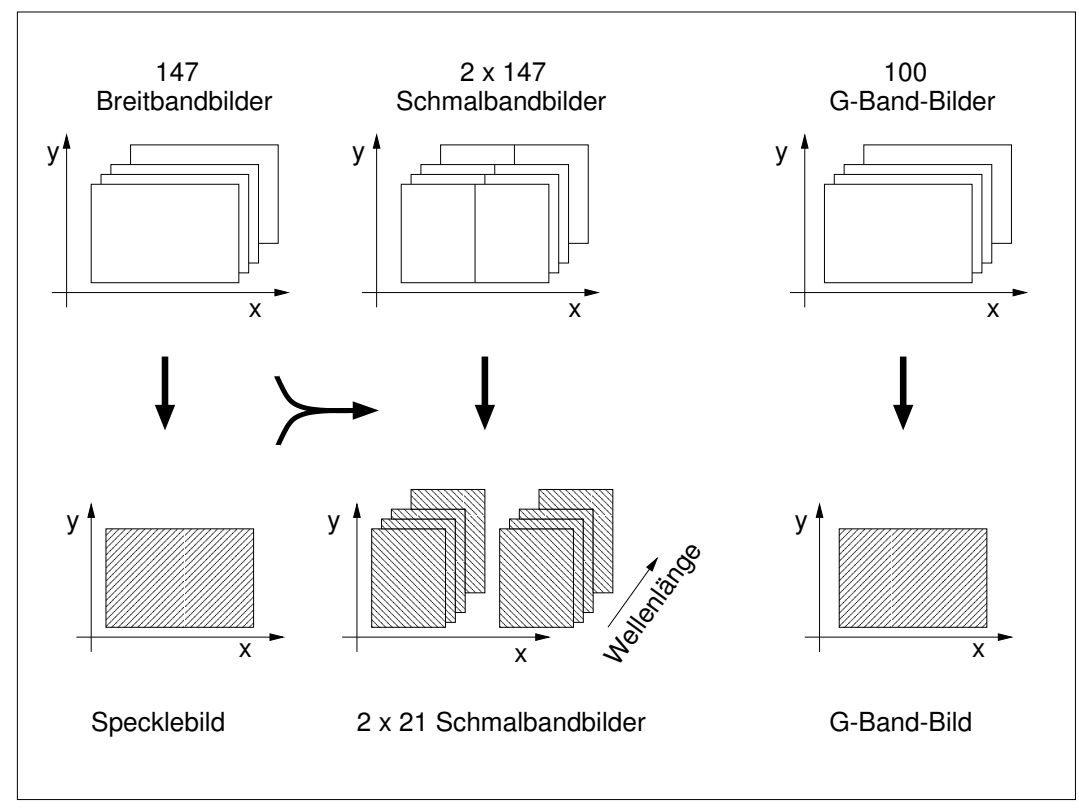

Abbildung 4.1: Organisation der Breit- und Schmalbanddaten.

Sofern G-Band Daten der CCD1a vorliegen, werden diese etwa 100 Bilder ebenfalls specklerekonstruiert, so dass hier aus jedem Scan ein qualitativ hochwertiges G-Bandbild resultiert. 


\subsection{Speckle-Rekonstruktion}

Die Speckle-Rekonstruktion ist eine Methode der Bildbearbeitung, bei der aus vielen, durch den Einfluss der Erdatmosphäre verzerrten Einzelbildern ein qualitativ hochwertiges Endbild berechnet wird. Voraussetzung hierfür sind eine große Anzahl Einzelbilder, die alle das identisch gleiche Objekt zeigen und sich nur durch den Einfluss der Atmosphäre unterscheiden. Im Folgenden soll das Prinzip dieser Methode kurz erläutert werden. Eine ausführliche Beschreibung der Speckle-Rekonstruktion findet sich bei de Boer (1993).

\section{Speckle-Transfer-Funktion}

Die von dem Objekt, der Sonne, kommenden Wellenfronten werden durch kleinskalige Temperaturschwankungen in der Erdatmosphäre verbogen, so dass nur ein verzerrtes Abbild des Objektes beobachtet werden kann. Diese kleinskalige Verzerrung ändert sich auf einer Zeitskala von etwa $10 \mathrm{~ms}$; über einen kleinen räumlichen Bereich von wenigen Bogensekunden kann sie als unabhängig von der Beobachtungsrichtung angenommen werden. Für diesen Bereich entspricht das beobachtete Abbild $i$ einer Faltung des wahren Objektes $o$ mit der Punktverbreiterungsfunktion $s$, alle drei abhängig von der Ortskoordinate $\vec{x}$ :

$$
i(\vec{x})=o(\vec{x}) \otimes s(\vec{x}) .
$$

Transformiert man diese Abhängigkeit in den Fourierraum, so ergibt sich die Fouriertransformierte des beobachteten Bildes $I(\vec{q}, t)$ aus einer einfachen Multiplikation der Fouriertransformierten des wahren Objektes $O(\vec{q})$ mit der optischen Transferfunktion $S(\vec{q}, t)$ :

$$
I(\vec{q}, t)=O(\vec{q}) \cdot S(\vec{q}, t),
$$

wobei $\vec{q}$ die Raumfrequenz und $t$ die Zeit darstellt. Wäre die optische Transferfunktion (OTF) bekannt, so könnte das wahre Objekt direkt berechnet werden.

Für lange Belichtungszeiten ist die optische Transferfunktion der Mittelwert vieler kurzzeitiger optischer Transferfunktionen. Die Langzeit-OTF $|\langle S(\vec{q}, t)\rangle|^{2}$ enthält keine Informationen über große Raumfrequenzen bzw. kleine Strukturen, weil diese weggemittelt werden.

Für kurze Belichtungszeiten von idealerweise weniger als $10 \mathrm{~ms}$ bleiben mehr Informationen über kleine Strukturen erhalten, denn die Kurzzeit-OTF fällt jenseits der Grenzfrequenz für Langzeitbelichtungen nicht direkt auf Null ab. Durch die Mittelung der quadrierten Amplituden der Kurzzeit-OTFs $\left\langle|S(\vec{q}, t)|^{2}\right\rangle$, Speckle Transferfunktion (STF) genannt, bleibt diese Information daher erhalten. Nach Labeyrie (1970) werden aus $n$ unabhängigen Aufnahmen die Betragsquadrate der Fouriertransformierten gemittelt und es gilt

$$
\left\langle|I(\vec{q}, t)|^{2}\right\rangle=|O(\vec{q})|^{2} \cdot\left\langle|S(\vec{q}, t)|^{2}\right\rangle
$$




\section{Spektraler Quotient}

Ziel ist nun die Bestimmmung der Speckle Transferfunktion $\left\langle|S(\vec{q}, t)|^{2}\right\rangle$. Handelt es sich bei dem Objekt um eine Punktlichtquelle, d.h. eine Deltafunktion im Ortsraum, so ist ihre Fouriertransformierte $|O(\vec{q})|^{2}$ konstant eins und die STF lässt sich direkt nach Formel 4.3 messen. Dies ist beispielsweise bei der Beobachtung eines einzelnen Sternes der Fall. Da es sich bei der Sonne aber um ein ausgedehntes Objekt handelt, kann die STF nicht direkt empirisch bestimmt werden.

Anstelle der Messung werden für die STF theoretische Modelle von Korff (1973) benutzt, die den Fried-Parameter $r_{0}$ als einzigen Parameter besitzen. Der Fried-Parameter ist ein Maß für die Qualität des Seeings und kann über die Methode des spektralen Quotienten nach von der Lühe (1984) bestimmt werden:

Es gilt

$$
\frac{|\langle I(\vec{q}, t)\rangle|^{2}}{\left\langle|I(\vec{q}, t)|^{2}\right\rangle}=\frac{|O(\vec{q})|^{2}}{|O(\vec{q})|^{2}} \cdot \frac{|\langle S(\vec{q}, t)\rangle|^{2}}{\left\langle|S(\vec{q}, t)|^{2}\right\rangle},
$$

wobei das Betragsquadrat der Fouriertransformierten des wahren Objektes $|O(\vec{q})|^{2}$ immer gleich bleibt, so dass es sich herauskürzen lässt. Das Verhältnis der beiden Mittelungen der beobachteten Aufnahmen wird spektraler Quotient $\varepsilon(\vec{q})$ genannt,

$$
\varepsilon(\vec{q}):=\frac{|\langle I(\vec{q}, t)\rangle|^{2}}{\left\langle|I(\vec{q}, t)|^{2}\right\rangle} .
$$

Der spektrale Quotient ist eine Funktion der Wellenzahl $\vec{q}$, dessen einziger Parameter ebenfalls der Fried-Parameter $r_{0}$ ist. Somit ist es möglich, aus dem spektralen Quotienten den FriedParameter $r_{0}$ zu bestimmen und anschliessend die STF mit Hilfe der theoretischen Modelle zu berechnen. Mit dieser STF werden nun nach Formel 4.3 die Amplituden des wahren Objektes rekonstruiert.

\section{Speckle Masking}

Die Amplituden des Objektes können mit der STF rekonstruiert werden, die Fourierphasen des wahren Objektes werden so jedoch nicht zurückgewonnen. Um das wahre Objekt vollständig rekonstruieren zu können, werden auch die Phasen der Fouriertransformierten, Fourierphasoren genannt, benötigt.

Die Fouriertransformierte des beobachteten Objektes lässt sich schreiben als $I(\vec{q}, t)=|I(\vec{q})|$. $e^{i \varphi(\vec{q}, t)}$. Da das Seeing die Phasen des fouriertransformierten Objektes willkürlich verändert und daher bei einer einfachen Mittelung alle Phasen $e^{i \varphi(\vec{q}, t)}$ gleich wahrscheinlich vorkommen, ergibt sich das Mittel zu Null. Die Methode von Knox \& Thompson (1974) mittelt daher anstelle der Phasen die Differenz je zweier Phasen. Sie benutzt dabei die Annahme, dass die Objektphasen 


\section{Datenreduktion}

zwar statistisch verteilt verfälscht werden, dass der Mittelwert der Phasendifferenz zweier Punkte aber unverändert bleibt.

In der Praxis wird eine Weiterentwicklung dieser Methode, das folgendermaßen definierte Speckle Masking Bispektrum nach Weigelt (1977), benutzt:

$$
I^{3}(\vec{q}, \vec{p}):=I(\vec{q}) \cdot I(\vec{p}) \cdot I^{*}(\vec{q}+\vec{p}),
$$

wobei * das komplex Konjugierte bezeichnet. Diese Methode besitzt eine verbesserte Zuverlässigkeit, da sie statt der einfachen Kreuzkorrelation des Knox-Thomson-Verfahrens eine Dreifachkorrelation verwendet. Die Bispektren aller Bilder werden gemittelt, entsprechend $\left\langle I^{3}(\vec{q}, \vec{p})\right\rangle$. Aus zwei bekannten Phasenwerten an den Punkten $\vec{q}$ und $\vec{p}$ kann mittels des Bispektrums die Phase an einem weiteren Punkt durch

$$
e^{\mathrm{i} \varphi(\vec{q}+\vec{p})}=e^{\mathrm{i} \varphi(\vec{q})} e^{\mathrm{i} \varphi(\vec{p})} e^{-\mathrm{i} \Phi(\vec{q}, \vec{p})}
$$

berechnet werden. Dies erlaubt die rekursive Berechnung des gesammten Phasenspektrums, wobei als Startwert die Phase der größten Strukturen verwendet wird da hier der Einfluss des Seeings minimal ist.

Die nun bekannten Phasen werden mit den zuvor bestimmten Amplituden zusammengefügt, so dass die komplette Fouriertransformierte des wahren Objektes bekannt ist und rücktransformiert werden kann.

\subsection{Rekonstruktion der Schmalbandbilder}

Nachdem aus den 147 Breitbandbildern ein hochwertiges Specklebild rekonstruiert wurde, wird dieses nach Keller \& von der Luehe (1992) folgendermaßen für die Rekonstruktion des Schmalbandbildes benutzt:

\section{Rekonstruktion}

Für ein isoplanares Gebiet gilt, dass sich das beobachtete Schmalbandbild $i_{n}$ wie das Breitbandbild $i_{b}$ aus einer Faltung der wahren Objekte $o_{n}$ bzw. $o_{b}$, die während der Aufnahmezeit unverändert bleiben, mit der Punktverbreiterungsfunktion (PSF) $s$ zusammensetzt: $i=o \otimes s$. Die PSF ist für beide Bilder identisch, sofern Breit- und Schmalbandbild im gleichen Wellenlängenbereich aufgezeichnet werden. Eine Faltung entspricht im Fourierraum einer Multiplikation, deshalb werden die Abbildungen fouriertransformiert betrachtet:

$$
I_{b}(\vec{q}, t)=O_{b}(\vec{q}) \cdot S(\vec{q}, t) \text { und } I_{n}(\vec{q}, t)=O_{n}(\vec{q}) \cdot S(\vec{q}, t)
$$

wobei $I$ die Fouriertransformierte des beobachteten, $O$ die Fouriertransformierte des wahren Bildes und $S$ die Fouriertransformierte der PSF, die optische Transferfunktion (OTF), beschreibt. 
Der Index $b$ bezeichnet breitbandige, $n$ schmalbandige Bilder, $\vec{q}$ bezeichnet die Frequenzkoordinate und $t$ die Zeit. Da sich die OTF für jedes einzelne Bild mit $\frac{I_{b}(\vec{q}, t)}{O_{b}(\vec{q})}=S(\vec{q}, t)$ aus der Rekonstruktion der Breitbandbilder bestimmen lässt, kann durch Anwenden der OTF auf jedes einzelne Schmalbandbild mit $S(\vec{q}, t)=\frac{I_{b}(\vec{q}, t)}{O_{b}(\vec{q})}=\frac{I_{n}(\vec{q}, t)}{O_{n}^{\prime}(\vec{q})}$ und durch Mittelung aller Einzelbilder einer Wellenlängenposition ein Schätzwert $O_{n}^{\prime}(\vec{q})$ für das wahre Objekt gefunden werden:

$$
O_{n}^{\prime}(\vec{q})=\left\langle\frac{I_{n}(\vec{q}, t)}{I_{b}(\vec{q}, t)}\right\rangle \cdot O_{b}(\vec{q})
$$

Da aber $S(\vec{q}, t)$ für einige Raumfrequenzen sehr kleine Werte annehmen kann, sind die einzelnen Quotienten in Formel 4.9 recht stark verrauscht. Ein besserer Schätzwert ergibt sich daher durch Gewichtung der Gleichung mit dem Powerspektrum des Breitbandbildes $\left|I_{b}(\vec{q}, t)\right|^{2}$ :

$$
O_{n}^{\prime}(\vec{q})=\frac{\left\langle I_{n}(\vec{q}, t) I_{b}(\vec{q}, t)^{*}\right\rangle}{\left\langle\left|I_{b}(\vec{q}, t)\right|^{2}\right\rangle} \cdot O_{b}(\vec{q})
$$

Die Notation $I^{*}$ bedeutet, dass es sich um die komplex Konjugierte des Bildes $I$ handelt. Durch Einsetzen von Formel 4.8 in 4.10 zeigt sich, dass tatsächlich $O_{n}^{\prime}(\vec{q})=O_{n}(\vec{q})$.

\section{Rekonstruktion verrauschter Daten}

Da die beobachteten Daten nicht nur aus dem Signal bestehen, sondern auch zufällig verteiltes Rauschen $N$ enthalten, muss für eine komplette Beschreibung jedoch

$$
I_{b}=O_{b} \cdot S+N_{b} \text { und } I_{n}=O_{n} \cdot S+N_{n}
$$

betrachtet werden. Eingesetzt in Formel 4.10 ergibt sich daraus

$$
O_{n}^{\prime}=\frac{O_{n} \overline{O_{b}}\left\langle|S|^{2}\right\rangle+O_{n}\left\langle S \overline{N_{b}}\right\rangle+\overline{O_{b}}\left\langle\bar{S} N_{n}\right\rangle+\left\langle\overline{N_{b}} N_{n}\right\rangle}{\left|O_{b}\right|^{2}\left\langle|S|^{2}\right\rangle+O_{b}\left\langle S \overline{N_{b}}\right\rangle+\overline{O_{b}}\left\langle\bar{S} N_{b}\right\rangle+\left\langle N_{b} \overline{N_{b}}\right\rangle} \cdot O_{b}
$$

Da das Rauschen des Breitbandkanales $N_{b}$ im Vergleich zu dem des Schmalbandkanales $N_{n}$ wesentlich kleiner ist, während $O_{b}$ und $O_{n}$ in der gleichen Größenordnung liegen, werden die $N_{b}$ enthaltenden Terme vernachlässigt. Wendet man nun die Rekonstruktion nicht auf ein Bild sondern auf ein nur Rauschen enthaltendes schmalbandiges Flatfield $N_{n, F F}$ an, so dass $O_{n, F F}=0$ und $O_{n, F F}^{\prime}=0+\varepsilon^{\prime}$, dann reduziert sich Formel $4.12 \mathrm{zu}$

$$
O_{n, F F}^{\prime}=\varepsilon^{\prime}=\frac{\overline{O_{b}}\left\langle\bar{S} N_{n, F F}\right\rangle+\left\langle\overline{N_{b}} N_{n, F F}\right\rangle}{\left|O_{b}\right|^{2}\left\langle|S|^{2}\right\rangle+\left\langle\left|N_{b}\right|^{2}\right\rangle} \cdot O_{b}
$$




\section{Datenreduktion}

und man erhält mit $\varepsilon^{\prime}$ eine Abschätzung des Rauschens.

Um die bestmögliche Abschätzung des Originalobjektes $O_{n}$ zu erhalten, wird das rekonstruierte Bild nach Brault \& White (1971) mit einem Optimumfilter $\Phi$ multipliziert, siehe Kap. 4.3.5, der gebildet wird aus

$$
\Phi=\frac{\left|O_{n}^{\prime}\right|^{2}-\left|\varepsilon^{\prime}\right|^{2}}{\left|O_{n}^{\prime}\right|^{2}}
$$

so dass sich die komplette Rekonstruktion ergibt zu

$$
O_{n}(\vec{q})=\Phi \cdot \frac{\sum_{t} I_{n}(\vec{q}, t) I_{b}(\vec{q}, t)^{*}}{\sum_{t}\left|I_{b}(\vec{q}, t)\right|^{2}} \cdot O_{b}(\vec{q})
$$

\subsection{Neuer CCD2-Programmcode}

Einen umfangreichen Teil der Arbeit zur Gewinnung hochaufgelöster Daten nimmt der Programmcode zur Rekonstruktion der Schmalbandbilder ein, der gründlich überarbeitet und an die polarimetrischen Daten angepasst wurde. Die Modifikationen des alten, nicht auf grosse Datenmengen ausgelegten Codes lassen sich in fünf Hauptpunkten zusammenfassen:

1. Beseitigung überflüssiger Schreibroutinen von Dateien, die später nicht mehr benutzt werden, und überflüssiger Leseroutinen von Variablen, die dem Programm bereits bekannt sind. Im Zuge dieser Änderung wurden auch Variablennamen, die doppelt benutzt waren, konsistent mit den Dateien neu eingerichtet.

Vorteil: Schnelligkeit der Programme

2. Zusammenfassung jeweils mehrerer Programme ähnlichen Inhaltes, wodurch einige Routinen nur einmalig ausgeführt werden und weitere Lese- und Schreibvorgänge obsolet werden.

Vorteil: Schnelligkeit der Programme

3. Änderung der Reihenfolge der Programme, so dass z.B. alle örtlichen Verschiebungen im gleichen Programm berechnet und auch ausgeführt werden. Ebenso werden die Wellenlängenverschiebungen in einem einzigen Programm berechnet und angewandt.

Vorteil: Übersichtlichkeit

4. Gründliche Überarbeitung der Programme, wobei gravierende Programmierfehler wie z.B. die falsche Blauverschiebung oder das falsche Flatfielding der Schmalbandbilder gefunden und behoben wurden. Auch inhaltliche Verbesserungen wie z.B. die neue Berechnung des Optimumfilters oder die neue Art der Flatfieldkorrektur werden eingefügt.

Vorteil: Bessere Qualität der Ergebnisse 
5. Adaption der Programme für polarimetrische Daten, wobei nur die Routinen doppelt durchlaufen werden, für die dieses notwendig ist. Hierbei wird strikt auf die Allgemeinheit geachtet, so dass die Programme ohne Nachteil auch für nicht polarimetrische Daten benutzbar sind und die fraglichen Programmteile in diesem Fall nur einmal durchlaufen werden. Vorteil: Schnelligkeit, Vereinfachung des Trackings

Die Auswirkungen dieser Überarbeitung sind gewaltig :

- Die Laufzeit der Programme konnte von 50 Minuten pro Scan auf unter 10 Minuten pro Scan gesenkt werden.

- Programme und Datenhandling sind wesentlich übersichtlicher.

- Störeffekte in den Ergebnisbildern konnten deutlich verringert werden.

- Die Ergebnisse sind "kompatibel" mit früheren Ergebnissen, jedoch von sichtbar besserer Qualität.

Im Folgenden soll die Datenreduktion der Schmalbandbilder etwas ausführlicher beschrieben werden.

\subsubsection{Vorbereitung der Daten}

Nach Abschluss der Beobachtungen werden die Daten auf DAT-Bänder überspielt und nach Göttingen transportiert. Sie werden auf den lokalen Rechnern eingelesen, wobei wegen der großen Datenmenge von etwa 10 GByte pro Beobachtungstag eine gute Organisation der Daten notwendig ist.

Für die Speckle-Rekonstruktion der Breitbandbilder wird die neueste Version des Programmpaketes von Pit Sütterlin auf der Basis der Programme von de Boer (1993) benutzt, das an der Universitäts-Sternwarte Göttingen geschrieben und weiterentwickelt wurde. Sofern keine unvorhergesehenen Probleme auftauchen, arbeitet dieses Programmpaket als Black Box und liefert das specklerekonstruierte Breitbandbild als Ergebnis.

Bevor die Daten in die Rekonstruktionsprogramme der Schmalbandbilder gegeben werden, müssen die beiden polarimetrischen Kanäle getrennt werden. Dazu werden die größtmöglichen Bereiche der linken und rechten Bildhälfte der CCD2, d.h. der beiden Kanäle $\frac{1}{2}(I+V)$ und $\frac{1}{2}(I-V)$, ausgeschnitten und anhand des kontrastreichsten Bildes aufeinander korreliert.

Abhängig vom konkreten Aufbau sind die CCD2 Daten gegenüber den CCD1 Daten um 180 Grad verdreht. Die CCD2 Daten werden nach den CCD1 Daten ausgerichtet abgespeichert. 


\section{Datenreduktion}

\subsubsection{Flatfield und Dark}

Um Empfindlichkeitsunterschiede der Kamerapixel ausgleichen zu können, wurde die Kamera im Laufe der Datengewinnung mit gleichmäßig hellen Bildern belichtet, Flatfield genannt. Durch Division der Daten durch die Flatfield Bilder wird die Empfindlichkeit der Pixel normiert.

Nicht nur die Empfindlichkeit, auch der Offset der Pixel unterscheidet sich. Durch Aufintegration in einer Kamera, die abgesehen von Streulicht nicht belichtet wird, werden diese Vorladungen der Pixel festgehalten. Diese Aufnahmen heissen Dunkelfeld oder Dark und werden noch vor der Flatfieldkorrektur von den Beobachtungen subtrahiert, wodurch alle vorhandenen Offsets auf Null gesetzt werden.

Die breitbandigen Aufnahmen der CCD1 werden dunkelkorrigiert und durch das auf Datenintensität normierte, mittlere Breitbandflatfield geteilt, so dass die Intensitätswerte aus der ruhigen Photosphäre nach der Division bei etwa eins liegen.

Auch die schmalbandigen CCD2 Daten werden zuerst dunkelkorrigiert. Da die schmalbandigen Daten als Scan in verschiedenen Wellenlängen aufgezeichnet werden, muss das Flatfield jeder dieser Wellenlängen getrennt bestimmt werden, denn für unterschiedliche Transmissionswellenlängen ändert sich der Plattenabstand der FPIs und somit der optische Weg. Zudem werden für die Korrektur der Schmalbanddaten zwei verschiedene Arten von Flatfield aufgezeichnet, fokussierte und defokussierte Flatfields.

Bei fokussierten Flatfields handelt es sich um Aufnahmen, während derer das fokussierte Teleskop zufällig, d.h. mit einem numerischen Zufallsgenerator gesteuert, sehr schnell in der Nähe der Sonnenscheibenmitte bewegt wird, so dass die Strukturen der Sonne stark verwischt sind. Durch Mittelung vieler dieser Bilder kann ein gleichmäßig belichtetes Bild ohne Struktur erstellt werden.

Die defokussierten Flatfields werden gewonnen, indem das Teleskop selbst defokussiert wird, so dass Strukturen der Sonne nicht mehr sichtbar sind. Die darkkorrigierten defokkussierten Flatfields werden pro Wellenlängenposition gemittelt. Sie werden in dem Rekonstruktionscode nur noch für die Berechnung des Optimumfilters, siehe Kap. 4.3.5, benutzt, da die Defokussierung des Teleskopes den Strahlengang stark abändert.

Die Flatfieldkorrektur der CCD2 Daten wird mit den fokussierten Flatfields ausgeführt, wofür die dunkelkorrigierten Flatfields einer Wellenlängenposition über sechs Scans mit je sieben Bildern pro Position gemittelt werden. Bevor die Schmalbanddaten wellenlängenpositionsweise durch die Flatfields dividiert werden, werden diese durch Multiplikation mit einem Faktor auf die Datenintensität normiert. Dieser Faktor wird durch Vergleich heller Pixel der Kontinuumspositionen des Datenscans und des Flatfieldscans gewonnen und soll den Mittelwert der Datenintensitäten für die Rekonstruktion auf eins setzen.

Dieses bedeutet, dass die schmalbandigen Daten nach der Korrektur des Dunkelfeldes durch die mittleren Positionsflatfields dividiert werden, ohne etwaige Wellenlängenverschiebung zwischen Flats und Daten zu beachten, siehe hierzu Kap.4.3.7. 
Alle Aktionen, die die schmalbandigen polarimetrischen Daten betreffen, werden jeweils für den linken und rechten Kanal getrennt ausgeführt. Es werden demnach zwei schmalbandige Darks berechnet, zwei mittlere, darkkorrigierte fokussierte Flatfieldscans und zwei mittlere darkkorrigierte defokussierte Flatfieldscans. Dieses gilt implizit auch für alle weiteren Schritte der Rekonstruktion, ohne dass es erneut erwähnt wird.

\subsubsection{Tracking}

Das Tracking korrigiert die örtliche Verschiebung der Einzelaufnahmen zueinander. Ein gutes räumliches Tracking ist für die Auswertung der Daten von großer Wichtigkeit, da alle Informationen, die aus den Scans gewonnen werden, nur dann sinnvoll sind, wenn die Helligkeitswerte der jeweils gleichen Strukturen miteinander verglichen werden.

Zunächst wird die Verschiebung der einzelnen Rohbilder gegeneinander (engl. Image Motion) korrigiert. Hierfür werden die breitbandigen CCD1 Rohbilder kontrastverstärkt und auf das kontrastreichste Rohbild korreliert. Die Verschiebevektoren, die für die Breitbandbilder gewonnen wurden, gelten ebenfalls für die kontrastärmeren Schmalbandbilder, wobei ein fester Offset zwischen Breitband- und Schmalbandbildern existiert. Dieser Offset zwischen CCD1 und CCD2 wird bestimmt, indem die für Image Motion korrigierten Breitband- und SchmalbandRohbilder der Wellenlängenpositionen des Kontinuums aufsummiert und aufeinander korreliert werden. Auch das specklerekonstruierte Breitbandbild wird auf das Kontinuumssummenbild der Breitband-Rohdaten korreliert, das auch für die Korrelation der Schmalbanddaten verwendet wurde.

Erst nach der Berechnung aller Verschiebungen inklusive des Image Motion mit dem CCD2CCD1 Shift für die schmalbandigen Rohdaten werden die Verschiebungen ausgeführt und neue Bilddimensionen berechnet, so dass die Verkleinerung der Rohbilder durch das Tracking minimiert wird.

\subsubsection{Rekonstruktion}

Nachdem alle Bilder den gleichen Bildausschnitt zeigen, werden die getrackten, geflatfieldeten Breitband- und Schmalbandrohbilder, das specklerekonstruierte Breitbandbild und auch das gemittelte defokussierte Flatfield in isoplanare Subbilder von $64 \times 64$ Pixeln Größe aufgeteilt, da die ankommenden Wellenfronten erfahrungsgemäß nur innerhalb eines solch kleinen Gebietes als isoplanar, d.h. in identischer Weise durch die Erdatmosphäre gestört, angesehen werden dürfen. Die Gebiete besitzen einen Überlappungsbereich von 30\%, damit sie nach der Rekonstruktion problemlos zusammengesetzt werden können. Ein 6 Pixel breiter Rand der Subbilder wird apodisiert, damit durch die periodisch arbeitende Fouriertransformation keine Fehler produziert werden. Vor der Rekonstruktion wird von jedem Subbild eine Regressionsfläche abgezogen, die nach der Rekonstruktion wieder aufaddiert wird. Dies ist zulässig, da Gradienten über das gesamte isoplanare Gebiet von den kleinskaligeren Schwankungen des Seeings nicht beeinflusst werden. 


\section{Datenreduktion}

Die Subbilder werden nun entsprechend Kap. 4.2 rekonstruiert: Jeweils alle räumlich gleich gelegenen Subbilder einer Wellenlängenposition von CCD1 und CCD2 und das räumlich gleich gelegene Specklesubbild werden gemeinsam verarbeitet.

Im Fourierraum wird das Produkt der Schmal- und Breitbandbilder aufsummiert und durch das Powerspektrum des Breitbandbildes dividiert, vgl. Formel 4.15. Die so vorbereiteten Bilder müssen vor ihrer Rückstransformation in den Ortsraum noch mit dem Optimumfilter multipliziert werden. Hierfür werden die schmalbandigen, defokussierten Flatfieldsubbilder in der gleichen Weise wie die Schmalbandsubbilder vorbereitet, so dass der Optimumfilter der Wellenlängenposition wie in Kap. 4.3.5 beschrieben konstruiert werden kann.

Nach der Anwendung des Optimumfilters wird das Subbild in den Ortsraum zurücktransformiert. Die Subbildpositionsmittel der zuvor subtrahierten Gradientenflächen werden auf die rekonstruierten Bilder addiert, die schliesslich zu einem rekonstruierten Gesamtbild pro Wellenlängenposition zusammengesetzt werden, wobei im Überlappungsbereich zweier Subbilder ein gewichteter Mittelwert benutzt wird.

\subsubsection{Optimumfilter}

Die Multiplikation des im Fourierraum vorverarbeiteten Subbildes mit einem Optimumfilter ist notwendig, da bei der Rekonstruktion in der Praxis auch kleinskalige Strukturen verstärkt werden, die unter der Auflösungsgrenze des Teleskopes liegen. Dieses Rauschen stört die Ergebnisbilder sichtbar, deshalb schneidet der Optimumfilter im Fourierraum alle Strukturen über einer gewissen Wellenzahl ab.

Zu Beginn dieser Arbeit wurden verschiedene starre Filter getestet, eine weit bessere Lösung jedoch ist die Anpassung eines Optimumfilters an das jeweilige Rauschen der Subbilder. Hierfür werden wie in Kap. 4.2 beschrieben die defokussierten Flatfieldsubbilder wie die Schmalbanddatensubbilder vorbereitet (vgl. Formel 4.13). Aus den vorbereiteten Flatfield- und Schmalbandsubbildern wird durch Multiplikation mit ihrem konjugiert Komplexen das Powerspektrum der Schmalband- und Flatfieldsubbilder erstellt.

Durch Mittelung dieser Powerspektren über alle Subbilder einer Wellenlängenposition und Vergleich nur der hohen Frequenzen wird ein Faktor gewonnen, mit dem das Flatfieldpowerspektrum auf das Datenpowerspektrum normiert wird. Als Schwellenwert für die hohen Frequenzen, bei denen sowohl Flat- als auch Datenpowerspektrum nur Rauschen enthalten, wird die Wellenzahl $k=25 \operatorname{~arcsec}^{-1}$ benutzt, da diese bei einer Bildgröße von 64 Pixeln die kleinsten Strukturen beschreibt, die theoretisch in den Daten enthalten sein könnten.

Der Optimumfilter wird nun durch Division des Datenpowers abzüglich des normierten Flatfieldpowers durch das unveränderte Datenpower gewonnen (vergleiche Kap.4.2), was dem Verhältnis

$$
\frac{\text { Signal }}{\text { Signal + Rauschen }}
$$


entspricht. Der Optimumfilter fällt so wie gewünscht mit abnehmendem Signal relativ zum Rauschen von eins auf null ab.

Da auch dieser Optimumfilter noch Rauschen beinhaltet, wird er geglättet. Durch kreisförmige Integration um den zuvor in den Mittlepunkt verschobenen Optimumfilter wird der Radius des Abfalls auf unter 0,5 bestimmt. Für diesen Radius wird ein künstlicher Filter mit einer FermiVerteilung erstellt, mit dem die schmalbandigen Subbilder nach der Rekonstruktion bearbeitet werden.

\subsubsection{Wellenlängenverschiebung}

Aufgrund des schrägen Durchgangs des Lichtes durch die beiden Fabry-Perot-Interferometer entsteht, wie in Kap. 3.4.2 erläutert, im Bild eine positionsabhängige Blauverschiebung der Wellenlängen maximaler Transmission. Für die Korrektur dieser Wellenlängenverschiebung werden die Wellenlängenprofile der mittleren $11 \times 11$ Pixel des mittleren fokussierten Flatfields gemittelt. Das so erstellte Referenzprofil wird als Grundlage der Verschiebungsberechnung benutzt.

Pixelweise wird jedes Flatfieldprofil auf das Referenzprofil korreliert und die Verschiebung für jeden Pixel in einer Matrix gespeichert. Diese Verschiebungsmatrix wird zweidimensional mit einem Polynom dritter Ordnung gefittet. Aus der Theorie hätte man die Überlagerung zweier Cosinusfunktionen erwartet. Da der Fit des Polynoms dritter Ordnung die tatsächlichen Verschiebungen jedoch sehr gut wiedergibt, wird dieser Fit benutzt.

Nach der Rekonstruktion wird die Verschiebungsmatrix auf alle Datenprofile angewandt, wofür eine Interpolation der Datenprofile erforderlich ist. Die Linienkernpositionen der Datenprofile stimmen nun mit der Linienkernposition des Referenzprofiles überein.

Die Wellenlängenverschiebung wird für den rechten und den linken Kanal getrennt berechnet, in beiden Fällen wird aber das aus dem linken Bildfeld gewonnene Referenzprofil für die Ermittlung der Verschiebungen zugrunde gelegt; ein eventueller Wellenlängenoffset zwischen der rechten und der linken Bildhälfte wird im gleichen Schritt mitkorrigiert.

\subsubsection{Unverbreitertes Flatfieldprofil}

Vor der Rekonstruktion der schmalbandigen Daten werden selbige flatfieldkorrigiert, indem sie durch das für die Kontinuumspositionen auf die Datenintensität normierte, über eine Wellenlängenposition gemittelte, fokussierte Flatfield dividiert werden. Durch diese Division wird die spektrale Information der Daten teilweise entfernt. Diese Information muss durch Multiplikation mit einem mittleren Flatfieldprofil wieder zurückgewonnen werden.

Die Flatfieldprofile werden hierfür analog den Datenprofilen interpoliert, um die Blauverschiebung entsprechend Kap. 4.3.6 zu korrigieren. Die Linienkernpositionen aller Flatfieldprofile liegen somit an einer Linienkernposition des Referenzprofiles, so dass keine relative Wellenlängenverschiebung zwischen Flat und Daten existiert. Diese Flatfieldprofile werden nun über das Bildfeld gemittelt, so dass ein unverbreitertes, rauscharmes Flatfieldprofil ensteht. 


\section{Datenreduktion}

Einerseits soll die spektrale Information an die Daten zurückgegeben werden, andererseits darf die eigentliche Aufgabe des Flatfields, die Korrektur der Pixelempfindlichkeit, nicht rückgängig gemacht werden. Deshalb werden die Datenprofile mit dem unverbreiterten Flatfieldprofil multipliziert.

Dieses Flatfield muss, wie auch vor der Rekonstruktion, in der Intensität wieder auf die der Daten normiert werden. Zusätzlich sollen die Datenprofile für die ruhige Photosphäre der Kontinuumspositionen auf eins normiert werden. Diese beiden Faktoren, $\frac{\text { Datenintensitaet }}{\text { Flatintensitaet }}$ und $\frac{1}{\text { Datenintensitaet }}$, werden zusammengefasst und entsprechen einer Normierung der Kontinuumspositionen des Flatfields auf eins. Die Normierungen werden weder pixelweise noch wellenlängenpositionsweise durchgeführt, es handelt sich hierbei lediglich um einen Faktor für den gesamten Scan. Dies ist korrekt, da weder einzelne Pixel noch einzelne Wellenlängenpositionen mit unterschiedlichen Faktoren behandelt werden dürfen. Nach Abschluss der Normierung sind auch die Linienprofile der Schmalbanddaten rekonstruiert.

\subsection{Magnetfeld}

Nach der Rekonstruktion der Schmalbandbilder liegt für jeden Pixel des zweidimensionalen Bildfeldes ein komplettes Wellenlängenprofil links- und rechtszirkular polarisierten Lichtes vor. Aus den zwei Datenkuben des $\frac{1}{2}(I+V)$ und $\frac{1}{2}(I-V)$ Kanales, wird zum einen durch die Amplitude des Stokes- $V$-Signales, vgl. Kap. 4.4.3, zum anderen durch die Linienschwerpunktsmethode, vgl. Kap. 4.4.4, das Magnetfeld der im Breitbandbild sichtbaren Strukturen bestimmt.

\subsubsection{Crosstalk}

Eine typische Fehlerquelle für die Auswertung von Stokes- $V$-Signalen ist der Crosstalk von Stokes- $I$ nach Stokes- $V$, der durch polarisierende Eigenschaften bei Reflexionen an Teleskopspiegeln zustande kommt. Auf diese Thematik soll deshalb kurz eingegangen werden.

Der Crosstalk, das Übersprechen der Stokes-Parameter (Kap. 2.3) aufeinander, wird von der Muellermatrix beschrieben, wobei sich die beobachteten Parameter aus einer Summe verschiedener Anteile der ursprünglich vorhandenen Parameter ergeben.

Die Multiplikation des Stokes-Vektors mit einer Muellermatrix sieht allgemein wie folgt aus,

$$
\left[\begin{array}{c}
I_{\lambda}^{\prime} \\
Q_{\lambda}^{\prime} \\
U_{\lambda}^{\prime} \\
V_{\lambda}^{\prime}
\end{array}\right]=\left[\begin{array}{llll}
\sigma_{11} & \sigma_{12} & \sigma_{13} & \sigma_{14} \\
\sigma_{21} & \sigma_{22} & \sigma_{23} & \sigma_{24} \\
\sigma_{31} & \sigma_{32} & \sigma_{33} & \sigma_{34} \\
\sigma_{41} & \sigma_{42} & \sigma_{43} & \sigma_{44}
\end{array}\right] \cdot\left[\begin{array}{c}
I_{\lambda} \\
Q_{\lambda} \\
U_{\lambda} \\
V_{\lambda}
\end{array}\right]
$$

so dass für den beobachteten Stokes-Parameter $V^{\prime}$ gilt 


$$
V_{\lambda}^{\prime}=\sigma_{41} I_{\lambda}+\sigma_{42} Q_{\lambda}+\sigma_{43} U_{\lambda}+\sigma_{44} V_{\lambda}
$$

Da in den vorliegenden, kleinskaligen Magnetfeldern erwartet werden kann, dass $Q \ll V$ und $U \ll V$, ist der Crosstalk $Q \rightarrow V$ und $U \rightarrow V$ vernachlässigbar (Koschinsky et al. 2001) und es bleibt

$$
V_{\lambda}^{\prime}=\sigma_{41} I_{\lambda}+\sigma_{44} V_{\lambda}
$$

Der Anteil von $V$ an $V^{\prime}$ beträgt nach Abschätzungen ähnlicher Teleskope etwa $90 \%$ (Capitani et al. 1989; Sanchez Almeida et al. 1991). Eine Möglichkeit zur Abschätzung des Crosstalk von $I \rightarrow V$ besteht darin, ausschließlich die Flatfields zu betrachten, da diese kein $V$-Signal enthalten sollten. Für die Flatfields gilt dann mit $V_{\lambda}=0$

$$
V_{\lambda}^{\prime}=\sigma_{41} I_{\lambda}
$$

wobei $V^{\prime}$ das beobachtete Stokes- $V$-Signal und $I$ die Gesamtintensität darstellt. Auf diese Weise kann $\sigma_{41}$ für jede einzelne Wellenlängenposition bestimmt werden. $V^{\prime}$ und $I$ sind wellenlängenabhängig, $\sigma_{41}$ dagegen ist eine über den betrachteten kleinen Wellenlängenbereich konstante Größe, die aus der instrumentellen Polarisation stammt. Mit $L_{\lambda}=\frac{1}{2}\left(I_{\lambda}+V_{\lambda}^{\prime}\right)$ und $R_{\lambda}=\frac{1}{2}\left(I_{\lambda}-V_{\lambda}^{\prime}\right)$ lässt sich der Crosstalk $I \rightarrow V$ nun berechnen durch

$$
\sigma_{41}=\frac{V_{\lambda}^{\prime}}{I_{\lambda}}=\frac{L_{\lambda}-R_{\lambda}}{L_{\lambda}+R_{\lambda}}
$$

Abb. 4.2 zeigt die Anwendung dieses Zusammenhangs auf die Beobachtungsdaten.

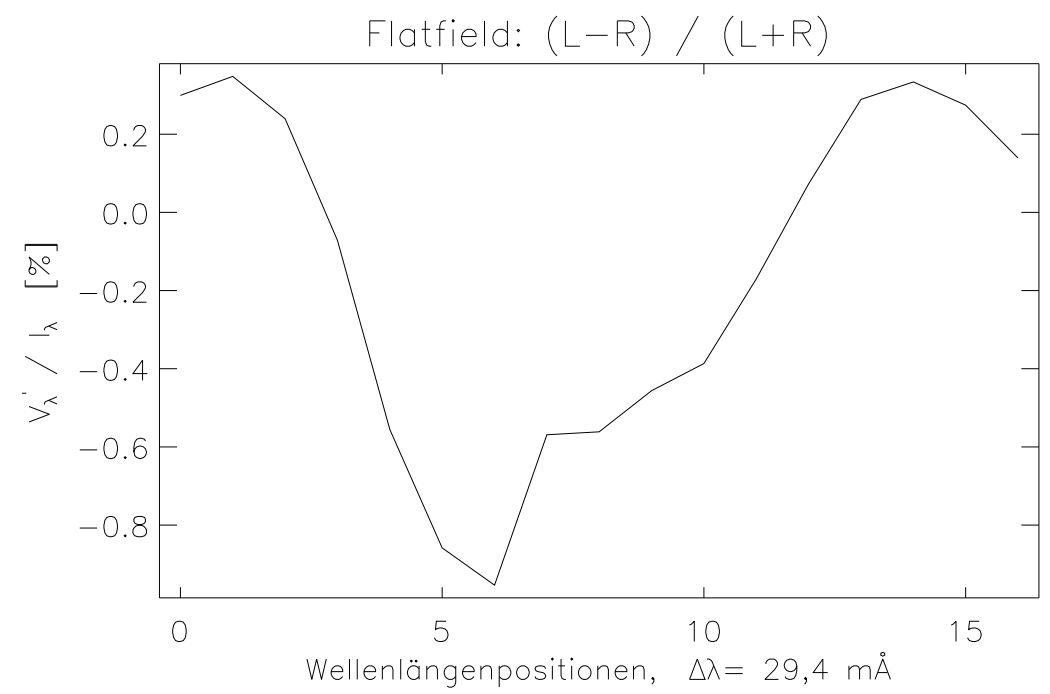

Abbildung 4.2: Wellenlängenabhängige Schwankungen von $V_{\lambda}^{\prime} / I_{\lambda}$ aus den Flatfields. 


\section{Datenreduktion}

Es zeigt sich, dass der aus den Flatfields gewonnene Quotient $V_{\lambda}^{\prime} / I_{\lambda}$ eine Wellenlängenabhängigkeit besitzt, woraus sich schliessen lässt, dass es sich hier nicht nur um instrumentelle Polarisation handelt. Wir vermuten, dass eine geringfügig unterschiedliche Wellenlängenabhängigkeit der beiden Strahlengänge diesen Effekt verursachen könnte. Durch eine Flatfieldkorrektur zweiter Ordnung kann der Effekt behoben werden.

\subsubsection{Flatfieldkorrektur zweiter Ordnung}

Wie in Kap.4.4.2 beschrieben, wird nach der Flatfieldkorrektur ein unverbreitertes Flatfieldprofil erstellt, mit dem die rekonstruierten Daten multipliziert werden. Dieses Flatfieldprofil wird für den rechten und linken Kanal getrennt berechnet. Ein Vergleich der beiden Profile demonstriert, dass sie recht ähnlich, nicht jedoch identisch sind. Die Linieneinsenkung der normierten Profile unterscheidet sich um etwa $1-1,5 \%$ der Kontinuumsintensität, in den unnormierten Profilen ist zu erkennen, dass das weniger tiefe Profil auch ein etwa $1 \%$ höheres Kontinuum aufweist.

Dass es sich bei dieser Erscheinung nicht um Crosstalk von $I$ nach $V$ handeln kann, wurde in Kap. 4.4.1 gezeigt. Die Intensitätsunterschiede in den Flatfieldprofilen kommen daher entweder aus der Optik, dann müssten sie korrigiert werden, weil sie in den Daten ebenfalls enthalten wären, oder sie werden durch nicht exakt gleichmäßige Ausleuchtung oder sogar durch Strukturen auf der Sonne erzeugt. In diesem Fall ist es möglich, dass auch in den Flatfields ein Stokes$V$-Signal zu sehen ist.

Um ein fälschliches, weil in den Flatfields und somit auch in den Daten enthaltenes, Stokes- $V$ Signal aus den Daten herauszukorrigieren, wird die Flatfieldkorrektur zweiter Ordnung verwendet, vgl. Sánchez Almeida \& Martínez Pillet (1994) und Socas-Navarro (1999).

Bei der Korrektur wird davon ausgegangen, dass die Flatfields kleine Fehler der Größe $\delta \ll 1$ besitzen, so dass die Flatfields, mit denen gerechnet wird, in Wahrheit nicht $F_{l}$ und $F_{r}(l$ : linker Kanal, $r$ : rechter Kanal) sind, sondern $F_{l}\left(1+\delta_{l}\right)$ und $F_{r}\left(1+\delta_{r}\right)$. Anstelle der so definierten Stokes-Parameter $I^{S}$ und $V^{s}$,

$$
I^{s}=\frac{L}{F_{l}}+\frac{R}{F_{r}} \text { und } V^{s}=\frac{L}{F_{l}}-\frac{R}{F_{r}}
$$

mit $L=\frac{1}{2}(I+V)$ und $R=\frac{1}{2}(I-V)$ werden durch die erste Flatfielding Prozedur die Werte $I^{1}$ und $V^{1}$ berechnet:

$$
I^{1}=\frac{L}{F_{l}\left(1+\delta_{l}\right)}+\frac{R}{F_{r}\left(1+\delta_{r}\right)} \text { und } V^{1}=\frac{L}{F_{l}\left(1+\delta_{l}\right)}-\frac{R}{F_{r}\left(1+\delta_{r}\right)} .
$$

Nach einer Reihenentwicklung für $\frac{1}{1+\delta}$ und Vernachlässigung der quadratischen und höheren Glieder, dem Einsetzen in die Definition von $I^{S}$, Addition und Subtraktion der zwei Gleichungen und Vernachlässigung des Summanden $-\frac{V^{s}}{I^{s}}\left(\delta_{l}-\delta_{r}\right)$, weil $V^{s} \ll I^{s}$, ergibt sich hieraus

$$
\frac{\left(I^{1}-I^{s}\right)}{I^{s}} \approx-\frac{1}{2}\left(\delta_{l}+\delta_{r}\right) \text { und } \frac{\left(V^{1}-V^{s}\right)}{V^{s}} \approx-\frac{1}{2}\left(\delta_{l}-\delta_{r}\right) \frac{I^{s}}{V^{s}}
$$


Wird diese Formel 4.23 auf Flatfields angewandt, für die das wahre Stokes- $V$-Signal Null sein sollte, entsprechend $V_{f f}^{s} \cong 0$, so folgt

$$
V_{f f}^{1} \approx-\frac{1}{2}\left(\delta_{l}-\delta_{r}\right) I_{f f}^{s},
$$

und man ist in der Lage, den Fehler $\left(\delta_{l}-\delta_{r}\right)$ mit Hilfe der Flatfieldaufnahmen abzuschätzen. Da der relative Fehler des $I^{1}$ nach Formel 4.23 in der Größenordnung von $\delta$ liegt und um den Faktor $\frac{I^{s}}{V^{s}}$ geringer ist als der des $V^{1}$, ersetzt man für die Berechnung des Fehlers das $I_{f f}^{S} \operatorname{durch} I_{f f}^{1}$, so dass sich Formel 4.24 schreiben lässt als

$$
\left(\delta_{l}-\delta_{r}\right) \approx-2 \frac{V_{f f}^{1}}{I_{f f}^{1}} .
$$

Diese Erkenntnis kann nun genutzt werden, um die Daten von dem fälschlich - in den Flatfields und somit auch in den Daten - enthaltenen Stokes- $V$-Profil zu befreien. Durch Einsetzten von Formel 4.25 in Formel 4.23 ergibt sich so das mit einer Flatfield-Korrektur 2. Ordnung versehene Stokes- $V$-Profil der Daten (mit $I^{S} \cong I^{1}$ ) zu

$$
V^{s} \approx V^{1}-\frac{V_{f f}^{1}}{I_{f f}^{1}} I^{1} .
$$

Anschaulich beinhaltet Formel 4.26 nichts anderes, als dass von dem für die Daten bestimmten Stokes- $V$-Signal jenes Stokes- $V$-Signal abgezogen wird, welches bereits in den Flatfields enthalten ist; wobei das Signal mit dem Verhältnis der Kontinuumsintensitäten der Daten zu den Flatfields gewichtet werden muss.

\subsubsection{Magnetfeld aus der Stokes- $V$-Amplitude}

Für jeden Pixel des zweidimensionalen Bildfeldes liegen zwei komplette Wellenlängenprofile von $\frac{1}{2}(I+V)$ und $\frac{1}{2}(I-V)$ vor, so dass für jeden Pixel durch Subtraktion der beiden Kanäle ein komplettes Stokes- $V$-Signal berechnet werden kann. Auf diese Weise werden nun nach der Flatfield-Korrektur 2. Ordnung, vgl. Kap. 4.4.2, Stokes- $V$ - und auch Stokes- $I$-Profile jedes Bildpunktes erstellt.

Bei zunehmender Stärke des Magnetfeldes wächst zunächst die Amplitude des Stokes- $V$-Signales, bis eine Sättigung eintritt, siehe Abb. 4.3. Eine weitere Verstärkung des Magnetfeldes bewirkt ein Auseinanderlaufen der beiden Stokes- $V$-Extrema in Wellenlängenrichtung (Landi Degl'Innocenti 1992). Zur Unterscheidung dieser Fälle kann die sogenannte Weak-Field-Approximation, vgl. Kap. 2.2, genutzt werden.

Betrachtet man vorrangig schwache Felder, die der Weak-Field-Approximation genügen, besteht die Möglichkeit, durch die Höhe der Amplitude des Stokes- $V$-Signales eine Abschätzung des Magnetfeldes, bzw. der Sichtlinienkomponente des Magnetfeldes, zu erhalten. 


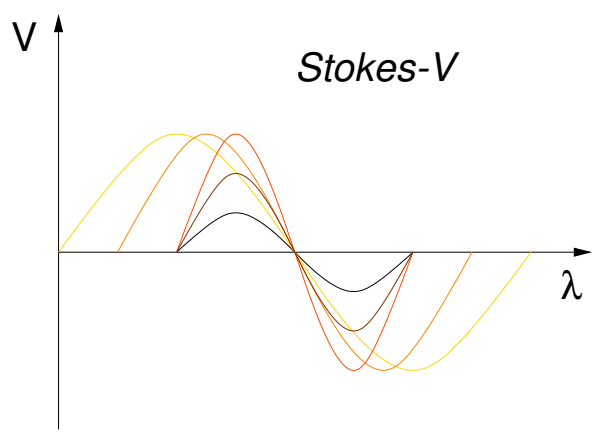

Abbildung 4.3: Skizze der Änderung des Stokes- $V$-Signales mit zunehmender Magnetfeldstärke. Eine hellere Farbe entspricht einem stärkeren Magnetfeld.

In der Praxis werden für die Bestimmung der Stokes- $V$-Amplitude das Maximum und Minimum des Stokes- $V$-Profiles verwendet. Es wird die Hälfte ihrer Differenz, der mittleren Amplitude des Signales, in Prozent der Kontinuumsintensität $I_{c}$ des Ortspunktes ermittelt. In den vorliegenden Daten wurden auf diese Weise Magnetfelder mit Stokes- $V$-Amplituden von $1 \%$ bis zu $12 \% I_{c}$ detektiert.

\subsubsection{Magnetfeld aus Linienschwerpunkten}

Eine zweite, genauere Methode zur Bestimmung des Magnetfeldes, s. Cauzzi et al. (1993), bedient sich nicht des Stokes- $V$-Signales, sondern direkt der Unterschiede, die das Stokes- $V$-Signal in den beiden Kanälen $\frac{1}{2}(I+V)$ und $\frac{1}{2}(I-V)$ hinterlässt. Sie lässt sich auch auf stärkere Magnetfelder, die nicht der Weak-Field-Approximation genügen, anwenden.

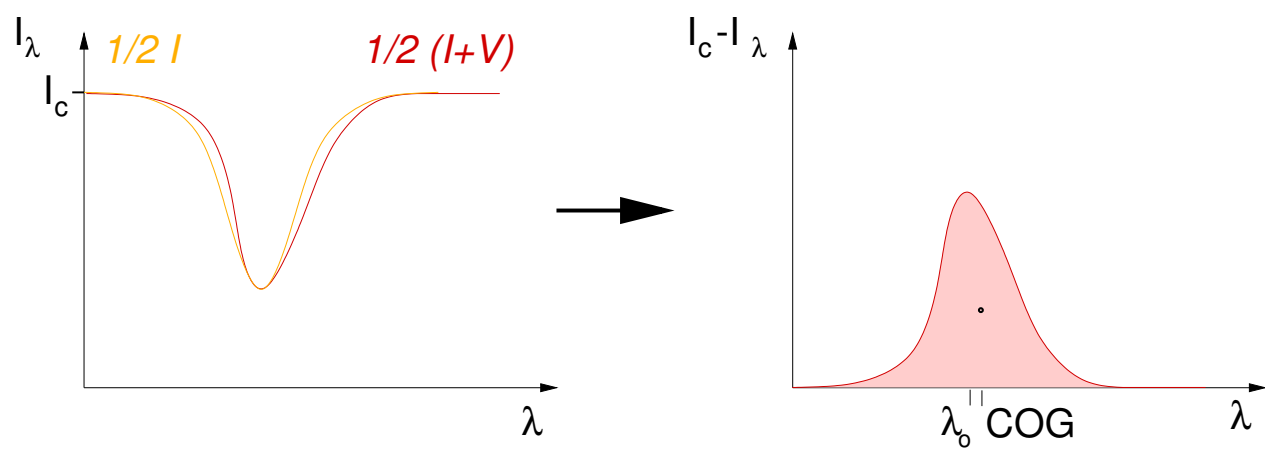

Abbildung 4.4: Skizze zur Bestimmung des Linienschwerpunktes (COG) des $\frac{1}{2}(I+V)$ Signales.

Für die Linienschwerpunktsmethode (engl. Center of Gravity, COG) nach Semel (1967) wird der Schwerpunkt der Absorptionsprofile des $\frac{1}{2}(I+V)$ Kanales und des $\frac{1}{2}(I-V)$ Kanales be- 
stimmt, siehe Abb. 4.4. Aus der Aufspaltung der Schwerpunkte kann über den Zeeman-Effekt, s. Kap. 2.1.1, die Stärke des Magnetfeldes in Tesla bestimmt werden.

Die Berechnung eines Schwerpunktes beruht auf der Verschiebung des Profiles um die Kontinuumsintensität und der anschliessenden Invertierung der Intensitäten.

Eine gute Abschätzung der Kontinuumsintensität ist hierbei wichtig, da nach Verschieben und Umklappen des Profiles alle Werte unter Null ignoriert werden müssen. Eine zu gering geschätzte Kontinuumsintensität würde folglich zur Nichtbeachtung einiger Datenpunkte führen, während eine zu hoch geschätzte Kontinuumsintensität zu einem zusätzlichen Anteil gleichverteilter Werte und somit zu einer Verschiebung des berechneten Schwerpunkts in Richtung der Mitte des Bereiches führen würde. Die Kontinuumsintensität wird daher aus einigen Punkten des Linienkontinuums gemittelt.

Es sei angemerkt, dass mit beiden Methoden, COG und Stokes- $V$-Amplituden, die über das Auflösungselement gemittelte Sichtlinienkomponente des Magnetfeldes gemessen wird (Semel 1967; Landi Degl'Innocenti 1992).

\subsubsection{Korrektur der Intensitätsabhängigkeit}

Um eine Abschätzung für die Zuverlässigkeit und Genauigkeit der COG-Methode zu erhalten, wird die Sichtlinienkomponente des Magnetfeldes gegen die Kontinuumsintensität aufgetragen.
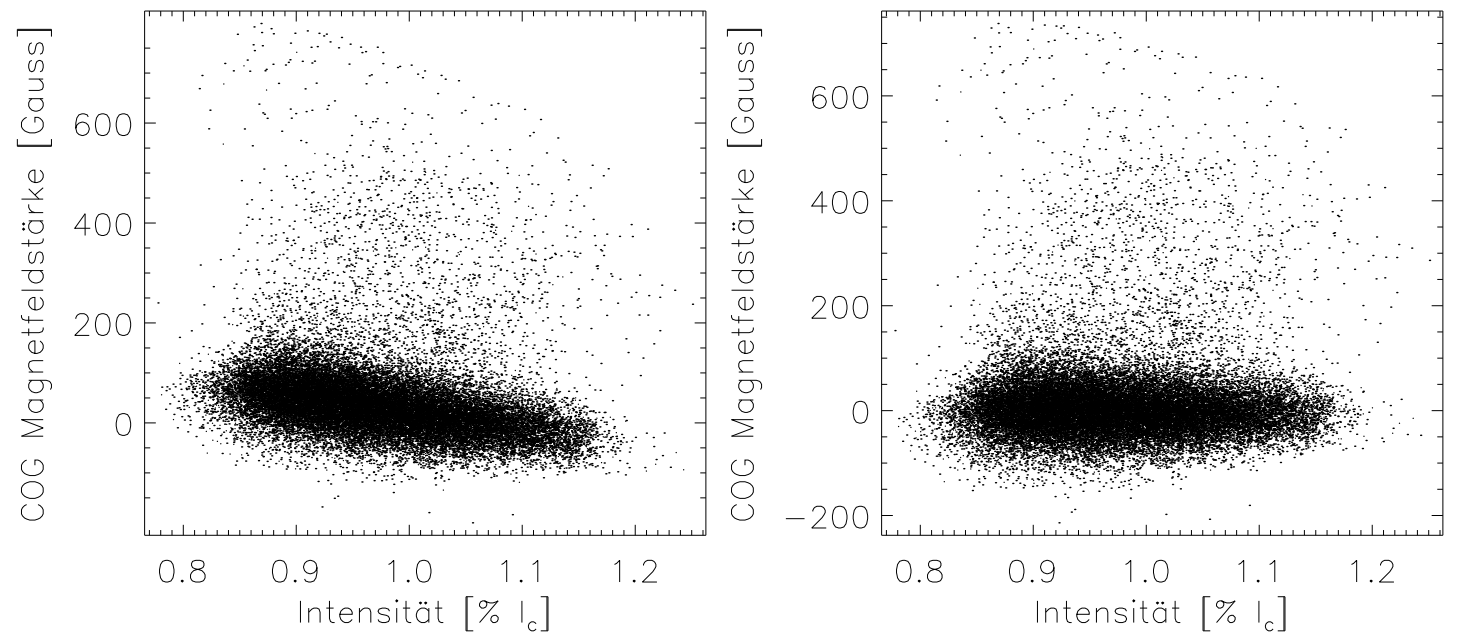

Abbildung 4.5: links: Das Magnetfeld in Abhängigkeit von der Intensität. rechts: Nach der Korrektur des Magnetfeld-Intensitäts-Gradienten.

Zum einen ergibt sich aus dieser Auftragung die Streuung der Magnetfeldstärke zu $\sigma=40$ Gauss, zum anderen muss festgestellt werden, dass in den Daten eine Abhängigkeit des Magnetfeldes von der Intensität vorliegt, vgl. Abb. 4.5 links. Eine Untersuchung mehrerer Serien ergab, dass 


\section{Datenreduktion}

dieser Effekt nicht mit der vorherrschenden Polarität korreliert ist, somit wird ein physikalische Ursache dieses Trends für unwahrscheinlich gehalten. Möglicherweise spielen geringfügige Unterschiede der Schwerpunktsbestimmung bei verschiedenen Intensitäten und in den beiden Kanälen eine Rolle.

$\mathrm{Da}$ in der Auswertung mehrfach Schwellwerte absoluter Magnetfeldstärken benutzt werden, s. Kap. 5.1 und 5.2, und der Realitätsgehalt dieses Gradienten sehr fraglich ist, soll er trotz ungeklärter Ursache korrigiert werden. Für die Korrektur werden alle Bildpunkte mit Magnetfeldstärken von weniger als 200 Gauss herangezogen, um durch lineare Regression die Steigung des Trends zu ermitteln. Der so für alle Intensitäten gegebene Magnetfeld-Offset wird von den berechneten COG-Magnetfeldstärken subtrahiert. Abb. 4.5 rechts zeigt den Erfolg der Korrektur.

\subsection{Geschwindigkeit}

Bei der Berechnung der Magnetfelder durch Stokes- $V$-Amplituden, s. Kap. 4.4.3, oder die Linienschwerpunktsmethode, s. Kap. 4.4.4, bietet sich nahezu als Nebenprodukt auch je eine Methode der Geschwindigkeitsberechnung an. Zum einen kann die Geschwindigkeit aus dem Linenschwerpunkt des Stokes- $I$-Signales, zum anderen aus dem Nulldurchgang des Stokes- $V$-Signales berechnet werden.

In beiden Fällen ergibt sich die Geschwindigkeit aus der Dopplerverschiebung der Wellenlängen, wobei wegen der fehlenden Wellenlängenreferenz nur relative Geschwindigkeiten angegeben werden können.

\subsubsection{Geschwindigkeit aus dem Stokes-I-Schwerpunkt}

Bei der Magnetfeldberechnung nach der COG-Methode werden die Linienschwerpunkte des $\frac{1}{2}(I+V)$ und $\frac{1}{2}(I-V)$ Kanales berechnet, aus denen sich durch einfache Mittelung der Schwerpunkt des Stokes-I-Signales ergibt. Die Verschiebung der Wellenlänge des Stokes- $I$-Schwerpunktes lässt sich nun mit dem Doppler-Effekt nach

$$
v=\frac{\Delta \lambda}{\lambda} \cdot c
$$

direkt in eine Geschwindigkeit umrechnen, sobald eine Referenzwellenlänge gefunden ist, auf die sich die Verschiebung beziehen kann. Da in den vorliegenden Daten keine absoluten Wellenlängen bestimmt werden können, wird als Referenz für die Geschwindigkeitsberechnung der Mittelwert aller Stokes- $I$-Schwerpunkte benutzt.

Das Resultat der Geschwindigkeitsbestimmung aus dem Stokes-I-Schwerpunkt liefert demnach die mittlere der in einem Pixel von $72,5 \times 72,5 \mathrm{~km}^{2}$ enthaltenden Geschwindigkeiten relativ zur mittleren Geschwindigkeit des gesamten Bildfeldes. 


\subsubsection{Geschwindigkeit aus dem Stokes- $V$-Nulldurchgang}

Das für die Magnetfeldberechnung aus der Stokes- $V$-Amplitude erstellte Stokes- $V$-Profil kann ebenfalls zur Gewinnung einer relativen Geschwindigkeit herangezogen werden.

Grundlage dieser Methode ist es, die Wellenlängenverschiebung des Nulldurchganges (engl. zerocrossing) des Stokes- $V$-Profiles zu ermitteln. Hierzu wird der Bereich zwischen dem Minimum und dem Maximum des Stokes- $V$-Signales durch ein Polynom dritten Grades gefittet und durch Iteration der Regula Falsi dessen Nulldurchgang bestimmt.

Die nach Formel 4.27 aus der Dopplerverschiebung des Nulldurchganges gewonnene Geschwindigkeit entspricht wie auch die aus dem Linienschwerpunkt des Stokes-I-Signales berechnete Geschwindigkeit der Geschwindigkeit der Strukturen in der mittleren Entstehungshöhe der Linie. Da jedoch das Stokes- $V$-Signal ausschliesslich in magnetischen Strukturen gebildet wird (der Stokes-I-Anteil der beiden Kanäle wird für die Bestimmung von Stokes- $V$ ja gerade herausgerechnet), entspringen die hieraus gebildeten Geschwindigkeiten einzig den magnetischen Strukturen.

Die Geschwindigkeiten aus dem Nulldurchgang des Stokes- $V$-Profiles beschreiben folglich die mittlere Geschwindigkeit der in einem Pixel von $72,5 \times 72,5 \mathrm{~km}^{2}$ enthaltenen magnetischen Strukturen relativ zur mittleren Stokes-I-Geschwindigkeit aller Strukturen des Bildfeldes. 
4 Datenreduktion 


\section{Ergebnisse}

\subsection{Die Hausdorff-Dimension magnetischer Strukturen}

Die Hausdorff-Dimension einer Struktur ist eine gebrochene Dimensionsangabe, die eine Aussage über die Form und Beschaffenheit dieser Struktur macht. Für gebrochene Dimensionen wie die Hausdorff-Dimension wird auch der Begriff der fraktalen Dimension benutzt.

Um sich eine Hausdorff-Dimension anschaulich vorstellen zu können, betrachte man zuerst einen Strich auf dem Papier. Dieser hat die Dimension eins, während jede Fläche die Dimension zwei besitzt. Aber könnte man einem unendlich oft abgeknickten Strich, der einen großen Teil der Fläche bedeckt, nicht auch eine Dimension zwischen eins und zwei, eine fraktale Dimension, zuordnen?

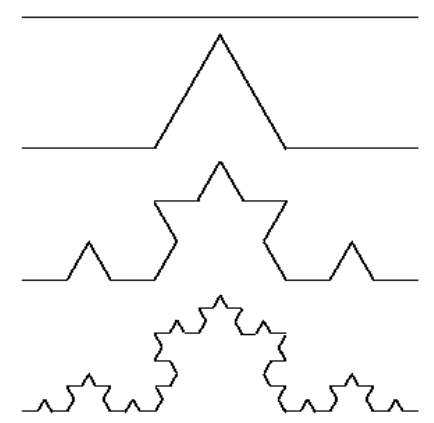

Abbildung 5.1: Die 0. bis 3. Iteration der triadischen Kochkurve als Beispiel einer Struktur, deren Grenzobjekt die Dimension $D=\frac{\log 4}{\log 3} \approx 1,2619$ besitzt.

Als Beispiel diene die triadische Kochkurve. Sie wird gebildet, indem das mittlere Drittel einer Geraden herausgeschnitten und durch eine Spitze aus zwei Drittelstücken ersetzt wird. In unendlich vielen Iterationen wird dieses für jede der Teilgeraden wiederholt, siehe Abb. 5.1. Jede einzelne dieser Iterationen besitzt die Dimension eins. Dem Grenzobjekt, der unendlich oft iterierten Kochkurve, kann jedoch eine fraktale Dimension zugewiesen werden, sie beträgt $D=\frac{\log 4}{\log 3}$. 


\section{Ergebnisse}

\subsubsection{Motivation}

Die auffällige Form und Verteilung magnetischer Strukturen der Sonne ist Thema einiger neuer Veröffentlichungen von Stenflo \& Holzreuter (2003a b c). Sie vergleichen Magnetogramme unterschiedlicher Größenskalen - Kitt Peak Magnetogramme, MDI Magnetogramme von SOHO und hochaufgelöste Magnetogramme des Schwedischen Sonnenteleskopes (SVST) auf La Palma - und stellen fest, dass sich die Formen der Strukturen aller Magnetogramme auf frappierende Weise ähneln: Alle magnetischen Strukturen zeigen dieselbe zerrupfte Form. Wechselt man zu einem Magnetogramm mit besserer Auflösung, so zeigt sich wieder das gleiche Bild ausgefranster Strukturen. Stenflo \& Holzreuter (2003b) zeigen, dass die WahrscheinlichkeitsVerteilungsfunktionen der magnetischen Feldstärken für alle Auflösungen erstaunlich gut übereinstimmen. Erklärt wird dies durch die Turbulenz der Magnetokonvektion. Die Autoren vermuten, dass die Selbstähnlichkeit der Strukturen bis zur Dissipation aufrecht erhalten bleibt.

Über die Selbstähnlichkeit granularer Strukturen gibt es bereits einige Veröffentlichungen. So haben Roudier \& Muller (1987) die Dimension der Ränder von kleineren Granulen zu $D=1,25$ und die der größeren zu $D=2,15$ bestimmt. Sie geben eine kritische Größe der Granulendurchmesser von $d=1,37^{\prime \prime}$ an, bei der die Dimension umspringt. Eine ähnliche Untersuchung wurde von Greimel et al. (1990) durchgeführt, sie finden ebenfalls $D=1,3$ für kleinere und $D=2,15$ für größere Granulen. Hirzberger et al. (1997) erhalten eine Abhängigkeit der Dimension von der Größe der Granulen mit Dimensionen zwischen $D=1,1$ und $D=2,1$. Sie unterteilen die Granulation in kleinere Strukturen von $d \leq 1,4^{\prime \prime}$ mit $D=1,3$ und größere Strukturen von $d \geq 1,7^{\prime \prime}$ mit $D \approx 2$ und weisen darauf hin, dass eine Dimension von $D=\frac{4}{3}$ für isotrope, homogene Turbulenzen in einer isobaren Atmosphäre erwartet würde. Alle genannten Autoren finden einen Bruch der Selbstähnlichkeit für Granulen in der Größenordnung von $d=1,3^{\prime \prime}-1,8^{\prime \prime}$. Bovelet $\&$ Wiehr (2001) dagegen benutzen eine andere Methode zur Detektion der Einzelstrukturen. Sie verwenden einen Multi-Level-Algorithmus, der das Zusammenkleben nicht zusammengehöriger Strukturen unterbinden soll und erhalten hiermit eine sehr geringe Dimension von $D=1,09$ für alle Größenordnungen der Granulation.

Auch Hirzberger et al. (1997) erhalten eine geringere Dimension von $D=1,16$ ohne Abhängigkeit von der Größe der Strukturen. Dies jedoch nur, wenn sie als Kanten der Granulen die Intensitätsminima des Intergranulums benutzen. Das Ergebnis zeigt, dass die intergranularen Gebiete recht glatt sind.

Über die fraktale Dimension magnetischer Strukturen existieren ebenfalls einige Untersuchungen, wie etwa von Tarbell et al. (1990); Schrijver et al. (1992); Balke et al. (1993). Diese Arbeiten unterscheiden sich jedoch dadurch von der hier vorgestellten, dass die Autoren eine andere Definition der fraktalen Dimension benutzten. Anstelle der hier verwendeten Flächen-UmfangsRelation zur Bestimmung der fraktalen Dimension $D$ des Randes von Strukturen, vgl. Kap. 5.1.3, Formel 5.6, benutzen sie die Definition

$$
L^{D^{\prime}} \propto A
$$

oder entsprechend

$$
D^{\prime} \cdot \log L=\log A+\text { const }^{\prime},
$$


die die fraktale Dimension $D^{\prime}$ der Fläche der Struktur wiedergibt. Diese Definition wird angewandt, indem für einen festen Schwellwert des Magnetfeldes die Pixel der Fläche $A$ gezählt und logarithmisch gegen die Länge $L$ des kleinstmöglichen Quadrates aufgetragen werden, das die Struktur komplett beinhaltet. Der Unterschied wird für einfache Strukturen wie Kreise oder Quadrate sofort deutlich. Die Dimension der Fläche bestimmt sich in diesem Fall zu einem Wert von $D^{\prime}=2$, während die in dieser Arbeit benutzte Definition der Dimension des Randes den Wert $D=1$ liefert.

Mit dieser Flächen-Definition finden zunächst Tarbell et al. (1990) eine fraktale Dimension von $D^{\prime}=1,6$ für Strukturen aller Größenordnungen, d.h. $0,16^{\prime \prime}<L<10^{\prime \prime}$, während Schrijver et al. (1992) den Größenbereich einschränken auf $0,2^{\prime \prime}<L<5^{\prime \prime}$ und für diese Strukturen einen Dimensionsbereich von 1,45<D $<1,60$ angeben. Balke et al. (1993) bestimmen für kleine magnetische Strukturen mit $L \leq 3^{\prime \prime}$ eine Dimension der Fläche von $D^{\prime}=1,54$, wobei sie zudem erklären, dass für die Strukturen unterschiedlicher Größenordnungen keine einheitliche Dimension angegeben werden könnte. Zu diesem Ergebnis kommt auch Meunier (1999), die in ihrer Arbeit neben der Dimension der Fläche auch die des Randes bestimmt. Bei einem Schwellwert von 40 Gauss findet sie abhängig von der Größe der Struktur Flächendimensionen von $D^{\prime}=1,78$ bis 1,94 und Dimensionen des Randes von $D=1,48$ bis 1,68 .

Im Folgenden soll nun für hochaufgelöste Magnetfeldkarten der Rand magnetischer Strukturen untersucht und für ihn die Hausdorff-Dimension berechnet werden.

\subsubsection{Mathematische Definition}

Um den Begriff der Hausdorff-Dimension mathematisch erfassen zu können, wird für jeden Parameter $s>0$ ein sogenanntes äußeres Maß $H^{s}$ eingeführt (Falconer[1990, S. 25ff). Die Definition dieses äußeren Maßes der Teilmenge $E \subset \mathbb{R}^{n}$ wiederum basiert auf dem Wert

$$
H_{\varepsilon}^{s}(E):=\inf _{\left(U_{i}\right)_{i \in \mathbb{N}}}\left(\sum_{i=1}^{\infty}\left|U_{i}\right|^{s}\right),
$$

der für jedes $\varepsilon>0$ das obige Infimum über alle möglichen $\varepsilon$-Überdeckungen $\left(U_{i}\right)_{i \in \mathbb{N}}$ der Menge $E$ darstellt. Hierbei ist eine $\varepsilon$-Überdeckung $\left(U_{i}\right)_{i \in \mathbb{N}}$ eine Folge von Teilmengen $U_{i} \subset \mathbb{R}^{n}$ mit $E \subset \bigcup_{i=1}^{\infty} U_{i}$, wobei die Durchmesser $\left|U_{i}\right|$ der Teilmengen

$$
\left|U_{i}\right|:=\operatorname{diam}\left(U_{i}\right)=\sup \left\{|x-y|, x, y \in U_{i}\right\}
$$

nicht größer als $\varepsilon$ sein dürfen, $\left|U_{i}\right| \leq \varepsilon$.

Das sogenannte $s$-Hausdorffmaß $H^{s}(E)$ ist dann definiert als das Supremum von $H_{\varepsilon}^{s}(E)$ für $\varepsilon$ gegen Null:

$$
H^{s}(E):=\sup _{\varepsilon>0} H_{\varepsilon}^{s}(E)=\lim _{\varepsilon \rightarrow 0} \inf _{\left(U_{i}\right)}\left(\sum_{i=1}^{\infty}\left|U_{i}\right|^{s}\right)
$$




\section{Ergebnisse}

Für die Berechnung des $s$-Hausdorffmaßes $H_{\varepsilon}^{s}(E)$ werden also Überdeckungen mit immer kleinerem Durchmesser der überdeckenden Mengen betrachtet. Es läßt sich zeigen, dass die Abbildung $H^{s}: \mathbb{R}^{n} \rightarrow \mathbb{R}$ für jedes $\varepsilon>0$ ein äußeres Maß auf $\mathbb{R}^{n}$ definiert (Falconer 1990). Der Wert von $H^{s}$ hängt für eine feste Menge $E$ entscheidend von $s$ ab und berechnet sich für fast alle $s$ zu Null oder zu Unendlich.

Für jedes $E$ existiert aber genau ein Wert $\operatorname{dim}_{H}(E)$, für den gilt

$$
\begin{aligned}
& H^{s}(E)=\infty \quad \text { falls } \quad 0 \leq s<\operatorname{dim}_{H}(E) \\
& H^{s}(E)=0 \text { falls } \operatorname{dim}_{H}(E)<s<\infty .
\end{aligned}
$$

Dieses $\operatorname{dim}_{H}(E)$ wird Hausdorff-Dimension der Menge $E$ genannt. Die Hausdorff-Dimension ist damit der Wert $s$, für den der Wert des Hausdorffmaßes $H^{s}(E)$ von Unendlich auf Null springt.

Die Hausdorff-Dimension mathematisch "glatter" Mengen entspricht dem "gewohnten” Dimensionsbegriff, so haben z.B. Kurven die Hausdorff-Dimension eins. Dies bedeutet, dass die Hausdorff-Dimension auch für alle realen, physikalischen Gebilde stets ganzzahlige Werte liefert. Mathematisch definierte Mengen, die z.B. durch einen Grenzprozess definiert werden wie in Abb. 5.1 gezeigt, können dagegen auch nichtganzzahlige Hausdorff-Dimensionen besitzen. Durch Idealisierung ist es daher dennoch möglich, auch realen, physikalischen Gebilden eine nichtganzzahlige Hausdorff-Dimension zuzuweisen.

\subsubsection{Reale Strukturen}

Die Berechnung der Hausdorff-Dimension des Randes magnetischer Strukturen der Sonne beruht nun auf der Benutzung eines Trickes, der aus der Verwendung eines Maßstabes der Länge $G$ besteht (Mandelbrot 1997, S. 109), d.h. dass alle Größenangaben in Vielfachen dieser Länge $G$ ausgedrückt werden. Mit der Flächen-Umfangs-Relation ist es dann möglich, für zweidimensionale Gebilde eine Hausdorff-Dimension ihres Randes anzugeben.

Die Flächen-Umfangs-Relation besagt, dass für die Fläche $A$ und ihren Umfang $P$ geometrisch ähnlicher Körper

$$
P^{\frac{1}{D}}=\text { const }_{1} \cdot A^{\frac{1}{2}}
$$

gilt, sofern $A$ und $P$ in Einheiten von $G$ gemessen werden (Mandelbrot 1997). $D$ gibt dann die fraktale Dimension der Flächenränder an. Umgeformt ergibt sich der einfache Zusammenhang von

$$
\log (P)=\frac{D}{2} \cdot \log (A)+\text { const }_{2} .
$$

In der logarithmischen Auftragung der Flächen gegen die Umfänge zweidimensionaler Strukturen ergibt sich die Hausdorff-Dimension der Ränder dieser Strukturen daher, falls diese geometrisch ähnlich sind, aus der doppelten Steigung der Geraden. Die Ränder von Strukturen, die 
nicht geometrisch ähnlich sind, besitzen unterschiedliche Dimensionen, so dass die Punkte der Flächen-Umfangs-Relation nicht auf einer Geraden liegen. In diesem Fall ließe sich keine einheitliche Dimension bestimmen.

Die Methode der Dimensionsbestimmung anhand der Flächen-Umfangs-Relation wurde erstmals von Lovejoy (1982) verwendet, um die Hausdorff-Dimension der Ränder von Wolken zu bestimmen. In der Folgezeit ist sie von vielen Astrophysikern aufgegriffen worden (s. Kap. 5.1.1).

\subsubsection{Praktische Umsetzung}

Für die Erstellung der Flächen-Umfangs-Relation, müssen zunächst aus den zweidimensionalen Magnetfeldkarten Flächen gleichen Magnetfeldes ausgeschnitten werden. Der Schwellwert des Magnetfeldes beeinflusst hierbei die Größe und Anzahl der gefundenen Strukturen erheblich, vgl. Kap. 5.1.10.

Die Detektionsgrenze des Magnetfeldes wird durch Auftragung der gemessenen Sichtlinienkomponente des Magnetfeldes gegen die Intensität der zugrunde liegenden Struktur und Anfittung einer Gausskurve auf $\sigma=40$ Gauss abgeschätzt. Um für die Bestimmung der HausdorffDimension im sicheren Bereich zu liegen, wird - sofern nichts anderes angegeben ist - im Folgenden ein Wert von 80 Gauss als Schwellwert für das Magnetfeld benutzt. Mit Hilfe eines modifizierten Algorithmus von Johann Hirzberger (1998) wird eine Schwarzweiß-Maske der Magnetfelder erstellt, in der die Flächen und Umfangspixel der Strukturen einzeln identifiziert und indiziert sind, vgl. Abb. 5.2 unten.

Auswahlkriterien der Strukturen Nicht alle der Strukturen, die nach obigem Verfahren gefunden werden, können für die Berechnung der Hausdorff-Dimension herangezogen werden. Um mögliche Fehlerquellen auszuschließen, werden folgende Strukturen nicht betrachtet:

- Strukturen, die den Rand des Bildfeldes berühren, werden nicht berücksichtigt. Diese Strukturen werden entfernt, da über ihre Fortsetzung über den Bildrand hinaus keine klare Aussage möglich ist. Es sind Versuche unternommen worden, diese Strukturen als abgeschlossen oder als geöffnet zu betrachten, die kurz in Kap. 5.1.13 vorgestellt werden. Als korrekte Lösung wird jedoch angesehen, diese Strukturen bei der Berechnung der Dimension zu ignorieren. Im Folgenden wird daher ausschließlich mit Strukturen gearbeitet, die den Bildfeldrand nicht berühren.

- Sehr kleine Strukturen werden ebenfalls nicht berücksichtigt. Für eine sinnvolle Berechnung der fraktalen Dimension ist eine gewisse Größe der Strukturen unerlässlich. Dies wird für die kleinsten Strukturen sofort klar: Eine Fläche von einem Pixel besitzt immer einen Umfang von vier Einheiten, Flächen von zwei Pixeln haben sechs, drei Pixel haben acht Einheiten im Umfang. Aus diesen Strukturen ist folglich keine Aussage über die Dimension ihres Randes zu gewinnen. Für vier oder fünf Flächenpixel sind je nach Form der Struktur zwei verschiedene Anzahlen an Umfangseinheiten möglich, auch diese 


\section{Ergebnisse}
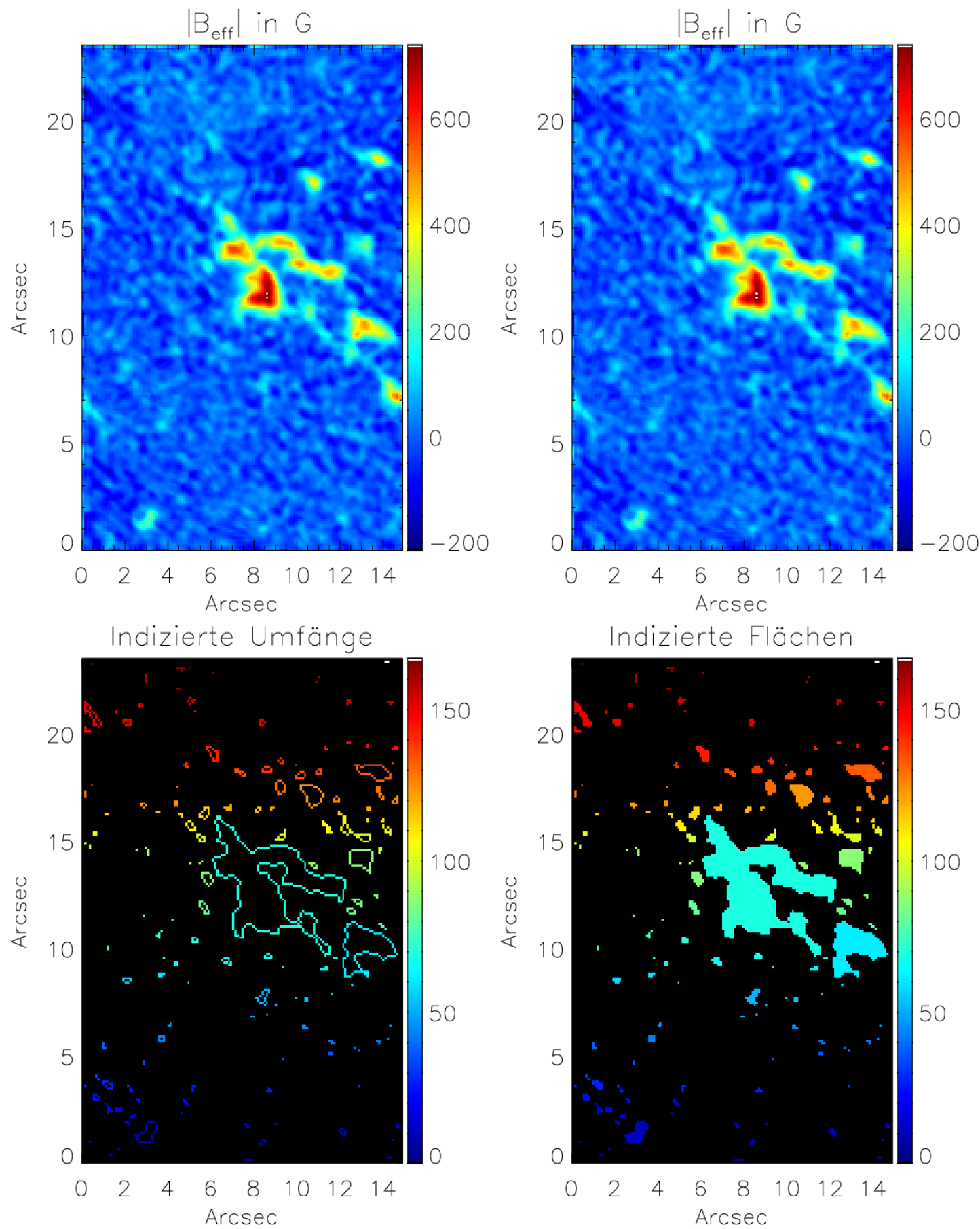

Abbildung 5.2: oben: Sichtlinienkomponente des Magnetfeldes (doppelt), unten: Indizierte Flächen- und Umfangspixel. 
Strukturen werden für die Auswertung nicht verwendet. Es werden testweise auch Strukturen mit weniger als acht Flächenpixeln ausgeschlossen, die drei verschiedene Anzahlen an Umfangseinheiten besitzen können. In diesem Punkt muss jedoch abgewogen werden zwischen der Größe der ausgeschlossenen Strukturen und der Gesamtanzahl auswertbarer Strukturen. Da die mit einer Grenze von acht Flächenpixeln erzielten Ergebnisse qualitativ mit denen aus sechs Flächenpixeln identisch waren, werden sechs Flächenpixel als minimale Größe festgesetzt.

Flächenpixel und Umfangseinheiten der verbleibenden Strukturen werden in Einheiten des $G$ Maßstabes, der Pixelseitenlänge, ausgezählt, und nach Formel 5.6 logarithmisch gegeneinander aufgetragen. Fig. 5.3 zeigt exemplarisch die Berechnung der Hausdorff-Dimension des Randes der magnetischen Strukturen aus Scan 11/6 bei $\cos \theta=0,82$.

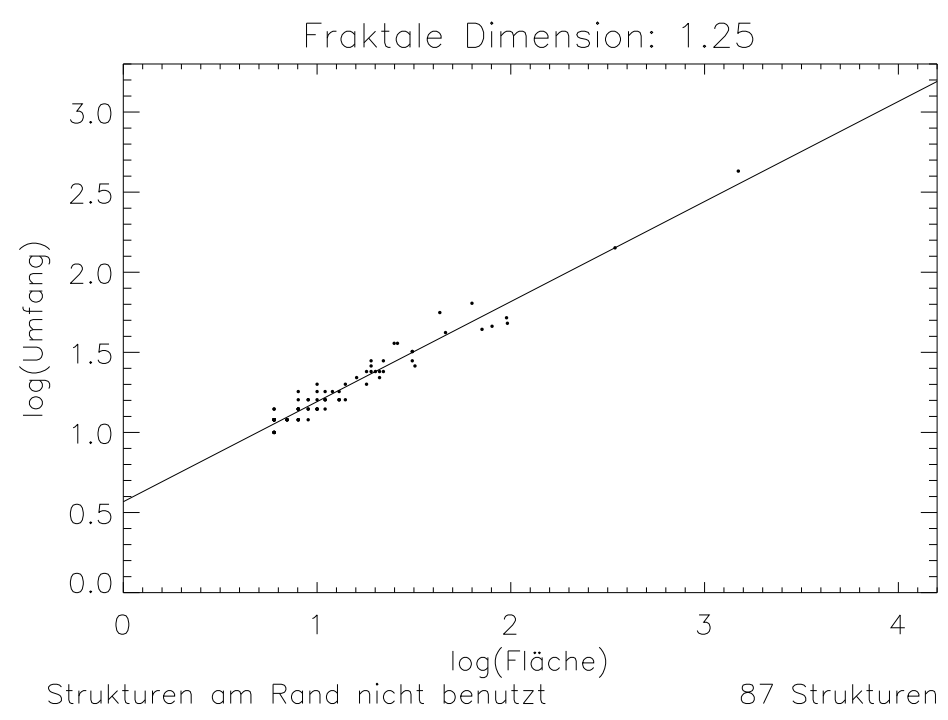

Abbildung 5.3: Bestimmung der Hausdorff-Dimension von Magnetischen Strukturen größer 80 Gauss aus einem einzelnen Scan.

Für den Schwellenwert des Magnetfeldes bei 80 Gauss werden in diesem Scan 223 Strukturen gefunden, von denen 34 Strukturen den Rand des Bildfeldes berühren und 102 Strukturen weniger als sechs Pixel enthalten, so dass sie nicht berücksichtigt werden. Die Flächen und Umfänge der verbleibenden 87 Strukturen werden logarithmisch gegeneinander aufgetragen und für die Berechnung einer Regressionsgeraden benutzt. Die Steigung dieser Geraden beträgt 0,625, so dass sich für den Rand der magnetischen Strukturen dieses Beispiel-Scans eine Hausdorff-Dimension von 1,25 ergibt.

\subsubsection{Mitte-Rand-Variation}

Im August 2000 wurden zur Untersuchung der Mitte-Rand-Variation kleinskaliger magnetischer Strukturen 8 Serien von je 15 Scans an verschiedenen $\cos \theta$-Positionen aufgezeichnet. Bestimmt 


\section{Ergebnisse}

man für jede dieser Serien eine fraktale Dimension, so ergibt sich die in Abb. 5.4 dargestellte

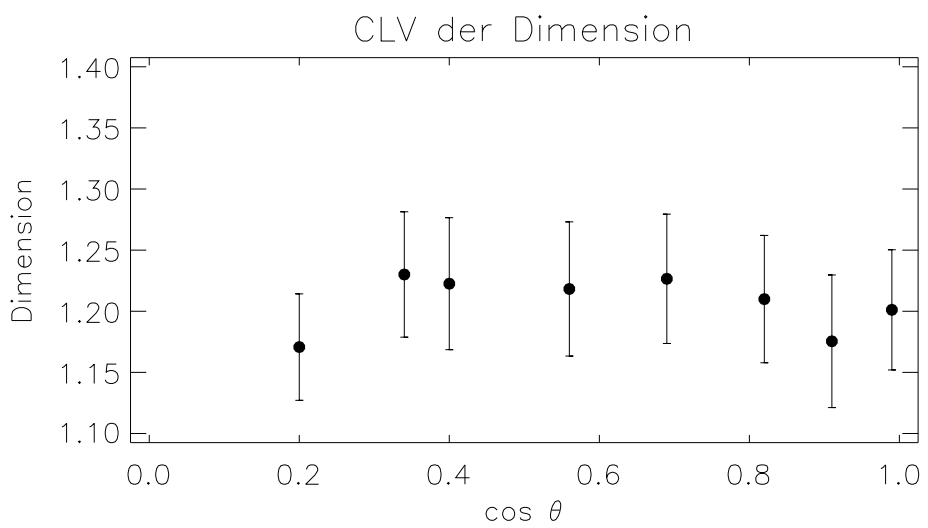

Abbildung 5.4: Mitte-Rand-Variation der Hausdorff-Dimension.

Mitte-Rand-Variation der Dimensionen. Jede dieser Dimensionen wird hierbei aus der Zusammenfassung aller Strukturen einer solaren Position, d.h. aus 15 Scans, in einer Flächen-UmfangsRelation bestimmt.

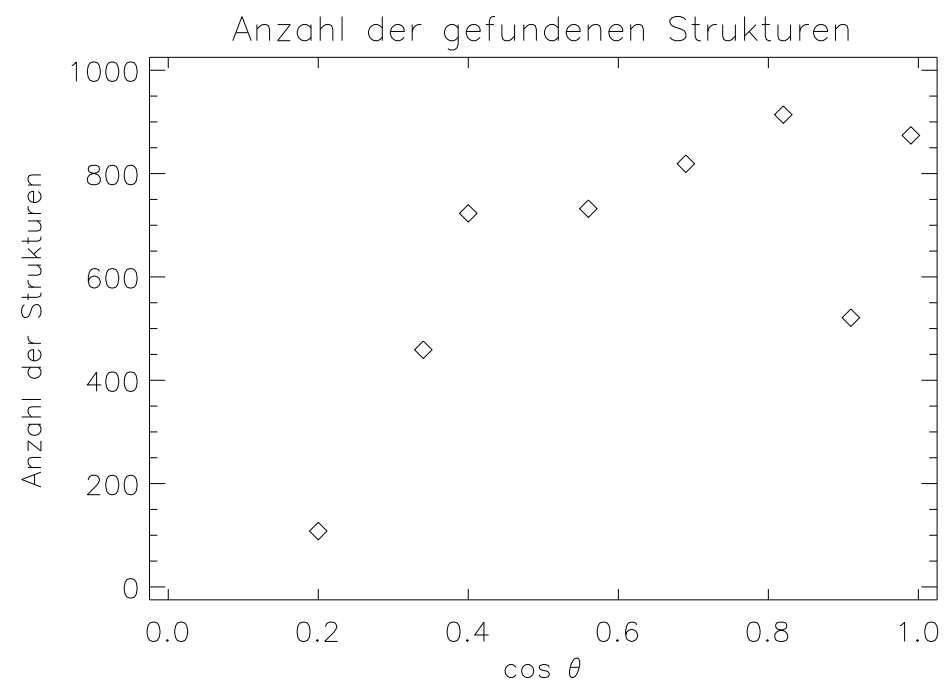

Abbildung 5.5: Anzahl der gefunden Strukturen pro Serie.

Abb. 5.5 zeigt die Anzahl der für die Berechnung zur Verfügung stehenden Strukturen pro solarer Position. Sie variiert von 914 in der Sonnenmitte bei $\cos \theta=0,82$ bis zu 108 am Sonnenrand bei $\cos \theta=0,20$. Alle untersuchten solaren Positionen zeigen Plage oder Fackelgebiete, bis auf die Serie bei $\cos \theta=0,91$. Diese Position enthält zusätzlich zwei kleine Poren. 
In der Abb. 5.4 erkennt man keine Abhängigkeit der Hausdorff-Dimension von der Position auf der Sonnenscheibe. Die Strukturen scheinen ihre Form in den am Sonnenrand untersuchten, etwa $50-100 \mathrm{~km}$ höher gelegenen Schichten noch nicht geändert zu haben. Der niedrigere Wert bei $\cos \theta=0,20$ wird aus einer geringeren Anzahl von Strukturen berechnet und birgt daher einen größeren Unsicherheitsfaktor. Doch selbst inklusive dieses Ergebnisses liegen alle Dimensionen in einem Rahmen von $\pm 0,03$ mit dem geringsten Wert von $D=1,17$ bei $\cos \theta=0,20$ und dem maximalen $D=1,23$ bei $\cos \theta=0,34$. Aufgrund der relativ geringen Schwankung und der nicht vorhandenen Abhängigkeit der Dimension von der Position auf der Sonnenscheibe lässt sich zweifelsfrei die Vereinigung aller Serien und Scans in einer Flächen-Umfangs-Relation legitimieren.

\subsubsection{Beobachtete Magnetfelder}

Da die Mitte-Rand-Variation der Dimension in einem sehr engen Rahmen von $\Delta D= \pm 0,03$ liegt, ist es gerechtfertigt, aus den beobachteten Strukturen aller Positionen eine mittlere Dimension zu ermitteln, um die Signifikanz der Statistik zu erhöhen. Durch die Benutzung aller 120 Scans steht somit die deutlich größere Stichprobe von 5150 Strukturen zur Verfügung. Fig. 5.6zeigt die Flächen-Umfangs-Relation dieser größeren Stichprobe, aus der sich die fraktale Dimension der beobachteten Magnetfelder zu $D_{b}=1,21$ bestimmt.

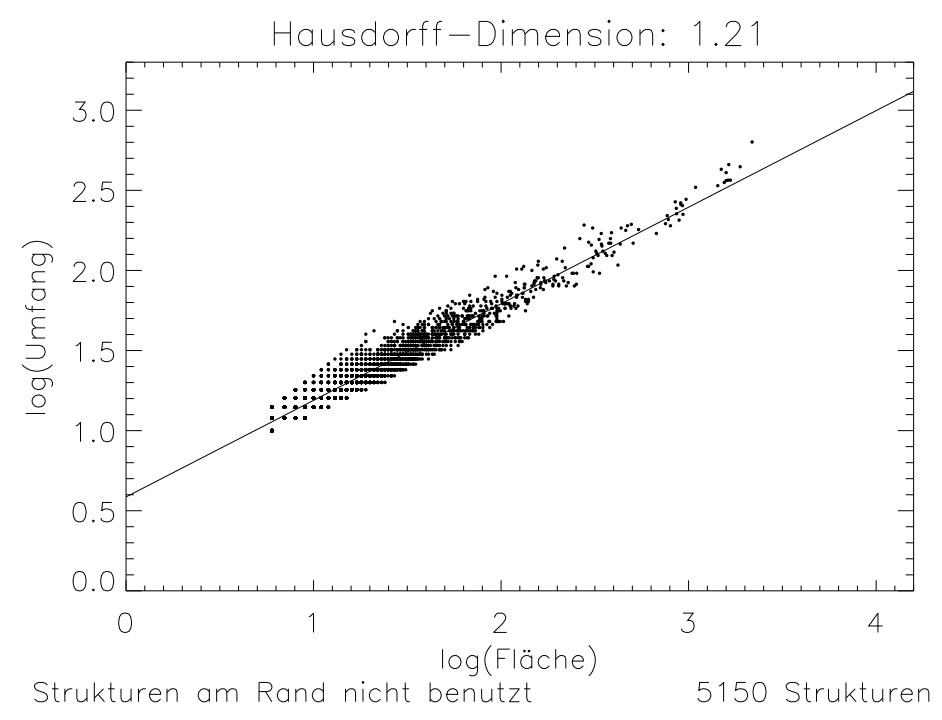

Abbildung 5.6: Fraktale Dimension aus 120 beobachteten Scans. Die Stichprobe von 5.150 Strukturen ergibt eine Dimension von 1,21.

Für sehr große Strukturen liegt ein leichter Trend zu größeren Dimensionen vor, ein eindeutiger Abknickpunkt lässt sich allerdings nicht angeben. Auf die Änderung der Dimension für sehr große Strukturen wird in Kap. 5.1.14 eingegangen. 


\section{Ergebnisse}

\subsubsection{Simulierte Magnetfelder}

Von besonderem Interesse bei dieser Untersuchung ist nun die Möglichkeit, die für beobachtete Daten bestimmte Hausdorff-Dimension mit einer Dimension aus magnetischen Strukturen numerischer Simulationen vergleichen zu können. Hierfür werden von Vögler \& Schüssler (2003) zehn stochastisch unabhängige Momentaufnahmen ihrer MHD-Simulationen zur Verfügung gestellt. Die Aufnahmen zeigen, wie auch die beobachteten Daten, die vertikale Magnetfeldkomponente. Sie werden in einer Höhe von $z=0$ in der Modellatmosphäre, d.h. nahe der optischen Tiefe $\tau_{5000}=1$, berechnet und basieren auf einem mittleren vertikalen Magnetfeld von 50 Gauss. Für näheres zu den MHD-Simulationen, siehe Vögler (2003); Schüssler (2003).

In Bogensekunden gemessen ist das Bildfeld dieser Simulationen im Vergleich zu dem der beobachteten Daten deutlich kleiner, dafür allerdings von wesentlich besserer Auflösung. Es umfasst $288 \times 288$ Pixel, wobei ein Pixel eine Größe von 0,0287", entsprechend 20,83 km, besitzt. Die Gesamtbreite des Bildfeldes beträgt somit knapp 8, $3^{\prime \prime}$ oder $6.000 \mathrm{~km}$.

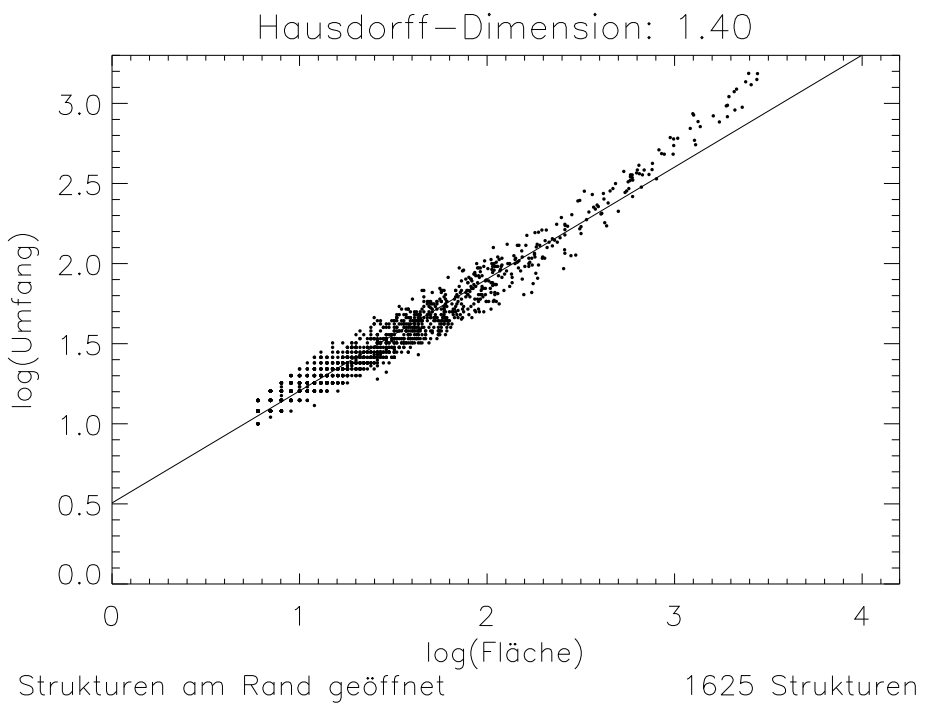

Abbildung 5.7: Bestimmung der Hausdorff-Dimension der Ränder magnetischer Strukturen von mehr als 80 Gauss aus zehn unabhängigen Momentaufnahmen der numerischen Simulation von Alexander Vögler in der Höhe $z=0$.

Da die Vergleichsfelder der numerischen Rechnung an den Rändern periodisch sind, wird der Programmcode zur Bestimmung der Hausdorff-Dimension an diese Rahmenbedingung angepasst. Zwei oder mehr Strukturen, die an entgegengesetzten Bildrändern liegen, werden als eine Struktur erkannt, sofern sie sich über den Rand hinaus berühren.

Die insgesamt 1.625 Strukturen, die auf diese Weise gefunden werden, sind in Bild 5.7 dargestellt. Aus ihnen berechnet sich die Hausdorff-Dimension der Ränder numerisch simulierter Magnetfelder zu $D_{s}=1,40$. 
Die Abweichung der Dimensionen, die aus Simulation und Beobachtung gewonnen werden, ist signifikant. Es wirft sich daher die Frage auf, wie sich der Unterschied erklären lässt.

Das Verhältnis des $G$-Maßstabes zu der Auflösung der betrachteten Daten ist hier von ungeheurer Wichtigkeit. Dieses lässt sich leicht daraus ersehen, dass jede Struktur, die eine Auflösung von einigen Bogensekunden hätte und mit einem Maßstab von $0,1^{\prime \prime}$ gemessen würde, rund erscheinen und somit die Dimension 1,0 besitzen würde. Durch die Benutzung eines $G$-Maßstabes, der kleiner als das Auflösungsvermögen der Daten ist, wird bei der Berechnung der Dimension der beobachteten Daten demzufolge ein Fehler gemacht. Dieser Fehler ist in der aus der numerischen Simulation bestimmten Dimension nicht enthalten, so dass sich möglicherweise hieraus die Diskrepanz der Ergebnisdimensionen ableiten lässt.

\subsection{8 Änderung des G-Maßstabes}

Die These, dass die für die beobachteten Magnetfelder bestimmte fraktale Dimension von $D_{b}=$ $1,21 \mathrm{zu}$ gering gemessen wird, weil der benutzte $G$-Maßstab von einem Pixel, entsprechend $0,105^{\prime \prime}$ Länge, zu fein für die Auflösung der Daten ist, soll untersucht werden. Hierfür wird der benutzte $G$-Maßstab auf vier Pixel hochgesetzt, denn die räumliche Auflösung der Magnetogramme beträgt etwa $0,4^{\prime \prime}$. Die Magnetfeldstärken von jeweils $4 \times 4$ Pixel werden gemittelt, so dass die gemeinsame Kantenlänge der zusammengefassten Pixel, der $G$-Maßstab, 0, $42^{\prime \prime}$ beträgt. Abb. 5.8 zeigt das Ergebnis.

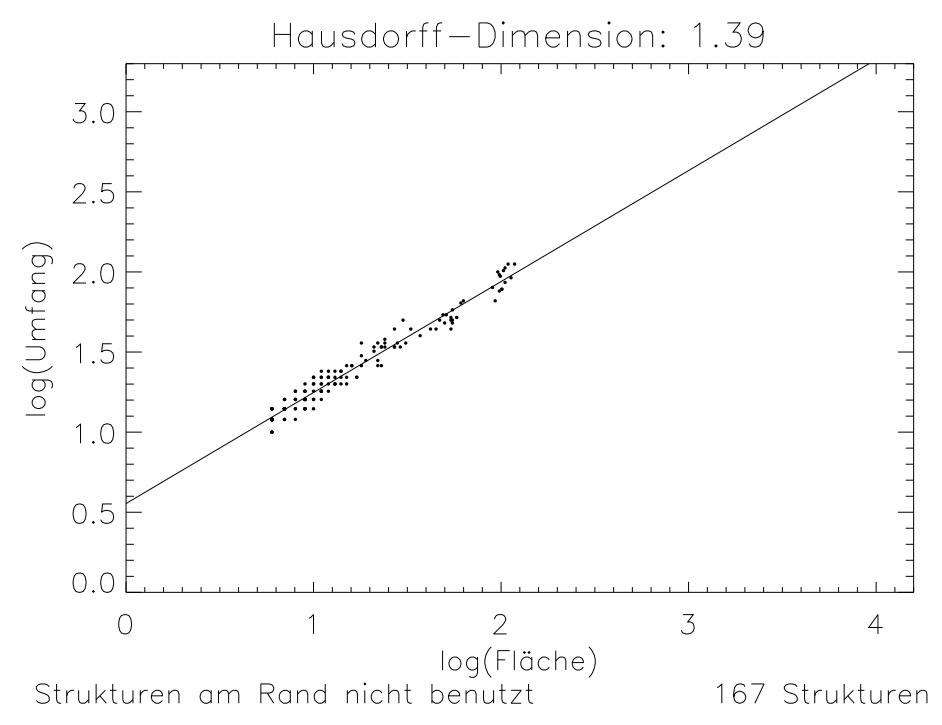

Abbildung 5.8: Bestimmung der Hausdorff-Dimension unter Benutzung eines G-Maßstabes von 4 Pixeln, entsprechend $0,42^{\prime \prime}$. 


\section{Ergebnisse}

Durch die Benutzung des größeren $G$-Maßstabes sinkt die Anzahl der für die Berechnung zur Verfügung stehenden Strukturen drastisch. Es werden 167 Strukturen gefunden, aus denen sich tatsächlich die Hausdorff-Dimension der Ränder der beobachteten Magnetfelder von mehr als 80 Gauss zu $D_{b}=1,39$ berechnet.

Um den Einfluß des $G$-Maßstabes auf die Dimensionsbestimmung genauer zu analysieren, werden die $G$-Maßstäbe systematisch untersucht. In Abb. 5.9 wird die Hausdorff-Dimension der beobachteten Magnetfelder für Maßstäbe von einem bis zu neun Pixeln gezeigt.

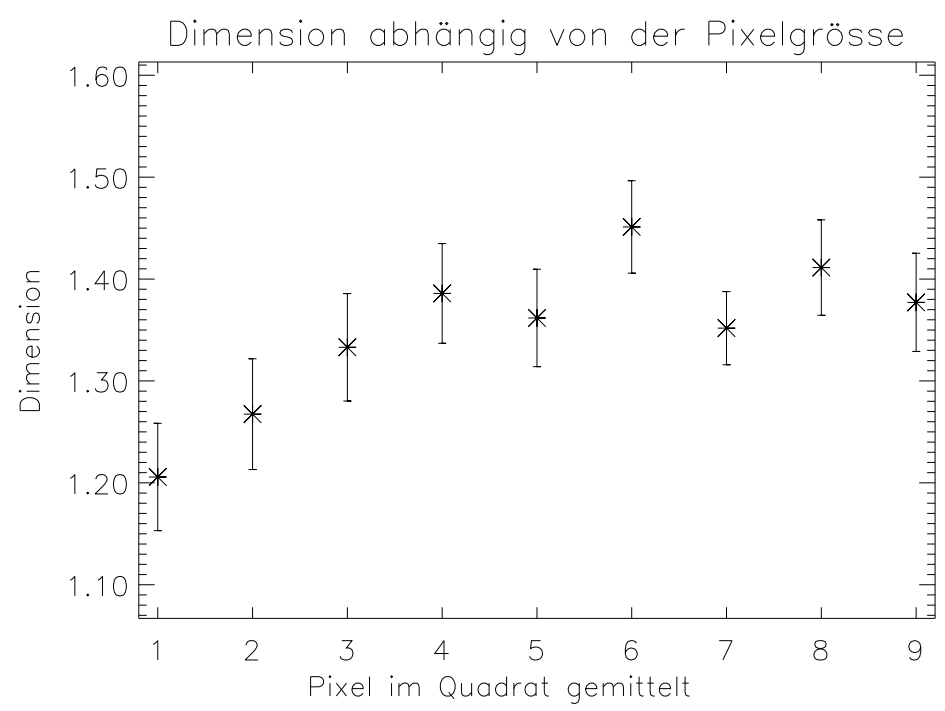

Abbildung 5.9: Abhängigkeit der für die Ränder der beobachteten Magnetfelder berechneten Hausdorff-Dimension von dem $G$-Maßstab in Pixelkantenlängen.

Für G-Maßstäbe, die kleiner als das Auflösungsvermögen der Magnetogramme von durchschnittlich $0,4^{\prime \prime}$ sind, ist ein Anstieg der Dimension mit größeren $G$-Maßstäben zu verzeichnen. Dieses erklärt sich durch die Anwendung einer übergenauen Messmethode, die die Rundungen der verbreiterten Airy-Scheibchen und nicht die echte Dimension des Randes misst.

Bei der Benutzung eines $G$-Maßstabes extrem großer Länge geschieht ein ähnlicher Fehler. Ist der benutzte $G$-Maßstab zu groß, so werden die Feinheiten im Rand kleinerer Strukturen nicht mehr erfasst. Dieses ist so lange unproblematisch, wie eine ausreichende Anzahl größerer Strukturen existiert. Da in der Praxis die maximale Ausdehnung der Strukturen jedoch durch die Größe der Detektionskamera begrenzt ist, ist diese Voraussetzung bei starker Vergrößerung des $G$-Maßstabes nicht mehr erfüllt.

Nach Mandelbrot (1997, S. 111) ist die Hausdorff-Dimension einer Struktur unabhängig von dem benutzten $G$-Maßstab. Würde diese Aussage nicht gelten, so wären Berechnungen verschiedener Beobachter, die CCD-Kameras unterschiedlicher Pixelgrößen und Teleskope unterschiedlichen Auflösungsvermögens benutzten, nicht vergleichbar, und daher eine Angabe der Größe 


\subsection{Die Hausdorff-Dimension magnetischer Strukturen}

der fraktalen Dimension sinnlos 1 . Die Unabhängigkeit der Hausdorff-Dimension von dem benutzten $G$-Maßstab wird gleichwohl von den obigen Betrachtungen nicht angetastet, sie zeigen lediglich die Grenzen der Messmethode auf.

Betrachtet man Abb. 5.9, so steigt die fraktale Dimension für die Ränder der Magnetogramme wie gerade erklärt mit zunehmendem $G$-Maßstab zunächst von $D_{b}=1,21$ auf etwa $D_{b}=1,4$ an und pendelt dann um diesen Wert. Die Schwankungen der Dimensionen nehmen für größere Maßstäbe zu, was sich aus der Anzahl der vorhandenen Strukturen erklären lässt. Abb. 5.10zeigt die der Dimensionsberechnung zu Grunde liegenden Strukturanzahlen.

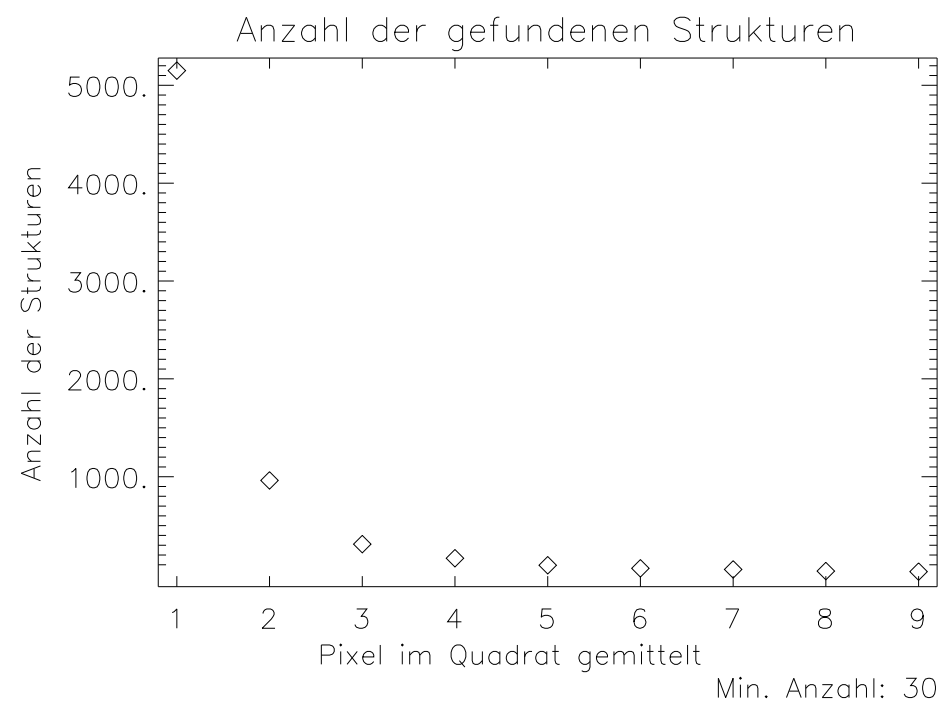

Abbildung 5.10: Abhängigkeit der für die Ränder der beobachteten Magnetfelder berechneten Hausdorff-Dimension von dem $G$-Maßstab in Pixelkantenlängen.

Ab dem $G$-Maßstab von 5 Pixellängen standen weniger als 100 Strukturen zur Verfügung, für die Berechnung der Dimension mit einem $G$-Maßstab von 9 Pixeln waren es gerade noch 30 Punkte in der Flächen-Umfangs-Relation; Entsprechend unsicherer sind die Ergebnisse der größeren Maßstäbe. Dennoch scheint sich für die größten Maßstäbe bereits ein leichter Trend zu erneut kleineren Dimensionen abzuzeichnen.

Als Resumee ergibt sich die Hausdorff-Dimension der Ränder beobachteter, magnetischer Strukturen mit Feldstärken von mehr als 80 Gauss unter Benutzung eines $G$-Maßstabes in der Größenordnung der Auflösungsgrenze von 4 Pixeln, entsprechend 0,42", zu $D_{b}=1,39$, in hervorragender Übereinstimmung mit der fraktalen Dimension, die aus den numerisch simulierten Magnetfeldern zu $D_{s}=1,40$ bestimmt wurde.

\footnotetext{
${ }^{1}$ In diesem Fall könnte lediglich eine Aussage über die Existenz einer einheitlichen fraktalen Dimension der Strukturen getroffen werden.
} 


\section{Ergebnisse}

\subsubsection{Auflösungsreduktion der Simulation}

Eine zweite Alternative zur Prüfung der Theorie, dass der Unterschied der für simulierte Magnetfelder gemessenen Dimension von $D_{s}=1,40 \mathrm{zu}$ der aus den beobachteten Magnetfeldern ursprünglich bestimmten Dimension von $D_{b}=1,21$ von dem Verhältnis des $G$-Maßstabes zur räumlichen Auflösung der Daten herrührt, ist eine künstliche Herabsetzung des Auflösungsvermögens der simulierten Daten. Auch die Ergebnisse dieser Kontrolluntersuchung sollen hier gezeigt werden.

Die Magnetfelder der Simulation werden derart in der Auflösung reduziert, dass das Verhältnis der Auflösung zum $G$-Maßstab dem der beobachteten Daten entspricht. Die experimentell gewonnenen Daten zeigen bei einer Pixelgröße von $0,105^{\prime \prime}$ eine ungefähre Auflösung von 0,42", also einen Faktor von 4. Demnach wird das Bildfeld des simulierten Magnetfeldes im Fourierraum mit einer Gaussfunktion gefaltet, deren Halbwertsbreite

$$
\mathrm{f}(k)=\sigma \cdot \sqrt{2 \pi} \cdot e^{-\frac{1}{2} k^{2} \sigma^{2}}
$$

an die der gewünschten Auflösung von 4 Pixeln entsprechende Wellenzahl von $k=0,25 \mathrm{Pixel}^{-1}$ angepasst ist; $\sigma=\sqrt{\frac{2 \cdot \ln 2}{k^{2}}}=4,7$ Pixel für diese Breite. Um die Normierung der Intensitäten nicht $\mathrm{zu}$ ändern, wird die Fouriertransformierte des Bildfeldes mit $e^{-\frac{k^{2} \sigma^{2}}{2}}$ ohne Vorfaktor multipliziert.

Die Abb. 5.11 zeigt beispielhaft die Auswirkung der künstlichen Verringerung des Auflösungsvermögens auf die simulierten Magnetfelder. Im linken oberen Teilbild ist das Original des numerisch simulierten Magnetfeldes zu sehen, rechts oben die im Auflösungsvermögen auf 4 Pixel eingeschränkte Simulation. Unten rechts ist die Maske dargestellt, in der alle Strukturen mit Magnetfeldstärken von mehr als 80 Gauss indiziert sind, links unten die mit den gleichen Indizes versehenen Umfangspixel dieser Strukturen, deren Außenseiten für die Umfangsbestimmung ausgezählt werden.

Die Hausdorff-Dimension der magnetischen Strukturen wird wie zuvor durch logarithmische Auftragung der Fläche und des Umfanges bestimmt, vgl. Abb.5.12, Die Dimension der im Verhältnis des $G$-Maßstabes zur Auflösung an die beobachteten Daten angepassten simulierten Magnetfelder ergibt sich zu $D_{s}=1,27$. Dieser Wert liegt deutlich näher an dem der ursprünglichen Dimension der beobachteten Magnetfelder von $D_{b}=1,21$, als die ursprüngliche Dimension von $D_{s}=1,40$.

Zu der Interpretation dieses Ergebnisses lässt sich zunächst feststellen, dass die Qualität der numerischen MHD-Simulationen von Vögler \& Schüssler (2003) ein Maß an Realitätsnähe erreicht hat, das eine hohe Vergleichbarkeit mit beobachteten Daten gewährleistet. Zudem zeigt sich, dass, obwohl die Größenordnungen der betrachteten Strukturen in Simulation und Beobachtung stark variieren und auch die maximalen Magnetfeldstärken beider Felder sehr unterschiedlich ausfallen, in beiden Fällen geometrische Ähnlichkeit gefunden wird, wie auch von Stenflo \& Holzreuter (2003a) proklamiert. Sogar die Größe der fraktalen Dimension scheint für beobachtete wie auch simulierte Magnetfelder im gleichen Bereich von etwa $D=1,40 \pm 0,05$ zu liegen. 
5.1 Die Hausdorff-Dimension magnetischer Strukturen
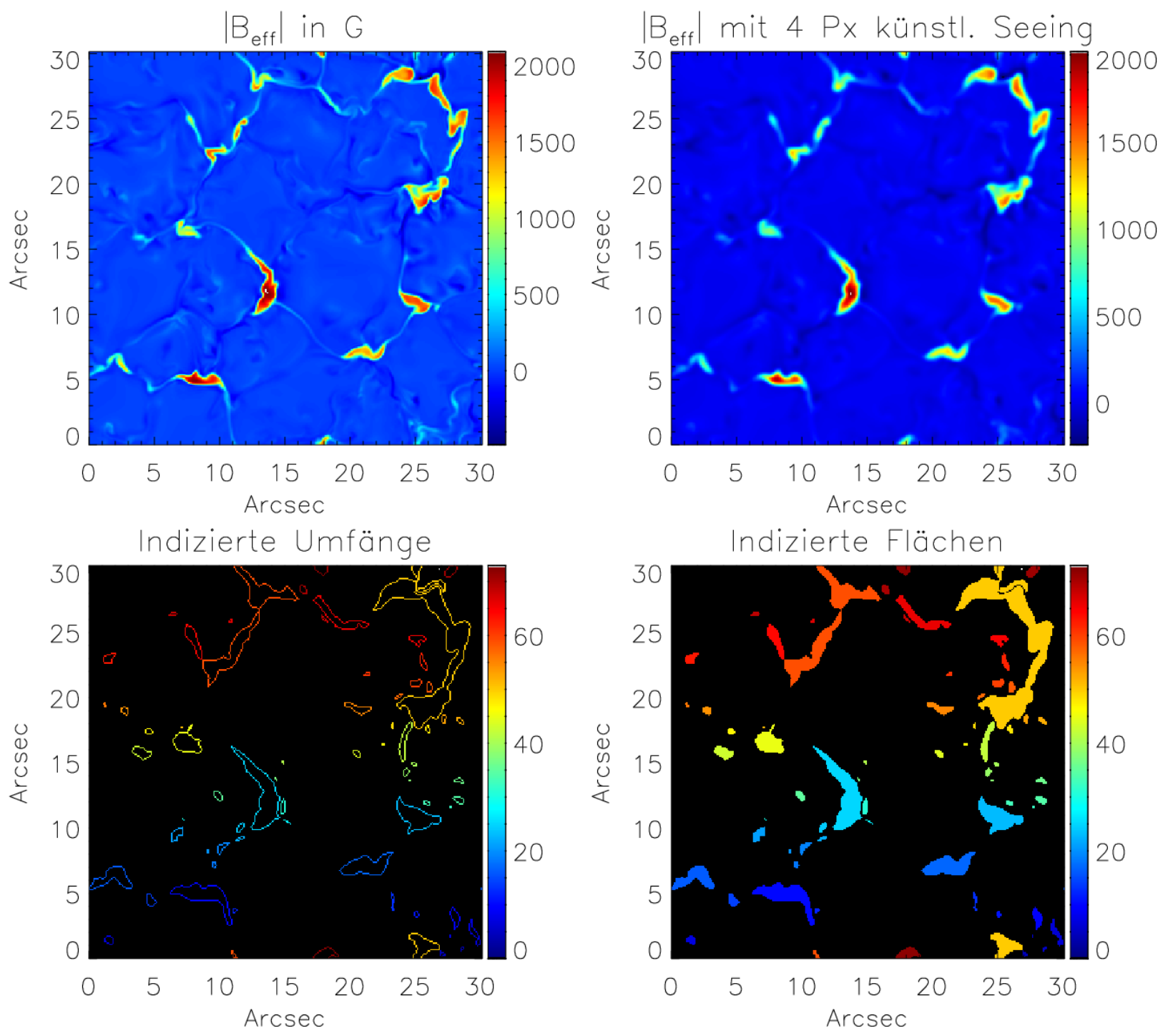

Abbildung 5.11: Oben links: Original der simulierten Magnetfeldkarte,

Oben rechts: Einschränkung des Auflösungsvermögens auf 4 Pixel, Unten rechts: Indizierung der Strukturen mit Feldstärken grösser 80 Gauss, Unten links: Randpixel der indizierten Strukturen. 


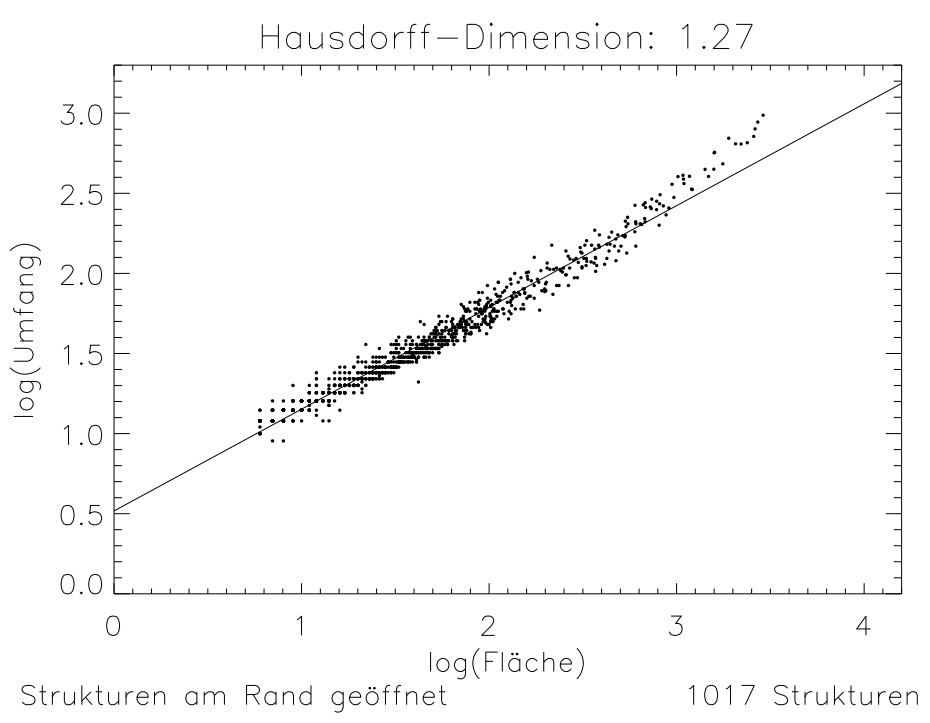

Abbildung 5.12: Bestimmung der Hausdorff-Dimension für simulierte Magnetfelder, deren Auflösung auf 4 Pixel reduziert wurde. Die Dimension von $D_{s}=1,27$ passt deutlich besser zu der ursprünglichen Dimension der beobachteten Magnetfelder von $D_{b}=1,21$.

Aufgrund der sehr unterschiedlichen Magnetfeldstärken in den beobachteten und simulierten Daten, soll an dieser Stelle noch der Einfluss des Schwellwertes der Magnetfeldstärke untersucht werden.

\subsubsection{Schwellwert der Magnetfeldstärke}

Das im vorigen Abschnitt beschriebene Ergebnis, die Ränder numerisch simulierter und beobachteter Magnetfelder besäßen eine sehr ähnliche fraktale Dimension, ist zweifelsfrei sehr befriedigend. Um die Objektivität dieses Ergebnisses besser einschätzen zu können, wird nun der Einfluss des Schwellwertes der magnetischen Feldstärke auf die Hausdorff-Dimension untersucht.

Eine Abhängigkeit der fraktalen Dimension der Ränder magnetischer Strukturen von dem Grenzwert der Feldstärke des Magnetfeldes wäre leicht einsehbar. Man erwartet, dass eine magnetische Struktur größerer Feldstärke besser gebündelt ist, als eine Struktur kleinerer Feldstärke. Diese Bündelung würde sich bei der Dimensionsbestimmung darin äußern, dass die Ränder des stärkeren Magnetfeldes glatter und somit von geringerer Dimension wären.

Die Abbildungen 5.14 und 5.13 zeigen die fraktalen Dimensionen sowohl der beobachteten, als auch der numerisch erzeugten Daten in Abhängigkeit vom Schwellwert des Magnetfeldes.

Für die simulierten Magnetfelder nimmt die Dimension der Ränder magnetischer Strukturen mit zunehmendem Schwellwert des Magnetfeldes kontinuierlich ab. Die Abnahme der Dimension 


\subsection{Die Hausdorff-Dimension magnetischer Strukturen}

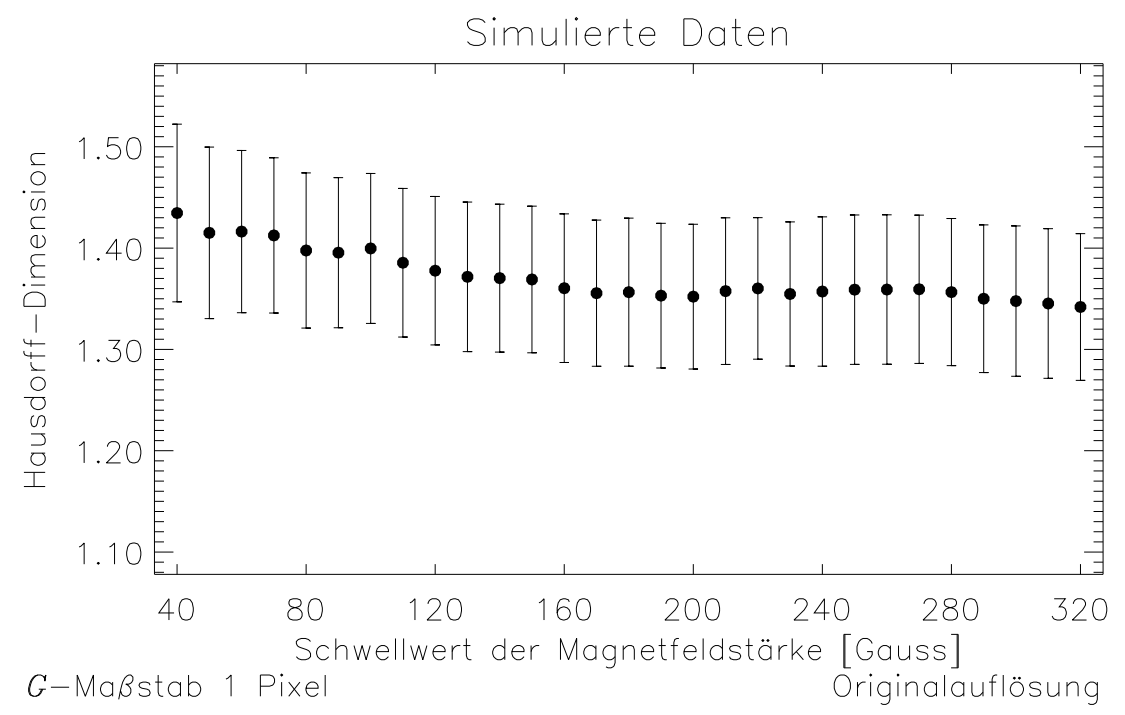

Abbildung 5.13: Abhängigkeit der fraktalen Dimension vom Grenzwert der magnetischen Feldstärke der simulierten Daten.

beträgt für den gesamten Bereich der untersuchten Magnetfeldgrenzwerte $\Delta D=0,1$ und lässt sich, wie oben beschrieben, durch die Konzentration der stärkeren Magnetfelder erklären.

In den originalen, beobachteten Magnetfeldern ist dieser Trend der Dimensionsabnahme zu größeren Feldstärken nicht zu sehen, dafür zeigt sich ein starker Abfall der gemessenen Dimension für sehr kleine Feldstärken bis etwa 80 Gauss. Die hier gemessenen, erhöhten Dimensionen könnten durch den für geringere Feldstärken stärkeren Einfluss des Rauschens verursacht sein, vgl. hierzu Kap. 5.1.11. Die Dimensionen für Magnetfeldstärken $\geq 80$ Gauss schwanken mit $\pm 0,02$ Dimensionen leicht um den Wert von $D_{b}=1,19$. Für die beobachteten Daten scheinen sich also Strukturen unterschiedlicher Magnetfeldstärke in ihrer Form nicht zu unterscheiden.

Für die Auftragung der Abb. 5.13 werden die simulierten Daten in der Originalauflösung verwendet, Abb.5.15 zeigt das Verhalten der simulierten Daten, falls die Auflösung wie in Kap. 5.1.9 beschrieben künstlich auf 4 Pixel reduziert wird, so dass für die Simulation wie für die beobachteten Originaldaten ein um den Faktor 4 zu kleiner $G$-Maßstab von 1 Pixel benutzt wird. Es zeigt sich, dass der Trend der Dimensionen, mit steigendem Schwellwert des Magnetfeldes abzunehmen, bei den in der Auflösung reduzierten, simulierten Magnetfeldern so gut wie nicht mehr vorhanden ist. Statt dessen ergibt sich hier, wie in den beobachteten Daten für mehr als 80 Gauss, ein einheitlicher Mittelwert mit geringfügigen Schwankungen.

Der Vollständigkeit halber sollte noch erwähnt werden, dass bei den simulierten Daten das ursprünglich angenommene mittlere Magnetfeld von 50 Gauss die Stärke des sich entwickelnden Magnetfeldes stark beeinflusst. Die Absolutwerte der Magnetfeldstärken sind daher in der Simulation etwas willkürlich und liegen mit 2000 Gauss deutlich höher als die der Beobachtung von 700 Gauss.

Um Beobachtung und Simulation vergleichen zu können, ist wie bereits zuvor beschrieben die 


\section{Ergebnisse}

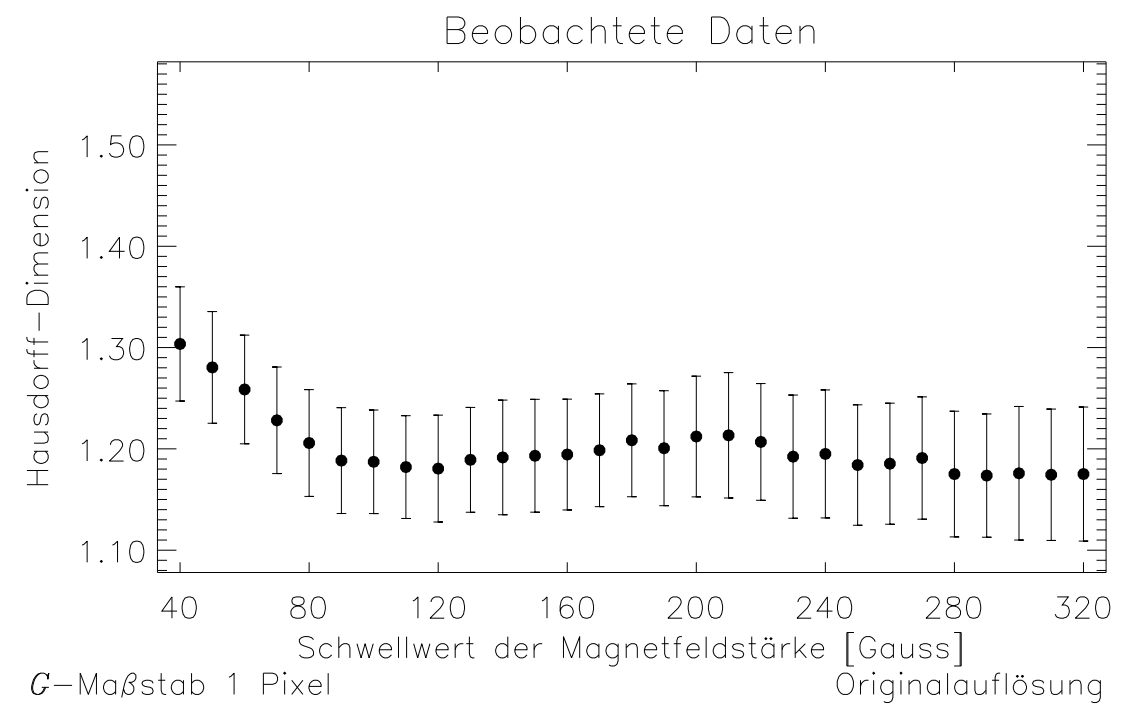

Abbildung 5.14: Abhängigkeit der fraktalen Dimension vom Grenzwert der magnetischen Feldstärke der beobachteten Daten.

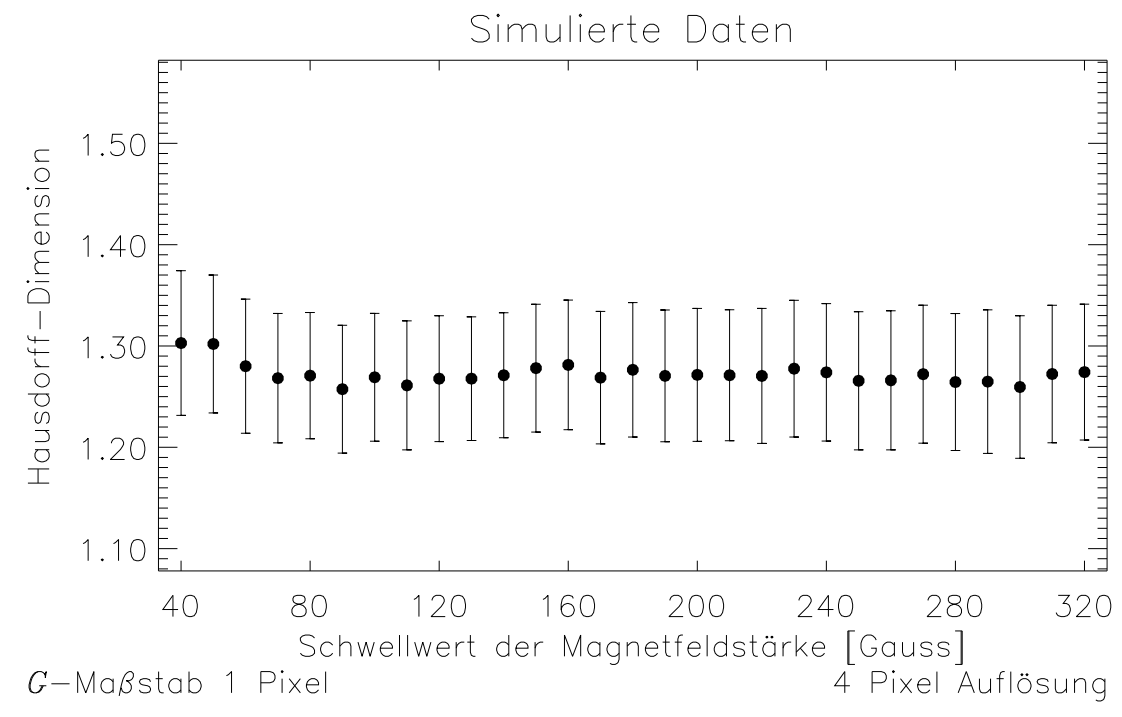

Abbildung 5.15: Abhängigkeit der fraktalen Dimension vom Grenzwert der magnetischen Feldstärke der auflösungsreduzierten, simulierten Daten. 
Anpassung der Auflösung der simulierten Daten eine Methode. Die zweite, bessere Methode jedoch ist die Benutzung des an die Auflösung angepassten $G$-Maßstabes, wie in Kap. 5.1.8 erklärt. Abb. 5.16 zeigt nun die Änderung der Dimension mit dem Magnetfeldschwellwert unter Benutzung des an die Auflösung von $0,42^{\prime \prime}$ angepassten $G$-Maßstabes.

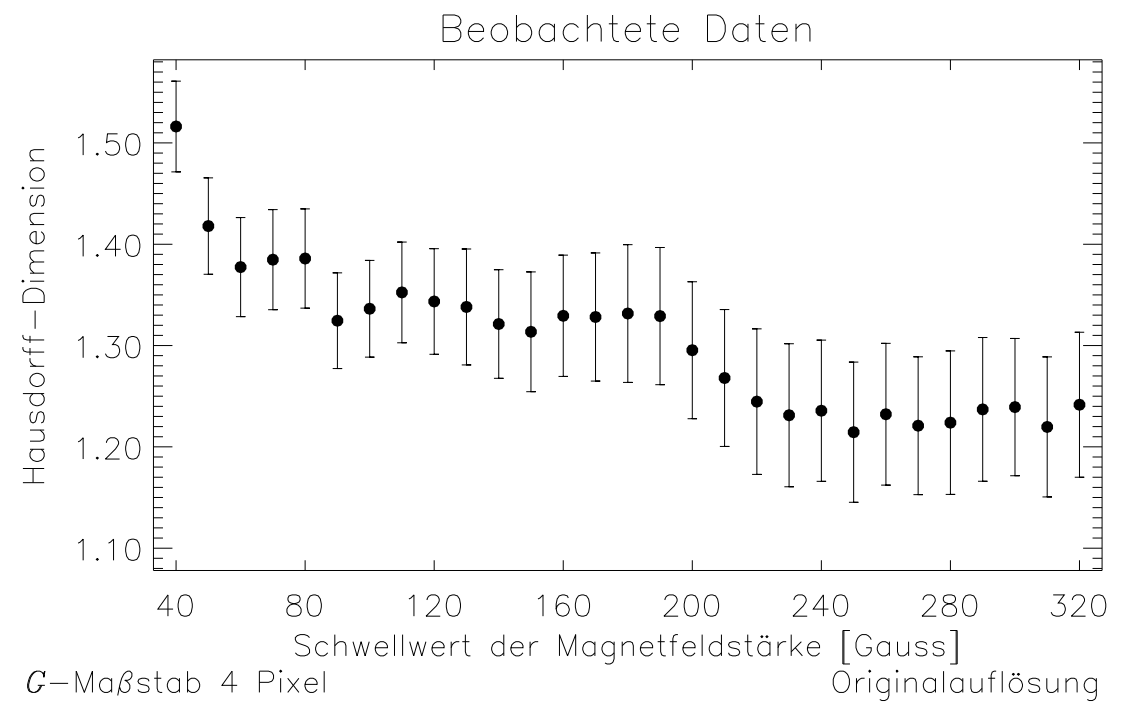

Abbildung 5.16: Abhängigkeit der fraktalen Dimension vom Grenzwert der magnetischen Feldstärke für die beobachteten Daten bei Benutzung eines der Auflösung entsprechenden $G$-Maßstabes von 4 Pixeln.

In dieser Auftragung wird die ursprüngliche Erwartung einer abnehmenden Dimension der Ränder magnetischer Strukturen mit zunehmendem Schwellenwert für das Magnetfeld bestätigt. Die deutliche Steigung der Abhängigkeit lässt sich zum einen dadurch erklären, dass bei tiefer liegenden Grenzwerten einzelne magnetische Strukturen zu einer größeren Struktur mit komplizierterem Rand zusammenwachsen, zum anderen wäre es auch gut möglich, dass die schwächeren Magnetfelder tatsächlich stärker "ausgefranst" sind als die starken Magnetfelder.

Vor allem die Sprünge im linken Teil der Auftragung stützen die erste These, denn gerade sie deuten darauf hin, dass bei einer geringeren Feldstärke komplizierte Strukturen mit hoher fraktaler Dimension gefunden werden, die für einen nur 10 Gauss höheren Schwellwert in mehrere einfachere Strukturen mit geringerer Dimension zerfallen.

Die Abnahme der Dimension mit der Magnetfeldstärke ist in Abb.5.16 sehr deutlich, doch auch in der Simulation in Originalauflösung, Abb. 5.13, war der Trend zu sehen.

Da die Messung der Hausdorff-Dimension mit einem Maßstab, der kleiner als die Auflösung des Bildes ist, nicht zu korrekten Ergebnissen führen kann, kann davon ausgegangen werden, dass Abb. 5.16 am ehesten die wahren Begebenheiten widerspiegelt. Deshalb sollen auch für diese Berechnungen noch einmal die zu Grunde liegenden Anzahlen in Abb. 5.17 dargestellt werden.

Bereits ab 120 Gauss variiert die Anzahl der Strukturen nur noch sehr wenig, so dass für alle 


\section{Ergebnisse}

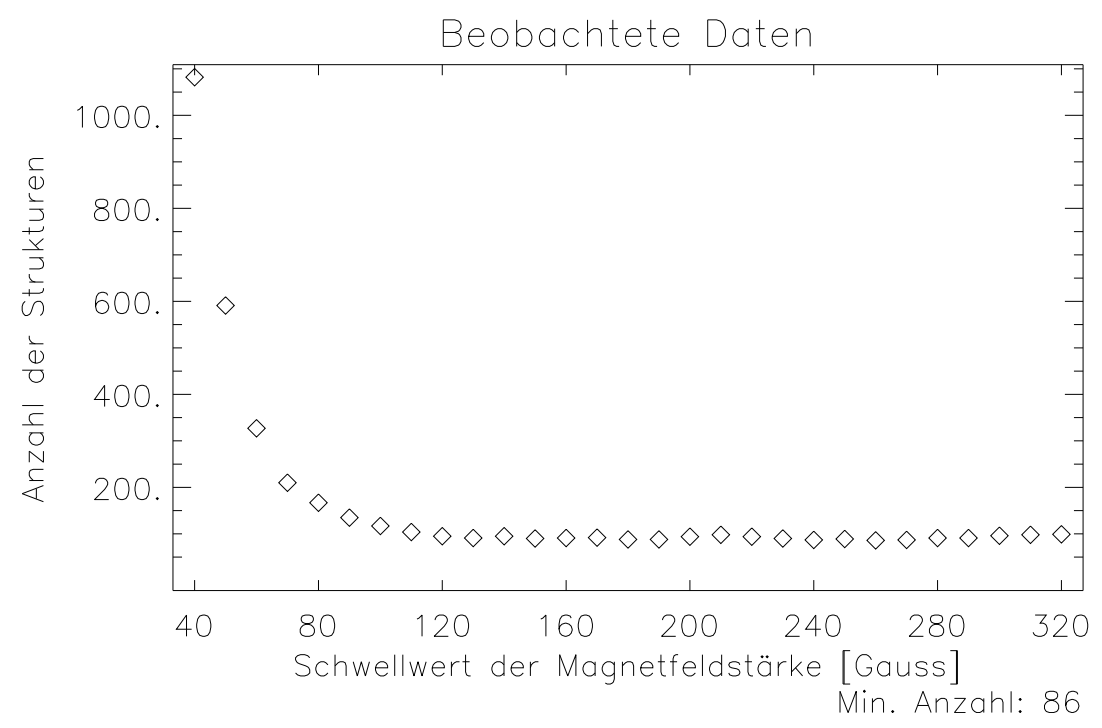

Abbildung 5.17: Anzahl der Strukturen, die zu den Schwellwerten der magnetischen Feldstärke unter Benutzung eines der Auflösung entsprechenden $G$-Maßstabes von 4 Pixeln in den beobachteten Daten gefunden wurden.

Dimensionen mit höheren Magnetfeldgrenzen die gleichen Strukturen herangezogen werden. Andererseits muss sicherlich auch zugestanden werden, dass diese Anzahl unter 100 liegt. Für die drei anderen Auftragungen dieses Kapitels standen bis zum höchsten Magnetfeldschwellwert über 200 Strukturen zur Verfügung.

\subsubsection{Rauschen}

Da die Standardabweichung der mit der COG-Methode gemessenen Sichtlinienkomponente des Magnetfeldes $\sigma=40$ Gauss beträgt, wird der Schwellwert des Magnetfeldes für die Bestimmung der Hausdorff-Dimension der magnetischen Strukturen auf 80 Gauss festgesetzt. Für die Untersuchung der Abhängigkeit der Dimension von dem magnetischen Schwellwert werden jedoch auch kleine Grenzwerte ab 40 Gauss betrachtet, obwohl diese durchaus rauschdominiert sein könnten.

Um die Abhängigkeit der Hausdorff-Dimension von dem Rauschen der Daten abschätzen zu können, wird für Abb. 5.18 bei einem festen Schwellwert von 80 Gauss ein gaussverteiltes Rauschen von 10 bis 140 Gauss addiert, bevor die Hausdorff-Dimension berechnet wird. Hierfür wird, wie in Kap. 5.1.8 beschrieben, ein $G$-Maßstab von vier Pixeln auf die Beobachtungsdaten angewandt.

Die ohne künstliches Rauschen berechnete Hausdorff-Dimension beträgt $D_{b}=1,39$. Sofern das addierte Rauschen unter dem Schwellwert des Magnetfeldes liegt, tritt keine signifikante Änderung der Dimension ein. Erst beim Überschreiten des Schwellwertes von 80 Gauss steigt 


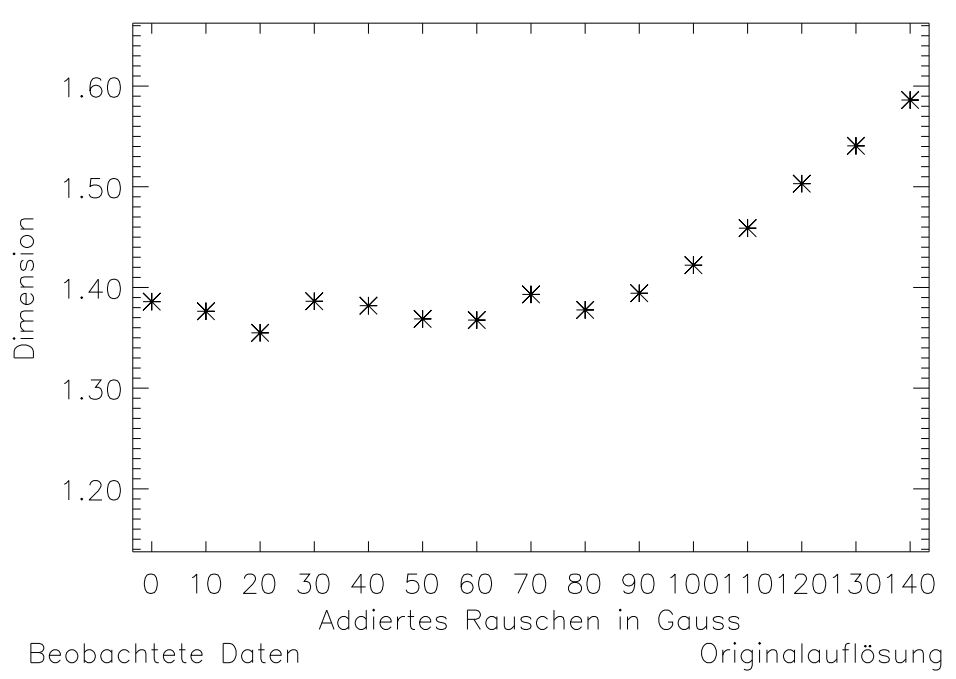

Abbildung 5.18: Der Einfluss künstlichen Rauschens auf die Berechnung der HausdorffDimension, basierend auf der Dimension der beobachteten Magnetfelder $D_{b}=$ 1,39 mit einem $G$-Maßstab von 4 Pixeln.

die berechnete Hausdorff-Dimension durch das zusätzliche Rauschen an. Hieraus kann geschlossen werden, dass die erzielten Ergebnisse vom Rauschen nicht sichtbar beeinflusst werden.

In den folgenden Abschnitten werden weitere Untersuchungen zur Abschätzung eventueller Fehler bei der Bestimmung der Hausdorff-Dimension gezeigt. Hierfür wird wie zu Beginn des Kapitels, sofern nichts anderes angegeben ist, ein Schwellwert des Magnetfeldes von 80 Gauss und ein $G$-Maßstab von einem Pixel benutzt. Die Ergebnisse müssen daher mit der ursprünglichen Berechnung der Hausdorff-Dimension der beobachteten Magnetfelder von $D_{b}=1,21$ verglichen werden.

\subsubsection{Seeingparameter}

Auch die Abhängigkeit der Hausdorff-Dimension von dem Seeing des betrachteten Scans soll untersucht werden.

Im Gegensatz zu den Ergebnissen der vorigen Kapitel werden die Strukturen der 120 beobachteten Scans für diesen Vergleich nicht gemeinsam ausgewertet. Anstelle dessen wird für jeden Scan einzeln eine fraktale Dimension berechnet und gegen den Fried-Parameter $r_{0}$ dieses Scans aufgetragen. Abb.5.19 demonstriert die Streuung der sich ergebenden Dimensionen.

Gerade weil hier Hausdorff-Dimensionen aus einzelnen Scans bestimmt werden, ist die Anzahl der gefundenen Strukturen von größtem Interesse. Einerseits kann ein einzelner Scan nicht mehr als 100 Strukturen enthalten, andererseits ist eine Dimension, die aus drei Punkten in der Flächen-Umfangs-Relation bestimmt wird, nicht gerade zuverlässig. In Abb. 5.20 ist die An- 


\section{Ergebnisse}

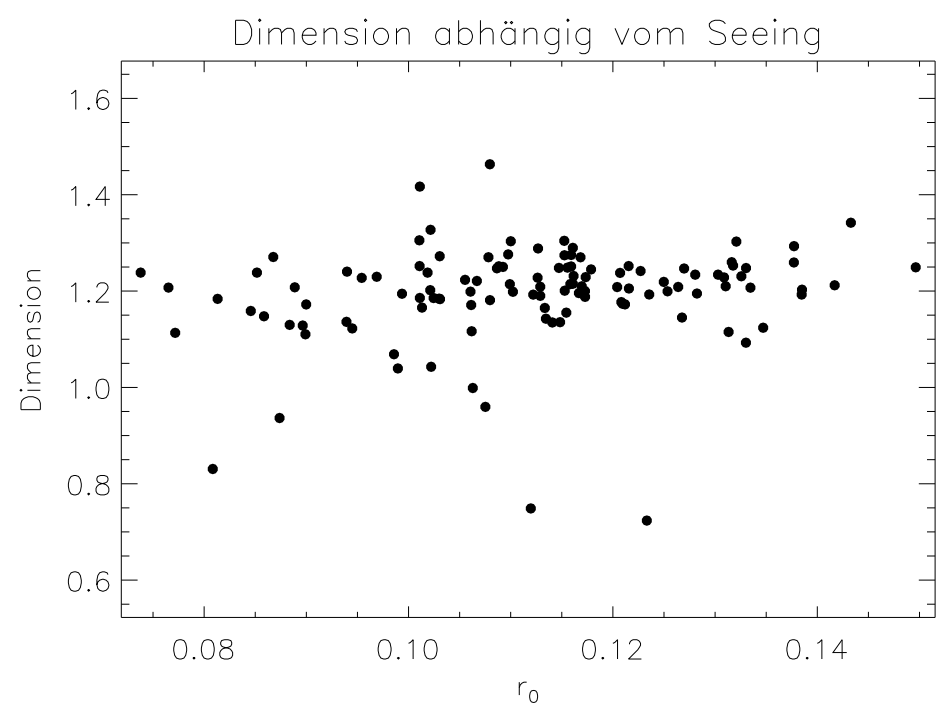

Abbildung 5.19: Abhängigkeit der Hausdorff-Dimension vom Fried-Parameter $r_{o}$.

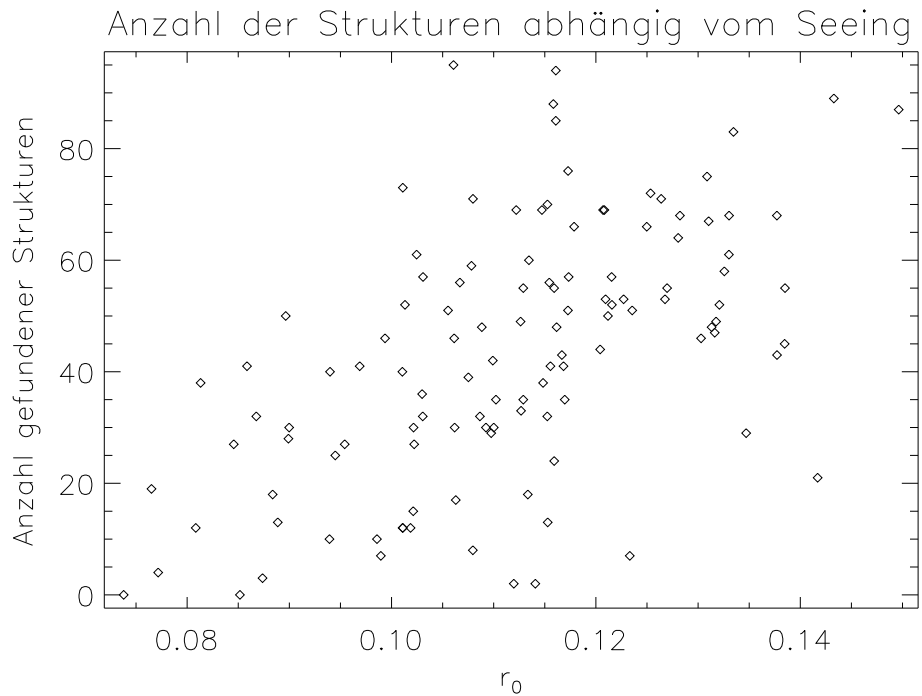

Abbildung 5.20: Abhängigkeit der Anzahl der auswertbaren Strukturen vom Seeing. 


\subsection{Die Hausdorff-Dimension magnetischer Strukturen}

zahl der Strukturen in Abhängigkeit von dem Fried-Parameter dargestellt. In Scans mit besserem Seeing werden wie erwartet durchschnittlich mehr Strukturen detektiert als in Scans mit schlechterem Seeing, doch auch Scans mit einem mittleren Fried-Parameter von $r_{0}=10-12 \mathrm{~cm}$ enthalten gelegentlich weniger als 20 Strukturen mit einer Feldstärke von mehr als 80 Gauss.

Um für die berechneten Dimensionen ein gewisses, wenn auch geringes Maß an Qualität zu garantieren, sollen aus Abb. 5.19 alle Dimensionen, die aus weniger als 20 Strukturen berechnet wurden, entfernt werden. Das Ergebnis, Abb. 5.21, zeigt ein deutlich homogeneres Feld. Die Streuung der Dimensionen ist zurückgegangen, sie schwanken mit einer Abweichung von $\Delta D=$ 0,1 um einen Mittelwert von 1,2. Die Abweichungen der einzelnen Dimensionen um 0,1 sollten nicht als Genauigkeit der in Kap. 5.1.6 und 5.1.8 angegebenen Dimensionen betrachtet werden, da diese Dimensionen auf wesentlich größeren Stichproben beruhen.

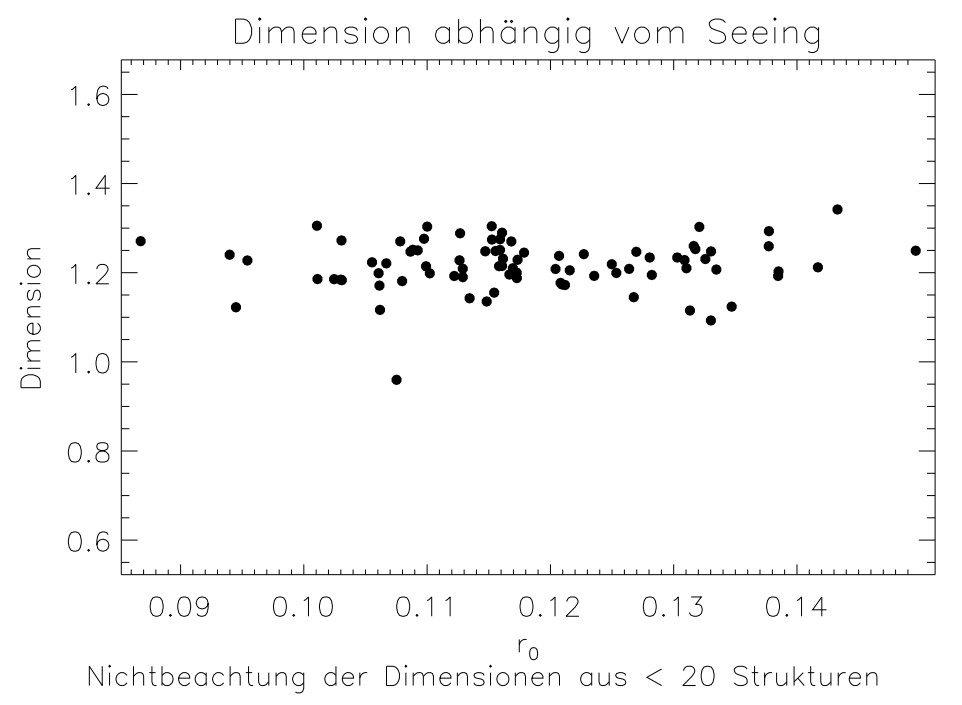

Abbildung 5.21: Abhängigkeit der Dimension vom Fried-Parameter $r_{o}$, unter Nichtberücksichtigung von Dimensionen, die aus weniger als 20 Strukturen berechnet wurden.

Die wichtigste Aussage der Abb. 5.21 ist jene, dass die fraktalen Dimensionen der einzelnen Scans nicht von dem Seeing-Parameter $r_{0}$ abhängen. Sofern ein Mindestmaß an Qualität in den Rohdaten gegeben ist, liefert die Speckle-Rekonstruktion offensichtlich Magnetfelder konsistenter Formen. Es ist kein Anstieg der Dimensionen bei besserem Seeing zu verzeichnen, und auch die Streuung der Dimensionen hängt vielmehr von der Anzahl der gefundenen Strukturen als von dem Fried-Parameter ab. Die Auftragung der Dimensionen gegen die Anzahl der Strukturen, Abb. 5.22, zeigt dieses.

Es ist eindeutig, dass sich die extremen Dimensionen von etwa 0,7 oder 1,5 aus sehr wenigen Strukturen in der Flächen-Umfangs-Relation berechnen. Ab einer Anzahl von etwa 20 oder 40 Strukturen verringert sich die Abweichung der Dimensionen auffallend. 


\section{Ergebnisse}

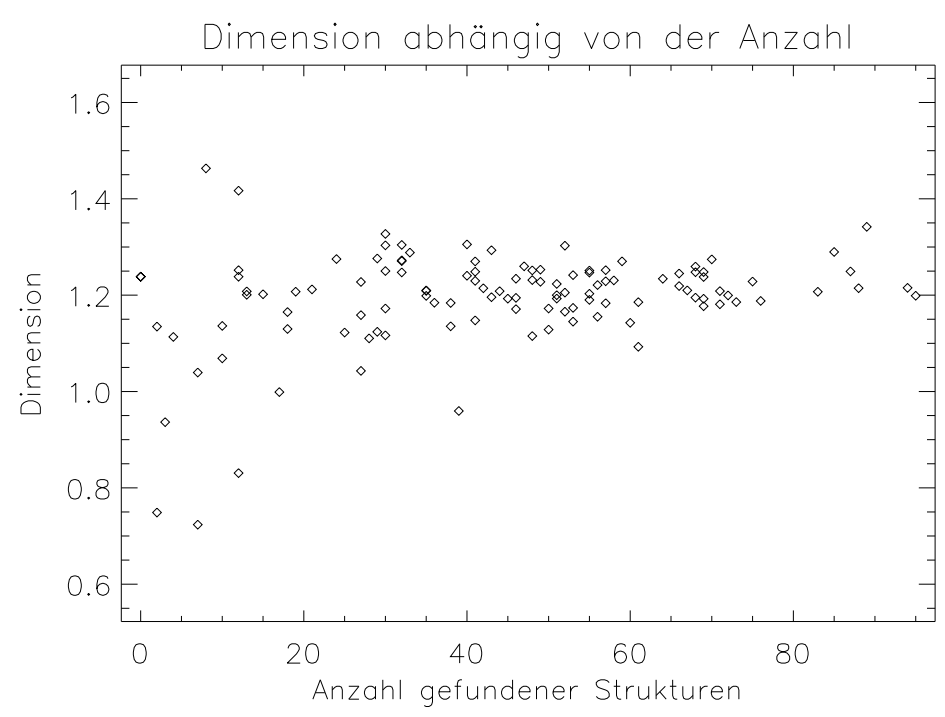

Abbildung 5.22: Abhängigkeit der Dimension von der Anzahl der für die Berechnung zur Verfügung stehenden Punkte.

\subsubsection{Behandlung des Bildrandes}

Der Vollständigkeit halber soll erwähnt werden, dass verschiedene Versuche zur Behandlung des Bildfeldrandes unternommen wurden. In allen obigen Ergebnissen werden Strukturen, die den Bildfeldrand berühren, nicht berücksichtigt. Alternativ könnten Strukturen, die den Rand berühren, als abgeschlossen betrachtet werden, d.h. sie enden mit dem Bildfeldrand, so dass dieser mit in die Bestimmung des Umfanges eingeht, oder die Strukturen am Bildfeldrand könnten als geöffnet behandelt werden, wobei die zum Bildfeldrand zeigenden Seiten der Umfangspixel nicht gezählt werden, so dass die Struktur über den Bildfeldrand hinausragt.

Da bei geöffnetem Bildfeldrand nicht festgestellt werden kann in welchem Verhältnis Fläche und Umfang fehlender Pixel unterschlagen werden, kann diese Methode nicht zu sicheren Ergebnissen führen. Gleiches gilt für die Benutzung eines geschlossenen Bildfeldrandes, denn es ist extrem unwahrscheinlich, dass die Ränder der betrachteten Strukturen tatsächlich auf dem Bildfeldrand liegen.

Strukturen, die den Rand des Bildfeldes berühren, werden daher in allen dargestellten Ergebnisse ignoriert. Dies reduziert zwar die Stichprobe, schließt aber eine mögliche Fehlerquelle aus.

Es soll dennoch kurz erwähnt werden, dass auch die Anwendung eines geöffneten oder geschlossenen Bildfeldrandes nicht zu deutlich anderen Ergebnissen für die Bestimmung der HausdorffDimension führen. Die Variation beträgt für die beobachteten Daten bei einem Schwellenwert von 80 Gauss nur $\Delta D=0,02$. 


\subsubsection{Große Strukturen}

Im Blickfeld vieler Arbeiten zur Hausdorff-Dimension (z.B. Hirzberger et al. (1997); Schrijver et al. (1992); Balke et al. (1993)) steht die Frage nach der Änderung der Dimension für größere Strukturen. Es wird untersucht, ob räumlich ausgedehntere Strukturen größere Hausdorff-Dimensionen besitzen als kleinere. Diese Frage ist deshalb von entscheidendem Interesse, weil sie die grundlegende Frage nach der Selbstähnlichkeit der Strukturen beinhaltet. In den Veröffentlichungen zur fraktalen Dimension der Ränder von Granulen werden diese häufig in zwei Größenklassen eingeteilt, für die zwei unterschiedliche Dimensionen bestimmt werden. Roudier \& Muller (1987) u.a. erhalten hierbei für die Ränder ihrer größeren Granulen sogar Hausdorff-Dimensionen von über 2,0 .

Da auch in den vorliegenden Daten ein leichter Trend zu größeren Dimensionen für größere Strukturen vorliegt, soll eine kurze Untersuchung vorgestellt werden.

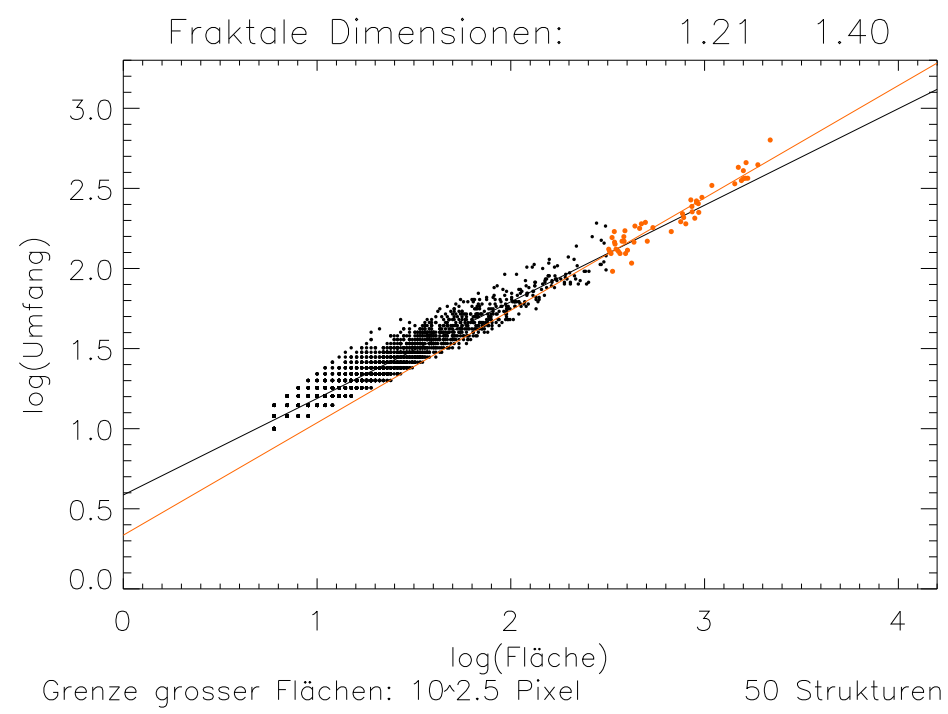

Abbildung 5.23: Bestimmung einer zweiten Hausdorff Dimension für Strukturen mit $\log ($ Fläche $) \geq 2.5$.

In Abb. 5.23 ist noch einmal die ursprüngliche Flächen-Umfangs-Relation der beobachteten Daten dargestellt. Wie in Kap. 5.1.6 werden alle magnetischen Strukturen von mehr als 80 Gauss Feldstärke unter Benutzung eines $G$-Maßstabes von einem Pixel berücksichtigt. Ab einem Wert von $\log ($ Fläche $) \geq 2,5$ ist die Tendenz zu einer höheren Dimension bemerkbar, daher wird für diese Punkte eine zweite Dimension berechnet, die sich zu $D_{b, b i g}=1,40$ ergibt. Für diese Dimensionsbestimmung standen 50 Strukturen mit Flächen $>316$ Pixel zur Verfügung. Eine maximale Dimension für die großen Strukturen erhält man, indem die Grenze zur Bestimmung der zweiten Dimension bei $\log ($ Fläche $) \geq 2,8$ gewählt wird, weil sich gerade dieser Größe ein vermutlich zufälliger Sprung zu kleineren Umfängen befindet. Aus den verbleibenden 25 Strukturen, die in ihrer Größe diese Grenze von 630 Pixeln überschreiten, kann die extrem hohe fraktale Dimen- 


\section{Ergebnisse}

sion von $D_{b, b i g}=1,82$ bestimmt werden. Während dieses Ergebnis dem Zufall zugeschrieben wird, kann die Dimension von $D_{b, b i g}=1,40$ als real angesehen werden. Sie läßt sich vermutlich dadurch erklären, dass großflächige Strukturen die Tendenz haben aus mehreren Unterstrukturen zusammenzuhängen, während kleinere Strukturen mit größerer Wahrscheinlichkeit einzelne Strukturen sind.

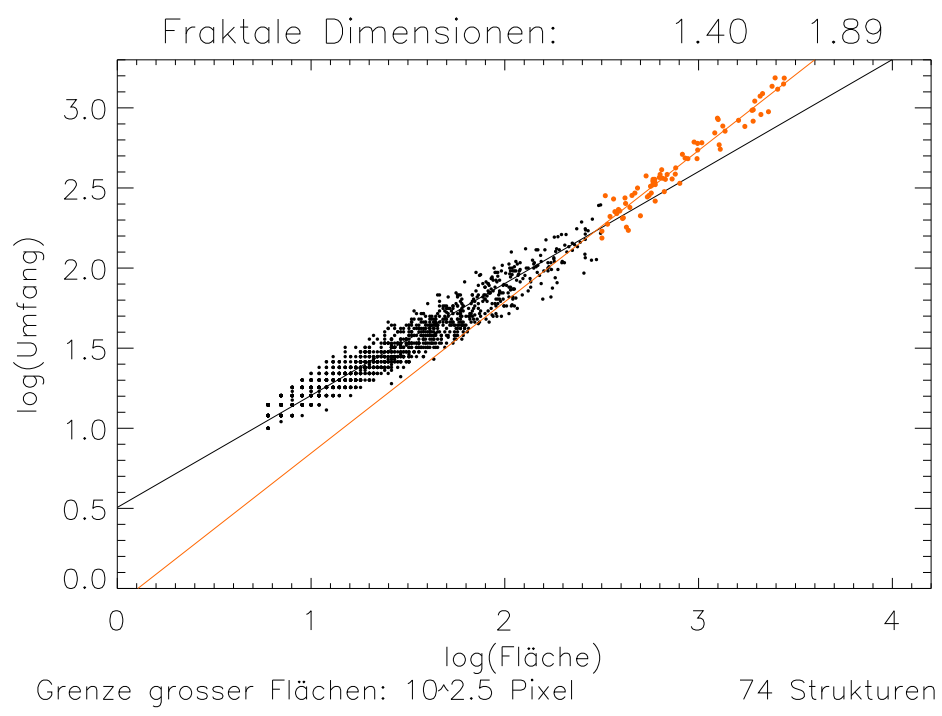

Abbildung 5.24: Bestimmung einer zweiten Hausdorff Dimension für Strukturen mit $\log ($ Fläche $) \geq 2.5$ aus den simulierten Daten.

Es wäre noch anzumerken, dass sich der Trend zu größeren Dimensionen für größere Strukturen auch in den simulierten Daten zeigt. In den numerisch simulierten Magnetfeldern ist bereits bei $\log ($ Fläche $) \geq 2,5$ eine starke Steigerung der Dimension zu sehen, vgl. Abb. 5.24. Aus 74 Strukturen von mehr als 316 Pixeln ergibt sich die Dimension zu $D_{s, b i g}=1,89$.

Interessant ist hierbei, dass sowohl die Beobachtungen als auch die Simulationen eine Vergrößerung der Dimension für Strukturen ab $\log$ (Fläche) $\geq 2,5$ erleben, obwohl diese Flächengrenzwerte wegen der unterschiedlichen Pixelgrößen von $21 \mathrm{~km}$ für die Simulation und $73 \mathrm{~km}$ für die Beobachtung in keinem Zusammenhang stehen.

\subsubsection{Kreise}

Um einen letzten Versuch zur Absicherung der Ergebnisse zu unternehmen, werden auch Datenfelder, die ausschließlich ausgefüllte Kreise als Strukturen enthalten, untersucht. Pro "Aufnahme" werden etwa 20 Kreise mit Radien von einem bis 30 Pixeln auf $288 \times 288$ Pixel großen Feldern zufällig verteilt. Aus 10 dieser Aufnahmen wird die Hausdorff-Dimension bestimmt, die sich nach Abb. 5.25 zu $D_{\text {kreis }}=1,01$ ergibt und der Erwartung von $D=1,00$ entspricht. 


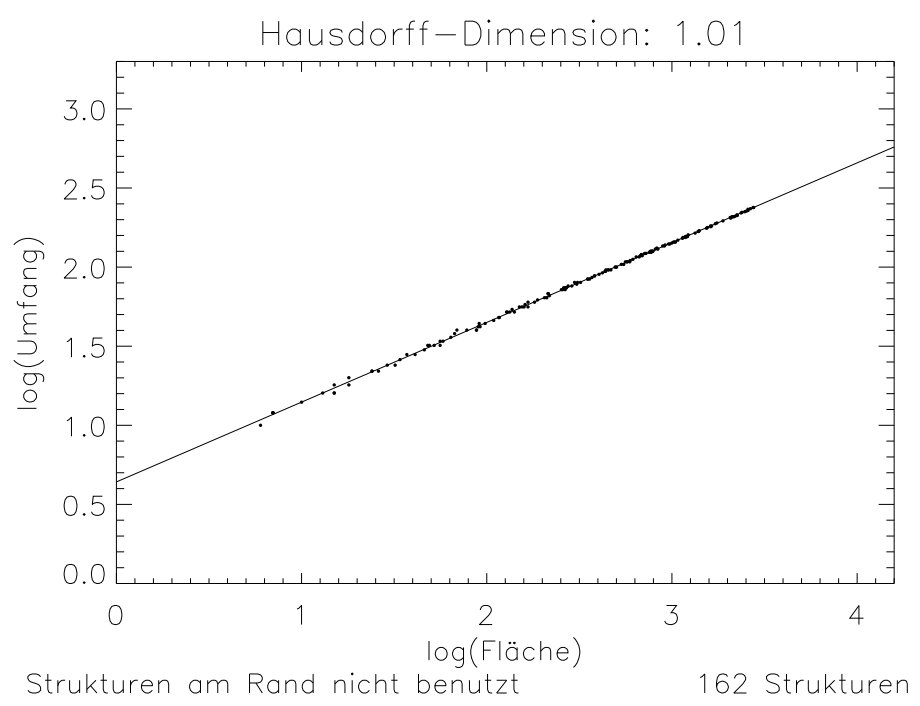

Abbildung 5.25: Hausdorff-Dimension zufällig verteilter Kreise mit Radien bis 30 Pixel.

\subsubsection{Zusammenfassung}

Die oben dargestellte Analyse der Hausdorff-Dimension der Ränder magnetischer Strukturen lässt sich wie folgt zusammenfassen:

1. Magnetische Strukturen sind selbstähnlich, denn für einen festen Schwellwert des Magnetfeldes wird über mehrere Größenskalen eine einheitliche fraktale Dimension gefunden, vgl. Kap. 5.1.6 und 5.1.8.

2. Die beobachten Magnetfelder besitzen für einen Schwellwert der Magnetfeldstärke von 80 Gauss unter Benutzung eines an die Auflösung der Magnetogramme von 0,42" angepassten $G$-Maßstabes eine Hausdorff-Dimension von $D_{b}=1,39 \pm 0,05$, siehe auch Abb. 5.8.

3. Für 50 sehr große Strukturen wird eine größere Hausdorff-Dimension gefunden, eine Berechnung mit korrektem $G$-Maßstab ist jedoch wegen der dann noch geringeren Stichprobe nicht möglich, vgl. Kap. 5.1.14.

4. Für sehr kleine Strukturen ist kein Ende der Selbstähnlichkeit abzusehen. Man kann daher vermuten, dass die Ränder noch kleinskaliger magnetischer Strukturen, könnte man sie auflösen, ebenfalls die gleiche fraktale Dimension besäßen. Wie in Stenflo \& Holzreuter (2003a) beschrieben, würden diese Strukturen vergrößert ebenso aussehen wie die Strukturen, die wir bereits auflösen können.

5. Mit der Variation der Grenze der Magnetfeldstärke zeigt sich vor allem bei den beobachteten Daten doch eine Änderung der berechneten Hausdorff-Dimensionen, siehe Abb. 5.16 . 


\section{Ergebnisse}

So haben die Ränder stärkerer Magnetfelder in Übereinstimmung mit den Ergebnissen von Meunier (1999) kleinere fraktale Dimensionen als die Ränder der schwachen Magnetfelder. Dies erklärt sich daraus, dass Strukturen mit einem stärkeren Magnetfeld besser gebündelt sind als Strukturen mit schwächerem Magnetfeld. Der Unterschied der fraktalen Dimensionen für die betrachteten Magnetfeldstärken von 40 Gauss bis 320 Gauss beträgt $\Delta D=0,2$.

6. Der Vergleich der Magnetfeldränder numerisch simulierter und beobachteter Daten verläuft verblüffend gut. Unter der Voraussetzung, dass das Verhältnis des $G$-Maßstabes zu der Auflösung der Daten für simulierte und beobachtete Magnetfelder gleich ist, werden in Abb. 5.8 und Abb. 5.7 für einen Schwellwert von 80 Gauss sehr ähnliche fraktale Dimensionen gefunden.

7. Auch der Trend der geringeren Hausdorff-Dimensionen für höhere Schwellwerte des Magnetfeldes lässt sich in den simulierten Daten finden, jedoch weit weniger ausgeprägt als in den beobachteten Daten, siehe Abb. 5.13.

8. Der Einfluss mehrerer möglicher Störungen wird separat untersucht. So ergibt sich, dass die Hausdorff-Dimension keine Abhängigkeit vom Seeing aufweist, siehe Abb. 5.21. Auch existiert, wie in Abb. 5.5 zu sehen, keine feststellbare Mitte-Rand-Variation der fraktalen Dimensionen. Die Addition eines gaussverteilten Rauschfeldes von 60 Gauss liefert keine signifikante Abweichung der Dimension, vgl. Abb. 5.18. Selbst die bewusste Fehlbehandlung des Bildfeldrandes als abgeschlossen oder geöffnet, vgl. Kap. 5.1.13, ergibt nur eine Abweichung der Dimension von $\Delta D=0,02$. Und ein Test mit Datenfeldern, die ausschließlich Kreise enthalten, siehe Abb. 5.25, liefert wie erwartet die Hausdorff-Dimension $D_{\text {kreis }}=1,01$, d.h. sehr nahe $D=1,00$. 


\subsection{Energiezufuhr durch Umstrukturierung der Magnetfelder}

Durch die horizontale Auslenkung eines magnetischen Gebietes, vgl. Abb. 5.26, angestoßen durch die Bewegung der Granulation, überträgt letztere Energie auf das Magnetfeld. Im Folgenden soll eine Abschätzung der Größenordnung der so transportierten Energie vorgenommen werden und somit die Frage nach der Heizung der höheren Atmosphärenschichten beleuchtet werden.

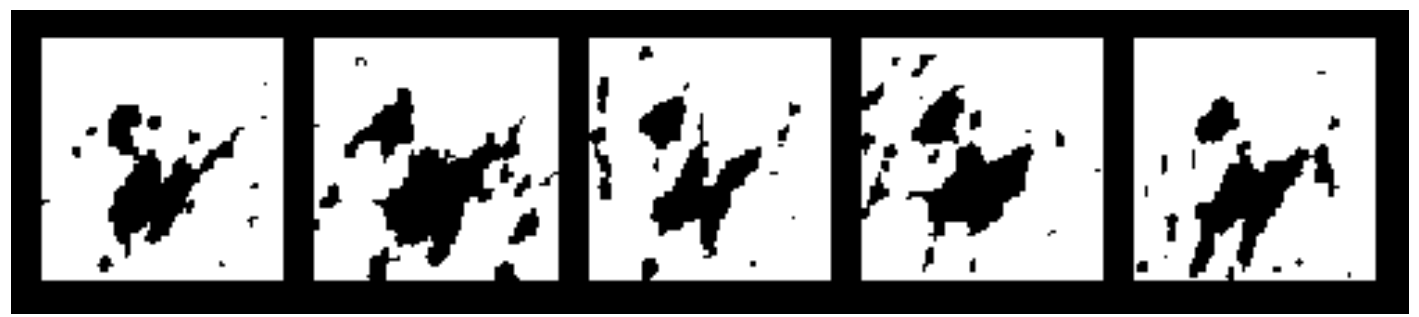

Abbildung 5.26: Änderung magnetischer Strukturen in $50 \mathrm{~s}$ Schritten. Binäre Masken mit Schwellwert von 120 Gauss. Größe des Ausschnitts $7^{\prime \prime} \times 7^{\prime \prime}$, Serie 44.

\subsubsection{Motivation}

Der Energietransport aus den unteren Schichten der Sonnenatmosphäre in hohe Schichten - Chromosphäre, Übergangszone und Korona - ist ein Thema von größtem Interesse, da nicht eindeutig geklärt ist, wie die Heizung dieser oberen Schichten funktioniert. Vertikale Wellen magnetischer Flussröhren, die mit ihrer Ausbreitung Energie transportieren, werden in diesem Zusammenhang oft diskutiert. Es gibt drei verschiedene Wellen-Moden, longitudinale Dichtewellen (engl. saussage mode), torsionale Rotationswellen (Alfvén Wellen) und transversale Scherwellen (engl. kink mode). Der größte Anteil am Energietransport wird hierbei den transversalen Wellen zugesprochen. Diese Wellen entstehen durch horizontale Stöße, die sich in Form von transversalen Verschiebungswellen in der Sonnenatmosphäre fortpflanzen und so ihre Energie in die Chromosphäre oder Korona tragen. Eine genaue Bilanz liegt für diesen Energietransport nicht vor, es existieren jedoch Abschätzungen der Größenordnung (siehe unten).

Ein weiterer Heizungsmechanismus besteht aus der Zuführung magnetischer Energie durch Aufbau von komplexen Magnetfeldtopologien bei Bewegung der Fußpunkte in der subphotosphärischen Konvektion. Durch Umordnung der Magnetfelder wird hier über Ohm'sche Verluste die magnetische Energie in Wärme umgesetzt. (Zu Heizungsmechanismen siehe Narain \& Ulmschneider 1996).

In der Balmer- $\alpha$-Linie des Wasserstoffs $(\mathrm{H} \alpha)$ beobachtete Strukturen der oberen Chromosphäre zeigen in einem Bereich von $2^{\prime \prime}$ bis $10^{\prime \prime}$ zufällige Bewegungen, deren Ursache möglicherweise die Auslenkung der Flussröhren in subphotosphärischen, granularen Strömungen liegt. Unter 


\section{Ergebnisse}

Benutzung des Hot Cloud Modells erhalten von Uexküll et al. (1989) eine mittlere Geschwindigkeit chromosphärischer Strukturen von $4 \mathrm{~km} / \mathrm{s}$ und leiten hieraus kinetische Energiestromdichten von $F_{k i n}=0,8-4 \cdot 10^{3} \mathrm{erg} \mathrm{cm}^{-2} \mathrm{~s}^{-1}$ ab. Diese Energie ist bei weitem nicht hoch genug, um die Strahlungsverluste der Chromosphäre zu ersetzten. Für die magnetische Energiestromdichte verhält sich dieses jedoch anders, von Uexküll et al. (1989) berechnen Energiestromdichten des Magnetfeldes zwischen $F_{\text {mag }}=2,5 \cdot 10^{5} \mathrm{erg} \mathrm{cm}^{-2} \mathrm{~s}^{-1}$ und $F_{\text {mag }}=3,2 \cdot 10^{7} \mathrm{erg} \mathrm{cm}^{-2} \mathrm{~s}^{-1}$ und geben die für ihr Szenario maximal mögliche Energiestromdichte des Magnetfeldes mit $F_{\text {mag,max }}=10^{8} \mathrm{erg} \mathrm{cm}^{-2} \mathrm{~s}^{-1}$ an. Wellstein et al.(1998) untersuchen chromosphärische Geschwindigkeiten heller Netzwerkpunkte mit Maximalwerten von $v=16 \mathrm{kms}^{-1}$. Im Mittel finden sie $\bar{v}=4,1 \mathrm{~km} \mathrm{~s}^{-1}$ und erhalten einen Fluss von $F_{m a g}=4,4 \cdot 10^{5} \mathrm{erg} \mathrm{cm}^{-2} \mathrm{~s}^{-1}$. Bewegungen photosphärischer Strukturen wurden z.B. von Volkmer et al. (1995) untersucht. Sie finden für eine kleine magnetische Struktur quasi-periodische horizontale Bewegungen mit einer Amplitude von $v=2 \mathrm{kms}^{-1}$ und geben die Energiestromdichte vertikaler, magnetoakustischer Wellen mit $F_{\text {mag }}=1,6-2,3 \cdot 10^{7} \mathrm{erg} \mathrm{cm}^{-2} \mathrm{~s}^{-1}$ an.

Im Folgenden soll aus den vorliegenden hochaufgelösten Magnetogrammen eine grobe Abschätzung der Energiestromdichte $F_{\text {mag }}$ des Magnetfeldes aufgrund transversaler Anstöße gegeben werden.

\subsection{2 Änderung der Querschnittsfläche}

Als Grundlage dieser Abschätzung werden kurze Zeitserien von Magnetogrammen von 12,5 bis 33,3 Minuten Dauer benutzt. Die Magnetogramme werden mit der Linienschwerpunkts-Methode (COG), vgl. Kap. 4.4.4, erstellt und bezüglich der enthaltenen Intensitätsabhängigkeit korrigiert, siehe Kap. 4.4.5. Anhand der Flächenänderung der magnetischen Strukturen nach einem Zeitschritt soll die Geschwindigkeit ermittelt werden, mit der die umliegende Granulation das Magnetfeld deformiert. Eine exakte Korrelation der einzelnen Magnetfeldkarten ist Voraussetzung für die Bestimmung der Flächenänderung des Magnetfeldes; sie wird an den specklerekonstruierten Breitbandbildern vorgenommen und auf die Magnetfeldkarten übertragen.

Um eine Querschnittsänderung des Magnetfeldes detektieren zu können, muss für die Magnetfeldkarte wie auch in Kap. 5.1 ein Schwellwert definiert werden, so dass nur die Feldstärken oberhalb dieses Schwellwertes in einer Schwarzweißmaske erfasst werden. Die Masken der beiden aufeinanderfolgenden Magnetfeldkarten werden verglichen und hinzugekommene bzw. verschwundene Pixel jeder zusammenhängenden Struktur positiv bzw. negativ indiziert, so dass die Struktur durch die Indexzahl und die Richtung der Änderung durch das Vorzeichen eindeutig identifiziert ist. Abb. 5.27 demonstriert dieses am Beispiel des 6. Scans der Serie 11, hinzukommende Pixel sind hell, verschwindende Pixel sind dunkel dargestellt. Bei einem Rauschlevel von $\sigma=40$ Gauss wird der Schwellwert des Magnetfeldes für diese Auswertung auf den $3 \sigma$-Wert von 120 Gauss festgesetzt.

In den so präparierten Karten werden die Anzahlen der positiven und negativen Gebietsänderungen für jede Struktur ermittelt. Für die anschließende Geschwindigkeitsberechnung wird die Ge- 

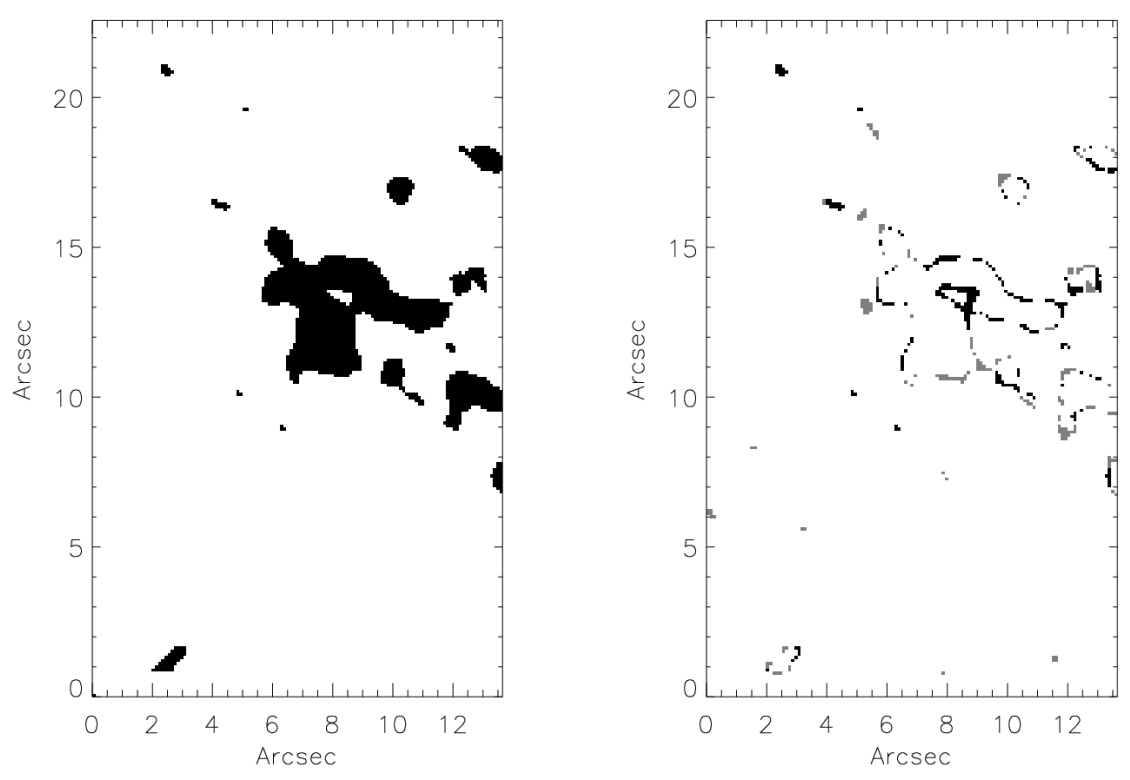

Abbildung 5.27: links: Magnetfeldkarte zu einem Zeitpunkt mit $B_{\|}>120$ Gauss, rechts: Indizierte Pixel der dazugekommenden (hell) und verschwindenden (dunkel) Gebiete.

samtänderung der Struktur, entsprechend der Summe der hinzukommenden und verschwindenden Pixel, zu Grunde gelegt.

\subsubsection{Horizontale Geschwindigkeiten}

Für die hier durchgeführte, einfache Abschätzung werden die magnetischen Strukturen "wider besseres Wissen", vgl. Kap. 5.1, in erster Näherung als kreisähnlich betrachtet, so dass für eine Änderung der Fläche $\Delta A$ in radialer Richtung um den Betrag $\Delta r$ gilt

$$
\Delta A=2 \pi r \cdot \Delta r .
$$

Diese Flächenänderung $\Delta A$ entspricht in den untersuchten Magnetogrammen der Anzahl $\Delta n$ der geänderten Pixel multipliziert mit dem Flächeninhalt $a=72,5^{2} \mathrm{~km}^{2}$ eines Pixels, also

$$
\Delta A=\Delta n \cdot a
$$

Die Gesamtfläche der beobachteten Struktur ergibt sich aus der Anzahl $n$ aller zu dieser Struktur gehörenden Pixel und der Pixelfläche $a$ zu $A=n \cdot a$ und entspricht der Fläche der theoretischen, kreisähnlichen Struktur von $A=\pi r^{2}$. Mit dieser Relation lässt sich der Radius $r$ der Struktur in 


\section{Ergebnisse}

Abhängigkeit von der Pixelanzahl $n$ ausdrücken durch

$$
r=\sqrt{\frac{n \cdot a}{\pi}}
$$

Einsetzen von Radius 5.9) und Flächenänderung 5.8 in Formel 5.7 liefert die Änderung des Radius in Abhängigkeit der Pixelanzahlen

$$
\Delta r=\sqrt{\frac{a}{4 \pi}} \cdot \frac{\Delta n}{\sqrt{n}}
$$

Aus dieser Radiusänderung kann nun mit der zeitlichen Differenz zweier aufeinanderfolgender Scans von $\Delta t=50 \mathrm{~s}$ die Geschwindigkeit der radialen Änderung zu $v=\frac{\Delta r}{\Delta t}$ abgeschätzt werden.

Für die in Abb. 5.27 gezeigte Serie 11 mit 15 Scans ergibt sich aus den 211 sich ändernden Strukturen von mehr als 120 Gauss eine mittlere Geschwindigkeit von

$$
\bar{v}=1,48 \mathrm{~km} \mathrm{~s}^{-1} \text {. }
$$

Die maximale Geschwindigkeit, die in dieser Serie gefunden wird, beträgt $v_{\max }=5,50 \mathrm{~km} \mathrm{~s}^{-1}$.

Serie 44, die mit 40 Scans einen längeren Zeitraum von 33,3 Minuten abdeckt und in Fe II bei $6149,2 \AA$ aufgezeichnet wurde, liefert ein ähnliches Ergebnis von $\bar{v}=1,68 \mathrm{~km} \mathrm{~s}^{-1}$, der Maximalwert dieser Serie liegt bei $v_{\max }=12,44 \mathrm{~km} \mathrm{~s}^{-1}$. Abb. 5.28 zeigt die Verteilung der 878 Geschwindigkeiten aus Serie 44.

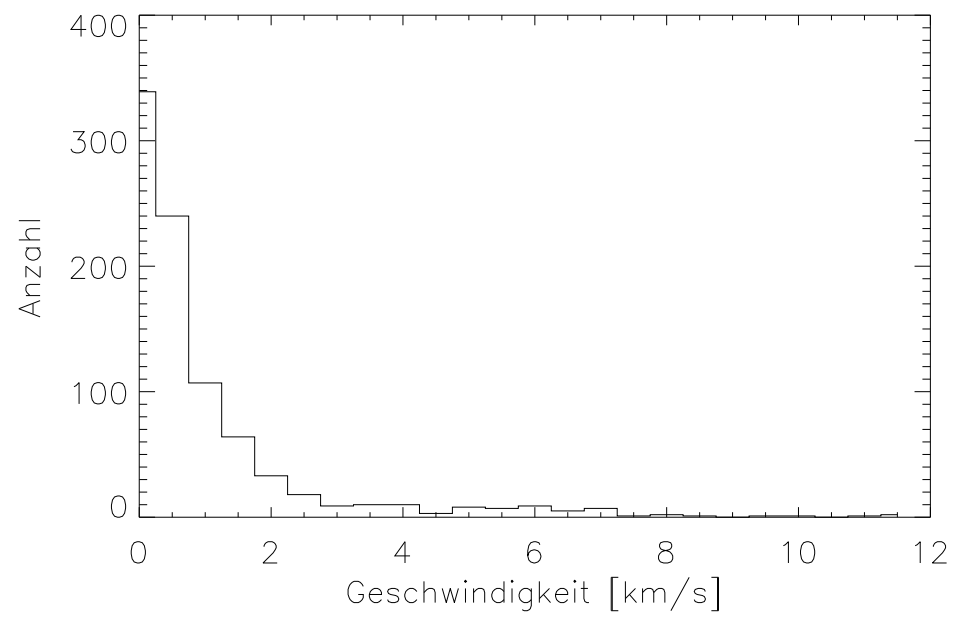

Abbildung 5.28: Mittlere Geschwindigkeit aus den 878 Strukturen der Serie 44 in Abhängigkeit vom Schwellwert.

Der Schwellwert des Magnetfeldes von 120 Gauss wirkt sich, wie Abb. 5.29 demonstriert, auf die Geschwindigkeitsbestimmung aus. Die starke Abhängigkeit für sehr geringe Schwellwerte wird hierbei dem durch Verarbeitung zweier Magnetogramme erhöhten Rauschen zugeschrieben. 
Die höheren Geschwindigkeiten für größere Schwellwerte könnten möglicherweise auf erhöhte Positionsänderungen der kompakten Flussröhren hindeuten, wobei die große Streuung der Geschwindigkeiten höherer Magnetfeldschwellwerte vermutlich durch die abnehmende Zahl der zu Grunde liegenden Strukturen bedingt ist.

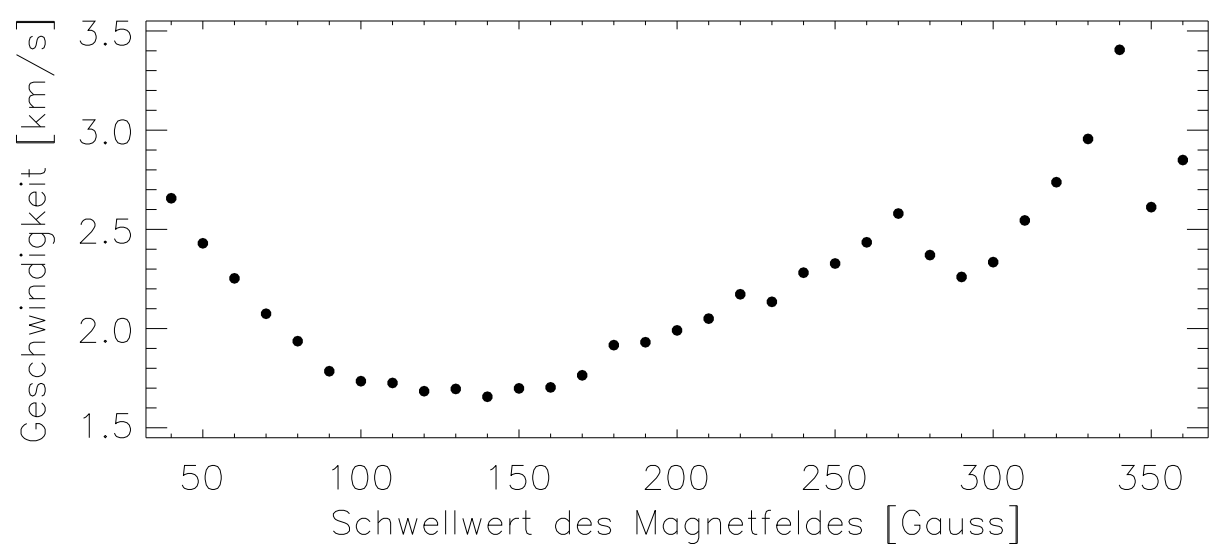

Abbildung 5.29: Verteilung der Geschwindigkeiten der 878 Strukturen von mehr als 120 Gauss in Serie 44.

Im Rahmen der Abschätzung wird wegen der Abhängigkeit der Geschwindigkeit vom Schwellwert des Magnetfeldes eine mittlere Geschwindigkeit der höheren Magnetfeldschwellwerte von $\bar{v}=2,5 \mathrm{~km} \mathrm{~s}^{-1}$ benutzt.

Auch die Abhängigkeit der berechneten Geschwindigkeiten vom Seeing soll untersucht werden. Dieser Punkt gewinnt an Bedeutung, da für die Geschwindigkeitsberechnung zwei aufeinanderfolgende Magnetfeldkarten subtrahiert werden, die unterschiedliche Seeing-Parameter aufweisen. Doch weder von der Qualität des Seeings, noch von der Differenz der zwei SeeingParameter wird eine Abhängigkeit festgestellt, vgl. Abb. 5.30. Dieses ist als Bestätigung der Bildrekonstruktion zu werten, die im idealen Fall die Einflüsse des Seeings komplett aus den Daten entfernt.

\subsubsection{Energiedichte}

Aus der Geschwindigkeit $v$ und der Änderung der Energiedichte $\Delta E$ des Magnetfeldes lässt sich nun die Energiezufuhr pro Zeit und Flächeneinheit, der Energiestrom $F$, berechnen durch

$$
F=\Delta E \cdot v .
$$

Hierbei kann die Energiedichteänderung $\Delta E$ des Magnetfeldes $B$ folgendermaßen abgeschätzt werden: 


\section{Ergebnisse}
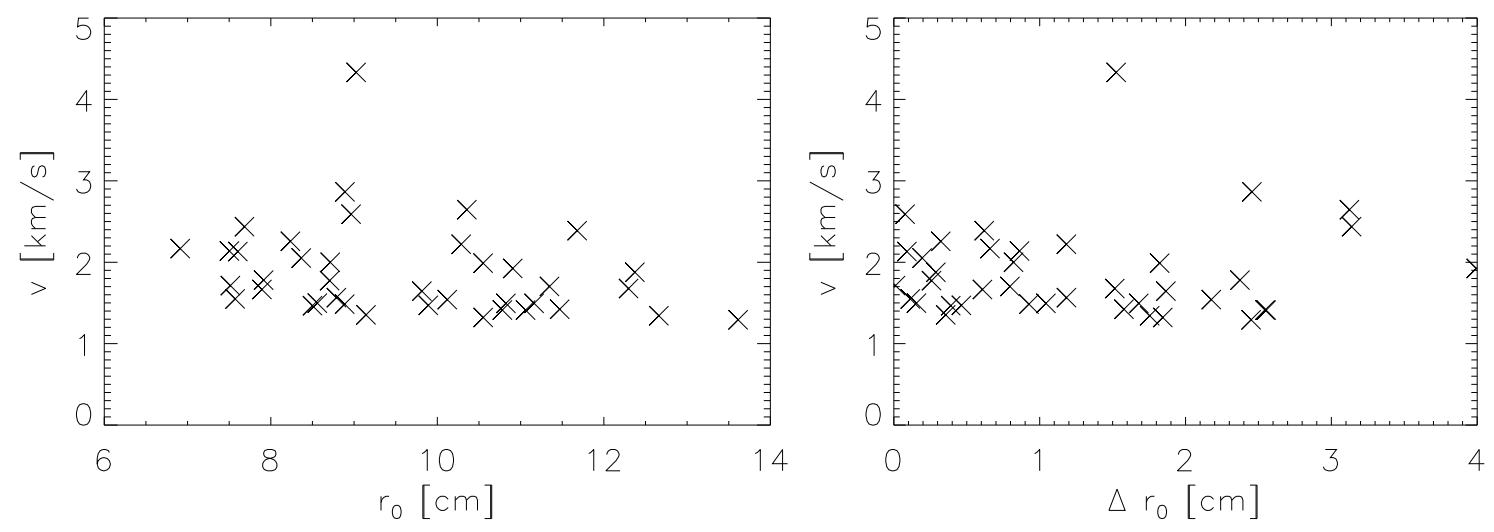

Abbildung 5.30: Geschwindigkeiten in Abhängigkeit vom Fried-Parameter (linkes Bild), bzw. von der Differenz der Fried-Parameter aufeinanderfolgender Magnetfeldkarten (rechtes Bild).

Die Energiedichte $E$ des Magnetfeldes am Ort der Messung ist im Gauss'schen Maßsystem gegeben durch

$$
E=\frac{B^{2}}{8 \pi},
$$

woraus sich die Änderung der Energiedichte zu $\Delta E=\frac{1}{4 \pi} B \cdot \Delta B$ ableiten lässt. Durch eine grobe Abschätzung der Magnetfeldänderung auf $\Delta B=0,1 B$ erhält man hieraus eine ungefähre Energiedichtenänderung von

$$
\Delta E=0,1 \cdot \frac{B^{2}}{4 \pi}
$$

Da in dem betrachteten Fall des solaren Magnetfeldes ein Auflösungselement nicht notwendig komplett mit Magnetfeld durchsetzt ist, muss für die Energiedichte $=\frac{\text { Energie }}{\text { Volumen }}$ anstelle des gemessenen Volumens des Auflösungselementes $V_{\text {mess }}$ das wahre Volumen des Magnetfeldes $V_{\text {wahr }}=\alpha V_{\text {mess }}$ betrachtet werden. Wird in Formel 5.13 das gemessene Volumen durch das wahre Volumen ersetzt, so ergibt sich für die Änderung der Energiediche eine Multiplikation mit dem Füllfaktor $\alpha$ :

$$
\Delta E=0,1 \cdot \frac{B^{2}}{4 \pi} \cdot \alpha
$$

Der Füllfaktor des Magnetfeldes kann aus den vorliegenden Daten nicht ermittelt werden. Um dennoch Eckwerte für den möglichen Energiestrom zu erhalten, werden exemplarisch zwei Extremfälle betrachtet:

1. Im einfachsten Fall wäre $\alpha=1$ und $B=120$ Gauss, so dass $B_{\text {eff }}=\alpha \cdot B=120$ Gauss. 
Hieraus ergibt sich die Energiedichteänderung zu

$$
\Delta E=0,1 \cdot \frac{B^{2}}{4 \pi}=115 \mathrm{erg} \mathrm{cm}^{-3}
$$

2. Es gilt als gesichert (Solanki|1993), dass die Feldstärken im chromosphärischen Netzwerk und in aktiven Gebieten im kGauss-Bereich liegen. Bei $B_{\text {eff }}=120$ Gauss schätzen wir somit $B \approx 1200$ Gauss und $\alpha \approx 0,1$ ab. Damit ist $B_{\text {eff }}=\alpha \cdot B=120$ Gauss und es folgt eine Energiedichteänderung von

$$
\Delta E=0,1 \cdot \frac{B^{2}}{4 \pi} \cdot 0,1=1150 \mathrm{erg} \mathrm{cm}^{-3}
$$

Nach den oben vorgenommenen Abschätzungen soll noch einmal betont werden, dass es sich bei der Magnetfeldstärke nur um die Sichtlinienkomponente des wahren Magnetfeldvektors handelt, so dass die Energiedichteänderung in diesem Punkt unterschätzt wird.

\subsubsection{Magnetischer Energiestrom}

Die Energiestromdichte, die Änderung der Energie pro Zeit und Flächeneinheit, kann nun aus den in Kap. 5.2 .3 berechneten Geschwindigkeiten von $\bar{v}_{\text {min }}=1,5 \cdot 10^{5} \mathrm{~cm} \mathrm{~s}^{-1}$ und $\bar{v}_{\text {max }}=2,5$. $10^{5} \mathrm{~cm} \mathrm{~s}^{-1}$ und den soeben abgeschätzten Energiedichten bestimmt werden zu

1. $F=1,5 \cdot 10^{7} \mathrm{erg} \mathrm{cm}^{-2} \mathrm{~s}^{-1}$ bei $B_{\|}=120$ Gauss, $\alpha=1$ und $v=1,5 \cdot 10^{5} \mathrm{~cm} \mathrm{~s}^{-1}$

2. $F=2,5 \cdot 10^{8} \mathrm{erg} \mathrm{cm}^{-2} \mathrm{~s}^{-1}$ bei $B_{\|}=1200$ Gauss, $\alpha=0,1$ und $v=2,5 \cdot 10^{5} \mathrm{~cm} \mathrm{~s}^{-1}$

Wegen der hohen Feldstärken in aktiven Gebieten (mit Füllfaktor 0,1) ist ein Wert von 1 $2 \cdot 10^{8} \mathrm{erg} \mathrm{cm}^{-2} \mathrm{~s}^{-1}$ (mit $v=1-2 \mathrm{~km} \mathrm{~s}^{-1}$ ) für den magnetischen Energiestrom plausibel. Die Größenordnung obiger Werte passt gut zu der Abschätzung des chromosphärischen und koronalen Energiebedarfs über aktiven Gebieten (Athay 1976; Withbroe 1976; Kopp \& Orrall 1976). Sie sind konsistent mit anderen Ergebnissen, z.B. von Volkmer et al. (1995). Der höhere Wert von $F=2,5 \cdot 10^{8} \mathrm{erg} \mathrm{cm}^{-2} \mathrm{~s}^{-1}$ liegt sogar nahezu eine Größenordnung über dem Energiebedarf.

Die Energiestromdichten werden hier nur aus den Änderungen der Ränder der Magnetfelder bestimmt. Es wird jedoch angenommen, dass innerhalb der magnetischen Strukturen ähnliche Energiestromdichten vorherrschen. Für eine genauere Abschätzung müsste daher die Feldverteilung und deren Änderung im Inneren der magnetischen Strukturen bekannt sein.

Es bleibt festzuhalten, dass die Änderung der Magnetfeldtopologie, gespeist aus der konvektiven Bewegung subphotosphärischer Schichten, für die Deckung des Energiebedarfs von Chromosphäre und Korona aufkommen kann. 


\subsection{Magnetfelder und Zero-Crossing-Geschwindigkeiten}

Aus dem Vorliegen kompletter Stokes- $V$-Profile für ein zweidimensionales Bildfeld ergeben sich weitere Optionen der Auswertung, auf die hier kurz eingegangen werden soll.

\subsubsection{Vergleich der Magnetfeldkarten}

Im Rahmen dieser Dissertation wurden neben den Magnetfeldkarten aus dem Linienschwerpunkt des Stokes- $I$-Signales auch Magnetfeldkarten aus der Amplitude des Stokes- $V$-Signales erstellt, siehe Kap. 4.4.3. Die Abb. 5.31 zeigt einen direkten Vergleich beider Methoden.
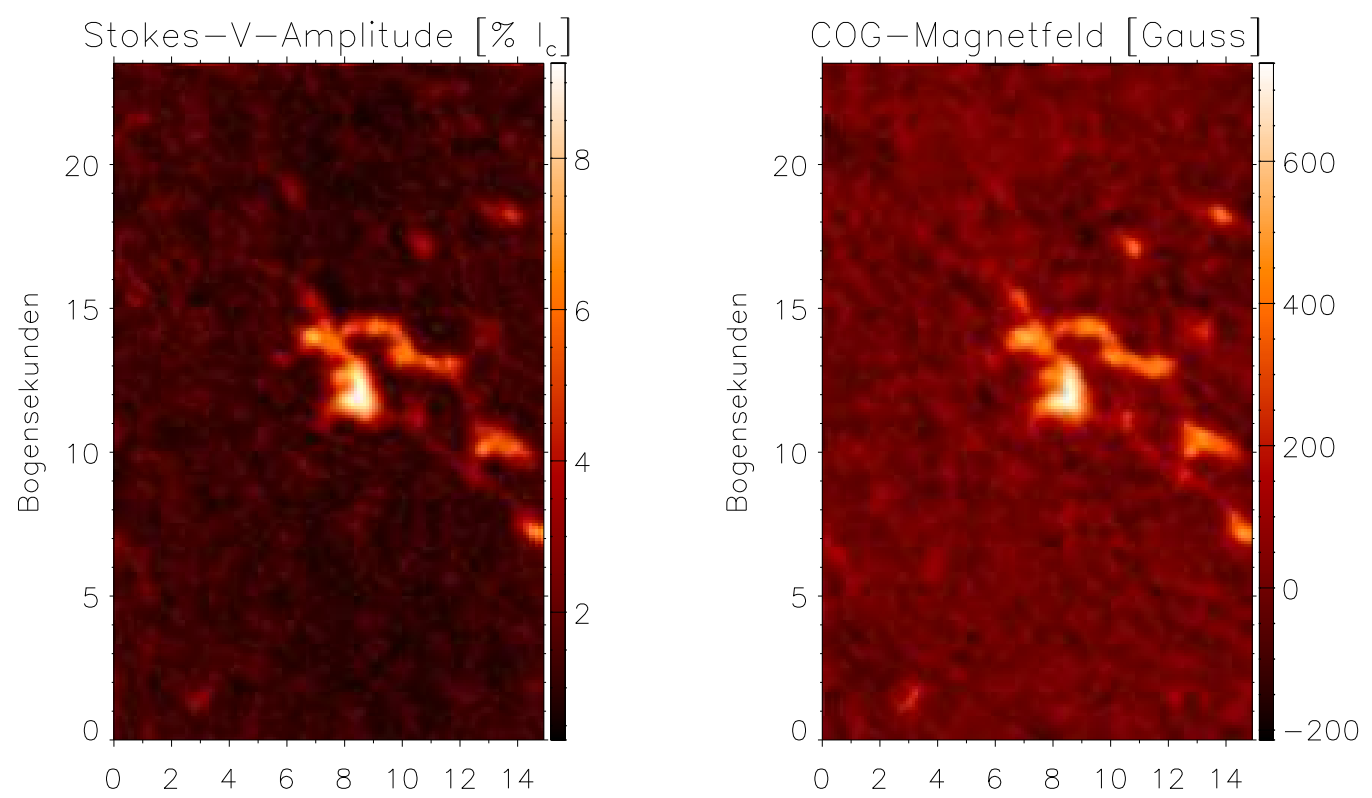

Abbildung 5.31: links: Magnetfeldkarte aus der Stokes- $V$-Amplitude in $\% I_{c}$, rechts: Magnetfeldkarte aus der Linienschwerpunktsmethode in Gauss.

Auf der linken Seite der Abb. 5.31 ist die Magnetfeldkarte der Stokes- $V$-Amplituden für Scan 11/6 dargestellt. Die Amplituden werden absolut gemessen, so dass die Polaritäten der Magnetfelder hier nicht unterschieden werden. Das stärkste Magnetfeld des gezeigten Scans besitzt eine Amplitude von gut $9 \%$ der Kontinuumsintensität. Die rechte Hälfte der Abbildung zeigt die Stärke des Magnetfeldes nach der Linienschwerpunktsmethode in Gauss. Hier sieht man, dass nur ein geringer Anteil an Magnetfeldern negativer Polarität in dem Bildfeld enthalten ist, der aber in der Darstellung eine Anhebung der Helligkeiten bewirkt. Die maximale, über ein Auflösungselement gemittelte Sichtlinienkomponente der Stärke des Magnetfeldes wird durch die COG-Methode zu 700 Gauss bestimmt. Beide Magnetfeldkarten stimmen, auch in den schwächer magnetischen Strukturen, sehr gut überein. Ihr Korrelationskoeffizient liegt bei 0,82, für Felder von mehr als 80 Gauss sogar bei 0,96. 
Noch deutlicher wird diese Übereinstimmung in der Abb. 5.32, einer direkten Auftragung der COG-Magnetfeldstärke gegen die Stokes- $V$-Amplitude aller Bildpunkte. Hier zeigt sich zwischen der Amplitude des Stokes- $V$-Signales und der COG-Magnetfeldstärke ein klarer linearer

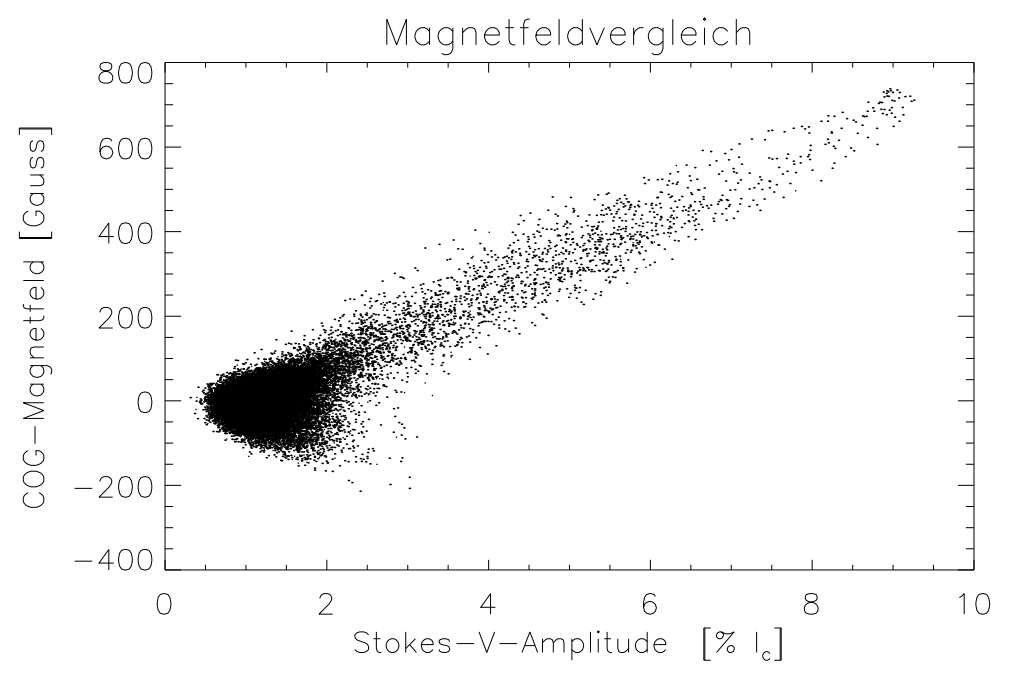

Abbildung 5.32: Vergleich der Magnetfeldstärke aus der Linienschwerpunktsmethode mit den Stokes- $V$-Amplituden für alle Ortspunkte des Scans 11/6.

Zusammenhang, der darauf hindeutet, dass die Weak-Field-Approximation für den untersuchten Scan erfüllt ist. Da die Amplituden von Stokes- $V$ im Betrag betrachtet werden, erscheint der kurze Ast negativer Polaritäten rückläufig.

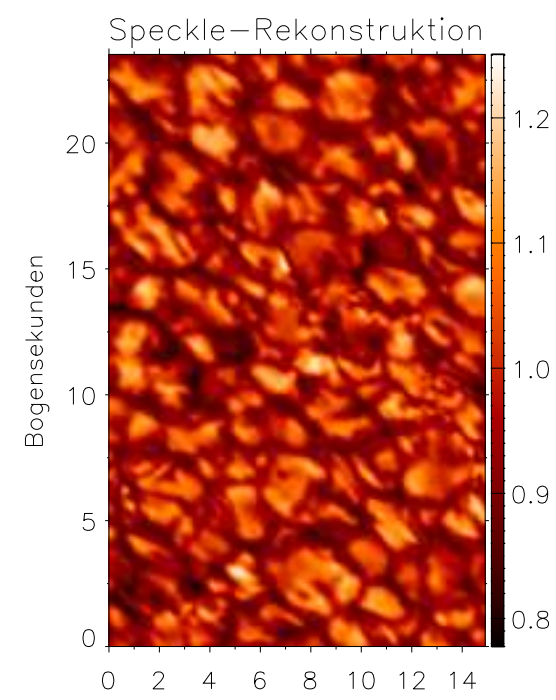

Abbildung 5.33: Specklerekonstruiertes Breitbandbild des Scans 11/6, auf $I_{c}$ normiert. 


\section{Ergebnisse}

Abb. 5.33 beinhaltet zur Veranschaulichung das specklerekonstruierte Breitbandbild des Scans 11/6, dessen Magnetfelder verglichen wurden, ein Plage-Gebiet bei $\cos \theta=0,82$.

\subsubsection{Zero-Crossing Geschwindigkeit}

Für die in Kap. 5.1 und 5.2 dargestellten Ergebnisse wurden wegen der höheren Genauigkeit dieser Methode ausschliesslich die COG-Magnetfelder benutzt. Doch die Stokes- $V$-Profile besitzen über die Berechnung von Magnetfeldkarten aus der Amplitude hinaus einen eigenen Wert, da in ihnen weitere Informationen über die magnetischen Strukturen der Sonne enthalten sind. So können über die Verschiebung des Nulldurchgangs (engl. zero crossing) des $V$-Profiles die vertikale Geschwindigkeit des magnetischen Anteils der Strukturen und über die Asymmetrie eines Stokes- $V$-Profils vertikale Geschwindigkeiten untersucht werden (Solanki 1993). Auf eine dieser Optionen, die Bestimmung der Geschwindigkeit magnetischer Strukturen durch den Stokes- $V$-Nulldurchgang, soll hier kurz eingegangen werden.

Für die Untersuchung der Geschwindigkeiten magnetischer Strukturen bergen die Stokes- $V$ Profile ein großes Potential: Die von der Polarisation abhängige Absorption, die zu dem beobachteten Stokes- $V$-Profil führt, findet ausschließlich in den magnetischen Bereichen statt, daher enthält das Stokes- $V$-Profil ausschließlich Informationen aus magnetischen Gebieten. Die Geschwindigkeiten, die aus dem Schwerpunkt von Stokes-I gewonnen werden, enthalten dahingegen immer Informationen über beide, magnetische und unmagnetische Anteile eines Auflösungselementes. Anhand der aus dem Stokes- $V$-Signal gewonnenen Geschwindigkeit können so die Strömungen der magnetischen Elemente gezielt untersucht werden, auch wenn diese räumlich nicht aufgelöst werden.

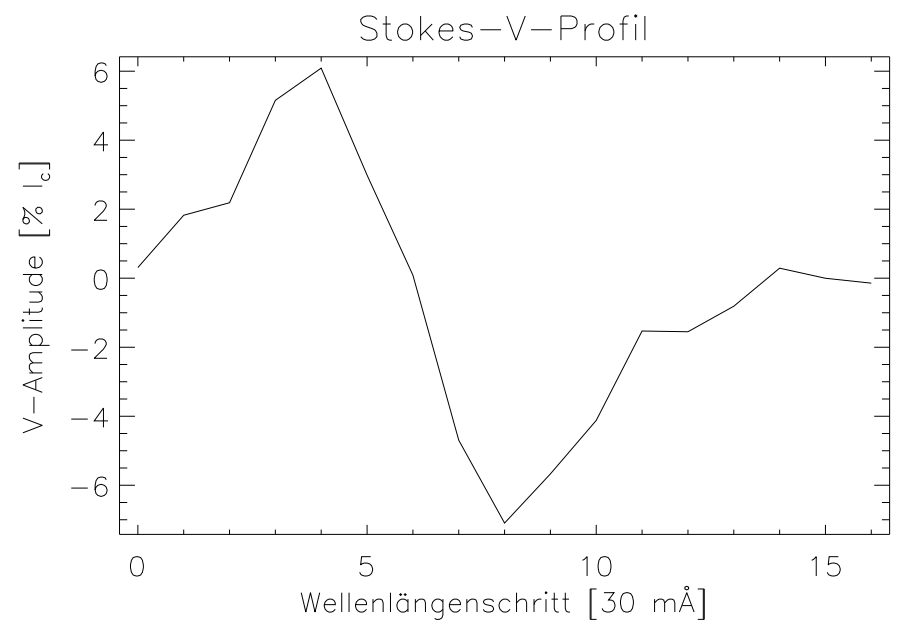

Abbildung 5.34: Stokes- $V$-Profil mit durchschnittlicher Amplitude von $6 \% I_{c}$.

Für die Bestimmung dieser Geschwindigkeit wird der innere Teil des Stokes- $V$-Profiles, s. Abb. 5.34, durch ein Polynom dritten Grades gefittet und dessen Nulldurchgang wird bestimmt. Die 


\subsection{Magnetfelder und Zero-Crossing-Geschwindigkeiten}

Dopplerverschiebung des Nulldurchgangs lässt auf die Geschwindigkeit schliessen, vgl. Kap. 4.5.2. Da für diese Geschwindigkeitsbestimmung keine Referenzwellenlänge zur Verfügung steht, wird als Nullpunkt die mittlere Stokes-I-Geschwindigkeit benutzt.

Stokes- $V$-Profile stehen für jeden Bildpunkt des zweidimensionalen Bildfeldes zur Verfügung, so dass auf diese Weise Geschwindigkeitskarten der magnetischen Anteile der Pixel erstellt werden können. Abb. 5.35 zeigt die Geschwindigkeitskarten aus Stokes- $I$ und Stokes- $V$ im Vergleich.
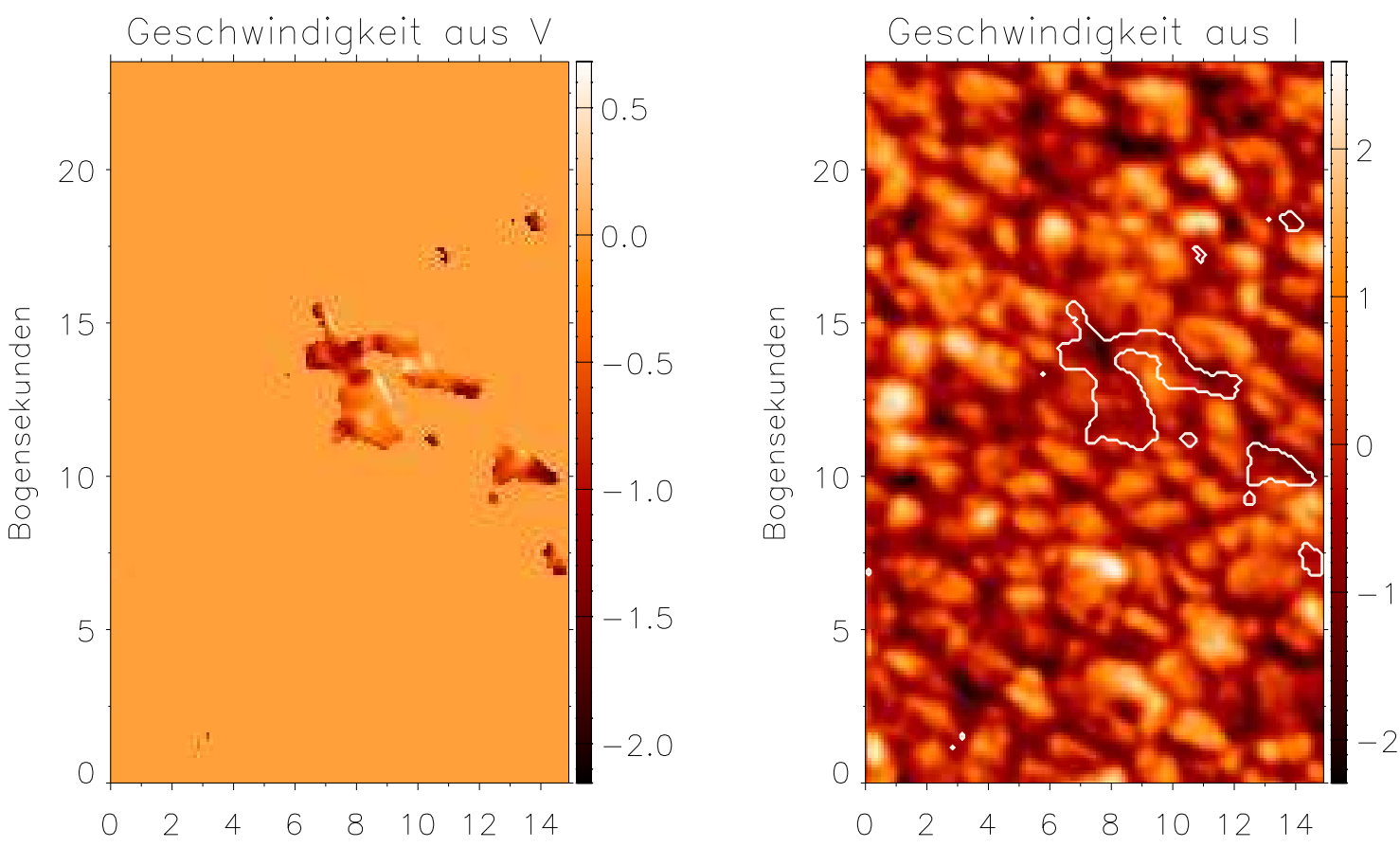

Abbildung 5.35: links: Geschwindigkeiten magnetischer Strukturen aus Stokes- $V$ in $\mathrm{km} \mathrm{s}^{-1}$, rechts: Geschwindigkeiten aller Strukturen aus Stokes- $I$ in $\mathrm{km} \mathrm{s}^{-1}$.

Obwohl in der Stokes- $V$-Geschwindigkeitskarte, Abb. 5.35 links, auch geringe positive Geschwindigkeiten auftreten, dominieren die negativen Geschwindigkeiten mit Abströmungen bis $\mathrm{zu} 2 \mathrm{~km} \mathrm{~s}^{-1}$, wie auch von Solanki (1993) erwartet. Die rechte Abbildung zeigt, dass die mittleren Geschwindigkeiten der magnetischen und unmagnetischen Anteile wie erwartet gut mit der Intensität korreliert sind (vgl. Abb. 5.33). Zur Orientierung wurde die Konturlinie des Magnetfeldes in diese Abbildung übernommen. In den magnetischen Gebieten werden mäßige mittlere Geschwindigkeiten detektiert.

Eine weitere Option für die Auswertung ist die Untersuchung von Wellen innerhalb magnetischer Strukturen, die durch die Zerocrossinggeschwindigkeiten gefunden werden könnten. Ein Beispiel solcher Wellen wurde bereits von Volkmer et al. (1995) vorgestellt. Eine detailliertere Untersuchung dieser Fragestellung ist sicher von Interesse. Sie ist mit dem vorhandenen Datenmaterial möglich und soll in einer späteren Arbeit durchgeführt werden. Möglicherweise wird sie 


\section{Ergebnisse}

Aufschluss über magnetische Wellenphänomene (magneto-akustische Wellen) und deren Einfluss auf die Atmosphärenstruktur geben. 


\subsection{Morphologie und Dynamik: "Bright Points" und strukturierte Gebiete}

In diesem Abschnitt sollen exemplarisch morphologische Charakterisierungen und zeitliche Variationen dargestellt werden. Aus der Vielzahl der beobachteten Magnetfeldstrukturen und Zeitabläufe werden einige typische herausgegriffen. Es sei vorab angemerkt, dass keine Systematik gefunden werden konnte in dem Sinne, dass sich kausale Abfolgen von Konvektion und magnetischer Strukturbildung angeben lassen. Vielmehr findet man auf kleinen Skalen im Bereich von $1^{\prime \prime}$ und darunter eher ein chaotisches, zufallsbedingtes Verhalten. Es lässt sich aber festhalten, dass sich die Magnetfelder sehr dynamisch verhalten, vgl. auch Koschinsky (2001); Koschinsky et al. (2001).

Alle der folgenden Abbildungen sind nach dem gleichen Prinzip aufgebaut. Sie bestehen aus drei Spalten von Teilbildern, wobei die linke Spalte jeweils den Ausschnitt des specklerekonstruierten Breitbandbildes normiert auf die Kontinuumsintensität zeigt, die mittlere Spalte gibt die Magnetfeldstärken der Linienschwerpunktsmethode in Gauss wieder, und die rechte Spalte beinhaltet die aus dem Linienschwerpunkt von Stokes- $I$ bestimmten Geschwindigkeiten in $\mathrm{km} \mathrm{s}^{-1}$. Die räumliche Größe der Bilder ist durch Markierungen am Bildrand in Bogensekunden angegeben. Jede Spalte besteht ihrerseits aus fünf Teilbildern, die sich durch eine zeitliche Distanz unterscheiden. Sofern nichts anderweitiges angegeben ist, beträgt der Zeitabstand der Bilder $100 \mathrm{~s}$ und die Bildgröße $5^{\prime \prime} \times 5^{\prime \prime}$. Die in den Abbildungen erkennbaren Quadrate stellen die Pixelgröße der Kamera dar.

Die erste Abbildung, Nr. 5.36, zeigt ein Magnetfeld mit einer Stärke von 800 Gauss in Scheibenmitte, das stark auf kleinstrukturierten Gebieten konzentriert ist. Das Magnetfeld liegt verschoben zu den kleinskaligen Aufhellungen, es ist mit der anomalen Granulation verknüpft, d.h. mit modifizierter Konvektion.

Im Gegensatz zum ersten Beispiel ist in der Specklerekonstruktion der Abb. 5.37(Serie 11) eine starke, kleinskalige Aufhellung (Fackelpunkt) begleitet von einem ebenfalls sehr kleinskaligen, deutlichen Magnetfeld von 300 Gauss zu sehen. Während der dargestellten 8 Minuten bewegt sich die helle Struktur um etwa eine Bogensekunde von rechts unten nach links oben, wobei die gleiche Bewegung auch in der Magnetfeldkarte zu verzeichnen ist. Das Bildfeld dieser Teilbilder besitzt abweichend zu den vorigen eine Größe von $4^{\prime \prime} \times 4^{\prime \prime}$.

Abb. 5.38 zeigt einen mit $7^{\prime \prime} \times 7^{\prime \prime}$ etwas größeren Ausschnitt der gleichen Serie. Der zeitliche Abstand der Teilbilder wurde hier zu $\Delta t=150$ s gewählt, so dass eine Gesamtdauer von 12 Minuten überblickt werden kann. In diesem Zeitraum werden mehrere starke Aufhellungen im Breitbandbild sichtbar, der Zusammenhang zwischen Magnetfeld und starker Intensität scheint fast zufälliger Natur zu sein, es ist auch hier eine räumliche Übereinstimmung von Magnetfeld und anomaler Granulation gegeben.

Während eines Intervalls von 8 Minuten verschwindet in Abb. 5.39 ein Fackelpunkt im linken oberen Quadranten, während in der Mitte des Bildfeldes einer neuer entsteht. Das Magnetfeld entwickelt sich hiervon unbehelligt; es teilt sich in drei Einzelgebiete auf und verbindet sich 


\section{Ergebnisse}

wieder. Nur in den letzten Zeitschritten ist ein deutlicher Zusammenhang zu erkennen. Die Abbildung zeigt $5^{\prime \prime} \times 5^{\prime \prime}$ große Ausschnitte bei $\cos \theta=0,56$.

Abb. 5.40 ist im Gegensatz zu allen vorherigen Beispielen dieses Kapitels nicht in der Eisenlinie bei $6302,5 \AA$, sondern bei $6149,2 \AA$ entstanden. Sie zeigt Ausschnitte von $7^{\prime \prime} \times 7^{\prime \prime}$ nahe der Scheibenmitte bei $\cos \theta=0,92$, in denen ein stark strukturiertes Magnetfeld von bis zu 800 Gauss zu sehen ist. Selbst in der kurzen Zeitdifferenz zweier Bilder von $\Delta t=50 \mathrm{~s}$ ist eine Umstrukturierung des Magnetfeldes zu erkennen, einhergehend mit starken Änderungen des Intensitäts- und Strömungsmusters. Es werden Abwärtsströmungen von bis zu $2 \mathrm{~km} / \mathrm{s}$ gemessen.

Die ebenfalls in Fe II bei 6149,2 $\mathrm{A}$ aufgezeichnete Abb. 5.41 zeigt im linken oberen Quadranten der specklerekonstruierten Breitbandbilder eine in ihrem Zentrum kollabierende Struktur, zu erkennen an dem zugehörigen Strömungsring. Die Beobachtungszeit dieser Struktur beträgt 4 Minuten, bereits nach 3 Minuten ist die Struktur komplett zerfallen. Die Dynamik des sich stark ändernden Magnetfeldes ist jedoch durch andere Konvektionsvorgänge in der rechten Hälfte des Bildfeldes bestimmt.

Während einer Dauer von 12 Minuten zeigt die Abb. 5.42 der gleichen Serie einen gerade entstandenen Fackelpunkt, der sich innerhalb von knapp 3 Minuten auf zwei einzelne Strukturen aufteilt. Nach weiteren 3 Minuten sind beide Strukturen wieder verbunden und gehen im weiteren Verlauf in die Unscheinbarkeit über. Auf das sich stetig ändernde Magnetfeld hat dieser Vorgang jedoch wenig Einfluss. Die Stokes-I-Geschwindigkeiten dieses über 33 Minuten relativ ortskonstanten Magnetfeldes zeigen sehr wenig Strömung an.

Zusammenfassend lässt sich folgern, dass die beobachteten Magnetfelder eine hohe Dynamik zeigen, ein klarer Zusammenhang zur Intensität der Strukturen ist jedoch nicht feststellbar. 

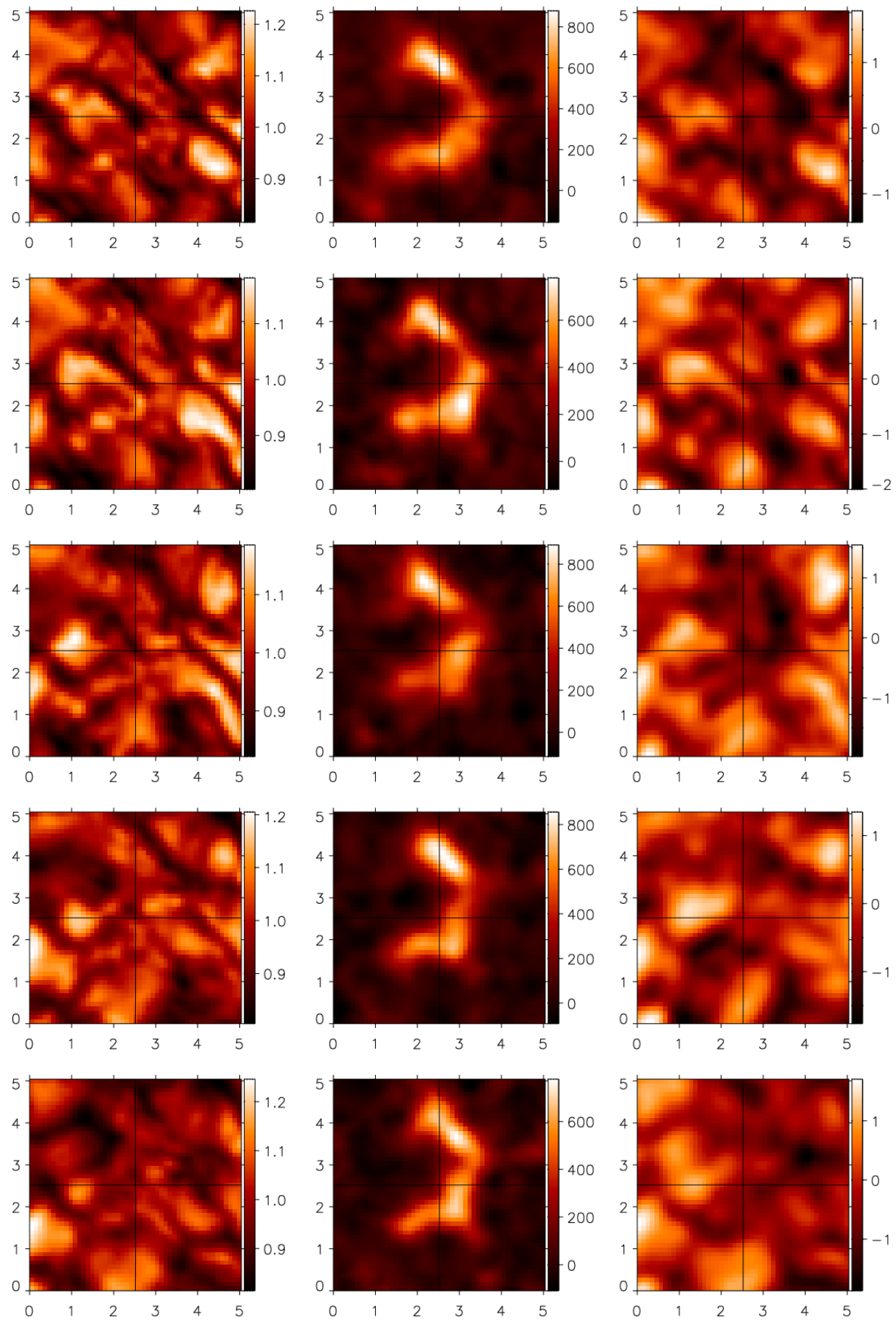

Abbildung 5.36: Zeitliche Änderung von oben nach unten mit $\Delta t=100 \mathrm{~s}$, Bildfeld $5^{\prime \prime} \times 5^{\prime \prime}$ Serie 10 bei $\cos \theta=0,99$; alle Skalen relativ zum Ausschnittsmittelwert links: Specklerekonstruiertes Breitbandbild auf $\bar{I}$ normiert mitte: Magnetfeld der Linienschwerpunktsmethode in Gauss rechts: Stokes- $I$-Geschwindigkeiten in $\mathrm{km} \mathrm{s}^{-1}$, hell aufwärts 


\section{Ergebnisse}
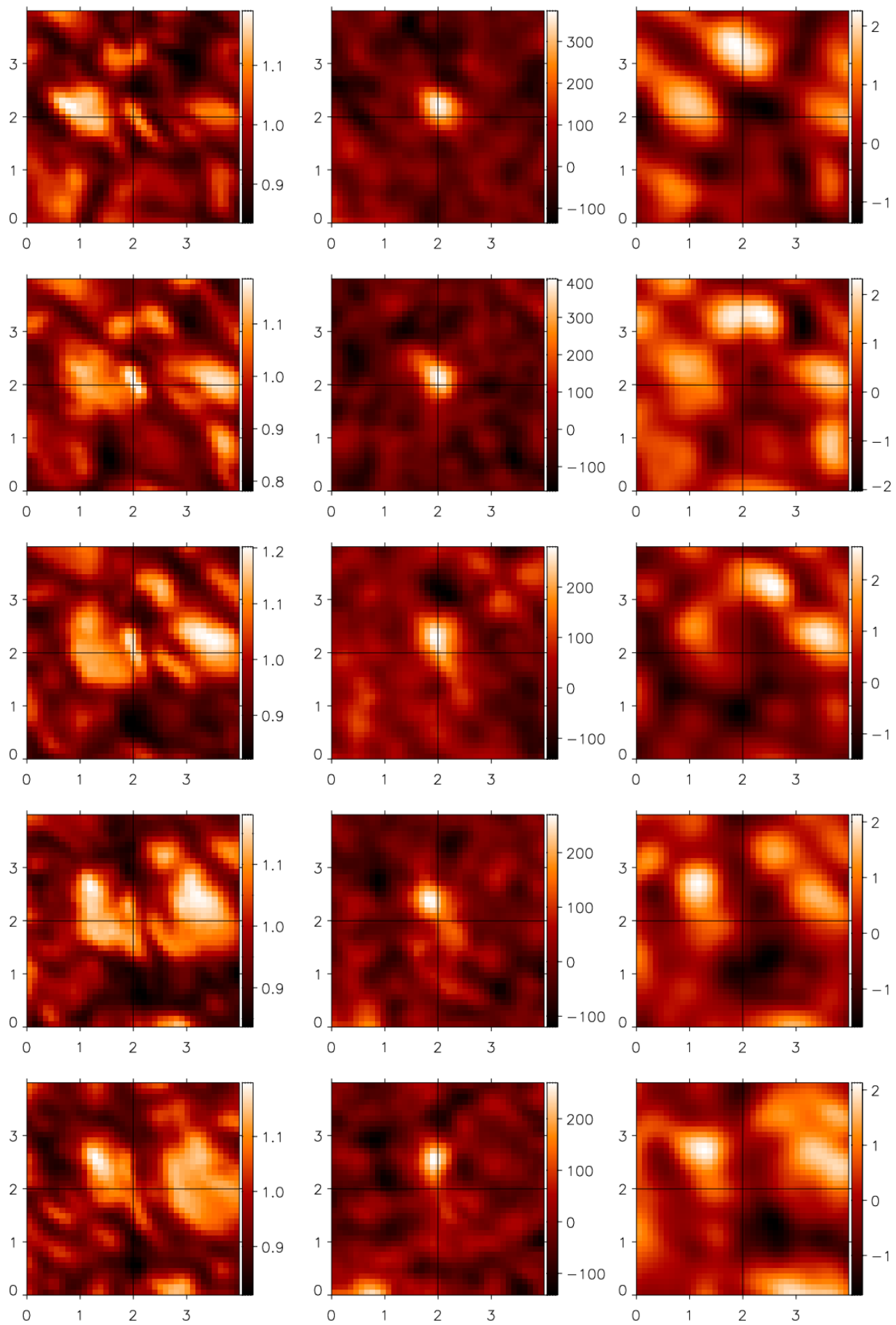

Abbildung 5.37: Zeitliche Änderung von oben nach unten mit $\Delta t=100 \mathrm{~s}$, Bildfeld $4^{\prime \prime} \times 4^{\prime \prime}$ Serie 11 bei $\cos \theta=0,82$; alle Skalen relativ zum Ausschnittsmittelwert links: Specklerekonstruiertes Breitbandbild auf $\bar{I}$ normiert mitte: Magnetfeld der Linienschwerpunktsmethode in Gauss rechts: Stokes- $I$-Geschwindigkeiten in $\mathrm{km} \mathrm{s}^{-1}$, hell aufwärts 

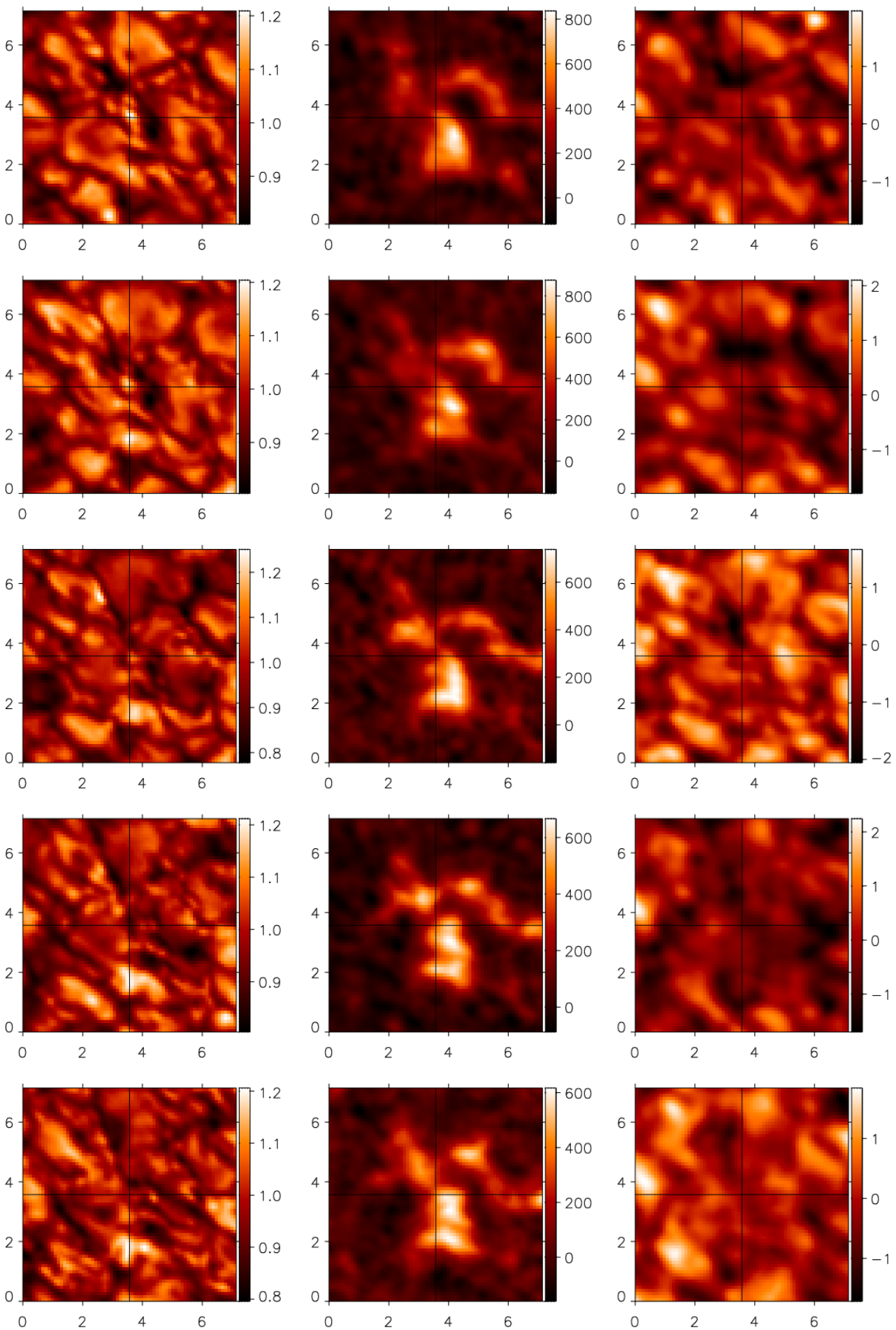

Abbildung 5.38: Zeitliche Änderung von oben nach unten mit $\Delta t=150 \mathrm{~s}$, Bildfeld $7^{\prime \prime} \times 7^{\prime \prime}$ Serie 11 bei $\cos \theta=0,82$; alle Skalen relativ zum Ausschnittsmittelwert links: Specklerekonstruiertes Breitbandbild auf $\bar{I}$ normiert mitte: Magnetfeld der Linienschwerpunktsmethode in Gauss rechts: Stokes- $I$-Geschwindigkeiten in $\mathrm{km} \mathrm{s}^{-1}$, hell aufwärts 


\section{Ergebnisse}
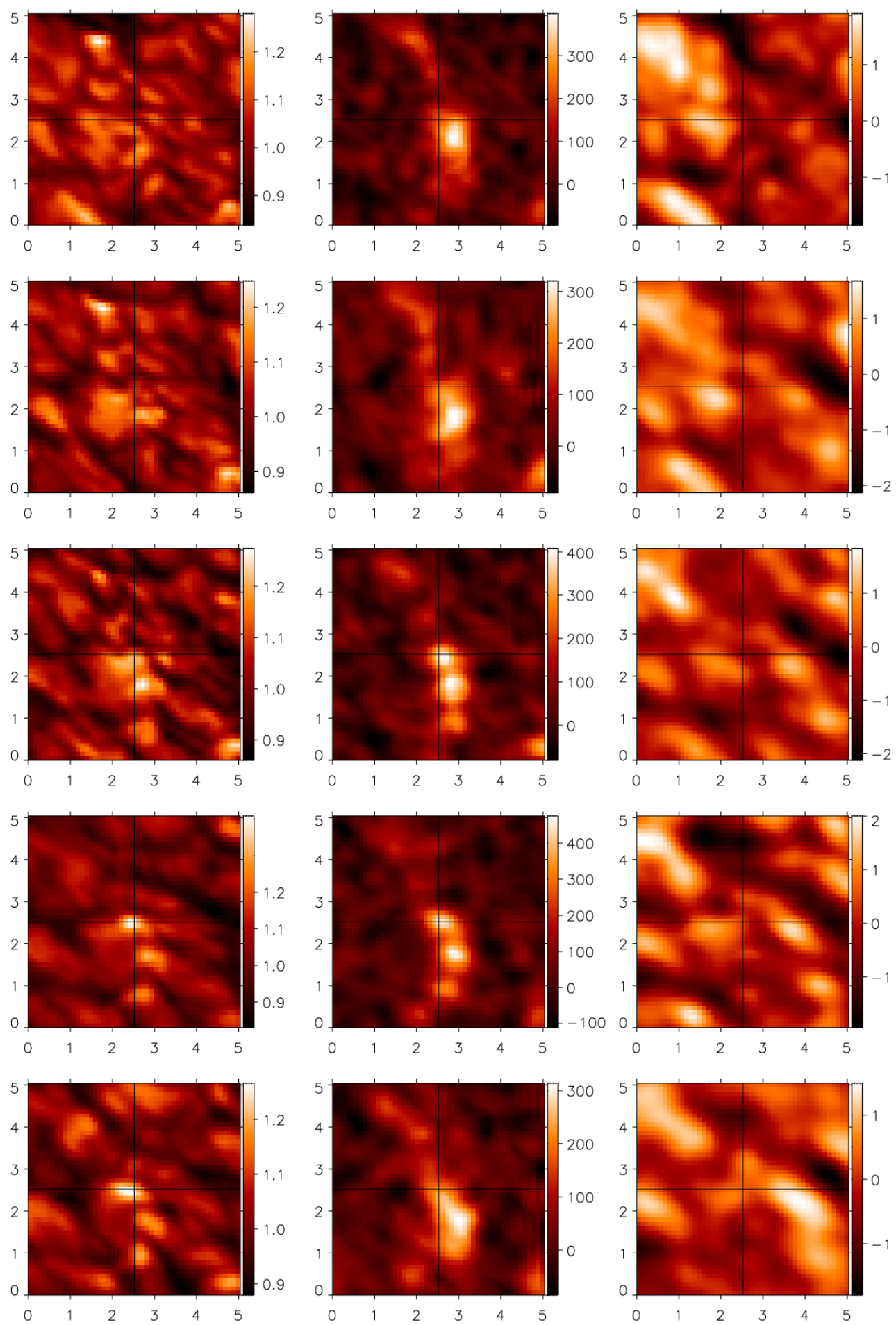

Abbildung 5.39: Zeitliche Änderung von oben nach unten mit $\Delta t=100 \mathrm{~s}$, Bildfeld $5^{\prime \prime} \times 5^{\prime \prime}$ Serie 13 bei $\cos \theta=0,56$; alle Skalen relativ zum Ausschnittsmittelwert links: Specklerekonstruiertes Breitbandbild auf $\bar{I}$ normiert mitte: Magnetfeld der Linienschwerpunktsmethode in Gauss rechts: Stokes- $I$-Geschwindigkeiten in $\mathrm{km} \mathrm{s}^{-1}$, hell aufwärts 

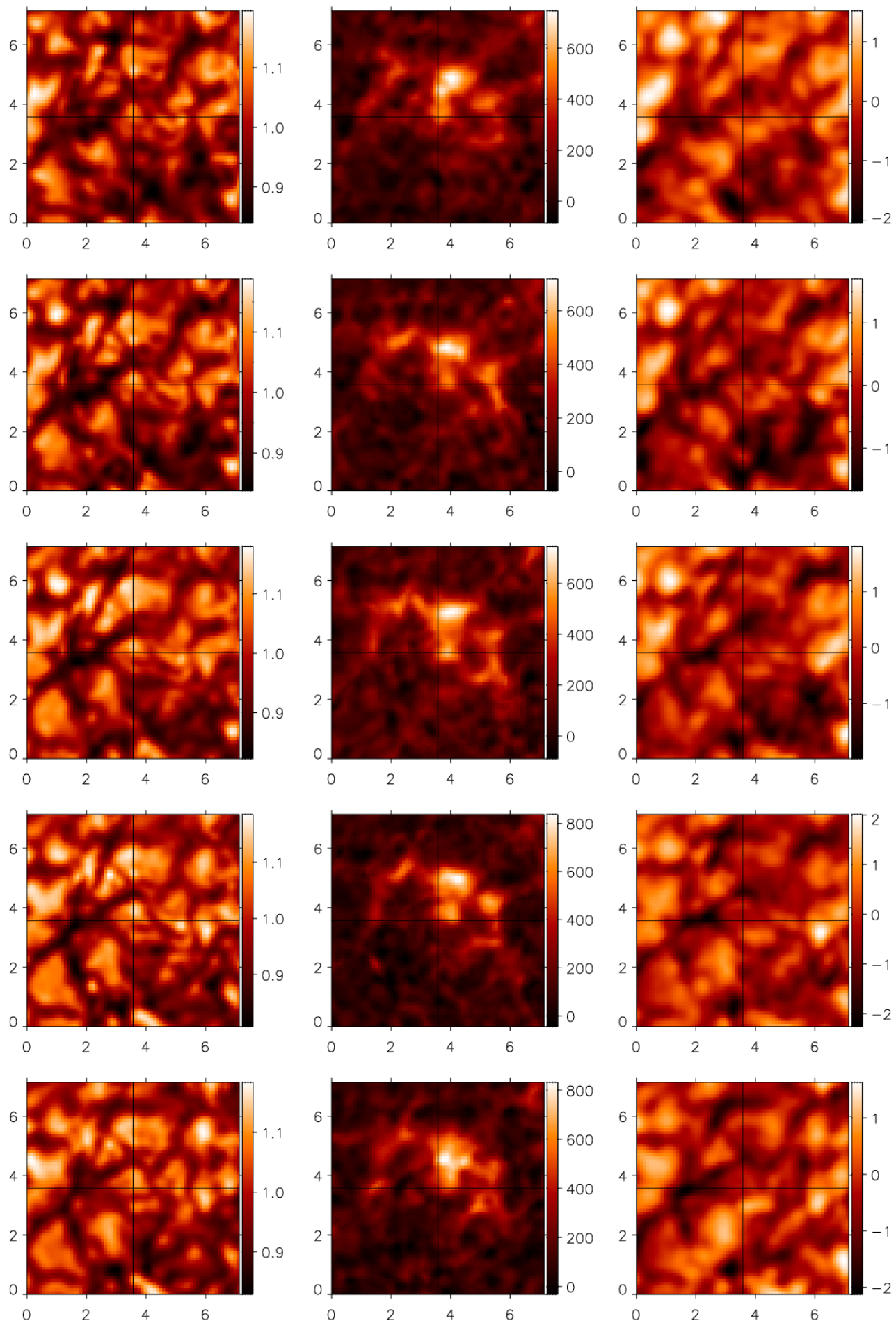

Abbildung 5.40: Zeitliche Änderung von oben nach unten mit $\Delta t=50 \mathrm{~s}$, Bildfeld $7^{\prime \prime} \times 7^{\prime \prime}$ Serie 37 bei $\cos \theta=0,92$; alle Skalen relativ zum Ausschnittsmittelwert links: Specklerekonstruiertes Breitbandbild auf $\bar{I}$ normiert mitte: Magnetfeld der Linienschwerpunktsmethode in Gauss rechts: Stokes- $I$-Geschwindigkeiten in $\mathrm{km} \mathrm{s}^{-1}$, hell aufwärts 


\section{Ergebnisse}
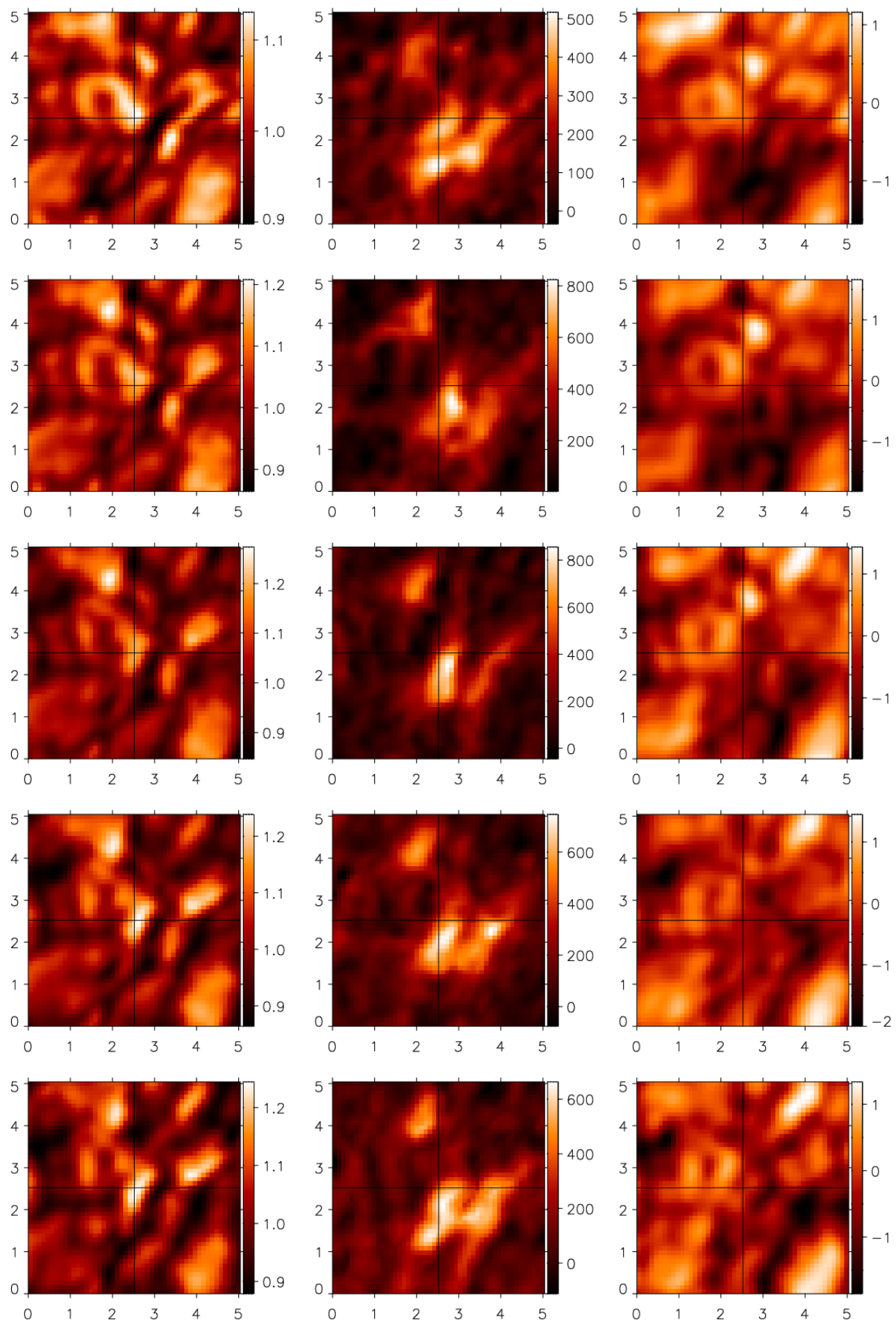

Abbildung 5.41: Zeitliche Änderung von oben nach unten mit $\Delta t=50 \mathrm{~s}$, Bildfeld $5^{\prime \prime} \times 5^{\prime \prime}$ Serie 44 bei $\cos \theta=0,80$; alle Skalen relativ zum Ausschnittsmittelwert links: Specklerekonstruiertes Breitbandbild auf $\bar{I}$ normiert mitte: Magnetfeld der Linienschwerpunktsmethode in Gauss rechts: Stokes- $I$-Geschwindigkeiten in $\mathrm{km} \mathrm{s}^{-1}$, hell aufwärts 

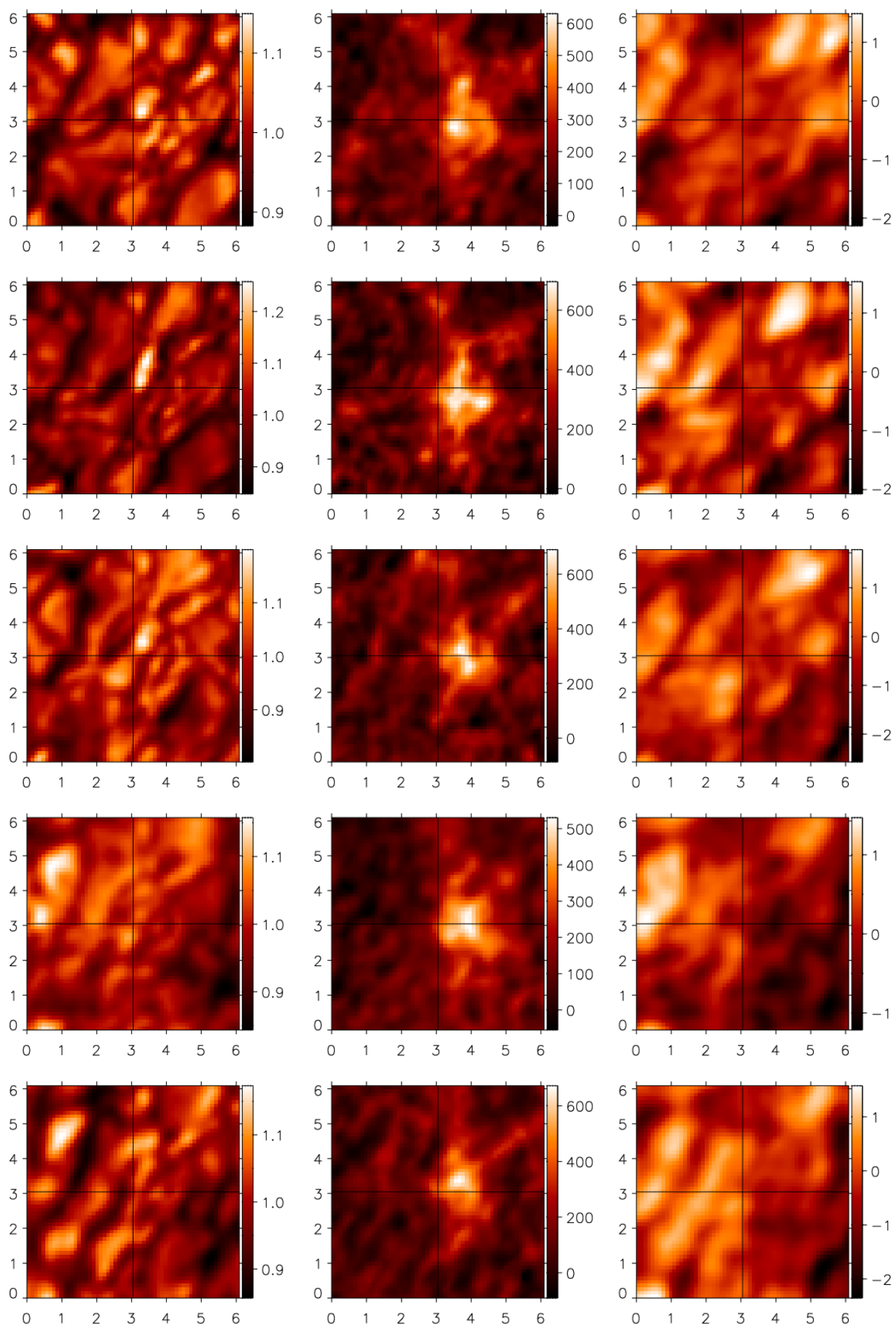

Abbildung 5.42: Zeitliche Änderung von oben nach unten mit $\Delta t=150 \mathrm{~s}$, Bildfeld $6^{\prime \prime} \times 6^{\prime \prime}$ Serie 44 bei $\cos \theta=0,80$; alle Skalen relativ zum Ausschnittsmittelwert links: Specklerekonstruiertes Breitbandbild auf $\bar{I}$ normiert mitte: Magnetfeld der Linienschwerpunktsmethode in Gauss rechts: Stokes- $I$-Geschwindigkeiten in $\mathrm{km} \mathrm{s}^{-1}$, hell aufwärts 
5 Ergebnisse 


\section{Zusammenfassung und Ausblick}

Zur Zeit werden weltweit große Anstrengungen unternommen, die "magnetische" Sonne zu verstehen, insbesondere auf kleinen Skalen. So war es Ziel dieser Dissertation, das Verhalten und die Struktur kleinskaliger Magnetfelder in der Photosphäre der Sonne zu untersuchen.

Um dieses zu bewerkstelligen, wurden in acht Beobachtungskampagnen am Vakuum Turm Teleskop, Teneriffa, an verschiedenen solaren Positionen polarimetrische Daten in den Linien Fe I 6302,5 $\AA$ und Fe II 6149,2 $\AA$ aufgezeichnet. Mit dem Göttinger Spektropolarimeter konnten Scans von jeweils 147 schmalbandigen Spektrogrammen in 21 Wellenlängenpositionen simultan mit 147 Breitbandbildern aufgezeichnet werden. Durch Anwendung der Methode der SpeckleRekonstruktion konnte die Qualität der Breitbandbilder entscheidend verbessert werden, so dass die Auflösungsgrenze der specklerekonstruierten Breitbandbilder bei etwa $0,2^{\prime \prime}-0,3^{\prime \prime}$ liegt. Die 147 Bilder eines schmalbandigen Wellenlängenscans wurden zu einem hochaufgelösten Schmalbandscan rekonstruiert. Der dazu benutzte Programmcode wurde für die vorliegende Arbeit grundlegend überarbeitet. Nach der Rekonstruktion der Schmalbanddaten wurden die vollen Stokes- $V$-Profile durch Subtraktion und die Stärke des Magnetfeldes mit der Linienschwerpunktsmethode aus den zwei polarimetrischen Kanälen bestimmt. Als Nebenprodukt konnte die Geschwindigkeit aus der Dopplerverschiebung des Schwerpunktes von Stokes-I mitberechnet werden. Auch aus der Amplitude des Stokes- $V$-Signales wurde eine Magnetfeldkarte erstellt und aus dem Nulldurchgang des $V$-Profiles die Geschwindigkeit der magnetischen Anteile eines Auflösungselementes bestimmt.

\section{Hausdorff-Dimension magnetischer Strukturen}

Um die Selbstähnlichkeit magnetischer Strukturen zu untersuchen, wird unter Benutzung der COG-Magnetfelder die Hausdorff-Dimension des Randes magnetischer Strukturen über mehrere Größenskalen berechnet. Für einen Schwellwert der Magnetfeldstärke von 80 Gauss ergibt sich unter Benutzung des korrekten $G$-Maßstabes eine fraktale Dimension von $D=1,39 \pm 0,05$. Die Selbstähnlichkeit magnetischer Strukturen, die von Stenflo \& Holzreuter (2003a) postuliert wurde, ist für einen festen Schwellwert gegeben. Nur die größten gefundenen Strukturen von mehr als 316 Pixeln, für die in dieser Untersuchung keine ausreichende Statistik vorliegt, scheinen eine größere Dimension des Randes zu besitzen. Unabhängig hiervon ist jedoch festzustellen, dass die Hausdorff-Dimension des Randes auch von dem Schwellwert der magnetischen Strukturen abhängt. Je höher hierbei die Feldstärke desto weniger komplex ist der Rand. Die fraktalen Dimensionen fallen hierbei von $D_{b}=1,39$ für 80 Gauss auf $D_{b}=1,22$ für 320 Gauss ab. Dieses 


\section{Zusammenfassung und Ausblick}

Verhalten ist konsistent mit den Ergebnissen von Meunier (1999), die für kleinere Strukturen ebenfalls einen Abfall der Dimension mit höheren Magnetfeldschwellwerten findet.

Der Vergleich der fraktalen Dimensionen beobachteter und numerisch simulierter Daten verläuft sehr gut. Die MHD-Simulationen mit einem anfänglichen, homogenen Magnetfeld von 50 Gauss, siehe Vögler \& Schüssler (2003), liefern zunächst höhere Dimensionen als die Beobachtungen, durch Anpassen des Verhältnisses von $G$-Maßstab zur Auflösung der betrachteten Daten relativiert sich der Unterschied zu $\Delta D=0,01$ bzw. 0,06, abhängig von der Methode der Anpassung (s. Kap. 5.1.8 und 5.1.9). Aus der guten Übereinstimmung lässt sich eine große Realitätsnähe der Simulationen folgern, die einen solchen Vergleich möglich macht und die Möglichkeit aufzeigt, aus den Simulationen über die Struktur und Dynamik der solaren Magnetfelder zu lernen.

\section{Energiezufuhr durch Umstrukturierung des Magnetfeldes}

Der Versuch einer groben Abschätzung der durch Anstöße der umgebenden Granulation an eine magnetische Struktur abgegebene Energie zeigt, dass durch diese Prozesse genügend Energie zur Verfügung gestellt wird und sie mitverantwortlich sein könnten für die Heizung der höheren Schichten der Sonnenatmosphäre.

Für diese Untersuchung werden die horizontalen Geschwindigkeiten, die an den Rändern des Magnetfeldes auftreten, aus der Flächenänderung des Magnetfeldes berechnet und mit einer abgeschätzten Energiedichte des Magnetfeldes in eine Energiestromdichte umgesetzt. Die auftretenden Geschwindigkeiten ergeben sich hierbei zu 1,5-2,5 $\mathrm{km} \mathrm{s}^{-1}$. Mit der Abschätzung der Änderung der Magnetfeldstärke um $10 \%$ ergibt sich für Magnetfelder von $B_{\text {eff }}=120$ Gauss die Änderung der Energiedichten zu $\Delta E=115-1150 \mathrm{erg} \mathrm{cm}^{-3}$, abhängig von der wahren Magnetfeldstärke $B$ und dem Füllfaktor $\alpha$. Die gesamte Energiestromdichte der magnetischen Strukturen ergab sich damit zu Werten von $F=1,5 \cdot 10^{7} \mathrm{bis} 2,5 \cdot 10^{8} \mathrm{erg} \mathrm{cm}^{-2} \mathrm{~s}^{-1}$. Diese Werte passen gut zu dem Energiebedarf der Chromosphäre und Korona über aktiven Gebieten (Athay 1976).

\section{Magnetfelder und Zero-Crossing Geschwindigkeiten}

Durch die Stokes- $V$-Profile ergeben sich weitere Optionen der Auswertung. So wurden aus den Amplituden des Stokes- $V$-Signales qualitative Magnetfeldkarten erstellt. Diese Karten reichen in der Aussagekraft nicht an die der Linienschwerpunktsmethode heran, doch der Vergleich beider Methoden festigt das Vertrauen in die Ergebnisse: Die Magnetfeldkarten aus Stokes- $V$ Amplituden und der COG-Methode stimmen sehr gut überein.

Aus dem Nulldurchgang der Stokes- $V$-Profile kann wie auch aus dem Schwerpunkt des StokesI-Profiles über den Doppler-Effekt eine Geschwindigkeit berechnet werden. Die Geschwindigkeitskarten aus dem Stokes- $V$-Nulldurchgang besitzten hierbei den entscheidenden Vorteil, dass sie ausschliesslich die Geschwindigkeiten zeigen, die in den magnetischen Anteilen der Auflösungselemente vorherrschen, während die Stokes-I-Geschwindigkeiten das Mittel aller im Auflösungselement enthaltenen Strukturen darstellen. 
Die Stokes- $V$-Geschwindigkeiten wurden exemplarisch für einen Scan berechnet, wobei sich durchweg abwärtsgerichtete Strömungen in den magnetischen Anteilen der Auflösungselemente zeigen. Das Interesse gilt hierbei nicht nur den Stokes- $V$-Geschwindigkeiten selbst, sondern auch der Möglichkeit, vertikale Wellenphänomene der Magnetfelder aufzuspüren. Für einen Einzelfall ist Volkmer et al. (1995) dieses bereits gelungen, eine weitergehende Untersuchung dieser Optionen wird folgen.

\section{Morphologie und Dynamik}

Anhand der vorliegenden, hochaufgelösten, zweidimensionalen Bildfelder ist es möglich, die Struktur und Entwicklung der photosphärischen Magnetfelder im Detail zu untersuchen. Eine Auswahl interessanter Ausschnitte wurde hierzu in Kap. 5.4 zusammengestellt.

Zusammenfassend kann festgestellt werden, dass magnetische Strukturen eine große Dynamik aufweisen. Sie ändern ihre Gestalt innerhalb weniger Minuten und darunter. Starke Magnetfelder werden teilweise bei kleinskaligen hellen Strukturen (Fackelpunkten) gefunden, der Großteil der beobachteten Magnetfelder ist jedoch mit sehr kleinstrukturierten Gebieten anomaler Granulation und somit mit modifizierter Konvektion verbunden. Ein klarer Zusammenhang zwischen Magnetfeld und Intensität konnte nicht festgestellt werden.

\section{Ausblick}

Das vorliegende, ausgewertete Datenmaterial erlaubt wie oben angedeutet weitere Untersuchungen. So kann die magnetische Energiezufuhr durch topologische Änderungen genauer als oben durchgeführt abgeschätzt werden, indem geometrische Strukturänderungen für verschiedene effektive Feldstärken durchgeführt werden. Die zeitlichen Verläufe der Geschwindigkeiten aus den Stokes- $V$-Profilen erlauben, systematische Strömungen innerhalb kleinskaliger Magnetfelder und magnetische Wellenphänomene zu studieren. Die Untersuchung der beobachtbaren Wechselwirkung der granularen Konvektion mit dem Magnetfeld steht erst am Anfang und Vergleiche mit numerischen Simulationen werden mehr Klarheit in das zunächst unverstandene Verhalten der beobachteten magnetischen Strukturen und ihrer granularen Umgebung schaffen.

Schließlich sind weitere Durchbrüche mit verbesserten Beobachtungsmöglichkeiten zu erwarten, die da wären die Entwicklungen in der Detektortechnologie, in Adaptiver Optik zur "online"Korrektur des Seeings und die neuen Sonnenteleskope mit großer Öffnung wie das deutsche 1,5 m GREGOR-Teleskop, welches gerade gebaut wird, und das mit internationaler Beteiligung geplante amerikanische $4 \mathrm{~m}$ ATST. 
6 Zusammenfassung und Ausblick 


\section{Literaturverzeichnis}

Adjabshirizadeh A., Koutchmy S., 2002, Center-limb and shape-factor variations of facular elements, in A. Wilson (ed.), Solar Variability: From Core to Outer Frontiers, Vol. 506, ESA, p. 415

Athay R., 1976, The solar chromosphere and corona: Quiet Sun, No. 53 in Astrophysics and space science library, D. Reidel

Auffret H., Muller R., 1991, Center-to-limb variation of the network bright points in the solar photosphere, A\&A 246, 264

Balke A. C., Schrijver C. J., Zwaan C., Tarbell T. D., 1993, Percolation theory and the geometry of photospheric magnetic flux concentrations, Solar Phys. 143, 215

Beckers J. M., 1969, A table of Zeeman Multiplets, Sacramento Peak Observatory Contribution no.141 - Physical Science Research Papers no. 371, Sacramento: Peak Observatory and Air Force Cambridge Research Laboratories, 1969

Bendlin C., 1993, Hochauflösende zweidimensionale Spektroskopie der solaren Granulation mit einem Fabry-Perot-Interferometer, Dissertation, Georg-August-Universität, Göttingen

Berger T. E., Title A. M., 1996, On the Dynamics of Small-Scale Solar Magnetic Elements, ApJ 463, 365

Bovelet B., Wiehr E., 2001, A new algorithm for pattern recognition and its application to granulation and limb faculae, Solar Phys. 201, 13

Brault J., White O., 1971, The Analysis and Restoration of Astronomical Data via the Fast Fourier Transform, A\&A 13, 169

Capitani C., Landi degl'Innocenti E., Cavallini F., Ceppatelli G., Landi degl'Innocenti M., 1989, Polarization properties of a 'Zeiss-type' coelostat - The case of the solar Tower in Arcetri, Solar Phys. 120, 173

Cauzzi G., Smaldone L. A., Balasubramaniam K. S., Keil S. L., 1993, On the calibration of line-of-sight magnetograms, Solar Phys. 146, 207 
Literaturverzeichnis

de Boer C. R., 1993, Speckle-Interferometrie und ihre Anwendungen auf die Sonnenbeobachtung, Dissertation, Universität Göttingen

de Boer C. R., Kneer F., 1992, Speckle observations of abnormal solar granulation, A\&A 264, L24

Deinzer W., Hensler G., Schüssler M., Weisshaar E., 1984, Model Calculations of Magnetic Flux Tubes - Part Two - Stationary Results for Solar Magnetic Elements, A\&A 139, 435

Falconer K., 1990, Fractal Geometry - Mathematical Foundations and Applications, John Wiley \& Sons

Foukal P., 1993, The Curious Case of the Greenwich Faculae, Solar Phys. 148, 219

Greimel R., Brandt P. N., Guenther E., Mattig W., 1990, On the fractal dimension of the solar granulation, Vistas in Astronomy 33, 413

Haken, Wolf, 1993, Atom- und Quantenphysik, Springer, Berlin

Hale G. E., 1908, The Zeeman Effect in the Sun, PASP 20, 287

Hirzberger J., 1998, Analyse von Zeitserien räumlich hochaufgelöster Aufnahmen der Sonnengranulation, Dissertation, Karl-Franzens-Universität, Graz

Hirzberger J., Vazquez M., Bonet J. A., Hanslmeier A., Sobotka M., 1997, Time Series of Solar Granulation Images. I. Differences between Small and Large Granules in Quiet Regions, ApJ 480, 406

Howard R. F., 1996, Solar active regions as diagnostics of subsurface conditions, A\&A 34, 75

Keller C. U., von der Luehe O., 1992, Solar speckle polarimetry, A\&A 261, 321

Knox K. T., Thompson B. J., 1974, Recovery of images from atmospherically degraded shortexposure photographs, ApJ 193, L45

Kopp R., Orrall F., 1976, Energy Balance and Structure of Active Regions, in R.-M. Bonnet, P. Delache (eds.), The energy balance and hydrodynamics of the solar chromosphere and corona, No. 36 in I.A.U. Colloquium, 457

Korff D., 1973, Analysis of a Method for Obtaining Near Diffraction Limited Information in the Presence of Atmospheric Turbulence, Optical Society of America Journal 63, 971

Koschinsky M., 2001, Spektroskopie und Polarimetrie kleinskaliger magnetischer Strukturen der Sonnenoberfläche mit Methoden der Bildrekonstruktion, Dissertation, Georg-AugustUniversität, Göttingen

Koschinsky M., Kneer F., Hirzberger J., 2001, Speckle spectro-polarimetry of solar magnetic structures, A\&A 365, 588 
Labeyrie A., 1970, Attainment of Diffraction Limited Resolution in Large Telescopes by Fourier Analysing Speckle Patterns in Star Images, A\&A 6, 85

Landi Degl'Innocenti E., 1992, Magnetic field measurements, in Solar Observations: Techniques and Interpretation, p. 71

Lovejoy S., 1982, Area-perimeter relation for rain and cloud areas, Science 216, 185

Mandelbrot B. B., 1997, The Fractral Geometry of Nature, Chapt. 12, 109 ff, W.H. Freeman and Company, New York

Martínez Pillet V., Lites B. W., Skumanich A., 1997, Active region magnetic fields. I. Plage fields, ApJ 474, 810

Meunier N., 1999, Fractal Analysis of Michelson Doppler Imager Magnetograms: A Contribution to the Study of the Formation of Solar Active Regions, ApJ 515, 801

Moore C. E., 1945, A multiplet table of astrophysical interest, Washington

Moore C. E., 1966, The Solar Spectrum 2935 A to 8770 A, Vol. Monograph 61, National Bureau of Standards, Washington

Narain U., Ulmschneider P., 1996, Chromospheric and Coronal Heating Mechanisms II, Space Science Reviews 75, 453

Roudier T., Muller R., 1987, Structure of the solar granulation, Solar Phys. 107, 11

Rüedi I., Solanki S. K., Livingston W. C., 1995, Infrared lines as probes of solar magnetic features. X. HeI 10830A as a diagnostic of chromospheric magnetic fields., A\&A 293, 252

Sánchez Almeida J., Martínez Pillet V., 1994, The Inclination of Network Magnetic Fields, ApJ 424, 1014

Sanchez Almeida J., Martinez Pillet V., Wittmann A. D., 1991, The instrumental polarization of a Gregory-coude telescope, Solar Phys. 134, 1

Schatten K. H., Mayr H. G., Omidvar K., Maier E., 1986, A hillock and cloud model for faculae, ApJ 311, 460

Schrijver C. J., Zwaan C., Balke A. C., Tarbell T. D., Lawrence J. K., 1992, Patterns in the photospheric magnetic field and percolation theory, A\&A 253, L1

Schüssler M., 2003, MHD simulations: what's next?, in J. Trujillo Bueno, Sánchez Almeida, J. (eds.), Third International Workshop on Solar Polarization, im Druck

Semel M., 1967, Contribution à létude des champs magnétiques dans les régions actives solaires, Annales d'Astrophysique 30, 513 
Literaturverzeichnis

Socas-Navarro H., 1999, NLTE Inversion of spectral lines and stokes profiles, Dissertation, La Laguna

Solanki S. K., 1993, Small-scale solar magnetic fields: an overview, Space Sci. Rev. 63, 1

Spruit H. C., 1976, Pressure equilibrium and energy balance of small photospheric fluxtubes, Solar Phys. 50, 269

Stellmacher G., Wiehr E., 2001, Spatial offsets between lines and continuum in limb faculae, Solar Phys. 202, 259

Stenflo J., Holzreuter R., 2003a, Distribution of magnetic fields at scales beyond the spatial resolution limit, ASP Conf. Ser., im Druck

Stenflo J., Holzreuter R., 2003b, Empirical view of magnetoconvection, ESA Publ., im Druck SP-505

Stenflo J., Holzreuter R., 2003c, Flux tubes or fractal distributions - on the nature of photospheric magnetic fields, Astron. Nachr., im Druck

Stix M., 1989, The Sun, A\&A Library, Berlin Springer

Sütterlin P., 1996, Über die Struktur von solaren Poren, Dissertation, Universität Freiburg

Sütterlin P., Wiehr E., Stellmacher G., 1999, Continuum photometry of solar white-light faculae, Solar Phys. 189, 57

Tarbell T., Ferguson S., Frank Z., Shine R., Title A., Topka K., Scharmer G., 1990, HighResolution Observations of Emerging Magnetic Fields and Flux Tubes in Active Region Photosphere, in IAU Symposium, p. 147

Vögler A., 2003, Dissertation, Georg-August-Universität, Göttingen

Vögler A., Schüssler M., 2003, Studying magneto-convection by numerical simulation, Astron. Nachr. 324(4), 399

Voigt H. H., 1991, Abriss der Astronomie, BI-Wiss., Mannheim, 5. Auflage

Volkmer R., 1995, Hochauflösende zweidimensionale Spektro-Polarimetrie solarer Magnetfelder, Dissertation, Georg-August-Universität, Göttingen

Volkmer R., Kneer F., Bendlin C., 1995, Short-period waves in small-scale magnetic flux tubes on the sun, A\&A 304

von der Lühe O., 1984, Estimating Fried's parameter from a time series of an arbitrary resolved object imaged through atmospheric turbulence, Optical Society of America Journal 1, 510

von Uexküll M., Kneer F., Malherbe J. M., Mein P., 1989, Oscillations of the sun's chromosphere. V-Importance of network dynamics for chromospheric heating, A\&A 208, 290 
Weigelt G. P., 1977, Modified astronomical speckle interferometry 'speckle masking', Optics Communications 21, 55

Wellstein S., Kneer F., von Uexkuell M., 1998, Oscillations of the Sun's chromosphere, VIII Horizontal motions of Ca II K bright points, A\&A 335, 323

Withbroe G., 1976, Mass and Energy Flow in the Solar Atmosphere - Implications of Skylab Observations, in R.-M. Bonnet, P. Delache (eds.), The energy balance and hydrodynamics of the solar chromosphere and corona, No. 36 in I.A.U. Colloquium, 263 



\section{Danksagung}

Mein größter Dank gilt meinem Doktorvater, Franz Kneer, der mir während der gesamten Zeit der Dissertation an der Sternwarte ständig mit Rat und Tat zur Seite stand und immer für eine Diskussion Zeit hatte.

Für viele Gespräche und eine gute Atmosphäre in der Sternwarte möchte ich der gesamten Sonnenphysikgruppe danken. Namentlich erwähnt seien hier Markus Koschinsky, der in der Anfangsphase meiner Dissertation eine grosse Stütze war, Maren Wunnenberg, die mich das gesamte Studium mit vielen Gesprächen, wissenschaftlicher und persönlicher Natur, begleitete und Markus Sailer, der mir besonders in der Endphase seelischen Beistand gab.

Ausserhalb der Göttinger Gruppe gilt mein besonderer Dank Pit Sütterlin, der viele Anregungen und Hilfen bei der Softwareentwicklung gab und auch zu nachtschlafenden Zeiten noch zu Diskussionen bereit war.

Bernd Beyerstedt, Sven Blank, Tabea Sprenger und Anja vom Stein danke ich für das Korrekturlesen der Dissertation.

Für die Finanzierung dieser Dissertation sei der Deutschen Forschungsgemeinschaft gedankt, die die Arbeit als Träger des Graduiertenkollegs "Strömungsinstabilitäten und Turbulenz" und mit dem Projekt KN 152/25-1 unterstützte.

Bei Harald Gottschalk möchte ich mich für die Nachsicht in angespannten Situtationen und die Bekräftigung in meinem Tun bedanken. Für den Beistand im täglichen Leben danke ich Aneta Kiebala und Tania Garfias Marcedo.

Abschliessend danke ich ganz herzlich denen, die durch ihre Fürsorge und Unterstützung das Studium der Physik und diese Promotion überhaupt erst möglich gemacht haben, meinen Eltern Hildburg und Günter Janßen. Dieser Dank soll auch meine Schwester Sonja Schüttlöffel mit einbeziehen, die nicht nur zur Promotionszeit eine feste Säule meines Lebens war. 



\section{Lebenslauf}

\section{Persönliches}

Name

Katja Janßen

Geburtsort

Bremerhaven

Geburtsdatum

12. Dezember 1973

Familienstand

Staatsangehörigkeit

ledig

deutsch

\section{Schulbildung}

August 1980 - Juli 1984

August 1984 - Juli 1986

August 1996 - Juli 1990

August 1990 - Juli 1993

Abschluss

Grundschule

Alfred-Delp-Schule, Bremerhaven

Orientierungsstufe

Edith-Stein-Schule, Bremerhaven

Gymnasium

Pestalozzischule II, Bremerhaven

Oberstufe

Bürgermeister-Schmidt-Schule, Bremerhaven

Abitur

\section{Studium}

September 1993 - Dezember 1999

Abschluss

Mathematik und Physik für Lehramt an Gymnasien Georg-August-Universität, Göttingen

1. Staatsexamen

\section{Promotion}

Januar 2000 - Juli 2003

Promotion an der Universitäts-Sternwarte, Göttingen

April 2000 - März 2001

Stipendium des Graduiertenkollegs

für Strömungsinstabilitäten und Turbulenz

April 2001 - März 2003 